\title{
Reliability \\ analysis for \\ structural \\ design
}

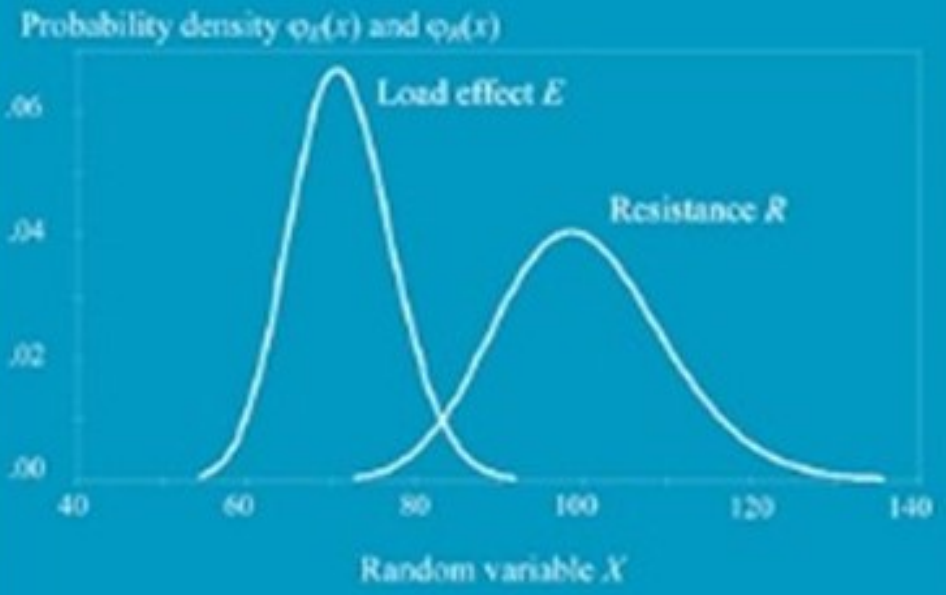




\section{Reliability \\ analysis for \\ structural \\ design}

Milan Holický 


\section{Reliability analysis for structural design}

Published by SUN MeDIA Stellenbosch

Ryneveld Street, Stellenbosch, 7600

www.africansunmedia.co.za

www.sun-e-shop.co.za

All rights reserved.

Copyright (C) 2009 Milan Holický

No part of this book may be reproduced or transmitted in any form or by any electronic, photographic or mechanical means, including photocopying and recording on record, tape or laser disk, on microfilm, via the Internet, by e-mail, or by any other information storage and retrieval system, without prior written permission from the publisher.

First edition 2009

ISBN: 978-1-920338-11-4

e-ISBN: 978-1-920689-34-6

DOI: $10.18820 / 9781920689346$

Cover design by Sonja Badenhorst

Typesetting: Author

Packaging, printing and binding: SUN MeDIA Stellenbosch

SUN PReSS is an imprint of SUN MeDIA Stellenbosch. Academic, professional and reference works are published under this imprint in print and electronic format. This publication may be ordered directly from www.sun-e-shop.co.za. 


\section{CONTENTS}

FOREWORD

1 BASIC CONCEPTS

$1.1 \quad$ Uncertainties ............................................................................ 5

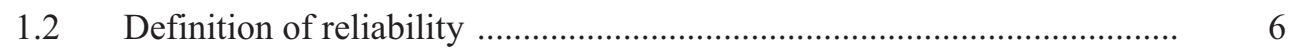

1.3 Historical development of design methods ............................................ 7

1.4 Design working life and design situation .................................................. 9

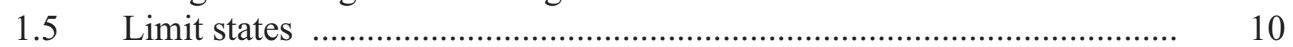

$1.6 \quad$ Ultimate limit states .................................................................... 12

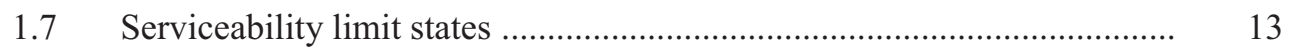

$1.8 \quad$ Reliability differentiation .................................................................... 15

Appendix A: Reinforced concrete slab - various design concepts .......... 17

2 PROBABILITY THEORY IN STRUCTURAL RELIABILITY

2.1 Experiment, random event, sample space .............................................. 21

2.2 Relations between random events ....................................................... 24

$2.3 \quad$ Definition of Probability .................................................................... 26

2.4 Basic rules for the computation of probabilities ..................................... 28

2.5 Conditional probability ..................................................................... 29

$2.6 \quad$ Bayes' theorem ….............................................................................. 31

$2.7 \quad$ Updating of probabilities ................................................................... 32

3 SELECTED MODELS OF RANDOM VARIABLES

$3.1 \quad$ Random variable ........................................................................ 35

3.2 Sample characteristics ............................................................. 38

$3.3 \quad$ Normal distribution ..................................................................... 40

3.4 Log-normal distribution ................................................................... 41

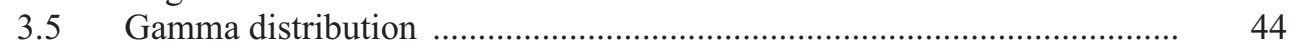

3.6 Beta distribution ........................................................................ 45

3.7 Gumbel and other distributions of extreme values .............................. 48

$3.8 \quad$ Multivariate random variables .............................................................. 51

3.9 Combination of two random samples .................................................. 53

3.10 Functions of random variables ......................................................... 56

3.11 Updating of probability distributions .................................................. 56

4 FRACTILE OF RANDOM VARIABLES

4.1 Fractile of theoretical models ................................................................ 59

4.2 Fractile estimation from samples - coverage method ............................ 63

4.3 Fractile estimation from samples - prediction method ............................... 63

4.4 Comparison of the coverage and prediction methods ............................ 64

4.5 Fractile estimation from samples - Bayes' method ................................ 68

4.6 Estimation of fractiles according to Eurocodes .................................... $\quad 70$

4.7 Fractile estimation using updated distribution ....................................... 71 


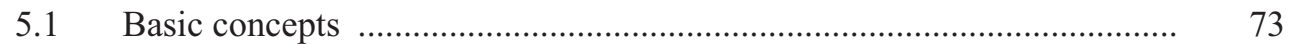

5.2 Fundamental cases of one random variable ............................................ 74

5.3 Two random variables having normal distribution ................................. 77

5.4 Two random variables having general distribution ................................. $\quad 79$

5.5 Design point in Eurocodes ....................................................................... 81

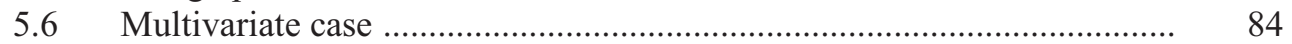

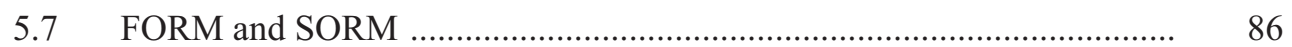

$5.8 \quad$ Simulation methods .......................................................................... 89

5.9 Target reliability level f...................................................................... 91

5.10 Probabilistic optimisation ...................................................................... 92

6 TIME-VARIANT RELIABILITY

6.1 General considerations ........................................................................ 95

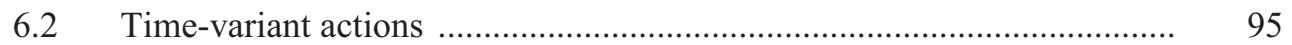

6.3 Rectangular wave processes ................................................................ 97

6.4 Rectangular wave processes with intermittencies ................................. 98

6.5 Combination of actions, Turkstra's rule ................................................ 99

6.6 Combination value of variable actions ................................................. 101

6.7 Frequent and quasi-permanent values ................................................. 103

6.8 Deterioration of structural members ..................................................... 105

7 RELIABILITY UNDER VARIABLE LOADS WITH INTERMITTENCIES

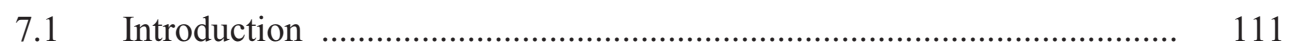

7.2 Model structure ................................................................... 111

7.3 Limit state function .............................................................. 114

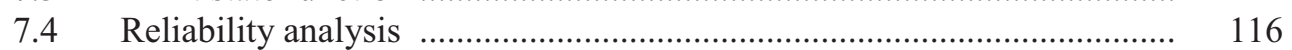

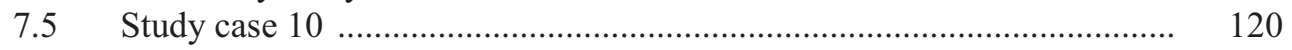

7.6 Concluding remarks ................................................................. 122

8 RELIABILITY BASIS OF THE PARTIAL FACTOR METHOD

$8.1 \quad$ Introduction ........................................................................... 123

8.2 The design value method ........................................................... 123

8.3 Reliability verification in Eurocodes ................................................ 124

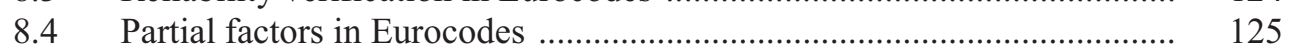

8.5 Partial factors for material property .................................................... 126

8.6 Partial factors for permanent load ...................................................... 128

8.7 Partial factors for variable load .................................................... 129

8.8 Partial factors for wind action ............................................................. 131

8.9 Concluding remarks .................................................................... 133

9 SYSTEM RELIABILITY

9.1 General ............................................................................... 135

9.2 Parallel system …............................................................ 137

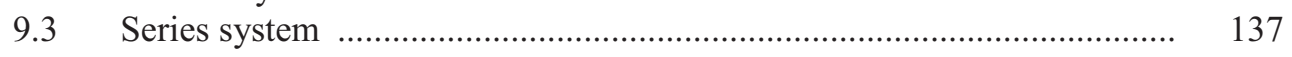

Appendix: SYSREL input file ......................................................... 140 
$10.1 \quad$ General procedure .......................................................................... 141

10.2 Hazard identification ........................................................................... 143

10.3 Definition and modelling of relevant scenarios ...................................... 143

10.4 Estimation of probabilities ..................................................................... 143

10.5 Estimation of consequences ............................................................... 144

10.6 Estimation of risk .......................................................................... 144

$10.7 \quad$ Logic trees ................................................................................ 145

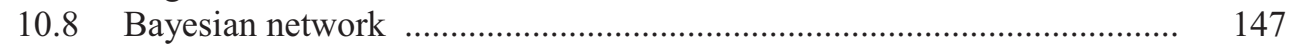

10.9 Decision-making .............................................................................. 149

10.10 Concluding remarks ........................................................................... 150

Appendix: Terminology of risk assessment ......................................... 151

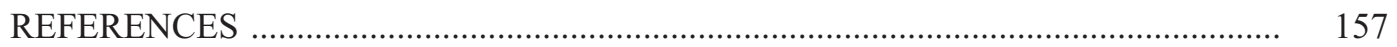

ANNEXES

ANNEX 1 - Probabilistic models of basic variables .................................................... 159

ANNEX 2 - Fractile of a random variable ............................................................... 160

ANNEX 3 - Statistical parameters of functions of random variables ........................... 161

ANNEX 4 - Conventional probabilistic models of basic variables .............................. 162

ANNEX 5 - Partial factor method and probabilistic design ........................................ 175

ANNEX 6 - List of selected software tools supplementing the main text ....................... 184

ANNEX 7 - System of Matlab functions for probabilistic structural design .................. 187

ANNEX 8 - Excel sheet FORM7 and RORMRCB ..................................................... 191

ANNEX 9 - Mathcad sheet FORM7 …........................................................................ 194

ANNEX 10 - Matlab sheet FORM7 …....................................................................... 196

ABOUT THE AUTHOR …................................................................................ 199 


\section{FOREWORD}

The theory of structural reliability becomes a powerful tool when used for the development of new standards or, alternatively, for the direct verification of both new and existing structures. Recently revised national and international standards for structural design are systematically based on probabilistic concepts, mathematical statistics and the theory of structural reliability. This approach has also been used by the European Committee for Standardization (CEN) in developing the new European standards for structural design, called Eurocodes [1], and by the International Standard Organisation (ISO) in developing recent International Standards [2, 3]. While the ISO documents are of a general nature, the Eurocodes provide more specific operational provisions based on the partial factor method. The submitted textbook explains the basis of reliability theory and attempts to clarify the links between the reliability principles and the partial factor method accepted in the newly developed standards.

The Eurocodes and the International Standards (ISO) are important basic documents for subsequent international standardisation and revision of national codes of practice. It is foreseen that in the near future a number of countries across the world will design civil structures using significantly unified methodical principles and harmonised operational provisions. The reliability verification of buildings and other civil engineering works may then differ only by numerical values of some reliability elements, such as the characteristic values of climatic actions and partial factors. It is well recognised that this remarkable achievement would not be possible without the recent progress made with the reliability theory and the development of relevant software products, which is why the theory of structural reliability is becoming a progressively more important scientific branch that is thoroughly investigated and applied by many specialists.

The development of both the European standards and the ISO documents is, however, a long process (dating back to 1970), which was accelerated in 1989 when CEN established the Technical Committee 250 (TC 250), now liable for developing the Eurocodes. The TC 250 is directly responsible for the fundamental standard "Basis of structural design" denoted by the alphanumeric denomination EN 1990 [1]. The Committee has nine subcommittees ( $\mathrm{SC} 1$ to $\mathrm{SC}$ ) that are responsible for an additional nine Eurocodes denoted as EN 1991 to EN 1999, each having several specific parts. At present, individual parts of the Eurocodes are being transformed from the previously published prestandards, prefixed ENV, to operational Eurocodes with the prefix EN.

It should be noted that the work of TC 250 is based on the principles provided in the Construction Products Directives 89/106/EEC (the European Economic Community) from 1989 and in the subsequent Interpretative Documents, ID 1 "Mechanical Resistance and Stability", ID 2 "Safety in case of fire", and partly on other Interpretative Documents (published in the Official Journal of the European Communities 94/C 62/01). It is a requirement that the structural reliability be guaranteed during the whole economically reasonable working life. In particular, the construction works must be designed and built in such a way that the loading liable to action during its construction and usage do not cause:

a) collapse of the whole or a part of the work;

b) major deformations to an inadmissible degree;

c) damage to other parts of the works, equipment or installed devices; and

d) damage by an event to an extent disproportionate to the original cause.

Similar fundamental concepts are provided in the International Standards developed by ISO $[2,6]$, as well.

The verification of the structural reliability is based on the concept of design situations and relevant limit states in conjunction with the partial factor method [1,2]. The design 
situations should encompass all conditions that can be reasonably expected to occur during the execution and use of the structure. In general, four types of design situations are recognised:

$$
\begin{aligned}
& \text { - } \quad \text { persistent situations, which refers to the conditions of normal use; } \\
& \text { - } \quad \text { transient situations, which refers to temporary conditions; } \\
& \text { - } \quad \text { accidental situations, which refers to exceptional conditions; and } \\
& \text { - } \quad \text { seismic situations, which refers to seismic events. }
\end{aligned}
$$

The limit states denote particular circumstances beyond which the structural performance requirements are no longer satisfied. A distinction is made between ultimate limit states and serviceability limit states. The ultimate limit states are those associated with the various forms of structural failure or states close to structural failure. In particular the ultimate limit states may require consideration of

$$
\begin{array}{ll}
\text { - } & \text { loss of equilibrium of the structure considered as a rigid body; and } \\
\text { - } & \text { excessive deformation or settlement, rupture, or loss of stability. }
\end{array}
$$

The serviceability limit states are those associated with the criteria for the structure related to its use or function. In particular, the serviceability limit states may require consideration of:

$$
\begin{array}{ll}
- & \text { deformation or deflection; } \\
- & \text { vibration which limits the structural use; and } \\
\text { - } & \text { detrimental cracking. }
\end{array}
$$

The Interpretative Document ID 1 states that the design rules may be based on the partial safety factor format and a desired reliability level may be established by using probabilistic reliability methods. To ensure reliability the following can be used:
a) representative values of actions;
b) values of partial safety factors;
c) requirements on ultimate limit states and serviceability limit states;
d) durability requirements;
e) measures that exclude damage disproportionate to the original cause;
f) accurate mechanical models;
g) consistent application of constructional rules; and
h) various procedures of quality provision.

Individual states may modify some of the above-listed measures in respect of the local territorial conditions. However, to implement and apply the newly developed standards effectively, a basic knowledge of the theory of probability, mathematical statistics and the theory of reliability needs to be used by a wide technical community including practising engineers. The new concepts and techniques including unusual terms (for example characteristic value, representative value, probability, fractile, reliability index, safety and serviceability) become frequently used key words that might not be always well understood. Obviously, without the correct interpretation of these terms by all potential users (designers, practising engineers and technicians, representatives of public authorities) the new design concepts could hardly be effectively applied.

Moreover, the newly developed European and International Standards allow the design of structures directly by probabilistic methods of structural reliability as an alternative procedure to the partial factor method [1,2]. The direct use of reliability methods is becoming an important tool for the design and assessment of an increasing number of civil engineering works. It refers primarily to complex and large technical systems including bridges, tunnels 
and power stations. Over the next decade the direct use of the reliability theory will very likely be on the increase. Furthermore, structural reliability forms the basis of contemporary systems of quality control and their operational techniques $[4,5,6]$. Obviously, these new concepts in the design of new and existing structures require adequate tools and techniques to be provided in the theory of structural reliability.

At present, however, only a limited number of specialists are acquainted with the theory of structural reliability. It would appear that a need exists for a basic and user-friendly textbook such as this one that demonstrates the practical significance of the reliability theory. The main purpose of this textbook is to provide an introductory text on the reliability analysis applied to structural design. It is aimed at a broad spectrum of technicians that includes practising engineers, authorities responsible for regulation and quality control, and university students.

The principle objective is to clarify the basic concepts of the theory of probability, mathematical statistics and the general theory of structural reliability which are applied in the new international and European documents for verifications of structural reliability. Emphasis is given to practical applications in the development of the partial factor method and the direct verification of structural reliability. The examples and guidance given in the book also recognise the role of computers and software products now available to the professions.

Basic terms and concepts concerning uncertainties and the reliability of civil structures are introduced in chapter 1 . Chapter 2 deals with the necessary fundamental knowledge of the theory of probability. Selected theoretical models of continuous random variables are summarised in chapter 3. One of the keywords of the new documents, used in the assessment of characteristic, representative and design values, is the fractile; this notion is therefore described in detail in chapter 4 . The basic concepts and procedures of the theory of reliability, which are accepted as the basic principles for the development of the partial factor method in the new ISO and CEN documents, are covered in chapter 5. The following chapter 6 is devoted to time-dependent phenomena, which are becoming more and more important aspects of structural reliability. Chapter 7 describes applications of the reliability analysis under time variant loads with intermittencies. Chapter 8 provides reliability backgrounds and an operational technique for the specification of partial factors accepted in the new documents of ISO and CEN. System reliability is shortly described in chapter 9. The last chapter (chapter 10) describes the basic concepts and procedures of risk assessment. The main text is supplemented by 10 annexes providing additional techniques and useful practical tools facilitating the effective use of the reliability analysis in structural design.

The textbook is written in simple language with the aim of providing a self-contained handbook or reference document. On the other hand, the size of the textbook has been deliberately limited and, consequently, some procedures are introduced without the usual detailed theoretical development. In such cases a reference to specialised literature is provided. In order to make the text understandable, the theoretical procedures are often illustrated by examples, which extend the main text and propose further possible applications of the reliability theory to structural design.

The author expresses his gratitude to Dr Jana Marková and Ms Jana Pallierová from the Klokner Institute of the Czech Technical University in Prague, Professor Johan Retief and Dr Juliet Dymond from the University of Stellenbosch, and language editors of SUN MEDIA for their help in the preparation of the manuscript. 


\subsection{Uncertainties}

It is well recognised that construction works are complicated technical systems that suffer from a number of significant uncertainties at all stages of execution and use. Some uncertainties can never be eliminated absolutely and must therefore be taken into account when designing or verifying construction works. Depending on the nature of the structure, environmental conditions and applied actions some types of uncertainties may become critical. The following types of uncertainties can usually be identified:

- natural randomness of actions, material properties and geometric data;

- statistical uncertainties due to limited available data;

- uncertainties of theoretical models owing to the simplification of actual conditions;

- vagueness due to inaccurate definitions of performance requirements;

- gross errors in design, execution and operation of the structure;

- lack of knowledge of the behaviour of new materials in real conditions.

Note that the order of the listed uncertainties corresponds approximately to the decreasing amount of current knowledge and availability of theoretical tools with which to analyse them and take them into account in design.

The natural randomness and statistical uncertainties may be relatively well described by available methods of the theory of probability and mathematical statistics. In fact the Eurocode [1] and the International Standard [2] provide some guidance on how to proceed. However, lack of reliable experimental data, i.e. statistical uncertainty, particularly in the case of new materials, some actions, including environmental influences, and also some geometrical data, causes significant problems. Moreover, the available data are often inhomogeneous and obtained under different conditions (for example for material properties, imposed loads, environmental influences but also for internal dimensions of reinforced concrete cross-sections). Then, it may be difficult if not impossible to analyse such data and to use them in design.

Uncertainties of theoretical models may be to a certain extent assessed on the basis of theoretical and experimental research. Again the Standards [1,2] provide some guidance on how to proceed. The vagueness caused by inaccurate definitions (in particular of serviceability and other performance requirements) may be partially described by the theory of fuzzy sets. Up to now, however, these methods have been of little practical significance, as suitable experimental data are rarely available. Knowledge about the behaviour of new materials and structures may well gradually increase thanks to newly developed theoretical tools, and experimental research.

The lack of available theoretical tools is obvious in the instances of gross error and lack of knowledge, which are nevertheless often the decisive causes of structural failure. In order to limit the gross errors caused by human activity a quality management system, including the methods of statistical inspection and control, may be effectively applied.

Several design methods and operational techniques have been proposed and used world-wide to control the unfavourable effects of various uncertainties during a specified working life. Simultaneously, the theory of structural reliability has been developed to describe and analyse the above-mentioned uncertainties in a rational way and to take them into account in design and verification of structural performance. In fact, the development of the whole theory was initiated by observed insufficiencies and structural failures caused by 
various uncertainties. At present the theory of structural reliability is extensively used to calibrate reliability elements of newly proposed standards (partial and various reduction factors). The term "reliability" itself is, however, often used in a very broad sense and may need some clarification.

\subsection{Definition of reliability}

The term reliability is often oversimplified and used very vaguely and inaccurately. The concept of reliability is sometimes approached in an absolute (black and white) way - the structure either is or isn't reliable. In accordance with this approach the positive statement is understood as "the failure of the structure will never occur". This is, of course, an incorrect oversimplification, as failure may occur even when the structure has been correctly declared to be reliable. The interpretation of the complementary (negative) statement is usually understood more correctly: failures are admitted and the probability or frequency of their occurrence is then discussed. Thus, according to this simplified approach there should be a certain set of structural conditions determining an area of "absolute reliability" where any possibility of failure occurring is excluded. Only when exceeding this limit would the emergence of failure be admitted.

In general such a simplified interpretation is incorrect. Although it may be unpleasant, and for many perhaps unacceptable, the hypothetical area of "absolute reliability" for most structures (apart from exceptional cases) simply does not exist. On the contrary, it is necessary at the design stage to acknowledge the certain, small probability that failure may occur within the intended life span of the structure. Otherwise it would not be at all possible to design civil structures. What is then the correct interpretation of the keyword "reliability" and what meaning does the generally used statement "the structure is safe" have?

In structural design a number of similar definitions of the term reliability, or their interpretations, are used in literature and in national and international documents. ISO 2394 [2] provides a definition of reliability which is similar to the approach of other national and international standards: reliability is the ability of a structure to comply with given requirements under specified conditions during the intended life for which it was designed.

In Eurocode [1] no definition is offered and it is noted that reliability covers only the load-bearing capacity, serviceability as well as the durability of a structure. In the Fundamental requirements it is then stated that "a structure shall be designed and executed in such a way that it will, during its intended life with appropriate degrees of reliability and in an economic way:

- remain fit for the use for which it is required; and

- sustain all actions and influences likely to occur during execution and use."

Generally, a different level of reliability for load-bearing capacity and for serviceability may be accepted. In document [1] the probability of failure $p_{\mathrm{f}}$ and the reliability index $\beta$ are related to failure consequences.

Note that the above definition of reliability includes four important elements:

- given (performance) requirements - the definition of structural failure;

- time period - the assessment of the required service-life $T$;

- reliability level - the assessment of the probability of failure $p_{\mathrm{f}}$; and

- conditions of use - limiting input uncertainties. 
An accurate determination of performance requirements and thus an accurate specification of the term failure is of primary importance. In many cases, mainly when considering the requirements for the stability and collapse of a structure, the specification of this term is not very complicated, although in many other cases, in particular when dealing with various requirements of occupants' comfort, and the appearance and characteristics of the environment, the appropriate definitions of failure are dependent on vagueness and inaccuracies. The translation of these occupants' requirements into appropriate technical quantities and precise criteria can be very difficult and often leads to very vague conditions. In the following the term failure is used in a very general sense denoting simply any undesirable state of a structure (for example collapse or excessive deformation) which has been unambiguously given by structural conditions.

\subsection{Historical development of design methods}

During their developmental stages those design methods that took into account recognised uncertainties and ensured structural reliability have been closely linked to the available empirical, experimental, as well as theoretical knowledge of mechanics and the theory of probability. The development of various empirical methods for structural design gradually stabilised in the twentieth century according to three generally used methods which are, with various modifications, being applied in standards for structural design to this day. In the context of efforts to simplify the computational procedures some of these methods are sometimes modified or rehabilitated. That is why it is useful to briefly mention these three basic design methods and to indicate the explicit measures that might affect the probability factors of failure and structural reliability.

The first universally-accepted design method for civil structures is the method of permissible stresses. It is based on the condition

$$
\sigma_{\max }<\sigma_{\text {per }} \text {, where } \sigma_{\text {per }}=\sigma_{\text {crit }} / k
$$

where the coefficient $k$ is assessed with regard to uncertainties in the determination of local load effect $\sigma_{\max }$ and of resistance $\sigma_{\text {per }}$ and therefore may ensure with an appropriate level of security the reliability of the structure. The main insufficiency of this method is perhaps the local verification of reliability (in the elastic range) and the impossibility to consider separately the uncertainties of basic quantities and the uncertainties of computational models for the assessment of action effects and structural resistance. In this method, the probability of failure is controlled by one quantity only, the coefficient $k$.

The second widely-accepted method of structural design is the method of global safety factor. It is based on the condition

$$
s=X_{\text {resist }} / X_{\text {act }}>s_{0}
$$

according to which the calculated safety factor $s$ must be greater than its specified value $s_{0}$. It is a method which attempts mainly to give a truer picture of the behaviour of elements and their cross-sections, in particular through the aggregate quantities of structural resistance $X_{\text {resist }}$ and action effect $X_{\text {act }}$. As in the case of the permissible stresses method the main insufficiency of this method remains the impossibility to consider the uncertainties of particular basic quantities and theoretical models. The probability of failure can, again, be controlled by one quantity only, i.e. by the global safety factor $s$.

At present, the most advanced operational method of structural design $[1,2]$ is the partial factor format (often inaccurately denoted as the limit states method). This method is based on the condition 


$$
E_{\mathrm{d}}\left(F_{\mathrm{d}}, f_{\mathrm{d}}, a_{\mathrm{d}}, \theta_{\mathrm{d}}\right)<R_{\mathrm{d}}\left(F_{\mathrm{d}}, f_{\mathrm{d}}, a_{\mathrm{d}}, \theta_{\mathrm{d}}\right)
$$

where the action effect $E_{\mathrm{d}}$ and the structural resistance $R_{\mathrm{d}}$ are assessed according to the design values of basic quantities describing the action $F_{\mathrm{d}}$, material properties $f_{\mathrm{d}}$, dimensions $a_{\mathrm{d}}$ and model uncertainties $\theta_{\mathrm{d}}$. The design values of these quantities are determined (taking into account their uncertainties as well as the uncertainties of computational models) using their characteristic values $\left(F_{\mathrm{k}}, f_{\mathrm{k}}, a_{\mathrm{k}}, \theta_{\mathrm{k}}\right)$, partial safety factors $\gamma$, combination factors $\psi$ and other measures of reliability $[1,2,6]$. Thus, a whole system of various partial factors and other reliability elements may be used to control the probability of structural failure.

Obviously, the greatest possibility of harmonising structural reliability of different structures made of different materials is offered by the partial factor method. However, in any of the listed methods the probability of failure is not applied directly. Among standards for structural design the recent document ISO [2] was the first one to include probabilistic methods.

Probabilistic design methods [2] are based on the condition that the probability of failure $p_{\mathrm{f}}$ does not exceed a specified target value $p_{\mathrm{t}}$ during the service life of a structure $T$

$$
p_{\mathrm{f}} \leq p_{\mathrm{t}}
$$

It is usually possible to assess the probability of failure $p_{\mathrm{f}}$ using a computational structural model, defined through basic quantities $\boldsymbol{X}\left[X_{1}, X_{2}, \ldots, X_{\mathrm{n}}\right]$ for actions, mechanical properties and geometrical data. The limit state of a structure is defined by the limit state function (the performance function) $g(\boldsymbol{X})$ for which, according to the definition, in case of a favourable (safe) state of the structure the limit state function is positive; it holds that

$$
\mathrm{g}(\boldsymbol{X}) \geq 0
$$

and the unfavourable state (failure) of the structure occurs when the limit state function is negative, i.e. when $\mathrm{g}(\boldsymbol{X})<0$ (a more detailed explanation is given in chapter 5).

Basic quantities are generally time-dependent (stochastic) functions. Even so, in most cases it is sufficient to describe them by time-independent models having, however, their characteristics deduced for extreme (maximal or minimal) values of the appropriate quantity (action or resistance) during the specified design life $T$.

For most ultimate limit states and serviceability limit states the probability of failure can be expressed by the equation

$$
p_{\mathrm{f}}=\mathrm{P}\{\mathrm{g}(\boldsymbol{X})<0\}
$$

More complicated procedures need to be used when some of the quantities are timedependent. Some details concerning theoretical models for time-dependent quantities (mainly actions) and their use for structural reliability analysis are given in chapter 6. However, in many cases the problem may be transformed to a time-independent one, for example by considering in equation (1.6) a minimum of the function $\mathrm{g}(\boldsymbol{X})$ over the time period $T$.

The assessment of various reliability measures (characteristic values, partial and combination factors) in the new structural design standards [1,2] is partially based on probabilistic considerations but to a great extent they are based on historical and empirical experience. Moreover, the choice of these reliability measures is, in Eurocode 1 [1], affected by the intention to simplify the calculation in practical design. This intention, however, leads sometimes to oversimplification and, consequently, to the increase of material consumption.

In this connection there is an obvious question how to harmonise the new design codes for various structures on the basis of general principles of the theory of reliability if the current intention to simplify computational procedures suppresses the advantages of the partial factors method. This question is hard to answer [41]. Material consumption is only one criterion of evaluation and need not be the most important. A broad discussion by experts has 
shown clearly that in order to make a critical analysis of the new codes there are other criteria that should also be taken into consideration. Besides the material consumption, they include laboriousness of design and construction, maintenance and repairs, service life, insurance, material recycling, possibility of changing the occupancy and others. Analysis of these criteria will surely be the subject of many investigations and optimisation studies.

Today, one thing already seems to be clear. A further improvement of current methods will be based on calibration procedures, optimisation methods and other rational approaches including the use of the methods of the theory of probability, mathematical statistics and the theory of reliability. The keyword of all these procedures is the probability of failure $p_{\mathrm{f}}$, and although it has a limited informative ability, it remains the most general, common measure of structural reliability. Methods of the theory of reliability then provide the most important tool for the gradual improvement and harmonisation of design for various structures from different materials. The theory of structural reliability also makes it possible to extend the general methodology for new structures and materials.

Annex A to this chapter shows a simple example of reinforced concrete slab designed in accordance with the above-mentioned techniques. This example will also be used in the following chapters to illustrate the application of more advanced probabilistic approaches.

\subsection{Design working life and design situation}

The design working life denotes the period for which a structure or part thereof is to be used for its intended purpose with anticipated maintenance but without major repair being necessary. Table 1.1 taken from EN 1990 [1] gives categories together with the indicated design working life for a number of common types of construction works.

Table 1.1. Indicative design working life

\begin{tabular}{||c|c|l||}
\hline $\begin{array}{l}\text { Design } \\
\text { Working } \\
\text { Life } \\
\text { Category }\end{array}$ & $\begin{array}{l}\text { Notional } \\
\text { Design } \\
\text { Working } \\
\text { Life (years) }\end{array}$ & \multicolumn{1}{||}{ Examples } \\
\hline 1 & 10 & Temporary structures (e.g. scaffolding) \\
\hline 2 & $10-25$ & $\begin{array}{l}\text { Replaceable structural parts, e.g. gantry girders, bearings (see } \\
\text { appropriate standards) }\end{array}$ \\
\hline 3 & $15-30$ & $\begin{array}{l}\text { Agricultural and similar structures (e.g. buildings for animals } \\
\text { where people do not normally enter) }\end{array}$ \\
\hline 4 & 50 & $\begin{array}{l}\text { Building structures and other common structures (e.g. } \\
\text { hospitals, schools etc) }\end{array}$ \\
\hline 5 & 100 & $\begin{array}{l}\text { Monumental building structures, bridges and other civil } \\
\text { engineering structures (e.g. churches) }\end{array}$ \\
\hline
\end{tabular}

The present state of knowledge is insufficient to enable precise prediction of the life of a structure. The behaviour of materials and structures over extended periods of time can only be estimated. The likely period of maintenance of the structure or time of replacements of the various components of a structure can, nevertheless, be determined. 
However, the notion of a design working life is useful for:

- the selection of design actions (for example imposed, wind, earthquake etc.) and the consideration of material property deterioration (for example fatigue, creep) in reliability verification;

- the comparison of different design solutions and choice of materials, each of which will give a different balance between the initial cost and cost over an agreed period life cycle costing will need to be undertaken to evaluate the relative economics of the various solutions;

- the evolving of management procedures and strategies for systematic maintenance and renovation of structures.

In design the variation of actions, environmental influences and structural properties, which occur throughout the design working life of a structure, should be considered by selecting distinct situations representing a certain time interval with associated hazards

Four design situations are classified in EN 1990 [1] as follows:

(a) Persistent situations refer to conditions of normal use. These are generally related to the design working life of the structure. Normal use can include possible extreme loading conditions from wind, snow, imposed loads, etc.

(b) Transient situations refer to temporary conditions of the structure, in terms of its use or its exposure, for example during construction or repair. This implies the use of a time period much shorter than the design working life; one year may be adopted in most cases.

(c) Accidental situations refer to exceptional conditions of the structure or of its exposure, for example due to fire, explosion, impact, local failure. This implies the use of a relatively short period, but not for those situations where a local failure may remain undetected.

(d) Seismic situations refer to exceptional conditions applicable to the structure when subjected to seismic events.

These design situations should be selected so as to encompass all conditions that are reasonably foreseeable as occurring during the execution and use of the structure. For example a structure after an accidental design situation due to actions such as fire or impact may need a repair (short time period of about one year), for which the transient design situation should be considered. In general a lower reliability level and lower partial factors than those used for persistent design situation might be applicable for this period of time. However, it should be mentioned that the repair should be designed taking into consideration of all the other foreseeable design situations.

\subsection{Limit states}

Traditionally, according to the fundamental concept of limit states it is considered that the states of any structure may be classified as either satisfactory (safe, serviceable) or unsatisfactory (failed, unserviceable). Distinct conditions separating satisfactory and unsatisfactory states of a structure are called limit states. Thus, the limit states are those beyond which the structure no longer satisfies the design criteria. Each limit state is therefore associated with a certain performance requirement imposed on a structure. Often, however, these requirements are not formulated sufficiently clearly so as to allow for precise (sharp) definition of appropriate limit states.

Generally, it may be difficult to express the performance requirements qualitatively and to define the limit states unambiguously (particularly the ultimate limit states of structures made of ductile materials, and also the serviceability limit states, typically those affecting user 
comfort). In these cases, only a suitable approximation is available (for example the conventional yield point of metals, or a limiting value for vertical deflection). The principles of this are indicated in Figure 1.1 and provided here as a background to the uncertainties of the limit state concept.

According to the traditional (sharp) concept of limit states described above, a given structure is assumed to be fully satisfactory up to a certain value of the load effect $E_{0}$ and beyond this value the structure is assumed to be fully unsatisfactory (see Figure 1.1 (a)). However, it may be very difficult to define precisely such a distinct value $E_{0}$, separating the desired and undesired structural conditions, and the simplification in Fig. 3.1 (a) may not be adequate. In these cases a transition region $\left\langle E_{1}, E_{2}\right\rangle$, in which a structure is gradually losing its ability to perform satisfactorily, provides a more realistic (vague) concept (see Fig. 3.1 (b)). Uncertainties in the vague concept of limit states may be taken into account only in reliability analyses using special mathematical techniques which are not covered in the present generation of Eurocodes.

(a) Sharp

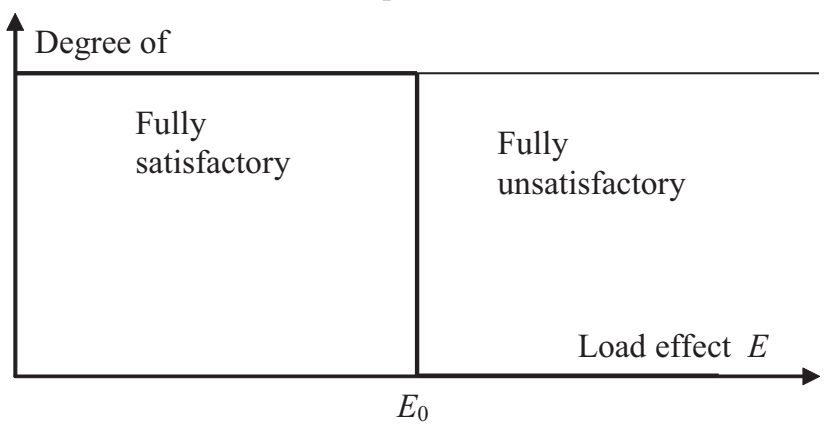

(b) Vague

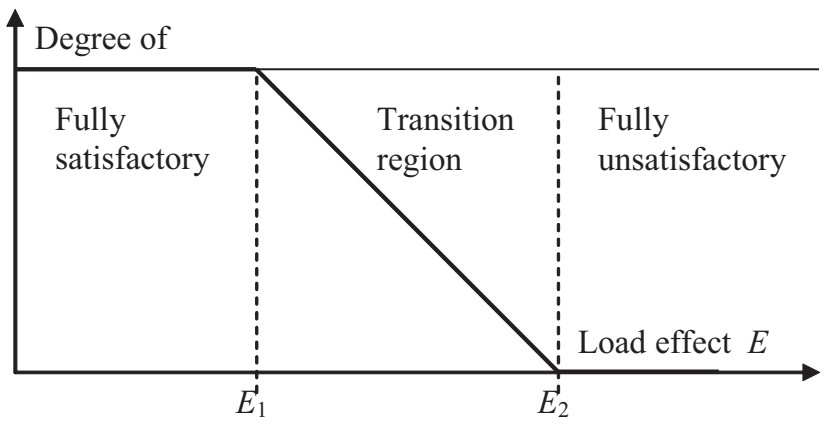

Figure 1.1. Sharp and vague definition of a limit state

In order to simplify the design procedure two fundamentally different types of limit states are generally recognised:

(a) ultimate limit states; and

(b) serviceability limit states.

Ultimate limit states are associated with collapse or other similar forms of structural failure. Serviceability limit states correspond to conditions of normal use (deflections, vibration, cracks, etc.). In general the design should include both safety and serviceability, including durability in both cases. The nature of ultimate limit states is essentially different from the nature of serviceability limit states and should be taken into account in reliability verification. There are two main reasons for this distinction: 
(a) While the infringement of ultimate limit states leads almost always to the overall loss of structural reliability and to the removal or fundamental repair of the structure, the infringement of serviceability limit states does not usually lead to such fatal consequences for the structure, and the structure may normally be used after the removal of those actions which caused the infringement.

(b) While the criteria of ultimate limit states involve parameters of the structure and appropriate actions only, the criteria of serviceability limit states are also dependent on the requirements of the client and users (sometimes very subjective), and on the characteristics of the installed equipment or non-structural elements.

The differences between the ultimate limit states and serviceability limit states result in a separate formulation of reliability conditions, and in dissimilar reliability levels assumed in the verification of both types of limit states. However, verification of one of the two limit states may be omitted if sufficient information is available to ensure that the requirements of the one limit state are met by the other limit state. For example in the case of reinforced concrete beams designed for an ultimate limit state it is allowed to omit verification of deflection provided that span/effective depth ratio is less than 18 for highly stressed concrete or less than 25 in case of lightly stressed concrete.

Variation of actions, environmental influences and structural properties, which occur throughout the life of the structure, should be considered in design by selecting distinct situations (persistent, transient, accidental and seismic) representing a certain time interval with associated hazards. The ultimate and serviceability limit states should be considered in all these design situations, which should be selected so as to encompass all conditions which are reasonably foreseeable or occurring during the execution and use of the structure. Within each load case, a number of realistic arrangements should be assumed to establish the envelope of action effects, and which should be considered in the design.

If the limit states considered in design are dependent on time-variant effects (described by action and/or resistance variables), the reliability verification of a structure should be related to the design working life. It should be mentioned that most time-dependent effects (for example fatigue) have a cumulative character that should be taken into account.

\subsection{Ultimate limit states}

The ultimate limit states are associated with collapse and other similar forms of structural failure and directly concern the safety of the structure and the safety of people. However, in some cases the ultimate limit states may concern also the protection of the contents, for example of some chemicals or nuclear or other waste materials.

In almost all cases which concern the ultimate limit states the first passage of the limit state is equivalent to failure. In some cases, for example when excessive deformations are decisive, states prior to structural collapse can, for simplicity, be considered in place of the collapse itself and treated as ultimate limit states. These important circumstances should be taken into account when specifying the reliability parameters of structural design and quality assurance. For example in the case of foundations of rotating machinery used in power plants excessive deformation is decisive and entirely governs the design.

The following list provides the most typical ultimate limit states that may require consideration in the design:

(a) loss of equilibrium of the structure or any part of it, considered as a rigid body;

(b) failure of the structure or part of it due to rupture, fatigue or excessive deformation;

(c) instability of the structure or one of its parts;

(d) transformation of the structure or part of it into a mechanism; and 
(e) sudden change of the structural system to a new system (for example snap through).

Time-dependent structural properties, such as fatigue and other time-dependent deterioration mechanisms reduce the strength of a structure and can initiate one of the abovementioned ultimate limit states. In this respect it is useful to distinguish two types of structures: damage tolerant (i.e. robust) and damage intolerant (sensitive to minor disturbance or construction imperfections). Effects of various deteriorating mechanisms on the ultimate limit states should then be taken into account according to the type of structure.

An adequate reliability level of damage intolerant structures can also be assured by an appropriate quality control programme. In the case of damage tolerant structures, fatigue damage may be regarded as a serviceability limit state. Note that different sets of partial factors may be associated with the various ultimate limit states.

\subsection{Serviceability limit states}

The serviceability limit states are associated with conditions of normal use. In particular they concern the functioning of the structure or structural members, the comfort of people and appearance of the construction works.

Taking into account the time-dependency of load effects it is useful to distinguish two types of serviceability limit states which are illustrated in Figure 1.2.

(a) Irreversible serviceability limit states (see Figure 1.2(a)), which are those limit states that remain permanently exceeded even when the actions which caused the infringement are removed (for example a permanent local damage, permanent unacceptable deformations);

(b) Reversible serviceability limit states (see Figure 1.2(b)), which are those limit states that will not be exceeded when the actions that caused the infringement are removed (for example cracks of prestressed components, temporary deflections, excessive vibration). 
(a) Irreversible limit state

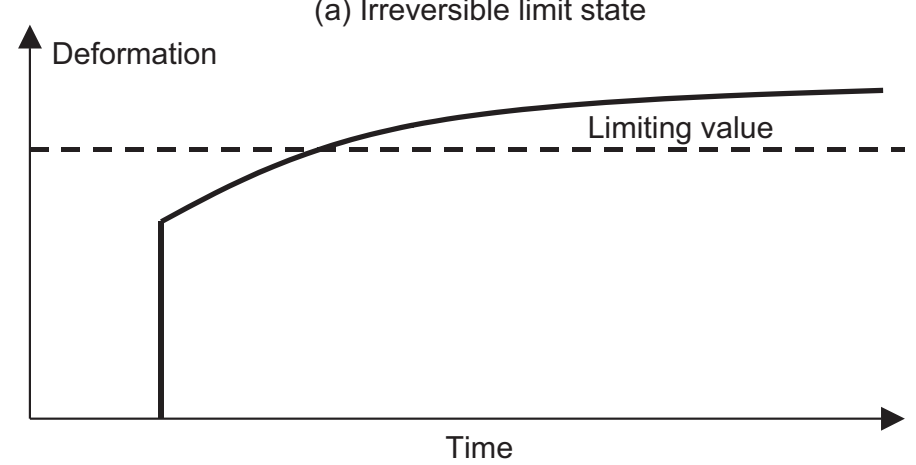

(b) Reversible limit state

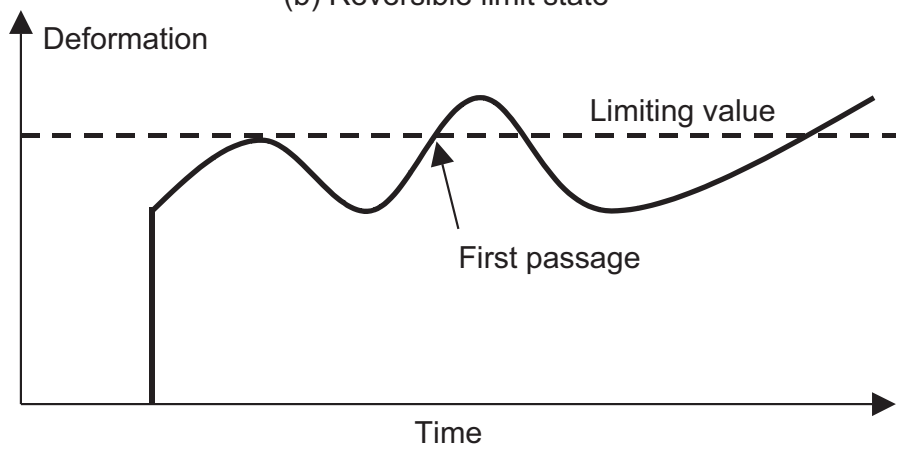

Figure 1.2. Irreversible and reversible limit states

For irreversible limit states the design criteria are similar to those of ultimate limit states. The first passage of the limit state is decisive (see Figure 3.2). This important aspect of irreversible limit states should be taken into account when determining the serviceability requirements in the contract or design documentation. For reversible limit states the first infringement (first passage) does not necessarily lead to failure and the loss of serviceability.

Various serviceability requirements can be formulated taking into account the acceptance of infringements, their frequency and their duration. Generally, three types of serviceability limit states are applicable as follows:

(a) no infringement is accepted;

(b) specified duration and frequency of infringements are accepted; and

(c) specified long-term infringement is accepted.

The correct serviceability criteria are then associated as appropriate with the characteristic, frequent and quasi-permanent values of variable actions. The following combinations of actions corresponding to the above three types of limit states are generally used in verification of serviceability limit states for different design situations:

(a) the rare (characteristic) combination if no infringement is accepted;

(b) the frequent combination if the specified time period and frequency of infringements are accepted; and

(c) the quasi permanent combination if the specified long-term infringement is accepted.

The list of serviceability limit states affecting the appearance or effective use of the structure, which may require consideration in the design, may be summarised as follows:

(a) excessive deformation, displacement, sag and inclination which can affect, for example, the appearance of the structure, comfort of users, functioning of the structure and can cause damages of finishes and non-structural members; 
(b) excessive vibration (acceleration, amplitude, frequency) which can, for example, cause discomfort to people and limit the functioning of the structure; and

(c) damage that is likely to adversely affect the appearance (local damage and cracking), durability or functioning of the structure.

Depending on the type of structure additional requirements related to serviceability limit states may be found in material-oriented codes. For example in the case of concrete structures the ultimate limit states may be induced by structural deformation.

\subsection{Reliability differentiation}

For the purpose of reliability differentiation EN 1990 [1] establishes reliability classes RC (also called consequence classes (CCs)). Three classes are defined in accordance with consequences of failure or malfunction of the structure as follows:

Reliability Class, RC3

High consequence for loss of human life, or economic, social or environmental consequences very great

Reliability Class, RC2

Medium consequence for loss of human life, economic, social or environmental consequences considerable

Reliability Class, RC1

Low consequences for loss of human life, and economic, social or environmental consequences small or negligible

Table 1.2 gives the recommended minimum values for the reliability index $\beta$ associated with reliability classes (RC) for ultimate limit states only, fatigue and serviceability limit states as indicated in EN 1990 [1].

Table 1.2. Reliability classes and recommended minimum values for reliability index $\beta$

\begin{tabular}{|c|c|c|c|c|c|c|}
\hline $\begin{array}{c}\text { Reliability } \\
\text { Class }\end{array}$ & \multicolumn{6}{|c|}{ Minimum values for $\beta$} \\
\hline & \multicolumn{2}{|c|}{ Ultimate limit states } & \multicolumn{2}{|c|}{ Fatigue } & \multicolumn{2}{|c|}{ Serviceability } \\
\cline { 2 - 7 } & $\begin{array}{c}1 \text {-year } \\
\text { reference } \\
\text { period }\end{array}$ & $\begin{array}{c}50 \text {-year } \\
\text { reference } \\
\text { period }\end{array}$ & $\begin{array}{c}\text { 1-year } \\
\text { reference } \\
\text { period }\end{array}$ & $\begin{array}{c}50 \text {-year } \\
\text { reference } \\
\text { period }\end{array}$ & $\begin{array}{c}\text { 1-year } \\
\text { reference } \\
\text { period }\end{array}$ & $\begin{array}{c}50 \text {-year } \\
\text { reference } \\
\text { period }\end{array}$ \\
\hline RC3 & 5,2 & 4,3 & & & & \\
\hline RC2 & 4,7 & 3,8 & & 1,5 to 3,8 & 2,9 & 1,5 \\
\hline RC1 & 4,2 & 3,3 & & & & \\
\hline
\end{tabular}

Typically, reliability class RC 2 is considered for residential and office buildings ( $\beta=$ 3,8 for a 50 -year reference period). However, it should be noted that a design using Eurocodes with the recommended values of partial factors leads to a structure with a different $\beta$ value from those indicated in Table 1.1. The probability of failure and its corresponding $\beta$ index are only notional values that do not necessarily represent actual failure rates (which depend mainly on human error). They are used as operational values for code calibration purposes and comparison of reliability levels of structures.

Note that a slightly different Table 1.3 is provided in ISO 2394 [2]. 
Table 1.3 Target $\beta$ values for life time

\begin{tabular}{|l|c|c|c|c|}
\hline \multirow{2}{*}{$\begin{array}{l}\text { Relative cost of } \\
\text { safety measures }\end{array}$} & \multicolumn{4}{|c|}{ Consequences of failure } \\
\cline { 2 - 5 } & small & some & moderate & Great \\
\hline High & 0 & 1,5 & 2,3 & 3,1 \\
\hline Moderate & 1,3 & 2,3 & 3,1 & 3,8 \\
\hline Low & 2,3 & 3,1 & 3,8 & 4,3 \\
\hline
\end{tabular}

It is further suggested to use:

A: for serviceability limit state $\beta=0$ for reversible and $\beta=1,5$ for irreversible states

$\mathrm{B}$ : for fatigue limit states $\beta=2,3$ to $\beta=3,1$ depending on the possibility of inspection

$\mathrm{C}$ : for ultimate limit states the safety classes $\beta=3,1, \beta=3,8$ and $\beta=4,3$.

Thus ISO 2394 (1) recommends similar target $\beta$ values for the ultimate limit states (the shaded part of the Table 1.3) as those indicated in EN 1990 [1].

The values given in Table 1.3 have been derived assuming log-normal or Weibul distribution for resistance, normal distribution for permanent load and Gumbel distribution for variable loads. It is emphasised that these or similar theoretical models should be used in probabilistic analysis. 


\section{Appendix A: Reinforced concrete slab - various design concepts}

\section{A.1 Introduction}

Various design methods used in history may be well illustrated by considering a simple reinforced concrete slab in an office building. Without going into technical details the example also indicates the advantages of the reliability-based partial factor method and the significance of the reliability theory in structural design compared to other design methods.

\section{A.2 A reinforced concrete slab}

A simply supported slab having the span of $6 \mathrm{~m}$ is exposed to a permanent load (the self-weight of the slab and other fixed parts of the building), which is estimated by the characteristic value (equal to the mean value) $g_{\mathrm{k}}=7 \mathrm{kN} / \mathrm{m}^{2}$. In accordance with the EN 1990 [1] the imposed load in an office area may be considered by the characteristic value $q_{\mathrm{k}}=3$ $\mathrm{kN} / \mathrm{m}^{2}$. It is, however, well known that the mean value of this imposed load is much lower, about $0,8 \mathrm{kN} / \mathrm{m}^{2}$.

Further, the concrete $\mathrm{C} 20 / 25$ having the characteristic strength $f_{\mathrm{ck}}=20 \mathrm{MPa}$ (the mean $30 \mathrm{MPa}$ ) and reinforcement bars having the characteristic strength $f_{\mathrm{yk}}=500 \mathrm{MPa}$ (the mean $560 \mathrm{MPa}$ ) are to be used. Using previous experience the height of the slab $0,2 \mathrm{~m}$ has been estimated in advance. Given the above data concerning the preliminary specifications an estimate should be done of the necessary reinforcement of the slab.

\section{A.3 Design and reliability consideration}

To simplify computational procedures it is assumed that the same assumption concerning stress distribution in the slab, shown in Figure A.1, can be accepted for all design methods. Note that the design methods based on permissible stresses assumes the linear stress distribution in the compression zone of the slab but the accepted assumption of the rectangular stress distribution (indicated in Figure A.1) is an acceptable approximation for illustration of the main features of the design methods.

The basic variables are defined as follows: $d$ denotes the effective depth, $x$ the depth of the compression zone, $b$ the width of the slab (considered as $1 \mathrm{~m}$ ), $A_{\mathrm{s}}$ the area of the reinforcement, $f_{\mathrm{c}}$ the concrete strength and $f_{\mathrm{y}}$ the reinforcement strength (yield point).

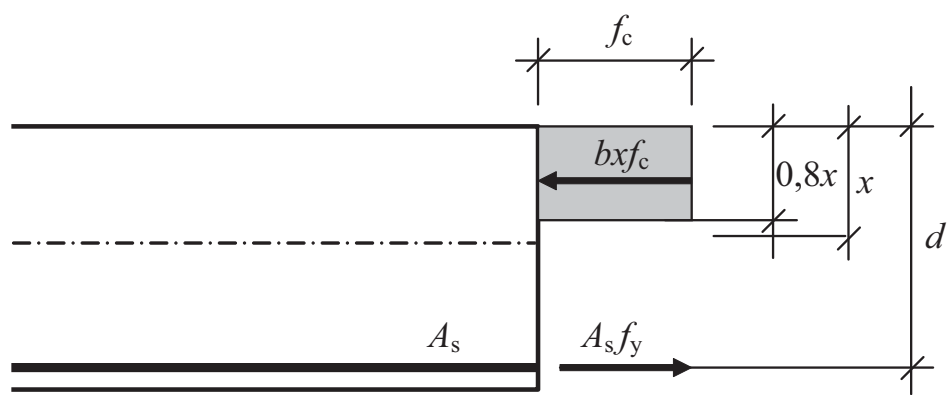

Figure A.1. Slab cross-section. 
The following equilibrium conditions follow from Figure A.1:

$$
\begin{gathered}
f_{\mathrm{c}} \times b=A_{\mathrm{s}} f_{\mathrm{y}} \\
A_{\mathrm{s}} f_{\mathrm{y}}(d-x / 2)=M
\end{gathered}
$$

Here $M=(g+q) L^{2} / 8$ denotes the bending moment due to the permanent and imposed loads $g$ and $q$. Using conditions (A.1) and (A.2) the engineer derived the following formula for the area $A_{\mathrm{s}}$ :

$$
A_{\mathrm{s}}=\frac{b\left(d-\sqrt{d^{2}-\frac{2 M}{f_{\mathrm{c}} b}}\right) f_{\mathrm{c}}}{f_{\mathrm{y}}}
$$

Up to now all the variables have been considered as deterministic quantities without taking into account uncertainties that may potentially affect their actual values. The engineer realised that the basic variables entering equation (A.3) may have a considerable scatter, but he could not remember how to take this into account. So, to get a first estimation of the area $A_{\mathrm{s}}$ he took the mean (average) values of all the basic variables involved in Figure A.1. The results of this attempt together with outcomes of various code methods are summarised in Table A.1. Note that equation (A.3) may be used generally for any design method indicated in Table A.1.

Table A.1. A reinforced concrete slab specified by different design methods for the $\operatorname{span} L=6$ $\mathrm{m}$, the height $h=0,2 \mathrm{~m}(d=0,17 \mathrm{~m})$ and the loads $g_{\mathrm{k}}=7 \mathrm{kN} / \mathrm{m}^{2}, q_{\mathrm{k}}=3 \mathrm{kN} / \mathrm{m}^{2}$ (the mean 0,8 $\left.\mathrm{kN} / \mathrm{m}^{2}\right), \mathrm{C} 25 / 20\left(f_{\mathrm{ck}}=20 \mathrm{MPa}\right.$, the mean $\left.30 \mathrm{MPa}\right), f_{\mathrm{yk}}=500 \mathrm{MPa}$ (the mean $\left.560 \mathrm{MPa}\right)$.

\begin{tabular}{lcccll}
\hline \multicolumn{1}{c}{ Design method } & $M[\mathrm{kNm}]$ & $A_{\mathrm{S}}\left[\mathrm{m}^{2}\right]$ & $\mu M_{R}[\mathrm{kNm}]$ & $\beta$ & $P_{\mathrm{f}}$ \\
\hline The mean value (absurd) & 351 & 0,000376 & 351 & 0 & 0,5 \\
The permissible stresses $(\mathrm{CP} 114)$ & 450 & 0,001161 & 1036 & 5,76 & $0,42 \times 10^{-8}$ \\
The global safety factor $(\gamma=1,9)$ & 450 & 0,001094 & 977 & 5,51 & $0,18 \times 10^{-7}$ \\
The partial factors method $(\mathrm{CEN})$ & 628 & 0,000933 & 841 & 4,82 & $0,70 \times 10^{-6}$ \\
\hline
\end{tabular}

Table A.2. Design values of the loads and material strengths.

\begin{tabular}{lllll}
\hline Basic variable & The mean & $\begin{array}{l}\text { Permissible } \\
\text { stresses }\end{array}$ & $\begin{array}{l}\text { Global } \\
\text { safety factor }\end{array}$ & $\begin{array}{l}\text { Partial } \\
\text { factors }\end{array}$ \\
\hline Permanent load $g\left[\mathrm{kN} / \mathrm{m}^{2}\right]$ & 7 & 7 & 7 & 9,45 \\
Imposed load $q\left[\mathrm{kN} / \mathrm{m}^{2}\right]$ & 0,8 & 3 & 3 & 4,5 \\
Concrete strength $f_{\mathrm{c}}[\mathrm{MPa}]$ & 30 & 5,5 & 20 & 13,3 \\
Reinforcement strength $f_{\mathrm{y}}[\mathrm{MPa}]$ & 560 & 275 & 500 & 435 \\
\hline
\end{tabular}

Table A.1 clearly indicates that "the mean values method", used by the engineer to get a first approximation, is foolish. It leads to a low reinforcement area $A_{\mathrm{S}}=0,000376 \mathrm{~m}^{2}$ (the reinforcement ratio 0.0022 only) corresponding to an unacceptably high probability of failure of 0,5 . It is well recognised that instead of the mean values other, more "safe" values of the basic variables should be used in design calculations. Without going into technical details (that are offered later in the book) Table A.2 indicates the design values of loads and material 
strengths used in accordance with the design methods indicated in Table A.1. All details necessary to determine the data given in Table A.1 and A.2 will be explained in the following text.

\section{A.4 Concluding remarks}

It follows from Table A.1 that the permissible stresses method (CP 114) seems to lead to rather conservative (and perhaps uneconomical) results, as does the method of the global safety factor. The partial safety factors method, accepted in the recent EN documents, seems to provide the most suitable design format. The reliability index $\beta=4,8$ obtained by this method is close to the target value $\beta=3,8$.

However, the most important advantage of the partial safety factors method is the possibility of taking into account the uncertainty of individual basic variables by adjusting (calibrating) relevant partial factors and other reliability elements. It is the aim of this book to explain the fundamental principles of this method and to show how appropriate design values of basic variables could be specified in order to achieve an adequate reliability level. 


\subsection{Experiment, random event, sample space}

This chapter gives a concise overview of the most important concepts and terms of the theory of probability, which are frequently used in the reliability analysis of civil engineering works and systems. The presentation of some concepts and laws is rather intuitive without rigorous mathematical proofs. More detailed explanations of all the presented concepts, theorems and rules may be found in specialised literature [11, 12, 14, 18, 19].

The most significant fundamental concepts of the theory of probability applied in structural reliability include

- experiment;

- random event; and

- sample space (space of events).

These terms are used by the classical probability theory, but are also applicable in the contemporary theory of probability based on the theory of sets.

\section{Experiment}

An experiment in the theory of probability is understood as a realization of a certain set of conditions $\pi$. In the classical approach to the theory of probability it is often assumed that the experiments can be repeated arbitrarily (e.g. tossing a dice, testing a concrete cube) and the result of each experiment can be unambiguously used to declare whether a certain event occurred or did not occur (e.g. when tossing a dice obtaining or not obtaining a predetermined number, or when a concrete cube exceeds or does not exceed a specified value).

However, in practical applications of the theory of probability the assumption of arbitrary repeatable experiments, each of which leads to an unequivocal result (even though not known beforehand), is not always realistic (e.g. in construction usually only a very limited number of experiments can be performed). Currently, when using the theory of probability more general concepts are accepted, where terms such as an experiment, event and sequence of events are related to the general theory of sets.

The concept of an experiment remains applicable in general. However, specification of the conditions $\pi$ is of utmost importance irrespective of whether the experiment can be realistically carried out. In some cases the experiment can be carried out only hypothetically. In any case the specification of the conditions $\pi$ needs to be as accurate and complete as possible. The results of any experiment and its practical interpretation should to always be related to these conditions. The comparison of experiments carried out under different conditions may lead to serious mistakes and misunderstandings. A description of the appropriate set of conditions and their verification should therefore become an indispensable part of every probabilistic analysis.

\section{Random event}

The probability theory deals with the results of experiments that are not unequivocally determined in advance by the appropriate set of conditions $\pi$, or with experiments for which a set of conditions that would lead to an unequivocal result either cannot be provided during an experiment, or is not known at all (or partly unknown). An experiment of this kind is called a random experiment. The result of a random experiment is described by events that could, but will not necessarily, occur when the conditions $\pi$ are realized. Such events are called random events and are usually denoted by a capital letter from the beginning of the alphabet, e.g. $A$ or 
$B$ (possibly with an index). An event that will necessarily occur every time conditions $\pi$ are realized is called a certain event - denoted usually as $U$; an event that can never occur is called an impossible event - usually denoted as $V$.

\section{Sample space}

The sample space $\Lambda$ of a certain random experiment denotes all events, which can occur by the realization of a specified set of conditions $\pi$, i.e. which can be outcomes of the considered experiment. The sample space can be finite (tossing a dice) or infinite (testing a concrete cube in a testing machine). In some cases a system of elementary events can be found, i.e. a system of events that cannot be divided any further (e.g. tossing a dice numbers 1 to 6 represents elementary events). In other cases the system of elementary events is not obvious or does not exist (testing a cube in a testing machine).

All the fundamental terms that have been introduced, experiment, set of conditions $\pi$, event and sample space $\Lambda$ are clarified by the following three simple examples, which constitute an integral part of this summary. Besides a complementary explanation of the relevant terms, the following examples provide further information on the general principles and mathematical tools used to describe real conditions and accepted simplifying assumptions.

\section{Example 2.1}

Tossing a dice is a traditional (and from an educational viewpoint a very useful) example of a random experiment. In this case the set of conditions $\pi$ is trivial. The dice is balanced and symmetrical and cast in a correct way that will not affect the number obtained when the dice is tossed.

The certain event $U$ denotes the event where any of the numbers $1,2,3,4,5$ or 6 occur. etc.).

The impossible event $V$ denotes the event when other numbers appear (e.g. 0, 7, 9

Elementary events $E_{i}, i=1,2$ to 6 , which cannot be further divided, denote the number $i=1,2$ to 6 . If the given set of conditions $\pi$ is satisfied, the occurrence of every elementary event is equally possible. In this case we say that it is a system of equally possible elementary events.

Random events $A_{i}$, for example appearance of the number 1 , can be denoted as $A_{1}=$ $E_{1}$, appearance of the even numbers $A_{2}=E_{2} \cup E_{4} \cup E_{6}$, appearance of the numbers divisible by three, $A_{3}=E_{3} \cup E_{6}$, numbers divisible by two or three $A_{4}=E_{2} \cup E_{3} \cup E_{4} \cup E_{6}$, etc. The sample space $\Lambda$ (i.e. the total of all possible events which may occur at a toss) is, in this case, obviously finite.

\section{Example 2.2}

The cylinder strength of a specific concrete is examined. The random experiment is the loading of a test cylinder in a testing machine. The set of conditions $\pi$ includes the composition, treatment and age of concrete, dimensions of the cube, speed of the loading, etc. The investigated random event is the failure of the concrete cylinder at a certain level of loading. If the loading is sufficiently high, the cylinder always fails, at sufficiently low levels of loading the failure will never occur. At the loading level corresponding to the characteristic cylinder strength of concrete the failure may occur (e.g. 5\% of all cases on average) or may not (e.g. $95 \%$ of all cases).

Elementary events can be defined in many ways, e.g. by a system of intervals of equal width within a certain loading range. Consider concrete of the grade C 20 having the characteristic cylinder strength $20 \mathrm{MPa}$. The possible range of loading from 10 to $50 \mathrm{MPa}$ is 
split into the intervals of $4 \mathrm{MPa}$ and elementary events are defined as a failure of a cylinder at the loading level within each interval. Figure 2.1 shows the results of 50 experiments under specified conditions $\pi$. Solid bars in each interval indicate the number of failed cylinders. It follows from Figure 2.1 that two cylinders failed at the loading level from 18 to $22 \mathrm{MPa}$, nine cylinders failed in the next interval from 22 to $26 \mathrm{MPa}$, seventeen cylinders failed in the interval from 24 to $30 \mathrm{MPa}$, etc. Without a doubt it is not a system of equally possible events (see Figure 2.1). The sample space $\Lambda$ consists of any one-sided or two-sided interval and is obviously infinite.

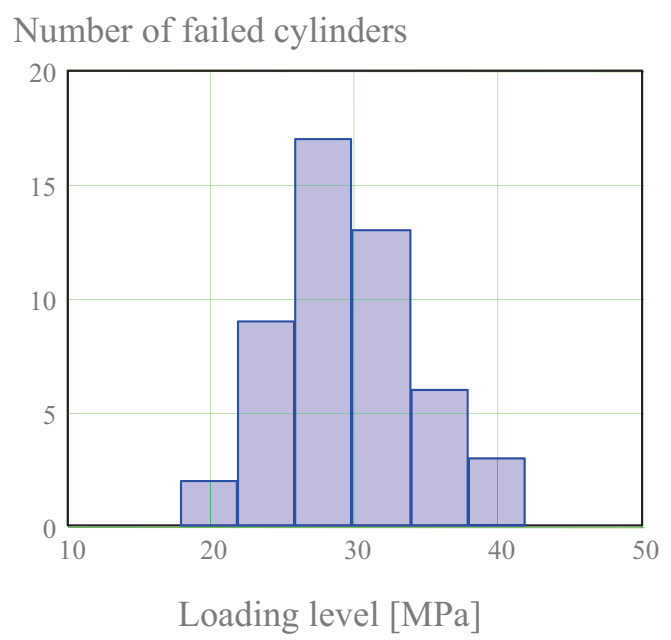

Figure 2.1. The number of failed cylinders versus the loading level.

Figure 2.1 shows a frequently used graphical representation of experimental results, which is referred to as a histogram, and which is commonly used for the development of probabilistic models describing basic variables.

\section{Example 2.3}

Consider throwing a dart onto a board indicated in Figure 2.2. Each throw represents one realization of a random experiment. The set of conditions $\pi$ includes the distance of the board from the throwing point, the size of the board, the type of dart and other conditions for throwing.

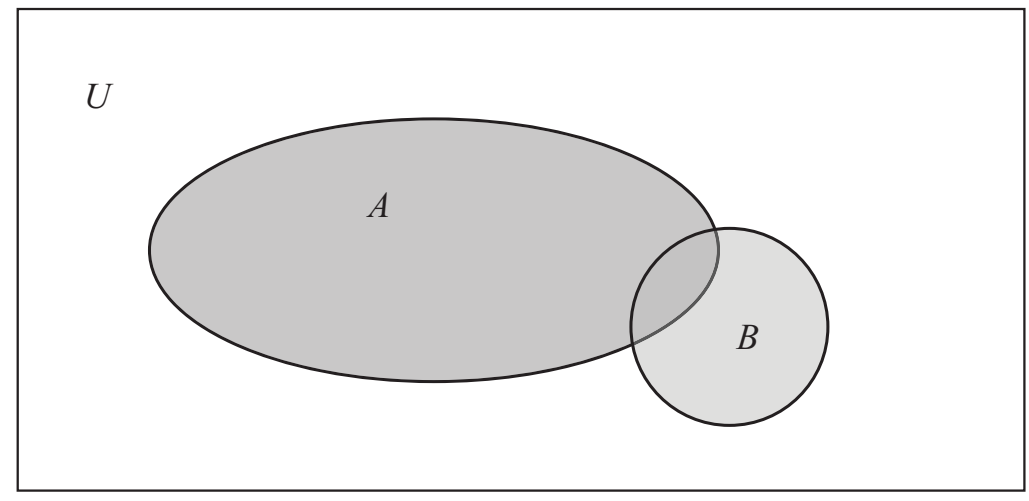

Figure 2.2. An example of throwing a dart onto a board - Venn diagram. 
It is assumed that every point of the board can be hit with equal likelihood and that the board is always hit (these are, undoubtedly, questionable assumptions).

The hitting of the whole board is therefore a certain event $U$.

An impossible event $V$ is a throw that misses the board.

A random event, though, may be the hitting of any smaller area $A$ or $B$ drawn on the board (Figure 2.2) or of any combination of such areas. The system of all the possible areas on the board represents an infinite sample space $\Lambda$.

\subsection{Relations between random events}

The Example 2.3 indicates a common representation of random events (see Figure 2.2) using oval figures illustrating the mutual relations between the random events $A, B, C, \ldots$ (such a representation is called a Venn diagram). In Figure 2.2 the certain event $U$ is represented by the whole rectangle, two random events $A$ and $B$ are symbolized by the ovals. Let us consider some basic relations between events $A$ and $B$, which lead to the definition of other important terms and to the derivation of some general relationships between the random events. Other diagrams similar to Figure 2.2 may illustrate all the following relationships and combinations of random events. A detailed explanation including formal mathematical proofs of all rules may be found in specialised literature $[11,12]$.

If an event $B$ occurs every time the conditions $\pi$ are realized, as a result of which an event $A$ occurs, we say that event $A$ implies event $B$, which is usually symbolically denoted as $A \subset B$. If the events $A$ and $B$ occur simultaneously every time the conditions $\pi$ are realized, we say that an intersection of the two events occurs. An intersection of the events $A$ and $B$ is denoted as $A \cap B$. If at least one of the events $A$ and $B$ occur at every realization of the conditions $\pi$, a union of the two events occurs. This is denoted by $A \cup B$. If event $A$ occurs but event $B$ does not, then the difference $A-B$ of the two events occurs. Events $A$ and $\bar{A}$ are complementary events (we also say that event $\bar{A}$ is the opposite of event $A$ ) if it holds simultaneously that $A \cup \bar{A}=U$ and $A \cap \bar{A}=V$. It can be shown that the following simple rules (the commutative, associative and distributive laws) hold for the intersection and union of random events:

$$
\begin{gathered}
A \cap B=B \cap A, A \cup B=B \cup A \\
(A \cap B) \cap C=A \cap(B \cap C),(A \cup B) \cup C=A \cup(B \cup C) \\
(A \cap B) \cup C=(A \cup C) \cap(B \cup C), A \cup(B \cap C)=(A \cup B) \cap(A \cup C)
\end{gathered}
$$

These basic rules lead to the definition of more complicated relations of the intersection and union of a system of events $A_{\mathrm{i}}$ :

$$
\begin{aligned}
& \bigcap_{i} A_{\mathrm{i}}=A_{1} \cap A_{2} \cap A_{3} \cap \ldots \cap A_{\mathrm{n}} \\
& \cup A_{\mathrm{i}}=A_{1} \cup A_{2} \cup A_{3} \cup \ldots \cup A_{\mathrm{n}}
\end{aligned}
$$

The following rules (so-called de Morgan rules), the validity of which follows from the above relations, are sometimes effectively applied in practical computations of probabilities of complex events

$$
\begin{aligned}
& \overline{\bigcap_{i} A_{i}}=\overline{A_{i}} \cup \overline{A_{2}} \cup \ldots \cup \overline{A_{n}} \\
& \overline{\bigcup_{i} A_{i}}=\overline{A_{1}} \cap \overline{A_{2}} \cap \ldots \cap \overline{A_{n}}
\end{aligned}
$$


The use of these rules is evident from the two following examples.

\section{Example 2.4}

A simple serial system loaded by forces $P$ consists of two elements as shown in Figure 2.3. Failure $F$ of the system may occur due to failure $F_{1}$ of the element 1 or due to failure $F_{2}$ of the element 2 :

$$
F=F_{1} \cup F_{2}
$$

The complementary event $\bar{F}$ (no failure) is, according to relation (2.5), described by an event for which it holds

$$
\bar{F}=\overline{F_{1} \cup F_{2}}=\overline{F_{1}} \cap \overline{F_{2}}
$$

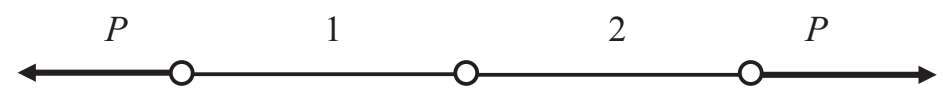

Figure 2.3. A serial system.

\section{Example 2.5}

A town $C$ is supplied with water from two sources $A$ and $B$ by a pipeline, which consists of three independent branches 1,2 and 3 (see the scheme in Figure 2.4). Let us denote $F_{1}$ as the failure of the branch $1, F_{2}$ the failure of the branch 2 and $F_{3}$ the failure of the branch 3. In the case where the sources $A$ and $B$ have sufficient capacity to supply the town $C$, the lack of water in that town is described by the event $\left(F_{1} \cap F_{2}\right) \cup F_{3}$; here, either the branch 3 fails or the branches 1 and 2 fail. For the analysis of this event, however, it may be expedient to study a complementary event describing the sufficiency of water in the town $C$. According to de Morgan's rules the complementary event of the sufficiency of water in the town $C$, is described by the event

$$
\overline{\left(F_{1} \cap F_{2}\right) \cup F_{3}}=\left(\overline{F_{1}} \cup \overline{F_{2}}\right) \cap \overline{F_{3}}
$$

where the event $\left(\overline{F_{1}} \cup \overline{F_{2}}\right)$ represents sufficient water in the join of the branches 1 and 2, which is at the same time the beginning of the branch 3 .

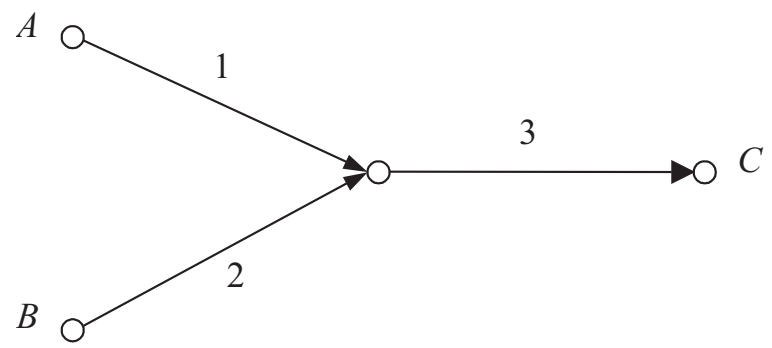

Figure 2.3. Water supply system of a town $C$ from sources $A$ and $B$. 


\section{Example 2.6}

Let us consider a statically determinate truss structure shown in Figure 2.4 consisting of seven members and loaded by forces $P$. The aim is to describe event $F$ as that in which a structural failure occurred. Let $F_{i}$ denote the event that a failure of the element $i=1,2, \ldots, 7$ occurred.

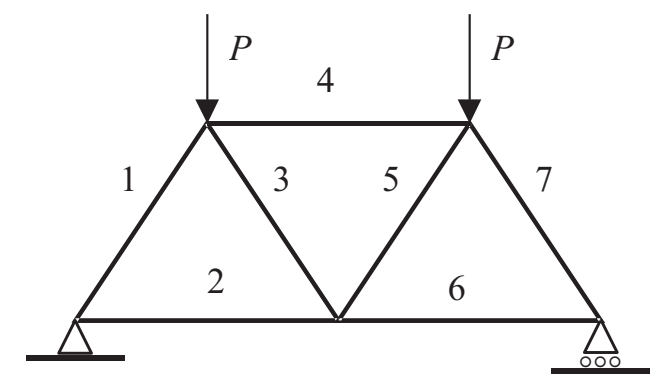

Figure 2.4. Statically determinate truss structure.

The failure of the whole structure (event $F$ ) occurs if a failure of at least one of the members occurs. Therefore it holds that

$$
F=\bigcup_{i=1}^{7} F_{i}
$$

With regard to the conditions of manufacture of the individual members the events $F_{i}$ may be mutually dependent and thus are not exclusive. In the computation of the probability of failure it may then be expedient to consider the complementary event $\bar{F}$ for which it holds, according to de Morgan's rules (2.5)

$$
\bar{F}=\overline{\bigcup_{i=1}^{7} F_{i}}=\bigcap_{i=1}^{7} \overline{F_{i}}
$$

Similar relationships may be effectively used when analysing complex technical systems.

The following additional terms are often used. We say that a system of events $A_{i}$ forms a complete system of events if the union of these events is a certain event $U$. In that case at least one event, $A_{i}$, always occurs. A complete system of mutually exclusive events is another term that is sometimes used when analysing complex events. In that case only one event $A_{i}$ always occurs.

\subsection{Definitions of Probability}

\section{Classical definition}

Probability describes the occurrence of random events. The definition of probability is, however, a mathematically intricate problem. Historically it experienced an interesting evolution, reflecting the remarkable development of the theory of probability and its practical applications. The classical definition of probability is based on a complete system of elementary events. Let an event $A$ consist of $m$ out of $n$ equally likely elementary events where the total number $n$ is formed by a complete system of mutually exclusive events. The probability of the event $A$ is then given by the quotient 


$$
\mathrm{P}(A)=m / n
$$

For probability defined in this way it obviously holds that

$$
\begin{gathered}
0 \leq \mathrm{P}(A)=m / n \leq 1 \\
\mathrm{P}(U)=n / n=1, \mathrm{P}(V)=0 / n=0
\end{gathered}
$$

It can also be shown for a system of mutually exclusive events $A_{i}$ that the probability of the union of these events is given by the relation

$$
\mathrm{P}\left[\bigcup_{i=1}^{\infty} A_{i}\right]=\sum_{i=1}^{\infty} \mathrm{P}\left[A_{i}\right]
$$

The classical definition of probability is fully acceptable for many elementary cases such as the tossing of a dice in Example 2.1. However, if the dice is not symmetrical, the classical definition obviously fails. Examples 2.2 and 2.3 further indicate that a finite system of elementary events is not sufficient for fundamental problems of civil engineering. In the attempt to deal with these insufficiencies other definitions of probability gradually emerged.

\section{Geometric definition}

The geometrical definition of probability is related to the throwing of a dart in Example 2.3. According to this definition the probability of an event $A$ is equal to the quotient of the surface area of event $A$, denoted $\operatorname{area}(A)$, and of the surface area of the certain event $U$ denoted $\operatorname{area}(U)$, i.e. by the quotient

$$
\mathrm{P}(A)=\operatorname{area}(A) / \operatorname{area}(U)
$$

Thus, the geometric definition attempts to eliminate one insufficiency of the classical definition, which lies in the finite number of elementary events. However, this definition still does not take into account the reality that not all the points on the board (event $U$ ) have the same possibility of occurrence. Obviously, the "surface area" is not an appropriate measure of occurrence; this difficulty is still to be solved.

\section{Statistical definition}

The statistical definition of probability is based on results of an experiment repeated many times. Let us consider a sequence of $n$ realizations of an experiment. Assume that a certain event $A$ comes up $m(A)$ times out of these $n$ experiments. It appears that the relative frequency of the occurrence of the event $A$, i.e. the fraction $m(A) / n$, attains an almost constant value; with an increasing number of realisations $n$. This phenomenon is called the statistical stability of relative frequencies, i.e. of the fraction $m(A) / n$. The value to which the relative frequency $m(A) / n$ approaches as $n$ increases $(n \rightarrow \infty)$ is accepted as an objective measure of the occurrence of the event $A$ and is called the probability $\mathrm{P}(A)$ of the event $A$ :

$$
\mathrm{P}(A)=\lim _{n \rightarrow \infty} \frac{m(A)}{n}
$$

However, the assumption of statistical stability and convergence indicated in equation (2.11) (i.e. the limit of the quantity derived from the results of experiments) causes some mathematical difficulties.

\section{Axiomatic definition}

The classical, geometrical as well as statistical definitions of probability attempt to define not only the probability, but also to propose a rule of its computation. Such requirement is evidently too hard to meet (if not impossible). 
The long-term effort to define the basic terms of the theory of probability seems to be completed by the so-called axiomatic system, which is accepted all over the world. The axiomatic system defines only the term of probability and its fundamental properties without providing any practical instructions for its determination.

Note that equations (2.7) to (2.9) characterize the common properties of the classical, geometrical as well as statistical definition of probability:

1. the probability of a certain event is equal to 1 ;

2. the probability of an impossible event is equal to 0 ; and

3. if an event $A$ is a union of partial and mutually exclusive events $A_{1}, A_{2}, \ldots, A_{n}$, then the probability of event $A$ is equal to the sum of probabilities of the partial events.

The axiomatic definition of probability introduces these general properties as axioms. Probability $P$ is a real function, defined in a sample space $\Lambda$ above the certain event $U$ with these properties:

1. If $A \in \Lambda$, then

$$
P(A)=\geq 0
$$

2. For the certain event $U$, it holds that

$$
\mathrm{P}(U)=1
$$

3. If $A_{i} \in \Lambda, i=1,2, \ldots$ and if for arbitrary $i$ and $j A_{i} \cap A_{j}=V$, then

$$
\mathrm{P}\left(\bigcup_{i=1}^{\infty} A_{i}\right)=\sum_{i=1}^{\infty} \mathrm{P}\left(A_{i}\right)
$$

It can be shown that the above-mentioned three axioms are satisfied by the classical, geometric and statistical definitions. Moreover, the axiomatic definition of probability also fits the new concept of probability as a measure of the fulfilment of a statement or requirement, often assessed only by intuition and a subjective view (an expert judgment). Thus, in such cases the concept of reproducible (repeatable) random events, which forms the basis for the probability determination of an event, is completely impossible.

Note that using the above axioms the modern theory of probability transfers into the general theory of sets. Probability is then defined as a non-negative additive function of sets, which can be interpreted as a generalization of the term "surface area" in the geometric definition of probability.

\subsection{Basic rules for the computation of probabilities}

Using equations (2.6) to (2.9) or axioms (2.12) to (2.14) other rules, which can be useful in computations of probabilities, can be derived. If $A_{i}, i=1,2, \ldots n$, form a complete system of events, then it evidently holds that

$$
\mathrm{P}\left(\bigcup_{i=1}^{n} A_{i}\right)=\mathrm{P}(U)=1
$$

If an event $A$ is a union of partial and mutually exclusive events, $A_{i}, i=1,2, \ldots, n$, we can write

$$
\mathrm{P}(A)=\mathrm{P}\left(\bigcup_{i=1}^{n} A_{i}\right)=\sum_{i=1}^{n} \mathrm{P}\left(A_{i}\right)
$$

For the probability of the union of two arbitrary events $A$ and $B$ (which do not have to be exclusive) the principle of summation of probabilities holds

$$
\mathrm{P}(A \cup \mathrm{B})=\mathrm{P}(A)+\mathrm{P}(B)-\mathrm{P}(A \cap B)
$$


which follows from (2.16) for mutually exclusive events $A$ and $B-(A \cap B)$, of which the union is the studied event $A \cup B$.

If $A_{i}, i=1,2, \ldots, n$, is a complete system of mutually exclusive events, then we obtain from equation (2.15)

$$
\mathrm{P}\left(\bigcup_{i=1}^{n} A_{i}\right)=\sum_{i=1}^{n} \mathrm{P}\left(A_{i}\right)=\mathrm{P}(U)=1
$$

From equation (2.18) for complementary events $A$ and $\bar{A}$ it follows that

$$
\mathrm{P}(\bar{A})=1-\mathrm{P}(A)
$$

\section{Example 2.7}

Let us determine the probability that a serial system described in Example 2.4 will fail. Let $\mathrm{P}\left(F_{1}\right)=0,05, \mathrm{P}\left(F_{2}\right)=0,05$ and $\mathrm{P}\left(F_{1} \cap F_{2}\right)=0,02$. Then, considering the relation (2.17) we find that

$$
\mathrm{P}\left(F_{1} \cup F_{2}\right)=\mathrm{P}\left(F_{1}\right)+\mathrm{P}\left(F_{2}\right)-\mathrm{P}\left(F_{1} \cap F_{2}\right)=0,05+0,05-0.02=0,08
$$

Note that the events $F_{1}$ and $F_{2}$ are not exclusive (failures of both elements can occur simultaneously). If they were exclusive, the probability of failure would be 0,10 . Other details concerning this example will be provided in the next section by the principle of multiplication of probabilities.

\subsection{Conditional probability}

Conditional probability $\mathrm{P}(A \mid B)$ of the event $A$ under a complementary condition that another event $B$ has occurred simultaneously (or before), and has a non-zero probability, is an important concept in the contemporary theory of probability which is often used in the theory of structural reliability. The conditional probability $\mathrm{P}(A \mid B)$ is defined as the fraction

$$
\mathrm{P}(A \mid B)=\mathrm{P}(A \cap B) / \mathrm{P}(B)
$$

This relation is the basis of the so-called Bayes concept of the theory of probability (Thomas Bayes (1702 to 1761)). For two special cases important simplifications of relation (2.20) are valid. If events $A$ and $B$ are exclusive, i.e. $A \cap B=V$, then $\mathrm{P}(A \mid B)=0$; if an event $A$ implies an event $B$, i.e. it holds that $A \subset B$, then $\mathrm{P}(A \mid B)=\mathrm{P}(A) / \mathrm{P}(B)$. If $B \subset A$, then $\mathrm{P}(A \mid B)=$ 1. These rules follow directly from the basic properties of probability described in sections 2.2 and 2.3.

A general rule for the multiplication of probabilities follows from equation (2.20)

$$
\mathrm{P}(A \cap B)=\mathrm{P}(B) \mathrm{P}(A \mid B)
$$

Consider again the special cases. If the events $A$ and $B$ are exclusive, i.e. $A \cap B=V$, then $\mathrm{P}(A \mid B)=0$ and also $\mathrm{P}(A \cap B)=0$; if $A \subset B$, then $\mathrm{P}(A \mid B)=\mathrm{P}(A) / \mathrm{P}(B)$ and $\mathrm{P}(A \cap B)=$ $\mathrm{P}(B)$; if, conversely, $B \subset A$, then $\mathrm{P}(A \mid B)=1$ and $\mathrm{P}(A \cap B)=\mathrm{P}(B)$.

We say that events $A$ and $B$ are independent (the occurrence of event $B$ does not influence the probability of the occurrence of event $A$ ) if it holds that $\mathrm{P}(A \mid B)=\mathrm{P}(A)$. Consider the special cases introduced above. If events $A$ and $B$ are exclusive, then they are dependent because $\mathrm{P}(A \mid B)=0 \neq \mathrm{P}(A)$ (if $A$ is not an impossible event). If $A \subset B$, then $A$ and $B$ are dependent events, because $\mathrm{P}(A \mid B)=\mathrm{P}(A) / \mathrm{P}(B) \neq \mathrm{P}(A)$, if conversely $B \subset A$, then the events $A$ and $B$ are dependent, because $\mathrm{P}(A \mid B)=1 \neq \mathrm{P}(A)$. Therefore independent events $A$ and $B$ must not be exclusive, i.e. $A \cap B \neq V$, and satisfy the trivial relations $A \not \subset B$ and $B \not \subset A$. 
If two events $A$ and $B$ are independent (and therefore it holds that $A \cap B \neq V, A \not \subset B$ and $B \not \subset A)$, then it follows from equation (2.21)

$$
\mathrm{P}(A \cap B)=\mathrm{P}(A) \mathrm{P}(B)
$$

Relation (2.22) is the principle of the multiplication of probabilities, according to which the probability of intersection (a simultaneous occurrence of two independent random events) is given by the product of their probabilities. This fundamental rule is needed for probability integration in the theory of reliability.

\section{Example 2.8}

Taking into account relation (2.21) the following relation can be written for the probability of failure of a serial system described in Example 2.7

$$
\mathrm{P}(F)=\mathrm{P}\left(F_{1} \cup F_{2}\right)=\mathrm{P}\left(F_{1}\right)+\mathrm{P}\left(F_{2}\right)-\mathrm{P}\left(F_{1} \cap F_{2}\right)=\mathrm{P}\left(F_{1}\right)+\mathrm{P}\left(F_{2}\right)-\mathrm{P}\left(F_{1}\right) \mathrm{P}\left(F_{2} \mid F_{1}\right)=0,10-0,05 \mathrm{P}\left(F_{2} \mid F_{1}\right)
$$

If the events $F_{1}$ and $F_{2}$ are independent, then $\mathrm{P}\left(F_{2} \mid F_{1}\right)=\mathrm{P}\left(F_{2}\right)$ and the failure probability is given as

$$
\mathrm{P}(F)=\mathrm{P}\left(F_{1} \cup F_{2}\right)=\mathrm{P}\left(F_{1}\right)+\mathrm{P}\left(F_{2}\right)-\mathrm{P}\left(F_{1}\right) \mathrm{P}\left(F_{2}\right)=0,10-0,0025=0,0975
$$

If the events $F_{1}$ and $F_{2}$ are perfectly dependent $\left(F_{1} \subset F_{2}\right)$, i.e. $\mathrm{P}\left(F_{2} \mid F_{1}\right)=1$, then

$$
\mathrm{P}(F)=\mathrm{P}\left(F_{1} \cup F_{2}\right)=\mathrm{P}\left(F_{1}\right)+\mathrm{P}\left(F_{2}\right)-\mathrm{P}\left(F_{1}\right)=0,10-0,05=0,05
$$

The serial system acts in this case as a single element. Thus, in general, the probability of failure of the considered serial system fluctuates from 0,05 to 0,0975 depending on the degree of dependence of the events $F_{1}$ and $F_{2}$.

Assume that an event $A$ can occur only by the realization of one of the mutually exclusive events $B_{i}, i=1,2, \ldots, n\left(n=5\right.$ in Figure 2.5), for which the probabilities $\mathrm{P}\left(B_{i}\right)$ are known. If the conditional probabilities $\mathrm{P}\left(A \mid B_{i}\right)$ are also known (obviously $\mathrm{P}\left(A \mid B_{5}\right)=0$ in Figure 2.5), then the probability of the event $A$ can be assessed using the relation

$$
\mathrm{P}(A)=\sum_{i=1}^{n} \mathrm{P}\left(B_{i}\right) \mathrm{P}\left(A \mid B_{i}\right)
$$

which is called the theorem of the total probability.

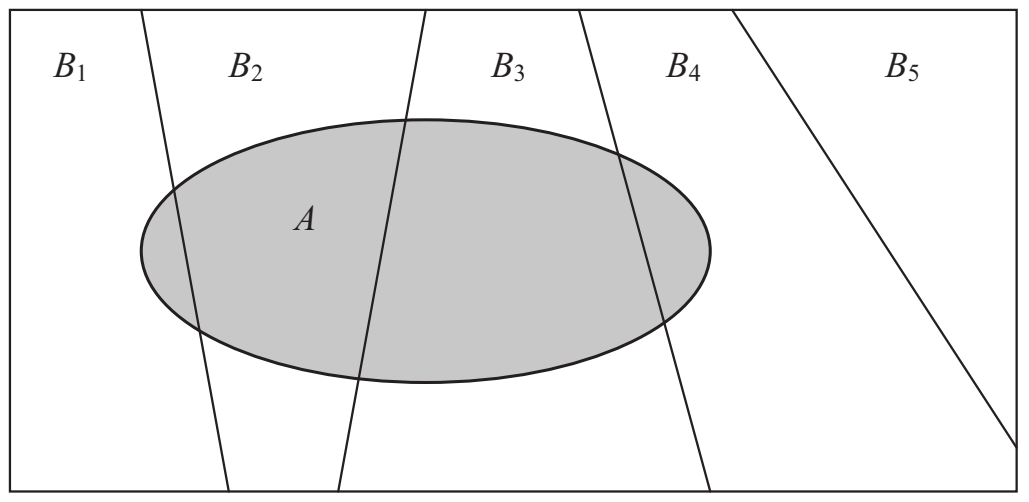

Figure 2.5. An event $A$ and mutually exclusive events $B_{i}$ 


\subsection{Bayes' theorem}

When an event $A$ occurs, it is natural to investigate which of the events $B_{i}$ caused $A$, i.e. what is the probability of the individual hypotheses $B_{i}$ assuming that A occurred (see Figure 2.5), which is denoted as the probability $\mathrm{P}\left(B_{i} \mid A\right)$. A very important relation follows from relations (2.20), (2.21) and (2.23)

$$
\mathrm{P}\left(B_{i} \mid A\right)=\frac{\mathrm{P}\left(B_{i}\right) \mathrm{P}\left(A \mid B_{i}\right)}{\sum_{i=1}^{n} \mathrm{P}\left(B_{j}\right) \mathrm{P}\left(A \mid B_{j}\right)}
$$

which is often referred to as the Bayes rule or theorem.

The following important task of the theory of structural reliability illustrates the general procedure of practical application of the Bayes rule. If a failure of a structure, denoted as the event $A$, can be caused by one of the hypotheses $B_{i}$ whose probabilities of occurrence $\mathrm{P}\left(B_{i}\right)$ are known from previous experience, and if the conditional probabilities $\mathrm{P}\left(A \mid B_{i}\right)$, that the failure $A$ occurred in consequence of a hypothesis $B_{i}$, are also known, then the probability of failure $\mathrm{P}(A)$ can be determined from the principle of the total probability described by equation (2.23).

If, however, the failure $A$ did occur, i.e. the event $A$ occurred, then the probabilities of the individual hypotheses, which could have caused the failure, are of importance. We are therefore interested in the conditional probabilities $\mathrm{P}\left(B_{i} \mid A\right)$, which can be determined using the Bayes rule (2.24).

Practical use of relations (2.23) and (2.24) is illustrated by the following examples.

\section{Example 2.9}

For the assessment of an existing reinforced concrete structure control tests are available, which indicate that the actual strength is lower than the characteristic value $20 \mathrm{MPa}$ (event $B_{1}$ ) with the probability $p_{1}{ }^{\prime}=\mathrm{P}\left(B_{1}\right)=0,05$ and greater than $20 \mathrm{MPa}$ (event $B_{2}$ ) with the probability $p_{2}{ }^{\prime}=\mathrm{P}\left(B_{2}\right)=0,95$. For the subsequent verification of the concrete strength an inaccurate non-destructive method was used. Let $A$ denote the event that the concrete strength assessed by the non-destructive method is greater than $20 \mathrm{MPa}$. Assume that errors of the non-destructive method can be expressed by conditional probabilities

$$
\mathrm{P}\left(A \mid B_{1}\right)=0,30, \mathrm{P}\left(A \mid B_{2}\right)=0,90
$$

Thus, due to the inaccuracy of the non-destructive method a concrete with a strength lower than $20 \mathrm{MPa}$ can be considered as a concrete with a strength greater than $20 \mathrm{MPa}$ with a the probability of 0,30 and a concrete of a strength greater than $20 \mathrm{MPa}$ is considered as such only with the probability of 0,90 .

The probability of the occurrence of the event $A$ (a non-destructive strength is greater than $20 \mathrm{MPa}$ ) follows from the principle of complete probability (2.23)

$$
\mathrm{P}(A)=\sum_{i=1}^{2} \mathrm{P}\left(B_{i}\right) \mathrm{P}\left(A \mid B_{i}\right)=0,05 * 0,30+0,95 * 0,90=0,87
$$

This means that using the inaccurate non-destructive method, the concrete strength greater than $20 \mathrm{MPa}$ will be predicted with the probability 0,87 . Note that if the nondestructive tests were absolutely accurate, e.g. the conditional probabilities were

$$
\mathrm{P}\left(A \mid B_{1}\right)=0, \mathrm{P}\left(A \mid B_{2}\right)=1
$$

it would follow from equation (2.23) 


$$
\mathrm{P}(A)=\sum_{i=1}^{2} \mathrm{P}\left(B_{i}\right) \mathrm{P}\left(A \mid B_{i}\right)=0,05 * 0+0,95 * 1=0,95
$$

However, from a practical point of view another question is more important; what is the probability $\mathrm{P}\left(B_{2} \mid A\right)$ of the hypothesis $B_{2}$, that a concrete for which the non destructive test indicated the strength greater than $20 \mathrm{MPa}$ (and thus the event $A$ occurred) really does have a strength greater than $20 \mathrm{MPa}$ (and thus the event $B_{2}$ occurs)? This probability can be assessed directly from the Bayes rule (2.24) for the probability of hypotheses

$$
\mathrm{P}\left(B_{2} \mid A\right)=\frac{\mathrm{P}\left(B_{2}\right) \mathrm{P}\left(A \mid B_{2}\right)}{\sum_{i=1}^{2} \mathrm{P}\left(B_{j}\right) \mathrm{P}\left(A \mid B_{j}\right)}=\frac{0,95 * 0,90}{0,05 * 0,30+0,95 * 0,90}=0,98
$$

Thus, if the strength is greater than $20 \mathrm{MPa}$ according to the non-destructive test, then the probability that the concrete has really the strength greater than $20 \mathrm{MPa}$ increases from the original value of 0,95 to 0,98 .

Bayes' rule is widely applied in many other practical situations in engineering practice, e.g. in situations where prior (previous) information about the distribution of probabilities is updated with regard to newly acquired knowledge. This important procedure of probability updating is described in the following section.

\subsection{Updating of probabilities}

Bayes' rule (2.24) is often applied in the so-called updating of the distribution of probabilities, which is based on random experiments (often repeated) isolated in time. Similarly, as in section 2.5 , it is assumed that probabilities $\mathrm{P}\left(B_{i}\right)$ are known from previous experience (sometimes remote, vague or merely subjective). That is why they are called original (a priori) probabilities and they are denoted simply as $p_{i}{ }^{\prime}=\mathrm{P}\left(B_{i}\right)$.

At present, after some time, experiments are carried out to determine the conditional probabilities $\mathrm{P}\left(A \mid B_{i}\right)$ of the studied event $A$ under the condition that the event $B_{i}$ occurred, which can be considered as the measures of likelihood that the cause of the event $A$ was the very event $B_{i}$. These conditional probabilities, or values proportional to them, are therefore called likelihoods $l_{\mathrm{i}} \propto \mathrm{P}\left(A \mid B_{i}\right)$; the symbol $\propto$ means "proportional to" (likelihoods $l_{\mathrm{i}}$ thus need not be necessarily normalized to the conventional interval $<0,1>$ ). We are inquiring about updated (a posteriori) probabilities $p{ }^{\prime}{ }_{i}=\mathrm{P}\left(B_{i} \mid A\right)$ of the event (i.e. hypothesis) $B_{i}$ updated in respect of the result of a new experiment (of the event $A$ ). An important relation for $p{ }^{\prime}{ }_{i}$ follows directly from the Bayes rule (2.24):

$$
p_{i}^{\prime \prime}=\frac{p_{i}^{\prime} l_{i}}{\sum_{j} p_{j}^{\prime} l_{j}}
$$

Formula (2.25) obviously holds generally for likelihoods which, unlike probabilities, are not normalized to the interval $\langle 0,1\rangle$ and only express the relative contribution of the events (hypotheses) $B_{i}$ on the observed event $A$.

Relation (2.25) is a basis for updating of probabilities, which is often applied in many engineering procedures, particularly in the assessment of existing structures. It is in these cases that present information is combined with previous (often subjective) information, i.e. with information about a structure at various points in time, usually quite remote. This is the reason it is necessary to verify the conditions under which the previous information was 
obtained and to resist the temptation to apply inhomogeneous data, which may be misleading and could lead to serious mistakes and misunderstandings.

\section{Example 2.10}

Consider again the reinforced concrete structure described in Example 2.9. We observe that from previous control tests original (a priori) probabilities are known: $p_{1}{ }^{\prime}=$ $\mathrm{P}\left(B_{1}\right)=0,05$ (the probability that the real strength is lower than the characteristic value of 20 $\mathrm{MPa}$, which is event $\left.B_{1}\right)$ and $p_{2}{ }^{\prime}=\mathrm{P}\left(B_{2}\right)=0,95$ (the probability that the real strength is greater than $20 \mathrm{MPa}$, event $B_{2}$ ).

In the subsequent assessment of the structure supplementary tests of concrete strength were carried out using core samples, which is sufficiently accurate (unlike the non-destructive tests from previous Example 2.9). Thus in analysing the results it is not necessary to consider the inaccuracies. These tests suggest that the likelihood of the event $B_{1}$ is $l_{1} \propto \mathrm{P}\left(A \mid B_{1}\right)=0,2$ and the likelihood of the event $B_{2}$ is $l_{2} \propto \mathrm{P}\left(A \mid B_{2}\right)=0,8$ (the introduced likelihoods are already normalized). Updated (a posteriori) probabilities follow from relation (2.25)

$$
\begin{aligned}
& p_{1}^{\prime \prime}=\frac{p_{1}^{\prime} l_{1}}{\sum_{j=1}^{2} p_{j}^{\prime} l_{j}}=\frac{0,05 * 0,20}{0,05 * 0,20+0,95 * 0,80}=0,01 \\
& p_{2}^{\prime \prime}=\frac{p_{2}^{\prime} l_{2}}{\sum_{j=1}^{2} p_{j}^{\prime} l_{j}}=\frac{0,95 * 0,80}{0,05 * 0,20+0,95 * 0,80}=0,99
\end{aligned}
$$

Thus the updated (a posteriori) distribution of probabilities $p_{i}$ " is more favourable than the original (a priori) distribution of probabilities $p_{i}$.

Note that when the supplementary tests suggest that the likelihoods of both the events $B_{1}$ and $B_{2}$ are equal, e.g. $l_{1}=\mathrm{P}\left(A \mid B_{1}\right)=l_{2}=\mathrm{P}\left(A \mid B_{2}\right)=0,5$, the updated probabilities equal the original ones $\left(p_{i}{ }^{\prime}=p_{i}{ }^{\prime}\right)$. If, however, the analysis of the event $A$ showed that the likelihood of the event $B_{1}$ is greater than the likelihood of the event $B_{2}$, e.g. $l_{1} \propto \mathrm{P}\left(A \mid B_{1}\right)=0,7$ and $l_{2} \propto$ $\mathrm{P}\left(A \mid B_{2}\right)=0,3$, the a posteriori probabilities change significantly:

$$
\begin{aligned}
& p_{1}^{\prime \prime}=\frac{p_{1}^{\prime} l_{1}}{\sum_{j=1}^{2} p_{j}^{\prime} l_{j}}=\frac{0,05 * 0,70}{0,05 * 0,70+0,95 * 0,30}=0,11 \\
& p_{2}^{\prime \prime}=\frac{p_{2}^{\prime} l_{2}}{\sum_{j=1}^{2} p_{j}^{\prime} l_{j}}=\frac{0,95 * 0,30}{0,05 * 0,70+0,95 * 0,30}=0,89
\end{aligned}
$$

The updated (a posteriori) distribution of probabilities $p_{i}$ " is then less favourable than the original (a priori) distribution $p_{i}{ }^{\prime}$. However, the influence of the a priori distribution still seems to prevail; it disappears only in the case of an extreme distribution of likelihoods, e.g. when $l_{1}$ approaches one $\left(l_{1} \propto \mathrm{P}\left(A \mid B_{1}\right) \rightarrow 1\right)$ and at the same time $l_{2}$ approaches zero $\left(l_{2} \propto\right.$ $\left.\mathrm{P}\left(A \mid B_{2}\right) \rightarrow 0\right)$. But, in practice, the distribution of likelihoods is usually similar to the distribution of a priori probabilities.

\section{Example 2.11}

Tensile components of an existing structure were designed for the load of $2 \mathrm{kN}$. After a reconstruction, the load on each of these components is increased up to 2,5 kN. Prior experience shows that the elements are able to resist the load of $2,5 \mathrm{kN}$ (an event $B$ ) with a probability $p_{1}{ }^{\prime}=\mathrm{P}(B)=0,8$ and they fail with a probability $p_{2}{ }^{\prime}=\mathrm{P}(\bar{B})=0,2$. Furthermore, it 
is known from prior experience that half of these components cannot resist the load of 2,5 $\mathrm{N}$ and are able to bear a lower load of $2,3 \mathrm{kN}$ (an event $A$ ). Knowing this, the probability $p_{1}{ }^{\prime}=$ $\mathrm{P}(B)=0,8$ can be updated by testing one of these components up to $2,3 \mathrm{kN}$.

Let us suppose that the test was successful, i.e. the element did not fail with the $2,3 \mathrm{kN}$ load. The likelihood of the event $B$, i.e. $l_{1} \propto \mathrm{P}(A \mid B)=1$, and of the event $\bar{B}$, i.e. $l_{2} \propto \mathrm{P}(A \mid \bar{B})$ $=0,5$, were estimated from the result of this test. Then an a posteriori probability follows from relation (2.25),

$$
p_{1}^{\prime \prime}=\frac{p_{1}^{\prime} l_{1}}{\sum_{j=1}^{2} p_{j}^{\prime} l_{j}}=\frac{0,80 * 0,10}{0,80 * 1,0+0,20 * 0,5}=0,89
$$

Thus the a priori probability $p_{1}^{\prime}=0,8$ is updated to the value $p_{1} "=0,89$. The updating of probabilities can now be repeated by another test where the a posteriori probability obtained in the previous step will be considered as a priori information. If the other test was also successful, then the new a posteriori probability would be

$$
p_{1}^{\prime \prime}=\frac{p_{1}^{\prime} l_{1}}{\sum_{j=1}^{2} p_{j}^{\prime} l_{j}}=\frac{0,89 * 1,0}{0,89 * 1,0+0,11 * 0,5}=0,94
$$

This repetitive procedure of updating probabilities is quite characteristic in practical applications.

However, what happens when the first test is not successful? If the likelihoods $l_{1}$ and $l_{2}$ are estimated in this case as $l_{1} \propto \mathrm{P}(A \mid B)=0,5$ and $l_{2} \propto \mathrm{P}(A \mid \bar{B})=1,0$, it follows for the $a$ posteriori probability $p{ }^{\prime}{ }_{1}$

$$
p_{1}^{\prime \prime}=\frac{p_{1}^{\prime} l_{1}}{\sum_{j=1}^{2} p_{j}^{\prime} l_{j}}=\frac{0,80 * 0,5}{0,80 * 0,5+0,20 * 1,0}=0,67
$$

which is an unfavourable reduction of the original (a priori) value $p_{1}{ }^{\prime}=0,8$. In that case it may be useful to execute additional tests and repeat the updating. 
This chapter provides a concise description of the most important probabilistic models of random variables with regard to their applications in structural reliability. A detailed description of these and other less frequently used theoretical models of random variables can be found in the International Standards ISO $[3,4,5]$ and specialised literature $[11,12,14,17$, $18]$.

\subsection{Random variable}

A random variable $X$ is defined as a variable that attains one and only one value $x$, which is unknown in advance, when a set of conditions $\pi$ is realised, i.e. when a certain random event occurs (see section 2.1). Thus the random variable may also be defined as an indicator of random events caused by specified experiments.

An example of the random variable is a force at the failure of a concrete cylinder loaded under given conditions in a testing machine (see Example 2.2). Random variables are usually denoted by upper-case letters, e.g. $X, Y, \ldots$, and their particular values (realisations, e.g. a given force at the failure of a concrete cylinder) by lower-case letters, e.g. $x, y, \ldots$ In practical applications this notation rule may become cumbersome and is not always convenient.

In structural reliability both continuous random variables (attaining any values of a given interval or domain) and discrete random variables (attaining distinct values only) are used. The following summary is focused on the most important continuous variables, which are commonly applied to describe actions, material properties, and geometric data. Information about discrete random variables and their application can be found in specialised literature $[11,12]$.

The totality of all the possible realisations $x$ of a considered random variable $X$ is called a population. It is described by a distribution of probabilities, i.e. by a function determining the probability that a random variable $X$ attains a given set of values (in the case of continuous variables, e.g. strength of concrete cylinders) or attains a distinct value (in the case of discrete variables, e.g. a number on dice).

The distribution function $\Phi(x)$ (sometimes denoted as $\left.\Phi_{X}(x)\right)$ gives for each value $x$ a probability that the random variable $X$ will be less than or equal to $x$, thus

$$
\Phi(x)=\mathrm{P}(X \leq x)
$$

The probability density function $\varphi(x)$ of a continuous random variable $x$ is the derivative (if it exists) of the distribution function

$$
\varphi(x)=\frac{\mathrm{d} \Phi(x)}{\mathrm{d} x}
$$

The following example illustrates a mutual relation between the distribution function $\Phi(x)$ and the probabilistic density function $\varphi(x)$.

\section{Example 3.1}

A continuous random variable $X$, which may attain any point $x$ within an interval $<a$, $b>$ with equal likelihood (each point $x$ has the same probability density $\varphi(x)$ ) is described by a so-called uniform distribution shown in Figure 3.1. The uniform distribution is a basic type of distribution of great theoretical significance applicable also as a theoretical model for some 
specific actions (for example the weight of subway car) or geometric data. In addition, the uniform distribution is used as an auxiliary tool in simulation of continuous random variables having a certain type of distribution function.

The distribution function $\Phi(x)$ and the probability density function $\varphi(x)$ for the uniform distribution are shown in Figure 3.1.

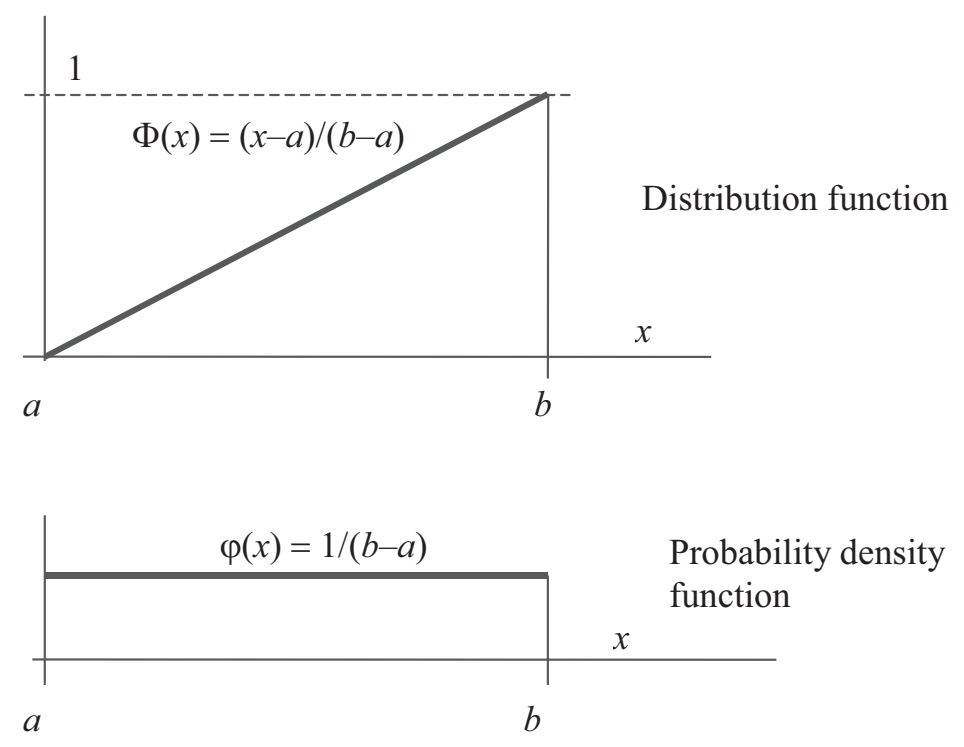

Figure 3.1. Uniform distribution.

Figure 3.1 clearly illustrates the mutual relation of the distribution function $\Phi(x)$ and probability density function $\varphi(x)$ defined by equations (3.1) and (3.2). It also indicates the general property of the probability density function $\varphi(x)$ following from equations (3.1) and (3.2): the integral of the probability density function $\varphi(x)$ within its definition domain is equal to 1

$$
\int_{-\infty}^{\infty} \varphi(x) \mathrm{d} x=\int_{a}^{b} \varphi(x) \mathrm{d} x=1
$$

In other words, the probability of all the possible values of a random variable (given by the above integral) is 1 . Thus the surface surrounded by the horizontal axis $x$ and the density function $\varphi(x)$ within the definition domain has a unit area.

In addition to the distribution function $\Phi(x)$ and probability density function $\varphi(x)$, the random variable $X$ may also be described by various parameters. The most frequently used are so-called moment parameters. The fundamental moment parameter, which describes the location of a population, is the mean $\mu$ (also called the expectation, expected value or in common technical terminology the average). The mean $\mu$ is defined by the moment of the first order about the origin, thus

$$
\mu=\int x \varphi(x) \mathrm{d} x
$$

Geometrically, the mean $\mu$ coincides with the $x$ coordinate of the centre of gravity of the surface surrounded by the horizontal axis $x$ and the density function $\varphi(x)$. Figure 3.2 
shows an example of a bell-shaped probability density function (of a log-normal distribution $\operatorname{LN}(1 ; 0,2 ; 1)$ having the mean $\mu=1,0$, the standard deviation $\sigma=0,2$, and the skewness $\alpha=$ 1,0 described in the following sections) illustrating the geometric interpretation of the mean $\mu$.

The measure of dispersion of a random variable $X$ relative to the mean $\mu$ is given by the central moment of the second order (the moment of inertia of the area bounded by the horizontal axis $x$ and the curve of probability density function $\varphi(x)$ ), which is called the variance $\sigma^{2}$ and is

$$
\sigma^{2}=\int(x-\mu)^{2} \varphi(x) \mathrm{d} x
$$

Probability density $\varphi(x)$

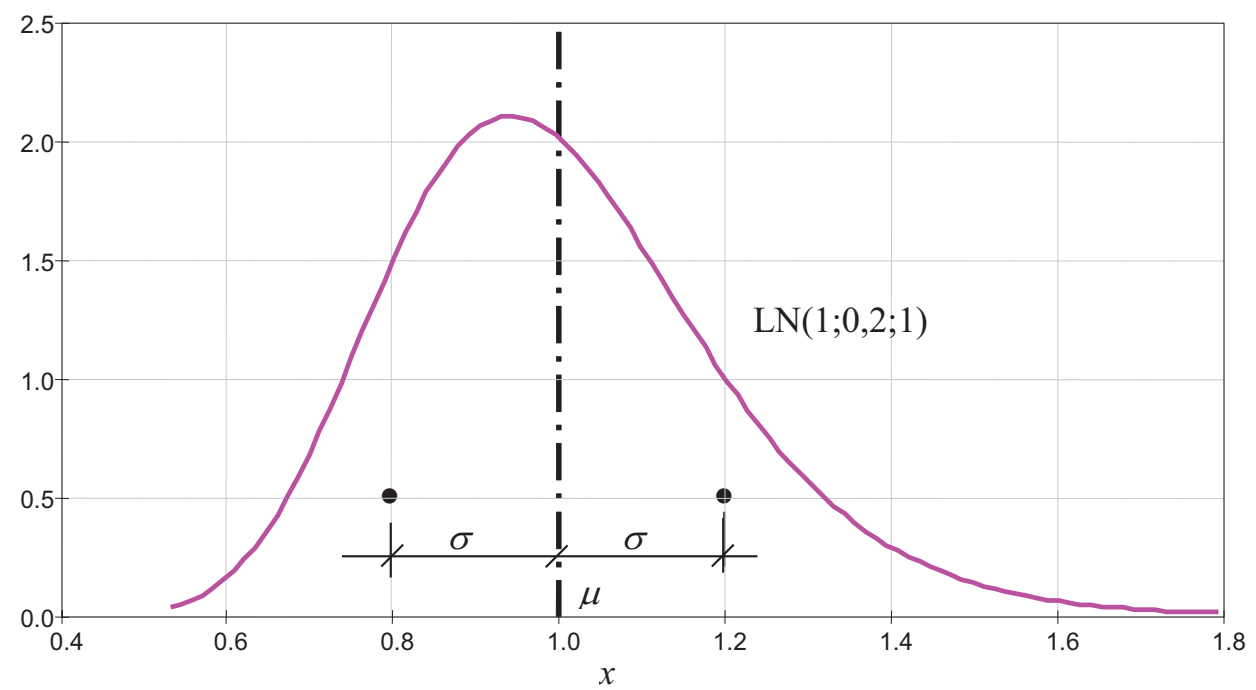

Figure 3.2. Geometric illustration of the mean $\mu=1,0$ and the standard deviation $\sigma=0,2$.

The square root of the variance $\sqrt{\sigma^{2}}=\sigma$ denotes the standard deviation of the random variable (radius of inertia, of the area bounded by the horizontal axis $x$ and the curve of probability density function $\varphi(x)$ ). Figure 3.2 illustrates the geometric interpretation of the standard deviation $\sigma=0,2$.

The measure of asymmetry of a population is the skewness (also called the coefficient of skewness or asymmetry) defined on the basis of the central moment of the third order as

$$
\alpha=\frac{1}{\sigma^{3}} \int(x-\mu)^{3} \varphi(x) \mathrm{d} x
$$

Note that Figure 3.2 shows the log-normal distribution with a positive skewness $\alpha=1$.

The measure of steepness (concentration of values around the mean) is the kurtosis defined on the basis of the central moment of the fourth order as

$$
\varepsilon=\frac{1}{\sigma^{4}} \int(x-\mu)^{4} \varphi(x) \mathrm{d} x-3
$$

The kurtosis defined by equation (3.6) (with subtraction of the number 3) is zero for normal distributions (see section 3.3). Note that the kurtosis of the log-normal distribution shown in Figure 3.2 is 1,83. 
Another dimensionless parameter of a population is the coefficient of variation defined as the ratio of the standard deviation to the mean

$$
w=\frac{\sigma}{\mu}
$$

The coefficient of variation is an important measure of relative dispersion. However, it fails in the case of variables having the mean $\mu$ close to zero, then the standard deviation should be used as a measure of dispersion. Note that the coefficient of variation of common material properties (strength) may be expected to be within the interval from 0,03 to 0,30 , for actions from 0,05 (permanent load) to 1,00 (short-term variable load) and even more.

Besides the moment parameters, other parameters are also used to describe a population, e.g. the minimum and maximum values of a population $x_{\min }, x_{\max }$, the range of a population $x_{\max }-x_{\min }$, the modus $x_{\bmod }$ (defined as the value $x$, for which the probability density function $\varphi(x)$ has its maximum) and other parameters. A detailed description of these parameters can be found in the International Standards [3, 4, 5] and in specialised literature $[11,12,14]$.

\section{Example 3.2}

Parameters of the uniform distribution described in Example 3.1 may be derived using equations (3.3) to (3.7) as

$$
\mu=(a+b) / 2, \sigma=(b-a) / \sqrt{12}, \alpha=0, \varepsilon=-1.2, w=(b-a) /((a+b) \sqrt{3})
$$

The skewness and kurtosis of a uniform distribution are independent of the bounds $a$ and $b$; the skewness is zero (the distribution is symmetrical) but the kurtosis is negative -1.2 , the distribution is peaked in an opposite way from the normal distribution (values of the random variable are distributed uniformly and not concentrated around the mean as in the case of the normal distribution, see the following section 3.3). If the lower bound $a=0$ (which is sometimes assumed in practice), then

$$
\mu=0,5 b, \sigma=0,289 b, \alpha=0, \varepsilon=-1.2, w=0,577
$$

Note that the coefficient of variation $w$ in this case (when $a=0$ ) is independent of $b$ and is relatively high $(w=0,577)$.

\subsection{Sample characteristics}

A sample (also called a random sample) $x_{i}, i=1,2, \ldots n$, of a size $n$ is obtained by repeated execution of given conditions $\pi$ (the testing of a concrete cube under specified conditions). Three categories of the sample size $n$ are usually distinguished: very small samples $(n \leq 10)$, small samples $(10<n \leq 30)$ and large samples $(30<n)$. A sample is a set of units taken from a certain population (all concrete cylinders of a specified mixture), which is intended to provide information about the population. If the population is not considered (or does not exist at all), a sample is called a statistical (or numerical) sample.

The first step of analysing any sample should consist of its graphical representation by a histogram or other diagrams, examination of extreme values (outliers) and correction (withdrawal) of erroneous values. This is a very important and often hard and laborious step, which should, however, precede a further numerical treatment of the sample and an application of statistical technique for assessing the parameters of the population.

Adjusted (corrected) samples can be used for the computation of characteristics (statistics) which describe the location, dispersion, skewness, kurtosis, and other properties of 
the sample. For technical practice the most important characteristics are so-called moment characteristics, which provide the best information about the sample properties. The moment characteristics are defined analogically to the moment parameters of the population. In fact, sample characteristics are used to estimate parameters of the population. In general, however, the parameters of a population and the characteristics (statistics) assessed from a sample should be distinguished.

The important task of estimating population parameters using information obtained from a sample is a broad field of mathematical statistics, which is touched in this book only very briefly. The sample characteristics described in this section are the unbiased point estimators ("the best" point estimators) of the relevant parameters of the population. A more accurate meaning of the term "unbiased estimator" and other methods of statistical interference (e.g. interval estimators for a given confidence) are described in detail in the International Standards ISO [3, 4, 5] and in other specialised literature $[11,12,14]$.

The basic characteristic describing the sample's location, is the sample mean (also called the arithmetic mean), which is defined by the first moment about the origin

$$
m=\frac{1}{n} \sum_{i} x_{i}
$$

The sample mean $m$ is an unbiased point estimator of the population mean $\mu$ (which usually remains unknown).

The basic characteristic describing the measure of dispersion is the sample variance $s^{2}$, the definition of which is based on the second central moment

$$
s^{2}=\frac{1}{n-1} \sum_{i}\left(x_{i}-m\right)^{2}
$$

The sample variance $s^{2}$ is an unbiased point estimator of the population variance $\sigma^{2}$. The second central moment is given by the right-hand side of equation (3.9) if the denominator $n-1$ is replaced by $n$ (the denominator $n-1$ follows from the requirement of unbiased estimation of the variance $\sigma^{2}$ ). The square root of the variance $\sqrt{s^{2}}=s$ denotes the sample standard deviation. Note that the sample standard deviation $s$ is not an unbiased estimator of the population's standard deviation $\sigma[11]$.

The sample skewness $a$ (also called the coefficient of skewness or asymmetry) is a dimensionless quantity which describes the asymmetry of the sample. The unbiased point estimator of the population's skewness $\alpha$ is defined on the basis of the third central moment as

$$
a=\frac{n}{(n-1)(n-2) s^{3}} \sum_{i}\left(x_{i}-m\right)^{3}
$$

Note that the fraction on the right-hand side of relation (3.10) follows from the requirement of an unbiased estimator of the population's skewness $\alpha$.

It should be emphasised that the skewness $a$ is very sensitive to extreme values of a sample (to extreme deviations $x_{i}-m$ ) and may easily be affected by gross errors (outliers). In any case, a large sample $(n>30)$ should be used preferably for estimating the skewness. If a dubious value is obtained (in particular a negative value or a value $|a|>1$ ), it is strongly recommended to verify the outliers and to eliminate possible erroneous values and outliers.

The sample kurtosis $e$ (also the coefficient of kurtosis) is a dimensionless quantity characterising the peakness of the sample (the concentration of sample values around the 
average). The definition of the unbiased point estimator of the population kurtosis $\varepsilon$ is based on the fourth central moment

$$
e=\frac{n(n+1)}{(n-1)(n-2)(n-3))_{S}{ }^{4}} \sum_{i}\left(x_{i}-m\right)^{4}-3 \frac{(n-1)^{2}}{(n-2)(n-3)}
$$

The complicated fractions on the right-hand side of equation (3.11) follow from the requirement of the unbiased estimator of the population kurtosis $\varepsilon$. The sample kurtosis defined by equation (3.11) may be significantly affected by erroneous sample values and that is why it is rarely used in practice.

In technical practice, particularly in the construction industry, another dimensionless characteristic is used as a measure of relative dispersion. It is the ratio of the sample standard deviation $s$ to the mean $m$, called the sample coefficient of variation

$$
v=\frac{s}{m}
$$

Obviously the sample coefficient of variation $v$ defined by equation (3.12) is an analogous quantity to the coefficient of variation of a population $w$ defined similarly by equation (3.7).

\subsection{Normal distribution}

From a theoretical and practical point of view the most important type of distribution of a continuous random variable is the normal (Laplace-Gauss) distribution. A symmetric normal distribution of a variable $X$ is defined on an unlimited interval $-\infty<x<\infty$ (which can be undesirable in some practical applications) and depends on two parameters only - on the mean $\mu$ and standard deviation $\sigma$. Symbolically it is often denoted as $\mathrm{N}(\mu, \sigma)$. This distribution is frequently used as a theoretical model of various types of random variables describing some loads (self-weight), mechanical properties (strengths), and geometrical properties (outer dimensions). It is convenient for a symmetric random variable with a relatively low variance (a coefficient of variation $w<0,3$ ). It may fail for asymmetric variables with great variance and a skewness $\alpha>0,5$.

The probability density function of a normal random variable $X$ with the mean $\mu_{X}$ and standard deviation $\sigma_{X}$ is given by the exponential expression

$$
\varphi(x)=\frac{1}{\sigma_{X} \sqrt{2 \pi}} \exp \left[-\frac{1}{2}\left(\frac{x-\mu_{X}}{\sigma_{X}}\right)^{2}\right]
$$

The skewness and kurtosis defined by equations (3.5) and (3.6) are zero for any normal distribution.

No analytical expression is available for the distribution function $\Phi(x)$. Nevertheless, numerical tables are commonly available $[11,12,14]$ for the probability density function as well as for the distribution function. These tables give the probability density function $\varphi(u)$ and the distribution function $\Phi(u)$ of the standardized variable $U$ that is derived from the actual variable $X$ using the formula (applicable for any distribution)

$$
U=\frac{X-\mu_{X}}{\sigma_{X}}
$$


Here $\mu_{X}$ and $\sigma_{X}$ denote the mean and standard deviation of the actual variable $X$. The standardized random variable $U$ has a zero mean and a standard deviation equal to one; the normal standardized distribution is often symbolically denoted as $\mathrm{N}(0,1)$.

The probability density function of the standardized random variable $U$ having a normal distribution follows from equation $(3.13)$ and $(3,14)$ as

$$
\varphi(u)=\frac{1}{\sqrt{2 \pi}} \exp \left(-\frac{u^{2}}{2}\right)
$$

The probability density function of a normal and a log-normal distribution (described in the next section 3.4) having a coefficient of skewness $\alpha=1,0$ for the standardized random variable $U$ is shown in Figure 3.3. Note that the probability density function of the standardized normal distribution is plotted for $u$ within the interval $\langle-3,+3\rangle$ that covers a high occurrence probability $(0,9973)$ of the variable $U$ (in technical practice such an interval of the actual variable is sometimes denoted as $\pm 3 \sigma$ interval).

\subsection{Log-normal distribution}

A general log-normal distribution is defined on a one-sided limited interval $x_{0}<x<\infty$ or $-\infty<x<x_{0}$. It is an asymmetric distribution that partly eliminates one of the undesirable properties of the normal distribution, i.e. the infinite definition domain. A general log-normal distribution is dependent on three parameters and for that reason is often called the threeparameter log-normal distribution. Commonly, the moment parameters can be applied to define the distribution: the mean $\mu_{X}$, the standard deviation $\sigma_{X}$ and the skewness $\alpha_{X}$. Instead of the skewness $\alpha_{X}$ (when it is unknown or uncertain), the lower or upper bounds $x_{0}$ may be used.

Probability density $\varphi(u)$

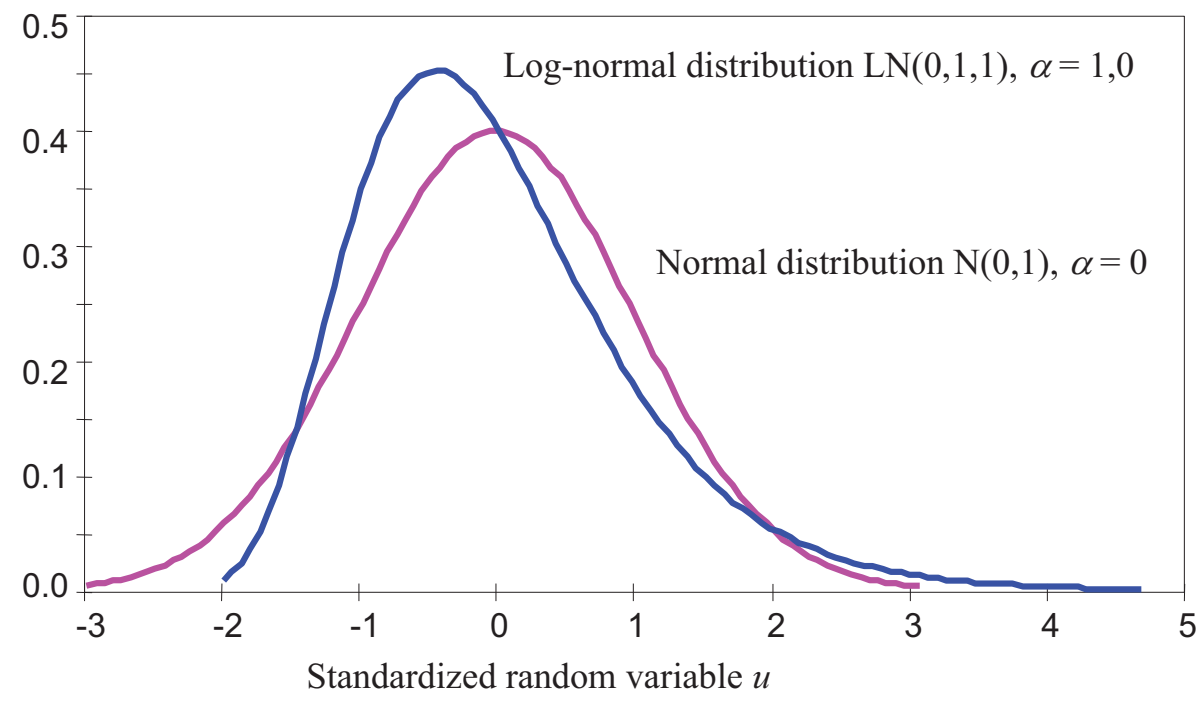

Figure 3.3. Normal and log-normal distribution (skewness $\alpha=1,0$ ) 
A random variable $X$ has a log-normal (general three-parameter) distribution if the transformed random variable

$$
Y=\ln \left|X-x_{0}\right|
$$

has a normal distribution. In this relation $x_{0}$ denotes the lower or upper bound of the variable $X$, which depends on the skewness $\alpha_{X}$. If the variable has a mean $\mu_{X}$ and standard deviation $\sigma_{X}$, then the lower or upper bound can be expressed as

$$
x_{0}=\mu_{X}-\sigma_{X} / c
$$

where the coefficient $c$ is given by the value of skewness $\alpha_{X}$ according to the relation

$$
\alpha_{X}=c^{3}+3 c
$$

from which follows an explicit relation for $c$

$$
c=\left[\left(\sqrt{\alpha_{X}^{2}+4}+\alpha_{X}\right)^{1 / 3}-\left(\sqrt{\alpha_{X}^{2}+4}-\alpha_{X}\right)^{1 / 3}\right] 2^{-1 / 3}
$$

The dependence of the limit $x_{0}$ on the coefficient $\alpha$ is apparent from Table 3.1, where the lower bound $u_{0}=-1 / \mathrm{c}$ of the standardized random variable $U=\left(X-\mu_{X}\right) / \sigma_{X}$ is given for selected values of the coefficient of skewness $\alpha_{X} \geq 0$. For $\alpha_{X} \leq 0$ values of $u_{0}$ with the inverse sign (i.e. positive) are considered. A log-normal distribution with the skewness $\alpha_{X}=0$ becomes a normal distribution $\left(u_{0}=-1 / \mathrm{c} \rightarrow \pm \infty\right)$.

Table 3.1. The coefficient $u_{0}$ for selected values of the coefficient of skewness $\alpha_{X} \geq 0$.

\begin{tabular}{|l|c|c|c|c|c|}
\hline$\alpha_{X}$ & 0 & 0,5 & 1,0 & 1,5 & 2,0 \\
\hline$u_{0}=-1 / c$ & $-\infty$ & $-6,05$ & $-3,10$ & $-2,14$ & $-1,68$ \\
\hline
\end{tabular}

When specifying a theoretical model, it is therefore possible to consider the skewness $\alpha_{X}$ or alternatively the lower or upper bound of the distribution $x_{0}$ (besides the mean $\mu_{X}$ and standard deviation $\sigma_{X}$ ). Generally, the former possibility is preferable because more credible information is usually available about the coefficient of skewness than about the lower or upper bound. In general, the skewness provides better characteristic of the overall distribution of the population (particularly of large populations) than the lower or upper bounds.

The probability density function and distribution function of the general threeparameter log-normal distribution may be obtained from the well known normal distribution using a modified (transformed) standardized variable $u$ ' obtained from the original standardized random variable $u=\left(x-\mu_{X}\right) / \sigma_{X}$ as

$$
u^{\prime}=\frac{\ln \left(\left|u+\frac{1}{c}\right|\right)+\ln \left(|c| \sqrt{1+c^{2}}\right)}{\sqrt{\ln \left(1+c^{2}\right)}} \operatorname{sign}\left(\alpha_{X}\right)
$$

Here $\operatorname{sign}\left(\alpha_{X}\right)$ equals +1 for $\alpha_{X}>0$ and -1 for $\alpha_{X}<0$. The probability density function $\varphi_{\mathrm{LN}, U}(u)$ and the distribution function $\Phi_{\mathrm{LN}, U}(u)=\Phi_{\mathrm{LN}, X}(x)$ of the log-normal distribution are given as

$$
\varphi_{\mathrm{LN}, U}(u)=\frac{\varphi\left(u^{\prime}\right)}{\left(\left|u+\frac{1}{c}\right|\right) \sqrt{\ln \left(1+c^{2}\right)}}
$$




$$
\Phi_{\mathrm{LN}, X}(x)=\Phi_{\mathrm{LN}, U}(u)=\Phi\left(u^{\prime}\right)
$$

where $\varphi\left(u^{\prime}\right)$ and $\Phi\left(u^{\prime}\right)$ denote the probability density and distribution function of the standardized normal variable.

A special case of the three-parameter log-normal distribution is the popular log-normal distribution with the lower bound at zero $\left(x_{0}=0\right)$. This distribution depends on two parameters only - the mean $\mu_{X}$ and standard deviation $\sigma_{X}$ (a symbolic notation $\operatorname{LN}(\mu, \sigma)$ is then used). In such a case it follows from equations (3.17) that the coefficient $c$ is equal to the coefficient of variation $w_{X}$. It further follows from equation (3.18) that the skewness $\alpha_{X}$ of the log-normal distribution with the lower bound at zero is given by the coefficient of variation $w_{X}$ as

$$
\alpha_{X}=3 w_{X}+w_{X}^{3}
$$

Thus, the log-normal distribution with the lower bound at zero $\left(x_{0}=0\right)$ has always a positive skewness, which may have a relatively high value (greater than 0,5); e.g. for the coefficient of variation equal to 0,30 a coefficient of skewness $\alpha_{x}=0,927$ is obtained from equation (3.23). Applications of the log-normal distribution with the lower bound at zero $\left(x_{0}=\right.$ 0 ) can thus lead to unrealistic theoretical models (usually underestimating the occurrence of negative and overestimating the occurrence of positive deviations from the mean), particularly for higher values of the coefficient of variation $w_{X}$. Then the three-parameter log-normal distribution may be used. Although the occurrence of negative values can also be undesirable (unrealistic for most mechanical quantities), it is usually negligible from a practical point of view.

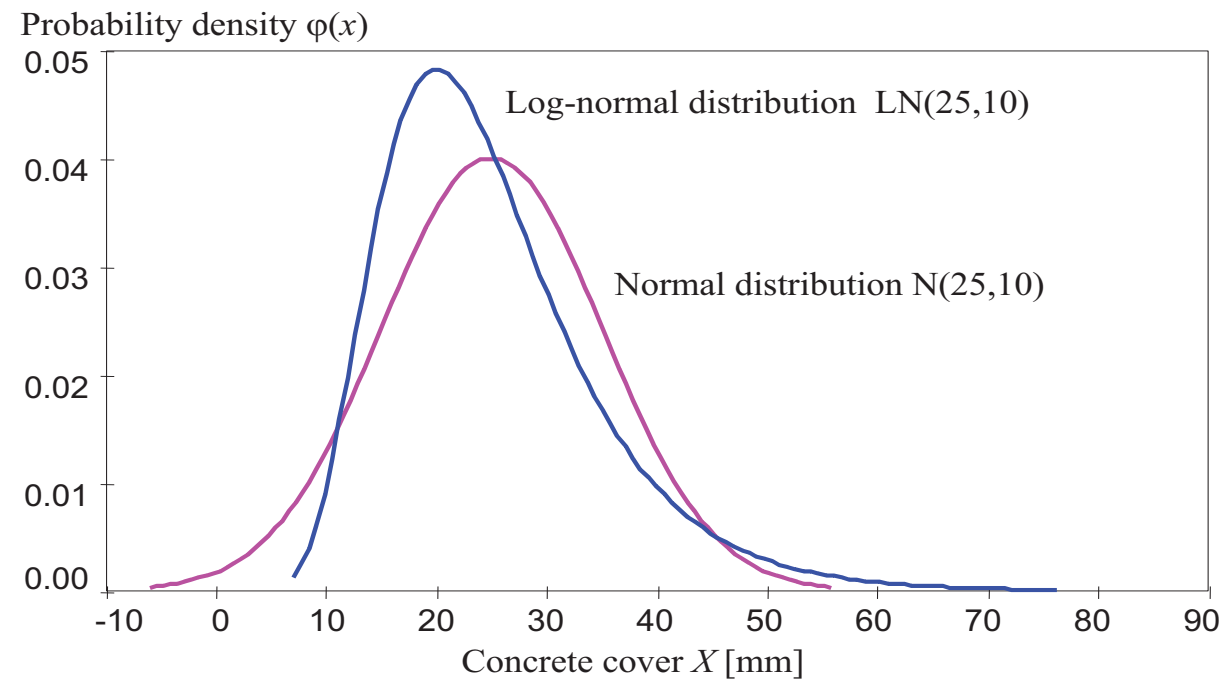

Figure 3.4. Probability density functions for the concrete cover.

\section{Example 3.3}

A concrete cover $X$ of a reinforced concrete cross-section has the mean $\mu=25 \mathrm{~mm}$ and the standard deviation $\sigma=10 \mathrm{~mm}$. The probability density function $\varphi(x)$ for a normal distribution and for a log-normal distribution with the lower bound at zero is shown in Figure 3.4 . 
It follows from Figure 3.4 that the normal distribution predicts some occurrence of negative values of the concrete cover, which may not correspond to reality. On the other hand, the log-normal distribution with the lower bound at zero may overestimate the occurrence of positive deviations, which may not be acceptable and may affect the bending resistance of the cross-section. The overestimation of the occurrence of extreme positive deviations is due to a high skewness $\alpha=1,36$ of the log-normal distribution, which follows from equation (3.23). Note that the available experimental data on a concrete cover indicate that in most cases the skewness of the distribution is less than 1 , and if no other evidence is available, then the value $\alpha \approx 0,5$ may be assumed.

The log-normal distribution is widely applied in the theory of reliability as a theoretical model for various types of random variables. In general it can be used for onesided limited asymmetric random variables including material properties, actions, and geometrical data. In particular, the log-normal distribution with the lower bound at zero $\left(x_{0}=\right.$ 0 ) is commonly used for resistance properties (strengths) of various materials (concrete, steel, timber, masonry).

\subsection{Gamma distribution}

Another popular type of one-sided limited distribution is Pearson's distribution of type III. Its detailed description is available in [11]. A special case of Pearson's distribution of type III with the lower bound at zero is the gamma distribution. The probability density function of this important distribution is dependent on two parameters only: on the mean $\mu$ and standard deviation $\sigma$. To simplify the notation two auxiliary parameters $\lambda$ and $k$ are often used

$$
\varphi(x)=\frac{\lambda^{k} x^{k-1} \exp (-\lambda x)}{\Gamma(k)}, \lambda=\frac{\mu}{\sigma^{2}}, k=\left(\frac{\mu}{\sigma}\right)^{2}
$$

where $\Gamma(k)$ is the gamma function of the parameter $k$. The moment parameters of the gamma distribution follow from equation (3.24) as

$$
\mu=\frac{k}{\lambda}, \sigma=\frac{\sqrt{k}}{\lambda}, \alpha=\frac{2}{\sqrt{k}}=\frac{2 \sigma}{\mu}=2 w, \varepsilon=\frac{3 \alpha^{2}}{2}
$$

The curve is bell shaped for $k>1$, i.e. for a skewness $\alpha<2$ (in the inverse case the gamma distribution is a decreasing function of $x$ ). For $k \rightarrow \infty$, the gamma distribution approaches the normal distribution with parameters $\mu$ and $\sigma$.

The gamma distribution is applied similarly as the log-normal distribution with the lower bound at zero. However, it differs from the log-normal distribution by its skewness, which is equal to the double of the coefficient of variation $(\underline{\alpha}=2 w)$ and is considerably lower than the skewness of the log-normal distribution with the lower bound at zero. In accordance with equation (3.23) it has the skewness $\alpha_{X}=3 w_{X}+w_{X}^{3}$. That is why the gamma distribution is more convenient for describing some geometrical quantities and variable actions that do not have a great skewness.

\section{Example 3.4}

A sample of experimental measurements of a concrete cover has the following characteristics: a sample size $n=157, m=26,8 \mathrm{~mm}, s=11,1 \mathrm{~mm}$, and $a=0,40$. It is a 
relatively large sample, which can be used for assessing skewness (long-term experience may be available to verify the obtained value). A histogram of the experimental measurements and theoretical models of the normal distribution, log-normal distribution with the origin at zero, gamma distribution and beta distribution (described in the following section) is shown in Figure 3.5. It appears that the gamma and beta distributions are the most suitable theoretical models. However, it follows from equation (3.25) that the skewness of the gamma distribution is $2 \times 11,1 / 26,3=0,83$, thus about double the value assessed from the measurements. Obviously, in this case, the beta distribution would be the most suitable theoretical model.

To choose an appropriate theoretical model for experimental data is, in general, a complicated task. Information about theoretical methods (the so-called goodness of fit tests) provided by mathematical statistics can be found in literature [11, 12, 14]. In this textbook some practical aspects and procedures only will be indicated.

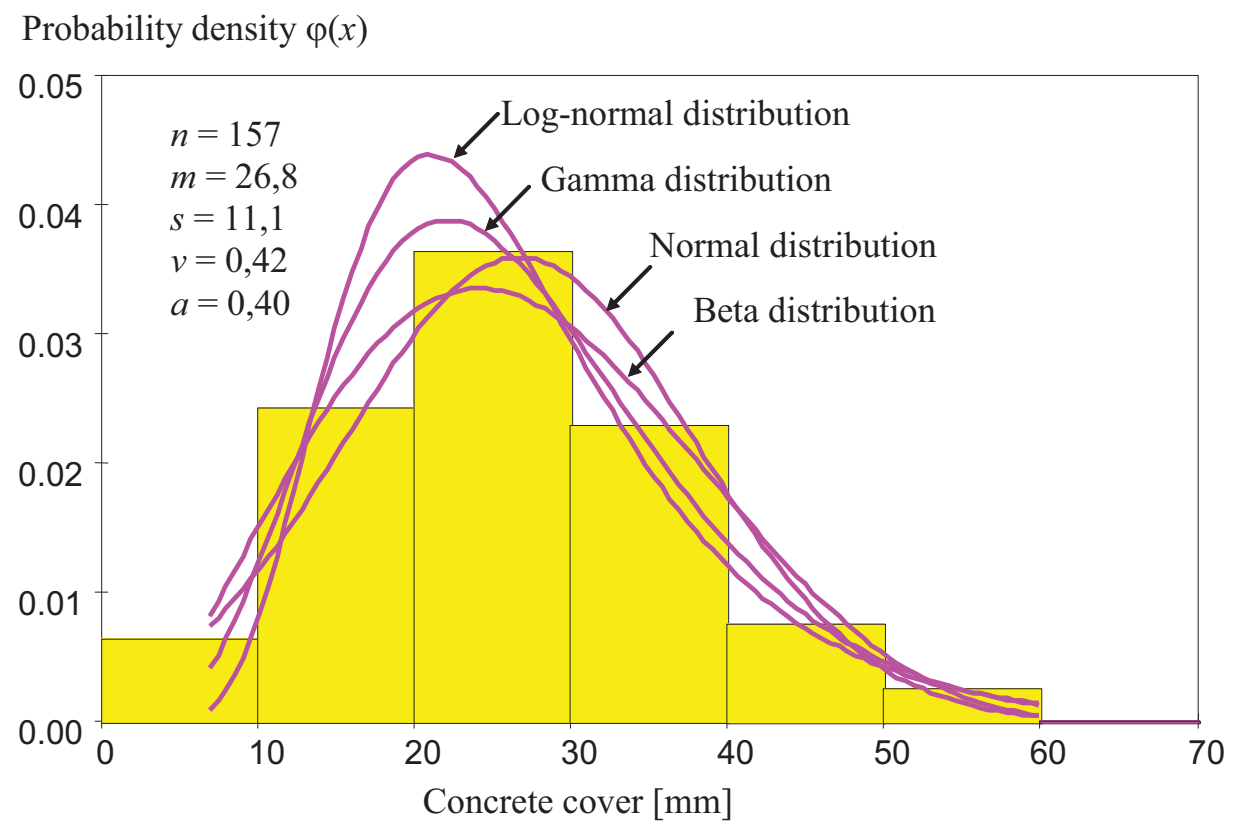

Figure 3.5. Histogram and theoretical models for concrete cover of reinforcement.

\subsection{Beta distribution}

Beta distribution (also called Pearson's distribution of type I) is defined on a two-sided interval $\langle a, b\rangle$ (this interval can be arbitrarily extended and then the distribution approaches the normal distribution). Generally, the beta distribution depends on four parameters and is used mainly in those cases where the domain of the random variable is evidently limited (some actions and geometrical data, e.g. the weight of a subway car, fire load intensity, a concrete cover of reinforcement). The principal difficulty in a practical application of the beta distribution is the necessity to estimate four parameters, for which credible data may not be always available. 
The beta distribution is usually written in the form

$$
\varphi(x)=\frac{(x-a)^{c-1}(x-b)^{d-1}}{B(c, d)(b-a)^{c+d-1}}
$$

where $c$ and $d$ are the so-called shape parameters. The lower and upper bounds are given as

$$
a=\mu-c g \sigma, b=\mu+d g \sigma, g=\sqrt{\frac{c+d+1}{c d}}
$$

where $g$ is an auxiliary parameter. The parameters $c$ and $d$ can be derived from equations (3.27) as

$$
c=\frac{\mu-a}{b-a}\left(\frac{(\mu-a)(b-\mu)}{\sigma^{2}}-1\right), d=\frac{b-\mu}{b-a}\left(\frac{(\mu-a)(b-\mu)}{\sigma^{2}}-1\right)
$$

The moment parameters of the beta distribution can be expressed in terms of the parameters $a, b, c$ and $d$ as

$$
\begin{gathered}
\mu=\frac{a+(b-a) c}{(c+d)}, \sigma=\frac{(b-a)}{(c g+d g)} \\
\alpha=\frac{2 g(d-c)}{(c+d+2)}, \varepsilon=\frac{3 g^{2}\left(2(c+d)^{2}+c d(c+d-6)\right)}{(c+d+2)(c+d+3)}-3
\end{gathered}
$$

Note that the skewness $\alpha$ and kurtosis $\varepsilon$ are dependent on only the parameters $c$ and $d$ (they are independent of the limits $a$ and $b$ ). That is why the parameters $c$ and $d$ are called the shape parameters. In practical applications the distribution is used for $c>1$ and $d>1$ (otherwise the curve is $\mathrm{J}$ or $\mathrm{U}$ shaped), for $c=d=1$ it becomes a uniform distribution, for $c=$ $d=2$ it is the so-called parabolic distribution on the interval $\langle a, b\rangle$. When $c=d$, the curve is symmetric around the mean. When $d \rightarrow \infty$, the curve becomes the type III Pearson's distribution (see section 3.5). If $c=d \rightarrow \infty$, it approaches the normal distribution. Depending on the shape parameters $c$ and $d$ the beta distribution thus covers various special types of distributions. The location of the distribution is given by the parameters $a$ and $b$.

The beta distribution can be defined in various ways. If all the four parameters $a, b, c$ and $d$ are given, it is possible to assess the moment parameters $\mu, \sigma, \alpha$ and $\varepsilon$ from equations (3.27) to (3.30). In practical applications, however, two other combinations of input parameters are likely to be applied:

1. The input parameters are $\mu, \sigma, a$ and $b$. The remaining parameters $c$ and $d$ will be assessed from equations (3.27) and (3.28), the moment parameters $\alpha$ and $\varepsilon$ from equations (3.29) and (3.30).

2. The input parameters are $\mu, \sigma, \alpha$ and one of the limits $a$ (for $\alpha>0$ ) or $b$ (for $\alpha<0$ ). The remaining parameters of the distribution $b$ (or $a$ ), $c$ and $d$ will be assessed by means of equations (3.27) to (3.29).

The beta distribution with the lower bound $a=0$ is often used in practical applications. It can be shown that in such a case the beta distribution is defined if

$$
\alpha \leq 2 w
$$

where $w=\sigma / \mu$ is the coefficient of variation. For $\alpha=2 w$ the curve becomes the type III Pearson's distribution (see section 3.5). Therefore, if the input parameters are the mean $\mu$, standard deviation $\sigma$ and a skewness $\alpha \leq 2 w$, the beta distribution with a lower limit at zero 
$(a=0)$ is fully described. The upper limit of the beta distribution with the lower bound at zero follows from the relation (3.27)

$$
b=\frac{\mu(c+d)}{c}=\frac{\mu(1+w(2+\alpha w))}{(2 w-\alpha)}
$$

In equation (3.32) the parameters $c$ and $d$ are given as

$$
\begin{gathered}
c=-\frac{\alpha}{2 w} \frac{(2 w-\alpha)^{2}-\left(4+\alpha^{2}\right)}{(w \alpha+2)^{2}-\left(4+\alpha^{2}\right)} \\
d=\frac{\alpha}{2} \frac{(2 w-\alpha)^{2}-\left(4+\alpha^{2}\right)}{(w \alpha+2)^{2}-\left(4+\alpha^{2}\right)} \frac{2+\alpha w}{\alpha-2 w}
\end{gathered}
$$

Equations (3.33) and (3.34) follow from general equations (3.25) to (3.29) for the lower bound $a=0$.

\section{Example 3.5}

Given a mean $\mu=25 \mathrm{~mm}$, a standard deviation $10 \mathrm{~mm}(w=0,40)$, and a skewness $\alpha=0,5$, assess the parameters of a beta distribution with the origin at zero $(a=0)$ for a reinforcement cover layer. Equation 3.31 is satisfied $(0,5<2 \times 0,4)$. From equations (3.33) and (3.34) it follows that

$$
\begin{gathered}
c=-\frac{0,5}{2 \times 0,4} \frac{(2 \times 0,4-0,5)^{2}-\left(4+0,5^{2}\right)}{(0,4 \times 0,5+2)^{2}-\left(4+0,5^{2}\right)}=4,406 \\
d=\frac{0,5}{2} \frac{(2 \times 0,4-0,5)^{2}-\left(4+0,5^{2}\right)}{(0,4 \times 0,5+2)^{2}-\left(4+0,5^{2}\right)} \frac{2+0,5 \times 0,4}{0,5-2 \times 0,4}=12,926
\end{gathered}
$$

For the upper bound of the distribution it follows from equation (3.32) that

$$
b=\frac{25(4,407+12,926)}{4,407}=98,326
$$

Figure 3.6 shows the beta distribution having the parameters assessed above together with corresponding normal, log-normal and Gamma distribution that have the same mean $\mu$ and standard deviation $\sigma$. Obviously, there are considerable differences between the distributions indicated in Figure 3.6.

The normal distribution (skewness $\alpha=0$ ) predicts the occurrence of negative values, which may not comply with the real conditions for the reinforcement cover layer. The lognormal distribution with the lower bound at zero has a skewness $\alpha=1,264$ (given by equation (3.23)), which does not correspond to experimental results and leads to an overestimation of the occurrence of positive deviations (which may further lead to unfavourable consequences for the resistance of the reinforced concrete element). The gamma distribution has a skewness $\alpha=2 w=0,8$ (equation (3.25)) and is closer to the experimental value 0,5 . The most convenient model seems to be the beta distribution having a skewness $\alpha=0,5$ obtained from experimental data.

The above discussion can be supplemented by statistical tests $[11,12,14]$. On the other hand it should be mentioned that goodness of fit tests often fail and do not lead to an 
unambiguous conclusion. In such a case the selection of a convenient model depends on the character of the basic variable, on available experience and on common experience.

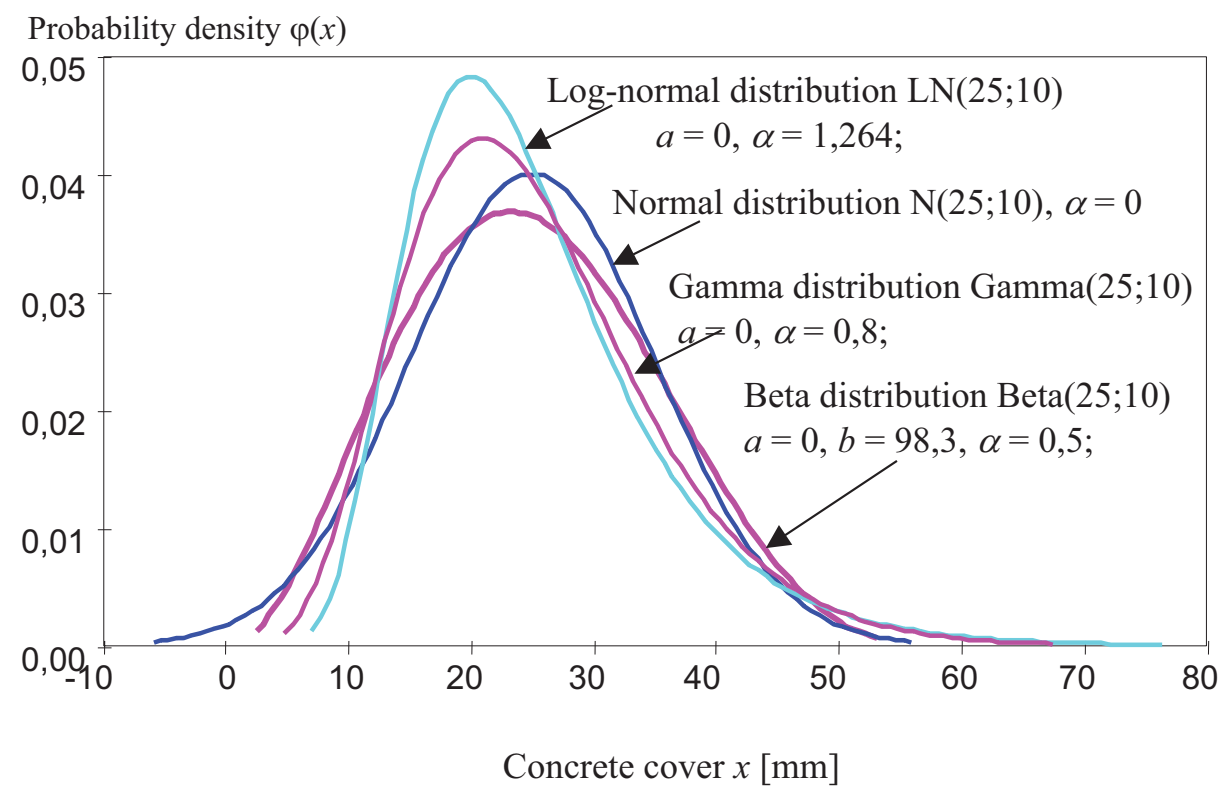

Figure 3.6. Normal, Log-normal, Gamma and Beta distributions for the concrete cover layer of reinforcement in a reinforced concrete element

\subsection{Gumbel and other distributions of extreme values}

The extreme values (maximal or minimal) in a population of a certain size are random variables and their distribution is very important for the theory of structural reliability. Three types of the distribution of extreme values are usually covered in specialised literature, they are denoted as types I, II and III. Each of the types has two versions - one for the distribution of minimal values, the second for the distribution of maximal values. All these types of distribution have a simple exponential shape and are convenient to work with. The extreme value distribution of type I, which is commonly called the Gumbel distribution, is described in detail. Descriptions of the other types of distribution can be found in specialised literature [11].

The distribution function of type I for the version maximal values distribution (Gumbel distribution of maximum values) has the form

$$
\Phi(x)=\exp \left(-\exp \left(-c\left(x-x_{\text {mod }}\right)\right)\right)
$$

It is a distribution defined on an infinite interval, which depends on two parameters: on the mode $x_{\text {mod }}$ and parameter $c>0$. By differentiating the distribution function we obtain the probability density function in the form

$$
\varphi(x)=c \exp \left(-c\left(x-x_{\bmod }\right)-\exp \left(-c\left(x-x_{\bmod }\right)\right)\right)
$$

Both these parameters can be assessed from the mean $\mu$ and standard deviation $\sigma$

$$
x_{\text {mod }}=\mu-0,577 \sigma \frac{\sqrt{6}}{\pi}
$$




$$
c=\frac{\pi}{\sigma \sqrt{6}}
$$

The skewness and kurtosis of the distribution are constant: $\alpha=1,14, \varepsilon=2,4$.

An important feature of the Gumbel distribution is an easy transformation of the distribution function $\Phi(x)$ of an original random variable having the mean $\mu$ and standard deviation $\sigma$ to the distribution function $\Phi_{N}(x)$ for the maxima of populations that are $N$ times greater than the original population. If individual original populations constituting a new $N$ times greater population are mutually independent, then the distribution function $\Phi_{N}(x)$ is given as

$$
\Phi_{N}(x)=(\Phi(x))^{N}
$$

By substitution of equation (3.35) into equation (3.39) $\Phi_{N}(x)$ can be written as

$$
\Phi_{N}(x)=\exp \left(-\exp \left(-c\left(x-x_{\bmod }-\ln N / c\right)\right)\right)
$$

It follows from equations (3.35) and (3.40) that the mean $\mu_{N}$ and standard deviation $\sigma_{N}$ of the maxima of the new $N$ times greater population are

$$
\mu_{N}=\mu+\ln (N / c)=\mu+0,78 \ln (N \sigma), \sigma_{N}=\sigma
$$

Thus the standard deviation of the original population does not change and $\sigma_{N}=\sigma$, but the mean $\mu_{N}$ is greater than the original value $\mu$ by $\ln (N / c)$.

\section{Example 3.6}

One-year maxima of wind pressure are described by a Gumbel distribution with a mean $\mu_{1}=0,35 \mathrm{kN} / \mathrm{m}^{2}, \sigma_{1}=0,06 \mathrm{kN} / \mathrm{m}^{2}$. The corresponding parameters of 50 -year maximum value distribution, i.e. parameters $\mu_{50}$ and $\sigma_{50}$, follow from equation (3.41)

$$
\mu_{50}=0,35+0,78 \times \ln (50 \times 0,06)=0,53 \mathrm{kN} / \mathrm{m}^{2}, \sigma_{50}=0,06 \mathrm{kN} / \mathrm{m}^{2}
$$

Figure 3.7 shows both the distributions of one-year and fifty-year maxima of wind pressure described by a Gumbel distribution.

Probability density $\varphi_{N}(x)$

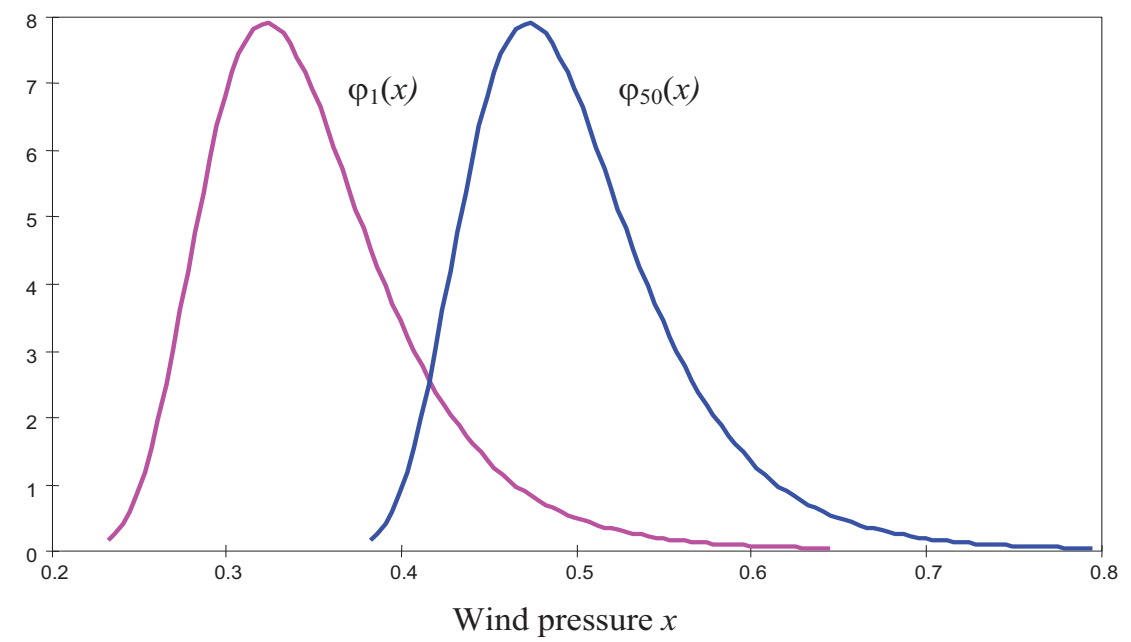

Figure 3.7. Distribution of maximum wind pressure over the periods of 1 year and 50 years. 
The distribution function of type I, for the minimal values distribution (Gumbel distribution of minimum values) has the form

$$
\Phi(x)=1-\exp \left(-\exp \left(-c\left(x_{\bmod }-x\right)\right)\right)
$$

This distribution is symmetric to the distribution of maximal values given by equation (3.35). It is therefore also defined on an open interval and depends on two parameters: on the mode $x_{\bmod }$ and parameter $c>0$. By differentiating the distribution function we obtain the probability density function in the form

$$
\varphi(x)=c \exp \left(-c\left(x_{\bmod }-x\right)-\exp \left(-c\left(x_{\bmod }-x\right)\right)\right)
$$

Both these parameters can be assessed from the mean $\mu$ and standard deviation $\sigma$

$$
\begin{gathered}
x_{\text {mod }}=\mu+0,577 \sigma \frac{\sqrt{6}}{\pi} \\
c=\frac{\pi}{\sigma \sqrt{6}}
\end{gathered}
$$

The probability density functions of the minimum values are symmetric to the shape of maximal values relative to the mode $x_{\bmod }$, as is apparent from Figure 3.8.

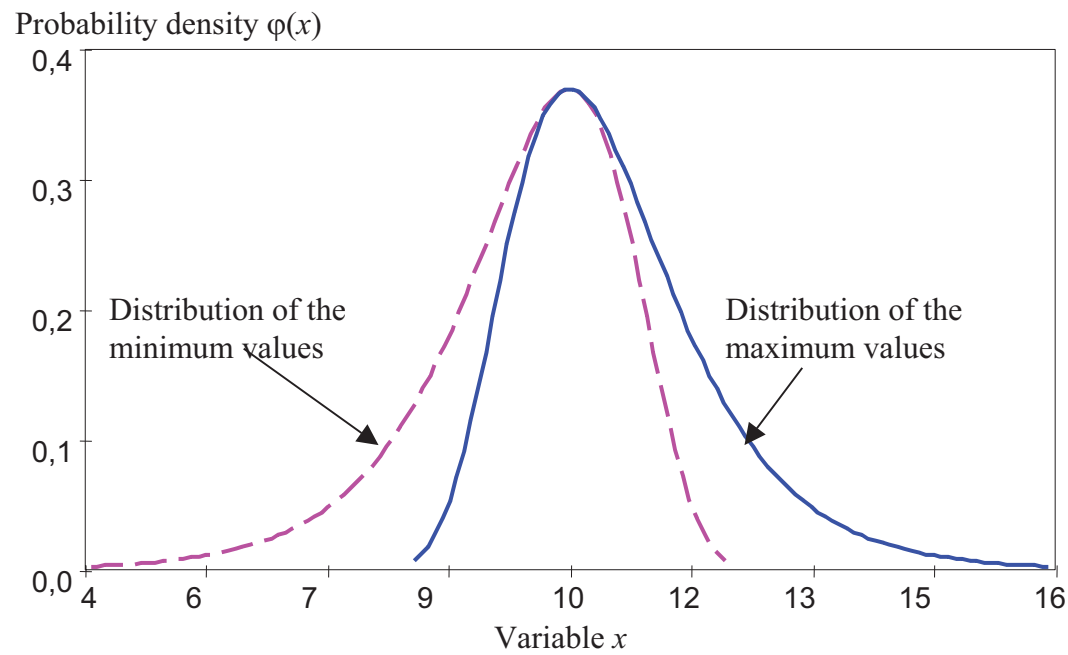

Figure 3.8. The Gumbel distribution of the minimum and maximum values.

In a similar way, the type II distribution, the so-called Fréchet distribution, and type III distribution, the so-called Weibull distribution, are defined. All three types of distribution complement each other with regard to possible values of the skewness $\alpha$. Each type covers a certain area of skewness, as shown in Figure 3.9.

Types I and II of the extreme values distribution are often applied in the description of quantities of which the maximal values are studied (actions), and the type III distribution is applied for quantities of which the minimal values are studied (e.g. strength and other material properties). 
Distribution of the maximum values

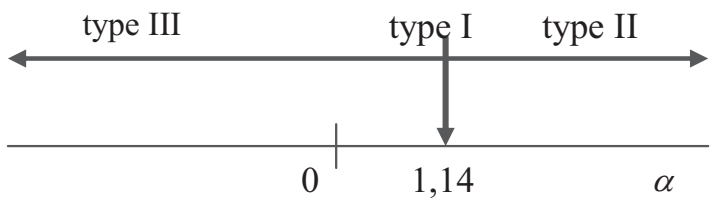

Distribution of the minimum values

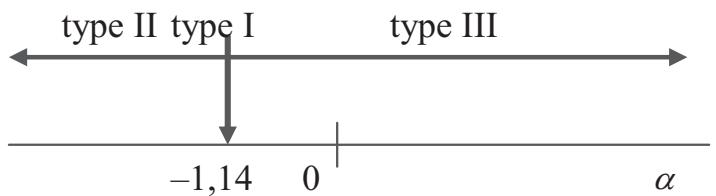

Figure 3.9. Types of the distribution of extreme values versus the skewness $\alpha$.

\subsection{Multivariate random variables}

This section contains remarks and basic information on multivariate, particularly on bivariate, random variables. A detailed discussion concerning the multivariate random variables, probabilistic models, parameters of population and sample characteristics can be found in literature [11, 12].

If two variables (two characteristics) $X$ and $Y$ are studied for each item (entity), every time a set of conditions $\pi$ (see section 2.1) is realised, i.e. a certain random event is realised, and given that the variable $X$ takes on the very value $x$ and the variable $Y$ takes on the very value $y$, then the variables $X$ and $Y$ form a pair of joint random variables. An example is the force $X$ and the weight $Y$ studied when a concrete cube fails when loaded under given conditions in a test machine (see Example 2.2). It is certainly possible to study more than two characteristics, e.g. the force, weight and moisture content. In a general case variables $X_{1}$, $X_{2}, \ldots X_{\mathrm{n}}$ are studied, which we will denote for simplicity as a vector $\boldsymbol{X}\left[X_{1}, X_{2}, \ldots X_{\mathrm{n}}\right]$ and its realizations $x_{1}, x_{2}, \ldots x_{\mathrm{n}}$ as a vector $\boldsymbol{x}\left[x_{1}, x_{2}, \ldots x_{\mathrm{n}}\right]$. The following summary is particularly concerned with two-dimensional random variables, which have two components (two joint random variables) $X$ and $Y$.

The summary of all possible realizations $x$ and $y$ of a pair of joint random variables $X$ and $Y$ is called the two-dimensional population. A pair of joint random variables $X$ and $Y$ is also called the two-dimensional random variable. Similarly to the one-dimensional random variable the two-dimensional random variable is described by the distribution of probabilities, i.e. by a function which determines the probability that the random variables $X$ and $Y$ make up part of some given sets (for continuous random variables) or take on some given values (for discrete random variables). The two-dimensional distribution function $\Phi(x, y)$ (sometimes denoted $\left.\Phi_{X Y}(x, y)\right)$ gives, for every pair of values $x, y$, the probability that the random variable $X$ is less than or equal to $x$, and the random variable $Y$ is less than or equal to $y$

$$
\Phi(x, y)=\mathrm{P}(X \leq x ; Y \leq y)
$$

The probability density function of a continuous random variable $\varphi(x)$ is the derivative (if it exists) of the distribution function 


$$
\varphi(x, y)=\frac{\partial^{2} \Phi(x, y)}{\partial x \partial y}
$$

The marginal distribution function of the variable $X, \Phi_{X}(x)$, is a special case of the distribution function $\Phi(x, y)$ without any constraint on the variable $Y$, i.e. for all the realizations $Y<\infty$

$$
\Phi(x, \infty)=\mathrm{P}(X \leq x ; Y \leq \infty)=\Phi_{X}(x)
$$

The marginal distribution function of the variable $Y, \Phi_{Y}(y)$, is defined in a similar way. It is a special case of the distribution function $\Phi(x, y)$ without any constraint on the variable $X$, i.e. for the sum of all possible realizations of the variable $X<\infty$

$$
\Phi(\infty, y)=\mathrm{P}(X \leq \infty ; Y \leq y)=\Phi_{Y}(y)
$$

We say that the random variables $X$ and $Y$ are independent if it holds that

$$
\Phi(x, y)=\Phi(x, \infty) \Phi(\infty ; y)=\Phi_{X}(x) \Phi_{Y}(y)
$$

Then it holds for the probability density function that

$$
\varphi(x, y)=\varphi_{X}(x) \varphi_{Y}(y)
$$

where $\varphi_{X}(x)$ and $\varphi_{Y}(y)$ are the marginal probability density functions of the variables $X$ and $Y$.

The two-dimensional random variable is described by moment parameters and various types of distribution (usually by the normal), similarly to one-dimensional variables. Besides the one-dimensional moments which lead to the definition of averages $\mu_{X}, \mu_{Y}$, and the standard deviations $\sigma_{X}, \sigma_{Y}$, the joint moments of both variables $X$ and $Y$ are also applied. The most important one is the joint central moment of the first order $\sigma_{X Y}$, which is called the covariance

$$
\sigma_{X Y}=\int \varphi(x, y)\left(x-\mu_{X}\right)\left(y-\mu_{Y}\right) d x d y
$$

The covariance provides the basis for the definition of the correlation coefficient $\rho_{X Y}$

$$
\rho_{X Y}=\frac{\sigma_{X Y}}{\sigma_{X} \sigma_{Y}}
$$

It always holds for the value of the correlation coefficient that $-1 \leq \rho_{X Y} \leq+1$. If the variables $X$ and $Y$ are independent, then $\rho_{X Y}=0$. An inverse proposition holds only in the case of the two-dimensional normal distribution (which is described below). In the case of multivariate random variables $\boldsymbol{X}\left[X_{1}, X_{2}, \ldots X_{\mathrm{n}}\right]$, the covariance $\sigma_{i j}$ and the correlation coefficients $\rho_{i j}$ between the individual components $X_{1}, X_{2}, \ldots X_{\mathrm{n}}$ form matrices. The matrix of covariances is applied in the transformation of the vector of dependent variables to the vector of independent random variables, which are used in reliability analysis of more complex problems (see the software product STRUREL [24]).

Using a sample of pair observations $x_{1}, y_{1} ; x_{2}, y_{2} ; \ldots ; x_{n}, y_{n}$, the sample covariance is assessed

$$
s_{X Y}=\frac{1}{n-1} \sum_{i}\left(x_{i}-m_{X}\right)\left(y_{i}-m_{y}\right)
$$

which is an unbiased estimator of the population's covariance (the denominator $n-1$ follows from the requirement that the estimator is unbiased, similarly to the case of the sample variance (3.9)). Analogically to (3.53) it therefore holds for the sample correlation coefficient that 


$$
r_{X Y}=\frac{s_{X Y}}{s_{X} s_{Y}}=\frac{\sum_{i}\left(x_{i}-m_{X}\right)\left(y_{i}-m_{Y}\right)}{\sqrt{\sum_{i}\left(x_{i}-m_{X}\right)^{2} \sum_{i}\left(y_{i}-m_{Y}\right)^{2}}}
$$

After substitution of relations (3.54) and (3.9) their denominators $n-1$ do not apply in this formula.

The sample correlation coefficient $r_{X Y}$ is often used for the numerical expression of the mutual linear dependence between $X$ and $Y$ in a number of pair observations. The value of $r_{X Y}$ lies between -1 and +1 . If it equals one of these bounds, it means that the dependence between $X$ and $Y$ in a number of pair observations is exactly linear. When possible, a scatter diagram showing the observed set should be used to verify the linearity, and possibly to reduce the domain so that the assumption of linearity is justified.

A two-dimensional normal distribution of two continuous random variables $X$ and $Y$ having parameters $\mu_{x}, \mu_{y}, \sigma_{x}, \sigma_{y}$, and a correlation coefficient $\rho_{x y}=\rho$ is given by the following equation (3.56)

$$
\varphi(x, y)=\frac{1}{2 \pi \sigma_{X} \sigma_{Y} \sqrt{1-\rho^{2}}} \exp \left(-\frac{1}{2\left(1-\rho^{2}\right)}\left(\left(\frac{x-\mu_{X}}{\sigma_{X}}\right)^{2}-2 \rho\left(\frac{x-\mu_{X}}{\sigma_{X}}\right)\left(\frac{y-\mu_{Y}}{\sigma_{Y}}\right)+\left(\frac{y-\mu_{Y}}{\sigma_{Y}}\right)^{2}\right)\right)
$$

The marginal distributions $\varphi_{X}(x)$ and $\varphi_{Y}(y)$ are also normal and have parameters $\mu_{X}, \sigma_{X}$ and $\mu_{Y}, \sigma_{Y}$ similarly to the conditional distributions for given $y=y_{0}$ and $x=x_{0}$, which have parameters $\mu_{x}+\rho\left(y_{0}-\mu_{Y}\right) \sigma_{X} / \sigma_{Y}, \sigma_{x}\left(1-\rho^{2}\right)^{1 / 2}$ and $\mu_{Y}+\rho\left(x_{0}-\mu_{X}\right) \sigma_{Y} / \sigma_{X}, \sigma_{Y}\left(1-\rho^{2}\right)^{1 / 2}[11]$. The conditional distributions may come in useful for (very frequent) indirect experimental verification of properties of one of the joint random variables $X$ and $Y$ by means of the other.

Similarly, as in the case of the one-dimensional random variable through transformations, the standardized random variables $U$ and $V$ are given as

$$
U=\frac{X-\mu_{X}}{\sigma_{X}}, \quad V=\frac{Y-\mu_{Y}}{\sigma_{Y}}
$$

The standardized two-dimensional normal distribution can then be written in the form

$$
\varphi(u, v)=\frac{1}{2 \pi \sqrt{1-\rho^{2}}} \exp \left(-\frac{1}{2\left(1-\rho^{2}\right)}\left(u^{2}-2 \rho u v+v^{2}\right)\right)
$$

The bivariate normal distribution can be generalized $[11,14]$ to a distribution of multivariate random variables described by the vector $\boldsymbol{X}\left[X_{1}, X_{2}, \ldots X_{n}\right]$, where the covariance's and correlation coefficients between the individual components $X_{1}, X_{2}, \ldots X_{n}$, form matrices.

\subsection{Combination of two random samples}

Sometimes it is required to combine two random samples taken from one population assuming that the characteristics of both the samples are known, but the original observations $x_{i}$ are not available. It must be emphasised that only homogeneous samples of the same origin (taken under the same conditions) should be combined. Violation of this important assumption could lead to incorrect results.

Assume that a first sample of the size $n_{1}$ has the characteristics $m_{1}, s_{1}, a_{1}$, a second sample of the size $n_{2}$ has the characteristics $m_{2}, s_{2}, a_{2}$. The characteristics of a combined sample of the size $n$ can be determined from the following expressions: 


$$
\begin{gathered}
n=n_{1}+n_{2} \\
m=\frac{n_{1} m_{1}+n_{2} m_{2}}{n} \\
s^{2}=\frac{n_{1} s_{1}^{2}+n_{2} s_{2}^{2}}{n}+\frac{n_{1} n_{2}}{n^{2}}\left(m_{1}-m_{2}\right)^{2} \\
a=\frac{1}{s^{3}}\left[\frac{n_{1} s_{1}^{3} a_{1}+n_{2} s_{2}^{3} a_{2}}{n}+\frac{3 n_{1} n_{2}\left(m_{1}-m_{2}\right)\left(s_{1}^{2}-s_{2}^{2}\right)}{n^{2}}-\frac{n_{1} n_{2}\left(n_{1}-n_{2}\right)\left(m_{1}-m_{2}\right)^{3}}{n^{2}}\right]
\end{gathered}
$$

It is interesting to note that the standard deviation $s$ is dependent not only on the standard deviations, but also on the means of both the samples. Similarly, the skewness depends also on the characteristics of lower order (means and standard deviations).

In some cases it may occur that the size of one sample, say $n_{1}$, is not known, and only the first two characteristics $m_{1}, s_{1}$ are available. This is a typical situation of updating previous data having the characteristics $m_{1}, s_{1}$, using newly observed data of the size $n_{2}$ having the characteristics $m_{2}, s_{2}$.

Following the Bayesian concept $[3,11]$, the unknown value $n_{1}$ and a corresponding degree of freedom $v_{1}$ may be assessed using the relations for the coefficients of variation of the mean and standard deviation $w(\mu)$ and $w(\sigma)$, (the parameters $\mu$ and $\sigma$ are considered as random variables in Bayes' concept) for which it holds

$$
n_{1}=\left[s_{1} /\left(m_{1} w(\mu)\right)\right]^{2}, v_{1}=1 /\left(2 w(\sigma)^{2}\right)
$$

Both unknown variables $n_{1}$ and $v_{1}$ may be assessed independently (generally $v_{1} \neq n_{1}-$ 1 ), depending on previous experience with a degree of uncertainty of the estimator of the mean $\mu$ and the standard deviation $\sigma$ of the population. Note that for a new sample it holds that $v_{2}=n_{2}-1$.

When the sample size $n_{1}$ and the degree of freedom $v_{1}$ are estimated, the degree of freedom $v$ is given as $[3,11]$

$$
v=v_{1}+v_{2}-1 \text { if } n_{1} \geq 1, v=v_{1}+v_{2} \text { if } n_{1}=0
$$

Then the resulting size of the combined sample $n$ and the mean $m$ is given by equations (3.59) and (3.60), the standard deviation $s$ is determined from a modified equation (3.61) as

$$
s^{2}=\left[v_{1} s_{1}^{2}+v_{2} s_{2}^{2}+\frac{n_{1} n_{2}}{n}\left(m_{1}-m_{2}\right)^{2}\right] / v
$$

The above relationship may be easily applied using the Mathcad sheet included in annex 4 to this textbook.

\section{Example 3.7}

Suppose that from previous production of a given type of concrete the following experience is available for its strength

$$
m_{1}=30,1 \mathrm{MPa}, w(\mu)=0,50, s_{1}=4,4 \mathrm{MPa}, w(\sigma)=0,28 .
$$

For unknown characteristics $n_{1}$ and $v_{1}$ it follows from equation (3.63) that 


$$
n_{1}=\left(\frac{4,4}{30,1} \frac{1}{0,50}\right)^{2} \approx 0, v_{1}=\frac{1}{2 \times 0,28^{2}} \approx 6
$$

Thus, the following characteristics are considered further on: $n_{1}=0$ and $v_{1}=6$.

To verify the quality of the concrete, new measurements have been carried out using specimens from the same type of concrete. The following strength characteristics have been obtained

$$
n_{2}=5, v_{2}=n_{2}-1=4, m_{2}=29,2 \mathrm{MPa}, s_{2}=4,6 \mathrm{MPa} .
$$
follows

Using equations (3.59), (3.60), (3.64) and (3.65), the updated characteristics are as

$$
\begin{gathered}
n=0+5=5 \\
v=6+4=10 \\
m=\frac{0 \times 30,1+5 \times 29,2}{5}=29,2 \mathrm{MPa} \\
s^{2}=\left[6 \times 4,4^{2}+4 \times 5,6^{2}+\frac{0 \times 5}{5}(30,1-29,2)^{2}\right] / 10=4,5^{2} \mathrm{MPa}^{2}
\end{gathered}
$$

Thus, using the previous information the standard deviation of the new measurements could be decreased from $\mathrm{s}=5,6 \mathrm{MPa}$ to $\mathrm{s}=4,5 \mathrm{MPa}$.

However, it should be noted that the combination of the previous information with the current measurements might not always lead to favourable results. For example, if the coefficients of variation are $w(\mu)=0,2$ and $w(\sigma)=0,6$, then the unknown characteristics $n_{1}$ and $v_{1}$ follow from equation (3.63) as

$$
n_{1}=\left(\frac{4,4}{30,1} \frac{1}{0,2}\right)^{2} \approx 1 ; v_{1}=\frac{1}{2 \times 0,6^{2}} \approx 1
$$

In this case

$$
\begin{gathered}
n=1+5=6 \\
v=1+4-1=4 \\
m=\frac{1 \times 30,1+5 \times 29,2}{6}=29,35 \mathrm{MPa} \\
s^{2}=\left[1 \times 4,4^{2}+4 \times 5,6^{2}+\frac{1 \times 5}{6}(30,1-29,2)^{2}\right] / 4=6,03^{2} \mathrm{MPa}^{2}
\end{gathered}
$$

In this case, the mean increased slightly from 29,2 to 29,35 , the standard deviation increased considerably from 5,6 to 6.03 . However, this is an extreme case, caused by unfavourable estimates of $n_{1}, v_{1}$ and $v$ following from equations (3.63) and (3.64). In practical applications these equations should be applied with caution, particularly in extreme cases similar to the above example. In connection with the above warning an important assumption mentioned at the beginning of this section should be stressed. Only those samples that are evidently taken from the same population can be used for combining or updating statistical data, otherwise the results of the combination of two random samples may lead to incorrect results. 


\subsection{Functions of random variables}

Structural behaviour is commonly described by a function of random variables $X, Y$, that are random variables, resulting in the variable $Z$ given by a general relationship

$$
Z=\mathrm{f}(X, Y, \ldots)
$$

$Z$ is therefore also a random variable having parameters $\mu_{Z}, \sigma_{Z}, \alpha_{Z}$, for which (using the Taylor expansion of $\mathrm{f}(X, Y, \ldots)$ into a power series) approximate relationships may be found

$$
\begin{gathered}
\mu_{Z}=\mathrm{f}_{1}\left(\mu_{X}, \mu_{Y}, \ldots \sigma_{X}, \sigma_{Y}, \ldots, \alpha_{X}, \alpha_{Y, \ldots}\right) \\
\sigma_{Z}=\mathrm{f}_{2}\left(\mu_{X}, \mu_{Y}, \ldots \sigma_{X}, \sigma_{Y}, \ldots, \alpha_{X}, \alpha_{Y, \ldots}\right) \\
\alpha_{Z}=\mathrm{f}_{3}\left(\mu_{X}, \mu_{Y}, \ldots \sigma_{X}, \sigma_{Y}, \ldots, \alpha_{X}, \alpha_{Y, \ldots}\right)
\end{gathered}
$$

Annex 3 provides relationships (3.67) to (3.69) for selected functions (3.66) considering two random variables $X$ and $Y$. These relationships may be effectively applied to simplify a number of common expressions entering the limit state functions used in reliability analysis of structural members.

\section{Example 3.8}

Consider a simple product of two random variables $X$ and $Y$. Equation (3.66) is then written as

$$
Z=X \times Y
$$

Using annex 3 the following relationships for the basic parameters may be found

$$
\begin{gathered}
\mu_{Z}=\mu_{X} \times \mu_{Y} \\
w_{Z}^{2}=w_{X}^{2}+w_{Y}^{2}+w_{X}^{2} w_{Y}^{2} \\
\alpha_{Z}=\frac{w_{X}^{3} \alpha_{X}+w_{Y}^{2} \alpha_{Y}+6 w_{X}^{2} w_{Y}^{2}}{\left(w_{X}^{2}+w_{Y}^{2}+w_{X}^{2} w_{Y}^{2}\right)^{3 / 2}}=\frac{w_{X}^{3} \alpha_{X}+w_{Y}^{2} \alpha_{Y}+6 w_{X}^{2} w_{Y}^{2}}{w_{Z}^{3}}
\end{gathered}
$$

\subsection{Updating of probability distributions}

If the prior probabilities $p^{\prime}$ are described by a continuous probability density function $\varphi(x)$ of a random variable $X$ (for details see chapter 3 ) and likelihood by conditional density function $\varphi(I \mid x)$ where $I$ denotes outcomes of additional investigation $I$, then a posteriori (updated) probability density $\varphi(x \mid I)$ may be derived from (2.25) by substituting $\varphi(x)$ for $p_{i}$ ', $\varphi(I \mid x)$ for $l_{i}, \varphi(x \mid I)$ for $p_{i}$ " and using integration instead of the summation as

$$
\varphi(x \mid I)=\frac{\varphi(x) \varphi(I \mid x)}{\int \varphi(x) \varphi(I \mid x) \mathrm{d} x}
$$

Note that the likelihood $\varphi(I \mid x)$ is the conditional probability density function describing the probability that the outcome of the updating investigation $I$ (information obtained from $I$ ) is due to the occurrence of $x$. Formulae (3.70) can be used for the updating of distribution functions when additional experimental investigations are used for assessing new or existing structures. 


\section{Example 3.9}

Assume that a variable $X$ has a normal prior distribution with probability density function $\varphi(x)$ having the mean $\mu$ and the standard deviation $\sigma$. Additional investigation indicated that the likelihood function $\varphi(I \mid x)$ is described by a general three-parameter lognormal distribution having the same standard deviation $\sigma$ but the mean equal to $\mu+0,5 \sigma$ and the skewness $\alpha=1$. Using a numerical integration of it follows from formulae (3.66) that the updated distribution $\varphi(x \mid I)$ has the following parameters

$$
\begin{gathered}
\mu_{x \mid I}=\mu+0,18 \sigma \\
\sigma_{x \mid I}=0,64 \sigma \\
\alpha_{x \mid I}=0,39
\end{gathered}
$$

Figure 3.10 shows the prior probability density $\varphi(u)$, likelihood $\varphi(I \mid u)$ and the updated probability density function $\varphi(u \mid I)$ using standardized random variable $U$.

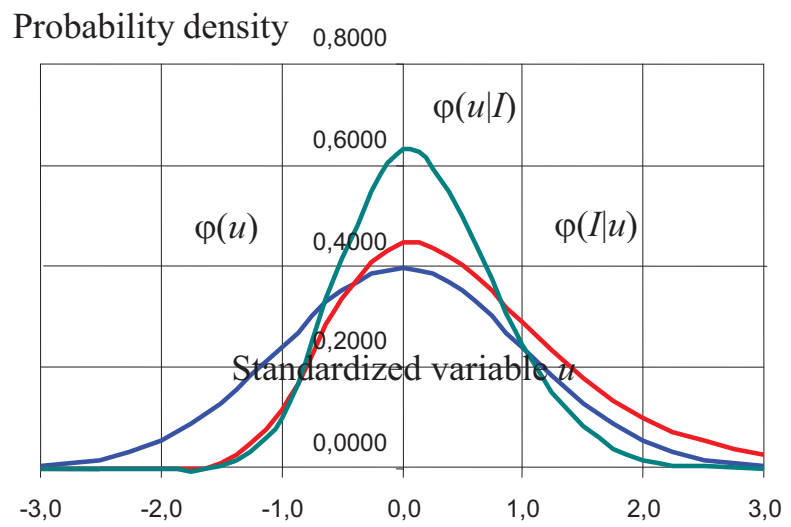

Figure 3.10. Prior probability density $\varphi(u)$, likelihood $\varphi(I \mid u)$ and the updated probability density function $\varphi(u \mid I)$.

It follows from Figure 3.10 that the updated distribution has considerably lower variability than the prior distribution. Obviously updating of probability distributions may be extremely effective when assessing characteristic values of the resistance variables using additional tests. 


\subsection{Fractile of theoretical models}

One of the most important keywords of the theory of structural reliability is the term fractile of a random variable $X$ (or of its probability distribution), sometimes also called the quantile. For a given probability $p$, the $p$-fractile $x_{p}$ denotes such a value of the random variable $X$, for which it holds that values less than or equal to $x_{p}$ occur with the probability $p$. If $\Phi(x)$ is the distribution function of the random variable $X$, then it follows from equation (3.1) that the value $\Phi\left(x_{p}\right)$ is equal to the probability $p$, thus the fractile $x_{p}$ can be defined as

$$
\mathrm{P}\left(X \leq x_{p}\right)=\Phi\left(x_{p}\right)=p
$$

The same definition holds for a standardised random variable $U$ (given by the transformation equation (3.14)), when in equation (4.1) $U$ is substituted for $X$ and $u_{p}$ is substituted for $x_{p}$. Figure 4.1 illustrates the definition given by equation (4.1).

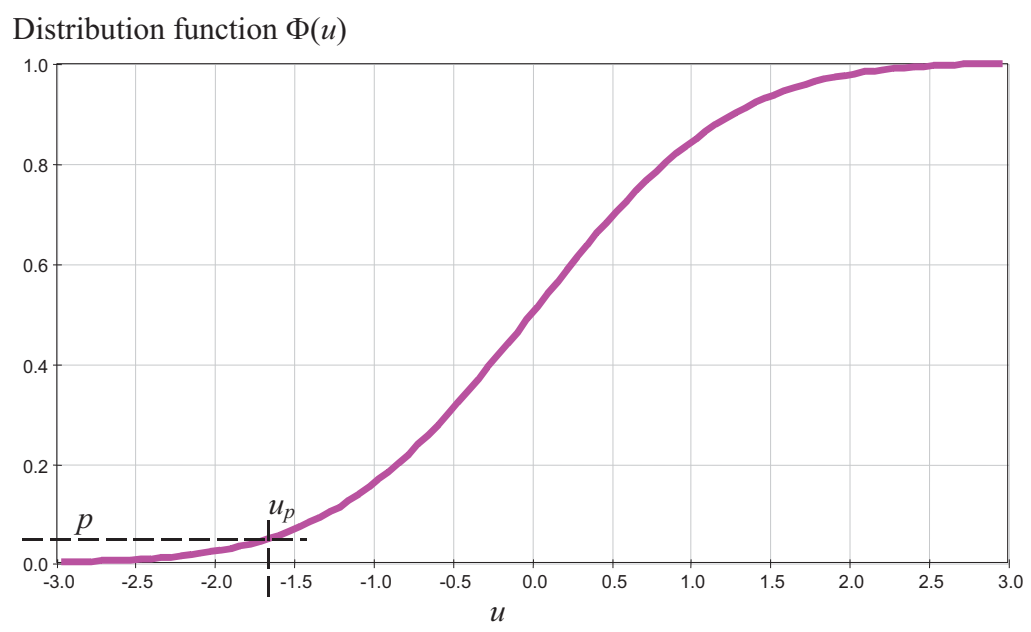

Probability density $\varphi(u)$

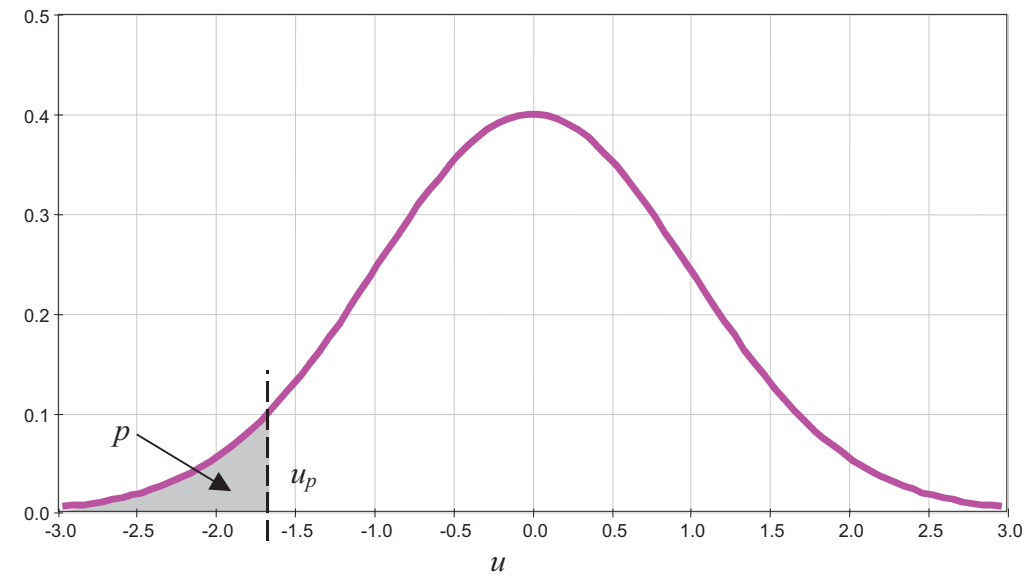

Figure 4.1. Definition of the fractile for a standardised random variable $U$. 
Fractiles $u_{p}$ of standardised random variables $U$ are commonly available in tables. Figure 4.1 illustrates the definition of the fractile described by equation (4.1) for a standardised random variable $U$; it shows the distribution function $\Phi(u)$, the probability density function $\varphi(u)$, the probability $p$ (equal to 0,05 ) and the fractile $u_{p}$ (equal to $-1,645$ ) for the normal standardised distribution of $U$.

In general, the fractile $x_{p}$ of an original random variable $X$ may be calculated using tables for $u_{p}$ available for standardised random variables $U$ with a relevant type of distribution. It follows from the transformation (3.14) that the fractile $x_{p}$ may be determined from the standardised random variable $u_{p}$ (found in available tables) using the relationship

$$
x_{\mathrm{p}}=\mu+u_{p} \sigma=\mu\left(1+u_{p} w\right)
$$

where $\mu$ denotes the mean, $\sigma$ the standard deviation and $w$ the coefficient of variability of the observed variable $X$.

If the probability $p<0,5$, then the value $x_{p}$ is called the lower fractile, for $p>0,5$ the $x_{p}$ is called the upper fractile. Figure 4.2 shows the lower and upper fractiles $u_{p}$ of a standardised random variable $U$ with a normal distribution for probabilities $p=0,05$ and 0,95 , and thus denoted $u_{0,05}$ and $u_{0,95}$.

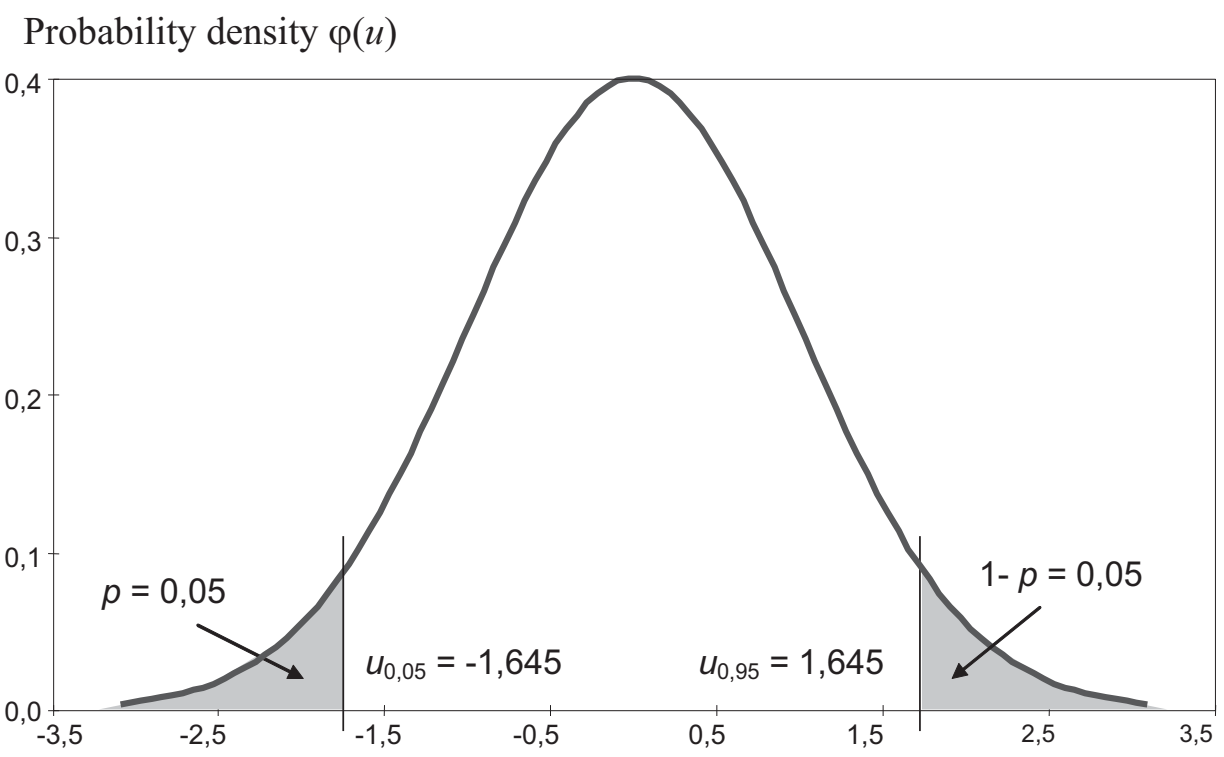

Standardized random variable $U$ having normal distribution

Figure 4.2. The lower and upper fractiles of a standardised random variable $U$ having a normal distribution.

The values $u_{\mathrm{p}}$ of the lower fractile of a standardised random variable $U$ having a normal distribution for selected probabilities $p$ are given in Table 4.1. Considering the symmetry of the normal distribution, the values $u_{\mathrm{p}}$ of the upper fractile can be assessed from Table 4.1 by the substitution of $p$ with $1-p$ and by changing the sign of values $u_{\mathrm{p}}$ (from negative to positive). Detailed tables can be found, for example in textbooks $[5,6]$, in the standard ISO 12491 [3], and in specialised literature [18, 19, 20]. 
Table 4.1. Fractile $u_{\mathrm{p}}$ of a standardised random variable with a normal distribution.

\begin{tabular}{|l|llllllllll|}
\hline$p$ & $10^{-7}$ & $10^{-6}$ & $10^{-5}$ & $10^{-4}$ & 0,001 & 0,010 & 0,050 & 0,100 & 0,200 & 0,500 \\
$-u_{p}$ & 5,199 & 4,753 & 4,265 & 3,719 & 3,091 & 2,327 & 1,645 & 1,282 & 0,841 & 0,000 \\
\hline
\end{tabular}

For a standardised random variable with a general three-parameter log-normal distribution the value $u_{\mathrm{p}}$ of the standardised random variable is dependent on the skewness $\alpha$. The values $u_{\mathrm{p}}$ for selected skewnesses $\alpha$ and probabilities $p$ are given in Table 4.2.

The fractile corresponding to the probability $p=0,05$ is usually applied for an assessment of the characteristic value of material properties (strength of concrete, yield strength of steel, masonry strength). However, the design values of dominant variables are fractiles which correspond to a lower probability ( $p \cong 0,001)$, the design values of nondominant variables are fractiles corresponding to a greater probability $(p \cong 0,10)$.

Table 4.2. Fractile $u_{\mathrm{p}}$ of a standardised random variable with a log-normal distribution.

\begin{tabular}{|l|ccccccccccccc|}
\hline & \multicolumn{10}{|c|}{ Probability $p$} \\
\hline & $10^{-4}$ & $10^{-3}$ & 0,01 & 0,05 & 0,10 & 0,20 & 0,50 & 0,80 & 0,90 & 0,95 & 0,99 & $1-10^{-3}$ & $1-10^{-4}$ \\
\hline$-2,0$ & $-9,52$ & $-6,24$ & $-3,52$ & $-1,89$ & $-1,24$ & $-0,61$ & 0,24 & 0,77 & 0,97 & 1,10 & 1,28 & 1,42 & 1,49 \\
$-1,5$ & $-7,97$ & $-5,51$ & $-3,31$ & $-1,89$ & $-1,29$ & $-0,68$ & 0,20 & 0,81 & 1,04 & 1,21 & 1,45 & 1,65 & 1,77 \\
$-1,0$ & $-6,40$ & $-4,70$ & $-3,03$ & $-1,85$ & $-1,32$ & $-0,74$ & 0,15 & 0,84 & 1,13 & 1,34 & 1,68 & 1,99 & 2,19 \\
$-0,5$ & $-4,94$ & $-3,86$ & $-2,70$ & $-1,77$ & $-1,32$ & $-0,80$ & 0,08 & 0,85 & 1,21 & 1,49 & 1,98 & 2,46 & 2,81 \\
0,0 & $-3,72$ & $-3,09$ & $-2,33$ & $-1,65$ & $-1,28$ & $-0,84$ & 0,00 & 0,84 & 1,28 & 1,65 & 2,33 & 3,09 & 3,72 \\
0,5 & $-2,81$ & $-2,46$ & $-1,98$ & $-1,49$ & $-1,21$ & $-0,85$ & $-0,08$ & 0,80 & 1,32 & 1,77 & 2,70 & 3,86 & 4,94 \\
1,0 & $-2,19$ & $-1,99$ & $-1,68$ & $-1,34$ & $-1,13$ & $-0,84$ & $-0,15$ & 0.74 & 1,32 & 1,85 & 3,03 & 4,70 & 6,40 \\
1,5 & $-1,77$ & $-1,65$ & $-1,45$ & $-1,21$ & $-1,04$ & $-0,81$ & $-0,20$ & 0,68 & 1,29 & 1,89 & 3,31 & 5,51 & 7,97 \\
2,0 & $-1,49$ & $-1,42$ & $-1,28$ & $-1,10$ & $-0,97$ & $-0,77$ & $-0,24$ & 0,61 & 1,24 & 1,89 & 3,52 & 6,24 & 9,52 \\
\hline
\end{tabular}

In the case of a log-normal distribution with the lower bound at zero, which is described in section 3.4, it is possible to calculate the fractile from the value of the fractile of a standardised random variable with a normal distribution using the relation

$$
x_{p}=\frac{\mu}{\sqrt{1+w^{2}}} \exp \left(u_{n o r m, p} \sqrt{\ln \left(1+w^{2}\right)}\right)
$$

where $u_{\text {norm,p }}$ is the fractile of a standardised random variable with a normal distribution, $\mu$ is the mean and $w$ the coefficient of variation of the variable $X$. An approximation of equation (4.3) is often applied in the form

$$
x_{p} \cong \mu \exp \left(u_{\text {norm }, p} \times w\right)
$$

whose accuracy is fully satisfying for $w<0,2$, but it is commonly used for greater $w$ as well.

\section{Example 4.1}

Let us assess the fractile $x_{\mathrm{p}}$ of a normal and log-normal distribution with the lower limit at zero for $p=0,001 ; 0,01 ; 0,05$ and 0,10 , if $w=0,3$. We know that the log-normal distribution with the lower limit at zero has, in this case, a positive skewness $\alpha=0,927$ (according to equation (3.23)), which needs to be known for interpolation in Table 4.2. The resultant values $x_{\mathrm{p}}$ are given in the following table in the form of dimensionless coefficients $x_{\mathrm{p}} / \mu$ (expressing the ratio of the fractile to the mean), which were assessed in different ways for the normal and for the log-normal distribution. 
Table of the fractions $x_{p} / \mu$.

\begin{tabular}{|l|rrrr|}
\hline \multicolumn{1}{|c|}{ Fraction $x_{p} / \mu$ for } & \multicolumn{4}{c|}{ Probability $p$} \\
& 0,001 & 0,010 & 0,050 & 0,100 \\
\hline normal distribution, equation (4.2) and Table 4.1 & 0,073 & 0,302 & 0,506 & 0,615 \\
\hline log-normal distribution, equation (4.2) and Table 4.2 & 0,385 & 0,483 & 0,591 & 0,658 \\
\hline log-normal distribution, equation (4.3) and Table 4.1 & 0,387 & 0,484 & 0,591 & 0,657 \\
\hline $\log$-normal distribution, equation (4.4) and Table 4.1 & 0,396 & 0,496 & 0,610 & 0,681 \\
\hline
\end{tabular}

The above table of coefficients $x_{p} / \mu$ shows the expected difference between the fractiles of normal and log-normal distributions. The lower fractile of the normal distribution is significantly lower than the corresponding fractile of the log-normal distribution, particularly for small probabilities $p$. The table also shows that the approximate formula (4.4) provides satisfactory results for computation of the fractile of the log-normal distribution (the error will decrease with decreasing the coefficient of variation $w$ ).

The fractile of the gamma distribution can be calculated from the available tables for the type III Pearson distribution [5,6]. To calculate the fractile of the beta distribution, the available tables of an incomplete beta function may be used or it can be assessed by an integration of the probability density function according to definition (4.1). However, when it is needed (and neither appropriate tables nor software products are available), the fractile of the beta distribution, which is bell shaped (for shape parameters $c>2$ and $d>2$ ), may be assessed approximately from equation (4.2) using the table values of $u_{p}$ for a standardised lognormal distribution, having the same skewness $\alpha$ as the beta distribution. An analogical procedure may be used for other types of distribution too.

The fractile $x_{\mathrm{p}}$ can be easily assessed for the Gumbel distribution. From equation (3.35) and definition (4.1) follows an explicit relation for $x_{p}$ directly dependent on the probability $p$

$$
x_{p}=x_{\bmod }-\frac{1}{c} \ln (-\ln (p)) \cong \mu-(0,45+0,78 \ln (-\ln (p))) \sigma
$$

where the mode $x_{\bmod }$ and parameter $c$ are substituted by relations (3.37) and (3.38).

\section{Example 4.2}

Let us determine the upper fractile of the wind pressure from Example 3.6 described by a Gumbel distribution when a probability $p=0,98$ is considered. It is known from Example 3.6 that for the one-year maximum $\mu_{1}=0,35 \mathrm{kN} / \mathrm{m}^{2}, \sigma_{1}=0,06 \mathrm{kN} / \mathrm{m}^{2}$. The fractile $x_{0,98}$ for such parameters follows from equation (4.5)

$$
x_{0,98}=0,35-(0,45+0,78 \times \ln (-\ln (0,98))) \times 0,06=0,51 \mathrm{kN} / \mathrm{m}^{2}
$$

The corresponding fractile of the maximum for a period of 50 years (as shown in Example 3.6 where $\mu_{50}=0,53 \mathrm{kN} / \mathrm{m}^{2}, \sigma_{50}=0,06 \mathrm{kN} / \mathrm{m}^{2}$ ) is

$$
x_{0,98}=0,53-(0,45+0,78 \times \ln (-\ln (0,98))) \times 0,06=0,69 \mathrm{kN} / \mathrm{m}^{2}
$$

Simple mathematical operations with the Gumbel distribution, including the computation of fractiles, are the main reasons why this distribution is so popular. The Gumbel distribution is frequently used as a theoretical model of random variables describing climatic and other variable actions that are defined on the basis of the maximal values in a given period of time (for example during one or five years). 


\subsection{Fractile estimation from samples - coverage method}

Theoretical models are very rarely known precisely in practical applications. In civil engineering, it is often necessary to assess the fractile of a random variable (for example of the strength of a new or unknown material) from a limited sample, the size $n$ of which may be very small $(n<10)$. Furthermore, random variables may have a high variability (the coefficient of variation is sometimes greater than 0,30 ). The assessment of the fractile of a population from a very small sample is then a serious problem, which is solved in mathematical statistics by various methods of estimation theory. In the following, three basic methods are shortly described: the coverage method, the prediction method and the Bayesian method for estimation of the population fractile.

The keyword of the coverage method for the fractile estimation from a sample of a limited size $n$ is the confidence $\gamma$, i.e. the probability (usually $0,75,0,90$ or 0,95 ) that the estimated value covers the population fractile (that is why the method is called the coverage method). The estimator $x_{p \text {,cover }}$ of the lower fractile $x_{p}$ is determined by the coverage method in such a way that

$$
\mathrm{P}\left(x_{p, \text { cover }}<x_{p}\right)=\gamma
$$

Thus, the estimator $x_{p \text {,cover }}$ is lower (on the safe side) than the unknown fractile $x_{p}$ with the probability (confidence) $\gamma$.

In the following summary practical formulas are given without being derived, assuming that the population has a general three-parameter distribution characterised by the skewness $\alpha$, which is assumed to be known from previous experience. Besides that, it is assumed that the mean $\mu$ of the population is never known in advance and the estimation is based on the average $m$ obtained from a sample. The standard deviation $s$ of the population is assumed to be either known and then it is used, or unknown and then the sample standard deviation $s$ is used instead.

If the standard deviation $\sigma$ of the population is known from previous experience, the estimator $x_{p \text {,cover }}$ of the lower $p$-fractile is given by the relation

$$
x_{p, \mathrm{cover}}=m-\kappa_{p} \sigma
$$

If the standard deviation of the population $\sigma$ is unknown, then the sample standard deviation $s$ is considered

$$
x_{p, \text { cover }}=m-k_{p} s
$$

Coefficients of estimation $\kappa_{p}=\kappa(\alpha, p, \gamma, n)$ and $k_{p}=k(\alpha, p, \gamma, n)$ depend on the skewness $\alpha$, on the probability $p$ corresponding to the fractile $x_{p}$, which is estimated, on the confidence $\gamma$ and on the size $n$ of the population. The knowledge of the confidence $\gamma$ that the estimator $x_{p, \text { cover }}$ will be on the safe side of the real value is the greatest advantage of the classic coverage method. In documents $[1,2]$ the confidence $\gamma$ is recommended by the value 0,75 . In cases of the demands of increased reliability, when a detailed reliability analysis is required, a higher value of confidence, say 0,95 may be more appropriate [14].

\subsection{Fractile estimation from samples - prediction method}

According to the prediction method [14] the lower $p$-fractile $x_{p}$ is estimated by the so-

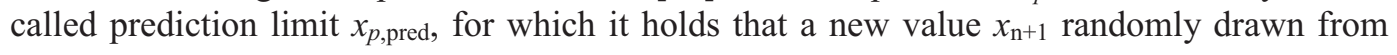


the population will be lower than the estimator $x_{p \text {,pred }}$ only with the probability $p$, i.e. it holds that

$$
\mathrm{P}\left(x_{n+1}<x_{p, \text { pred }}\right)=p
$$

It can be shown that for a growing $n$ the estimator $x_{p \text {,pred }}$ defined in this way is asymptotically approaching the unknown fractile $x_{p}$. It can also be shown that the estimator $x_{p \text {,pred }}$ corresponds approximately to the estimator obtained by the coverage method $x_{p \text {, cover }}$ for a confidence $\gamma=0,75[14]$.

If the standard deviation $\sigma$ of the population is known, then the lower $p$-fractile is estimated by the value $x_{p \text {,pred }}$ according to the relation

$$
x_{p, \text { pred }}=m+u_{p}(1 / n+1)^{1 / 2} \sigma
$$

where $u_{p}=u(\alpha, p)$ is the $p$-fractile of a standardised log-normal distribution having the skewness $\alpha$.

If, however, the standard deviation of the population is unknown, then the sample standard deviation $s$ must be considered instead of $\sigma$

$$
x_{p, \text { pred }}=m+t_{p}(1 / n+1)^{1 / 2} s
$$

where $t_{p}=t(\alpha, p, v)$ is the $p$-fractile of the generalised Student's $t$-distribution for $v=n-1$ degrees of freedom, which has a skewness $\alpha$ (information about the Student's distribution and about the number of degrees of freedom may be obtained from the textbook [5] and from other specialised sources $[18,19,20])$

\subsection{Comparison of the coverage and prediction methods}

The coverage and predictive methods represent two basic procedures of estimation of the population's fractile from an available sample of a limited size $n$. If the standard deviation of the population $\sigma$ is known, then equations (4.7) and (4.10) are applied, in which two analogical coefficients $\kappa_{p}$ and $-u_{p}(1 / n+1)^{1 / 2}$ appear. Both of these coefficients depend on the sample size $n$, the coefficient $\kappa_{p}$ of the coverage method depends more on the confidence $\gamma$. Table 4.3 shows the coefficients $\kappa_{p}$ and $-u_{p}(1 / n+1)^{1 / 2}$ for $p=0,05$ and selected values of $n$ and $\gamma$ when a normal distribution of the population is assumed.

Table 4.3. Coefficients $\kappa_{p}$ and $-u_{p}(1 / n+1)^{1 / 2}$ from equations (4.7) and (4.10) for $p=0,05$ and a normal distribution of the population (when $\sigma$ is known).

\begin{tabular}{c|c|c|c|c|c|c|c|c|c|c}
\hline \multirow{2}{*}{\multicolumn{2}{c|}{ Coefficient }} & \multicolumn{2}{|c|}{ Sample size $n$} \\
\cline { 2 - 10 } \multicolumn{2}{c}{$\kappa_{p}$} & 3 & 4 & 5 & 6 & 8 & 10 & 20 & 30 & $\infty$ \\
\hline \multirow{2}{*}{$-u_{p}(1 / n+1)^{1 / 2}$} & $\gamma=0,75$ & 2,03 & 1,98 & 1,95 & 1,92 & 1,88 & 1,86 & 1,79 & 1,77 & 1,64 \\
& $\gamma=0,90$ & 2,39 & 2,29 & 2,22 & 2,17 & 2,10 & 2,05 & 1,93 & 1,88 & 1,64 \\
& $\gamma=0,95$ & 2,60 & 2,47 & 2,38 & 2,32 & 2,23 & 2,17 & 2,01 & 1,95 & 1,64 \\
\hline
\end{tabular}

It is evident from Table 4.3 that with the growing sample size $n$ both the coefficients approach the value 1,64, which holds for a theoretical model of the normal distribution (see Table 4.1). The coefficient $\kappa_{p}$ of the coverage method increases with increasing confidence $\gamma$. Note that for a confidence $\gamma=0,75$ it holds that $\kappa_{p} \cong-u_{p}(1 / n+1)^{1 / 2}$. Thus, for $\gamma=0,75$ the 
coverage method leads approximately to the same estimator as the predictive method, $x_{p \text {,cover }} \cong$ $x_{p \text {,pred }}$ (for a greater confidence $\gamma>0,75$ the $x_{p \text {,cover }}<x_{p \text {,pred }}$ ).

If the standard deviation of the population $\sigma$ is unknown, equations (4.8) and (4.11) are applied, in which two analogical coefficients $k_{p}$ and $-t_{p}(1 / n+1)^{1 / 2}$ appear. Both of these coefficients depend again on the sample size $n$, the coefficient $k_{p}$ of the coverage method depends more on the confidence $\gamma$. Table 4.4 and Figure 4.3 show the values of coefficients $k_{p}$ and $-t_{p}(1 / n+1)^{1 / 2}$ for $p=0,05$ and selected values of $n$ and $\gamma$ when a normal distribution of the population is assumed.

Table 4.4. Coefficients $k_{p}$ and $-t_{p}(1 / n+1)^{1 / 2}$ from equations (4.8) and (4.11) for $p=0,05$ and a normal distribution of the population (when $\sigma$ is unknown).

\begin{tabular}{l|c|c|c|c|c|c|c|c|c|c}
\hline \multicolumn{2}{l}{ Coefficient } & \multicolumn{10}{c}{ Sample size $n$} \\
\cline { 3 - 11 } \multicolumn{2}{c|}{} & 3 & 4 & 5 & 6 & 8 & 10 & 20 & 30 & $\infty$ \\
\hline \hline \multirow{3}{*}{$k_{p}$} & $\gamma=0,75$ & 3,15 & 2,68 & 2,46 & 2,34 & 2,19 & 2,10 & 1,93 & 1,87 & 1,64 \\
& $\gamma=0,90$ & 5,31 & 3,96 & 3,40 & 3,09 & 2,75 & 2,57 & 2,21 & 2,08 & 1,64 \\
& $\gamma=0,95$ & 7,66 & 5,14 & 4,20 & 3,71 & 3,19 & 2,91 & 2,40 & 2,22 & 1,64 \\
\hline \multicolumn{2}{l|}{$-t_{p}(1 / n+1)^{1 / 2}$} & 3,37 & 2,63 & 2,33 & 2,18 & 2,00 & 1,92 & 1,76 & 1,73 & 1,64 \\
\hline
\end{tabular}

It is obvious from Table 4.4 and Figure 4.3 that with increasing the sample size $n$ both the coefficients $k_{p}$ and $-t_{p}(1 / n+1)^{1 / 2}$ approach the value 1,64, which is valid for a theoretical model of the normal distribution (see Table 4.1). In the case of the coverage method, the coefficient $k_{p}$ increases with increasing confidence $\gamma$ and the relevant estimators $x_{p \text {,cover }}$ of the lower fractile are decreasing (on the safe side). Note that as in the case of the known standard deviation $\sigma$ both coefficients are approximately equal, $k_{p} \cong-t_{p}(1 / n+1)^{1 / 2}$ and for the confidence $\gamma=0,75$ the coverage method leads to approximately the same estimator, $x_{p \text {,cover }} \cong$ $x_{p, \text { pred, }}$ as the prediction method.

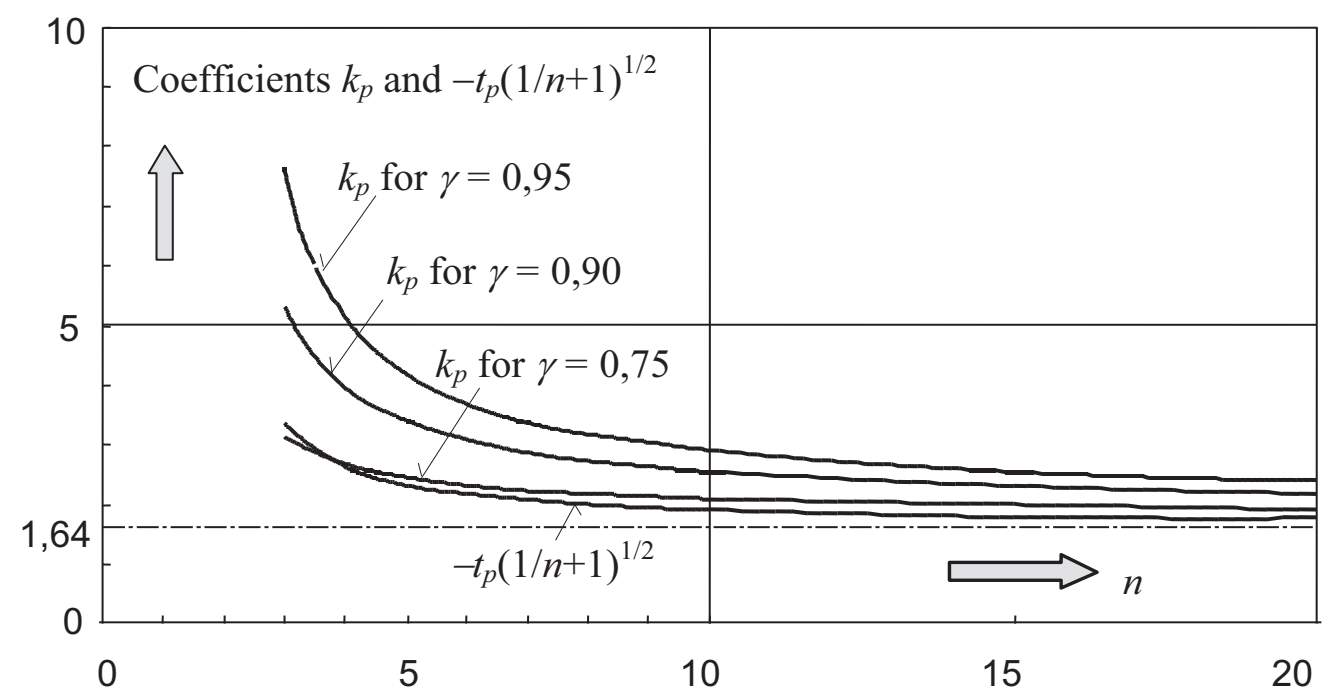

Figure 4.3. Coefficients $k_{p}$ and $-t_{p}(1 / n+1)^{1 / 2}$ for $p=0,05$ and a normal distribution of the population (when $\sigma$ is unknown). 
Also the skewness (asymmetry) of the population $\alpha$ may significantly affect the estimator of the population's fractile. Tables 4.5 and 4.6 show the coefficients $k_{p}$ from equation (4.8) for three values of the skewness $\alpha=-1,0,0,0$ and 1,0, a probability $p=0,05$ and confidences $\gamma=0,75$ (Table 4.5) and $\gamma=0,95$ (Table 4.6). Values of the coefficients from Table 4.6 are shown in Figure 4.4.

Table 4.5. Coefficient $k_{p}$ from equation (4.8) for $p=0,05, \gamma=0,75$ and a log-normal distribution having the skewness $\alpha$ (when $\sigma$ is not known).

\begin{tabular}{l|c|c|c|c|c|c|c|c|c}
\hline \multirow{2}{*}{ Skewness } & \multicolumn{10}{|c}{ Sample size $n$} \\
\cline { 2 - 10 } & 3 & 4 & 5 & 6 & 8 & 10 & 20 & 30 & $\infty$ \\
\hline \hline$\alpha=-1,00$ & 4,31 & 3,58 & 3,22 & 3,00 & 2,76 & 2,63 & 2,33 & 2,23 & 1,85 \\
$\alpha=0,00$ & 3,15 & 2,68 & 2,46 & 2,34 & 2,19 & 2,10 & 1,93 & 1,87 & 1,64 \\
$\alpha=1,00$ & 2,46 & 2,12 & 1,95 & 1,86 & 1,75 & 1,68 & 1,56 & 1,51 & 1,34 \\
\hline
\end{tabular}

Table 4.6. Coefficient $k_{p}$ from equation (4.8) for $p=0,05, \gamma=0,95$ and a log-normal distribution having the skewness $\alpha$ (when $\sigma$ is not known).

\begin{tabular}{l|c|c|c|c|c|c|c|c|c}
\hline \multirow{2}{*}{ Skewness } & \multicolumn{10}{|c}{ Sample size $n$} \\
\cline { 2 - 10 } & 3 & 4 & 5 & 6 & 8 & 10 & 20 & 30 & $\infty$ \\
\hline \hline$\alpha=-1,00$ & 10,9 & 7,00 & 5,83 & 5,03 & 4,32 & 3,73 & 3,05 & 2,79 & 1,85 \\
$\alpha=0,00$ & 7,66 & 5,14 & 4,20 & 3,71 & 3,19 & 2,91 & 2,40 & 2,22 & 1,64 \\
$\alpha=1,00$ & 5,88 & 3,91 & 3,18 & 2,82 & 2,44 & 2,25 & 1,88 & 1,77 & 1,34 \\
\hline
\end{tabular}

It is evident from Tables 4.5 and 4.6 that as the sample size $n$ increases, the coefficients $k_{p}$ approach the values of $u_{p}$, which are valid for a theoretical model of the log-normal distribution (see Table 4.2). Thus, the influence of the skewness does not disappear when $n \rightarrow$ $\infty$, and it is especially significant for small samples and a greater confidence $\gamma=0,95$ (see Figure 4.4).

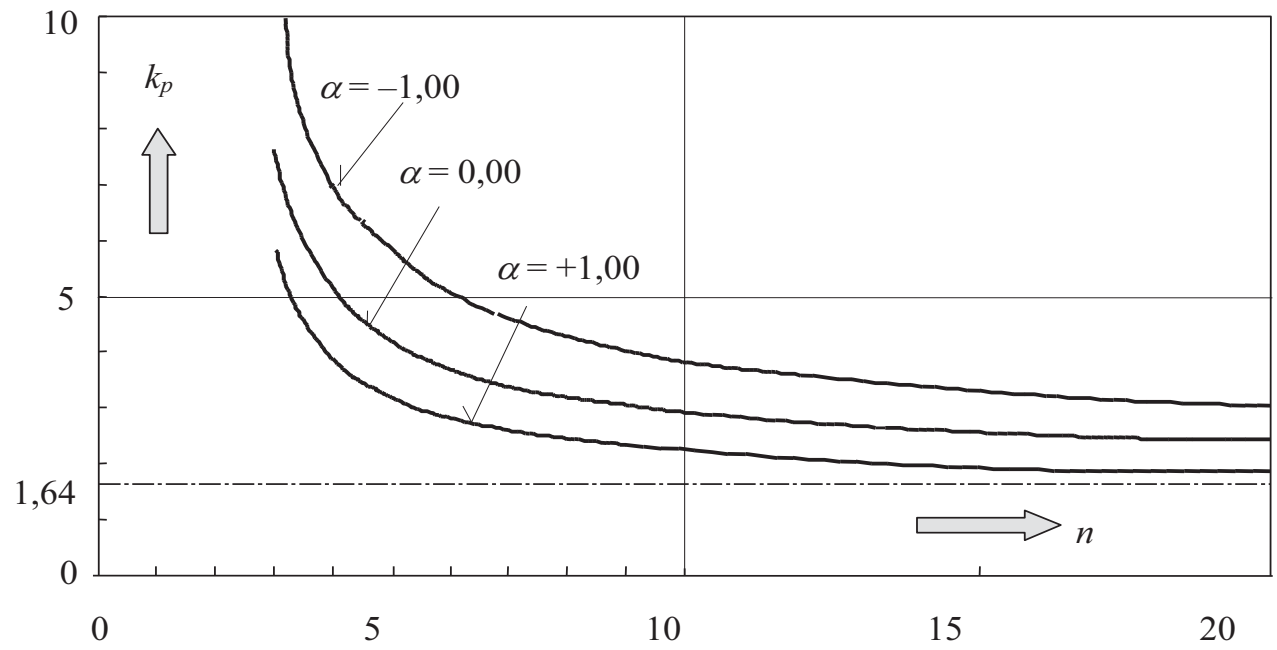

Figure 4.4. Coefficient $k_{p}$ for $p=0,05$ and a confidence $\gamma=0,95$ (when $\sigma$ is unknown). 
A similar dependence on the skewness may be observed in the case of the generalised Student's $t$-distribution for which the fractiles $t_{p}$ are given in Table 4.7. These values $t_{p}$ are applied in the prediction method using formula (4.11) and further in the Bayes method. That is why Table 4.7 gives the values of fractiles $t_{p}$ directly depending on the number of degrees of freedom $v$. As in Tables 4.6 and 4.7 the probability $p=0,05$ and three skewnesses $\alpha=$ $-1,0 ; 0,0$ and 1,0 are considered.

Table 4.7. Coefficient $-t_{p}$ from equation (4.11) for $p=0,05$ and a log-normal distribution with the skewness $\alpha$ (when $\sigma$ is unknown).

\begin{tabular}{l|c|c|c|c|c|c|c|c|c}
\hline \multirow{2}{*}{ Skewness } & \multicolumn{7}{|c}{ Coefficient $-t_{p}$ for $v=n-1$ degrees of freedom } \\
\cline { 2 - 10 } & 3 & 4 & 5 & 6 & 8 & 10 & 20 & 30 & $\infty$ \\
\hline \hline$\alpha=-1,00$ & 2,65 & 2,40 & 2,27 & 2,19 & 2,19 & 2,04 & 1,94 & 1,91 & 1,85 \\
$\alpha=0,00$ & 2,35 & 2,13 & 2,02 & 1,94 & 1,86 & 1,81 & 1,72 & 1,70 & 1,64 \\
$\alpha=1,00$ & 1,92 & 1,74 & 1,64 & 1,59 & 1,52 & 1,48 & 1,41 & 1,38 & 1,34 \\
\hline
\end{tabular}

It follows from Table 4.7 that as the size of the sample $n$ increases, the values of $t_{p}$ approach the theoretical values of $u_{p}$, which are valid for a model of the log-normal distribution with the appropriate skewness and are given in Table 4.2. Therefore, the influence of the skewness again (as in the case of $k_{p}$ ) does not disappear for $n \rightarrow \infty$, but it is especially significant for small samples (it increases with decreasing the sample size $n$ ).

\section{Example 4.3}

A sample of size $n=5$ measurements of the strength of concrete has an average $m=$ 29,2 MPa and a standard deviation $s=4,6 \mathrm{MPa}$. We assume that the population is normal and that its standard deviation $\sigma$ is unknown. The characteristic strength $f_{\mathrm{ck}}=x_{p}$, where $p=0,05$ is firstly assessed by the coverage method. If the confidence is $\gamma=0,75$, then it follows from equation (4.8) and Table 4.4 that

$$
x_{p, \text { cover }}=29,2-2,46 \times 4,6=17,9 \mathrm{MPa}
$$

If a higher confidence $\gamma=0,95$ is required, then

$$
x_{p, \text { cover }}=29,2-4,20 \times 4,6=9,9 \mathrm{MPa}
$$

If the predictive method is used, then it follows from equation (4.11) and Table 4.4 that

$$
x_{p, \text { pred }}=29,2-2,33 \times 4,6=18,5 \mathrm{MPa}
$$

The characteristic strength obtained by the predictive method is only a little greater than the value according to the coverage method with the confidence $\gamma=0,75$. However, if a higher confidence $\gamma=0,95$ is required, then the predictive method leads to a value which is almost twice as great as the value obtained by the coverage method.

If the sample comes from a population with a log-normal distribution and a positive skewness $\alpha=1$, then the coverage method with the confidence $\gamma=0,75$ (Table 4.5) gives an estimator

$$
x_{p, \text { cover }}=29,2-1,95 \times 4,6=20,2 \mathrm{MPa}
$$

which is a value that is $13 \%$ greater than when the skewness is zero.

Similarly it follows for the predictive method from equation (4.11) and Table 4.7 that 


$$
x_{p, \text { pred }}=29,2-1,74 \times \sqrt{\frac{1}{5}+1} \times 4,6=20,4 \mathrm{MPa}
$$

where the value $t_{p}=-1,74$ is given in Table 4.7 for $\alpha=1,0$ and $v=5-1=4$. The resulting strength is in this case $10 \%$ greater than the value which corresponds to the normal distribution $(\alpha=0)$.

\subsection{Fractile estimation from samples - Bayes' method}

If previous experience is available for a random variable (for example in the case of a long-term production), it is possible to use the so-called Bayes method, which generally follows the idea of updating probabilities described in section 2.5. The Bayes method of fractile estimation is described here without deriving any important relations. A more detailed description is given in documents ISO $[2,3]$ and other specialised literature $[18,19,20]$.

Assume that a sample of a size $n$ with an average $m$ and a standard deviation $s$ is available. Besides, an average $m^{\prime}$ ' and a sample standard deviation $s$ ' assessed from an unknown sample of an unknown size $n^{\prime}$ are known from previous experience. It is, however, assumed that both the samples come from the same population having a mean $\mu$ and standard deviation $\sigma$. Then the two samples may thus be combined. This could be a simple task if the individual values of the previous set were known, but that is not the case. However, the Bayes method must be used.

Parameters of the combined sample are generally given by relations $[3,11]$

$$
\begin{gathered}
n^{\prime \prime}=n+n^{\prime} \\
v^{\prime \prime}=v+v^{\prime}-1 \text { if } n^{\prime} \geq 1, v^{\prime \prime}=v+v^{\prime} \text { if } n^{\prime}=0 \\
m^{\prime \prime}=\left(m n+m^{\prime} n^{\prime}\right) / n^{\prime \prime} \\
s^{\prime \prime 2}=\left(v s^{2}+v^{\prime} s^{2}+n m^{2}+n^{\prime} m^{\prime 2}-n^{\prime \prime} m^{\prime \prime}{ }^{2}\right) / v^{\prime \prime}
\end{gathered}
$$

The unknown values $n$ ' and $v^{\prime}$ may be assessed using the relations for the coefficients of variation of the mean and standard deviation $v(\mu)$ and $v(\sigma)$, (parameters $\mu$ and $\sigma$ are considered as random variables in the Bayes concept) for which it holds [2,3]

$$
n^{\prime}=\left[s^{\prime} /\left(m^{\prime} v(\mu)\right)\right]^{2}, v^{\prime}=1 /\left(2 v(\sigma)^{2}\right)
$$

Both the unknown variables $n^{\prime}$ and $v^{\prime}$ are assessed independently (generally $v^{\prime} \neq n^{\prime}-1$ ), depending on previous experience concerning the degree of uncertainty of the estimator of the mean $\mu$ and standard deviation $\sigma$ of the population.

The next step of the procedure applies the prediction method of fractile estimation. The Bayes estimator $x_{p}$,Bayes of the fractile is given by a relationship similar to equation (4.11) for a predictive estimator, assuming that the standard deviation $\sigma$ of the population is not known

$$
x_{p, \text { Bayes }}=m^{\prime \prime}+t_{p}^{\prime \prime}\left(1 / n^{\prime \prime}+1\right)^{1 / 2} s^{\prime \prime}
$$

where $t_{p}^{\prime \prime}=t_{p}^{\prime \prime}\left(\alpha, p, v^{\prime \prime}\right)$ is a fractile of a generalised Student's $t$-distribution having an appropriate skewness $\alpha$, for $v$ " degrees of freedom (that is generally different from the value $n "-1)$.

If the Bayes method is applied for an assessment of material strength, the advantage may be taken of the fact that the long-term variability is constant. Then the uncertainty of an assessment of $\sigma$ and the value $v(\sigma)$ are relatively small, the variables $v$ ' assessed according to 
equation (4.13) and $v$ " assessed according to equation (4.12) are relatively high. This factor may lead to a favourable decrease of the value $t_{p}^{\prime \prime}$ and to an augmentation of the estimator of the lower fractile of $x_{p}$ according to equation (4.14). On the other hand, uncertainties in an assessment of the mean $\mu$ and the variable $v(\mu)$ are usually great and previous information may not significantly affect the resulting values $n$ " and $m$ ".

If no previous information is available, then $n^{\prime}=v^{\prime}=0$ and the resulting characteristics $m$ ", $n$ ", $s$ ", $v^{\prime \prime}$ equal the sample characteristics $m, n, s, v$. In this case the Bayes method is reduced to the prediction method and equation (4.14) becomes equation (4.11); if $\sigma$ is known, equation (4.10) is used. This particular form of the Bayes method, when no previous information is available, is considered in international documents CEN [1] and ISO $[2,3]$.

\section{Example 4.4}

If previous experience were available for example 4.3, the Bayes method could be used. Suppose that the information is

$m^{\prime}=30,1 \mathrm{MPa}, v(\mu)=0,50, s^{\prime}=4,4 \mathrm{MPa}, v(\sigma)=0,28$. It follows from equation (4.13) that

$$
n^{\prime}=\left(\frac{4,4}{30,1} \frac{1}{0,50}\right)^{2}<1, v^{\prime}=\frac{1}{2 \times 0,28^{2}} \approx 6
$$

Further on these values are thus considered: $n^{\prime}=0$ and $v^{\prime}=6$. Because $v=n-1=4$, it follows from equation (4.12)

$$
n "=5, v^{\prime \prime}=10, m ”=29,2 \mathrm{MPa}, s "=4,5 \mathrm{MPa} .
$$

From equation (4.14) the fractile estimate follows as

$$
x_{p, \text { Bayes }}=29,2-1,81 \times \sqrt{\frac{1}{5}+1} \times 4,5=20,3 \mathrm{MPa}
$$

where the value $t_{p}^{\prime \prime}=1,81$ is given in Table 4.7 for $\alpha=0$ and $v^{\prime \prime}=10$. The resulting strength is thus greater (by 10\%) than the value obtained by the predictive method.

If the population has a log-normal distribution with the skewness $\alpha=1$, then it follows from equation (4.14) considering the value $t_{p}^{\prime \prime}=1,48$ given in Table 4.7 that

$$
x_{p, \text { Bayes }}=29,2-1,48 \times \sqrt{\frac{1}{5}+1} \times 4,5=21,9 \mathrm{MPa}
$$

which is a value greater by $8 \%$ than the Bayes estimator for $\alpha=0$.

Examples 4.3 and 4.4 clearly showed that the estimator of characteristic strength (the fractile with a probability $p=0,05$ ) assessed from one sample may be expected within a broad range (in Examples 4.3 and 4.4 from 9,9 $\mathrm{MPa}$ to 21,9 $\mathrm{MPa}$ ), depending on the applied method, required confidence, previous information, and on assumptions concerning the population. Note that besides the alternatives considered in Examples 4.3 and 4.4 concerning confidence level and skewness, knowledge of the standard deviation $\sigma$ of the population and the assumption of a normal distribution or even a negative skewness (in case of some high strength materials) may be applied.

In general, more significant differences in the resulting fractiles may occur when the design values of strength are estimated, i.e. fractiles corresponding to a small probability, than in the case where characteristic values $(p \cong 0,001)$ are considered. However, a direct 
estimation of such fractiles from very small $(n<10)$ or small samples $(10<n<30)$ of the population could be done only if a sufficient amount of information concerning the distribution of the relevant random variable is available. In such a case, it is advisable to compare the results of a direct assessment of the design value with an indirect assessment when the characteristic value is estimated first as a $5 \%$ fractile and then the design value is determined using material partial factors.

\subsection{Estimation of fractiles according to Eurocodes}

Eurocode 1 [1] gives coefficients for estimation of a fractile of a random variable with normal distribution (asymmetric distributions are not considered for the fractile estimation) from a sample for three probabilities $p=0,05$ (for characteristic value $x_{\mathrm{k}}$ ), $p=0,001$ (for the design value $x_{\mathrm{d}}$ of the dominant variable) and for $p=0,10$ (for design value $x_{\mathrm{d}}$ of the nondominating variable). As already mentioned above, the characteristic values $x_{\mathrm{k}}$ and the design values $x_{\mathrm{d}}$ are defined as fractiles $x_{\mathrm{p}}$, which correspond to a given probability $p$ (application of these variables in structural design is explained in the following chapters).

For characteristic values of material properties a fractile corresponding to the probability $p=0,05$ is usually considered (however, for the variables which describe variable loads the probability $p$ is usually less than that), i.e. it holds

$$
\mathrm{P}\left(X<x_{\mathrm{k}}\right)=0,05
$$

For the design values $x_{\mathrm{d}}$ of dominating variables it holds approximately that $p=0,001$ (or another value close to this one), i.e. it holds

$$
\mathrm{P}\left(X<x_{\mathrm{d}}\right)=0,001
$$

Finally, for the design values $x_{\mathrm{d}}$ of non-dominant variables it holds approximately that $p=0,1$, i.e. it holds that

$$
\mathrm{P}\left(X<x_{\mathrm{d}}\right)=0,1
$$

A more detailed description of dominating and non-dominating variables is given in the following chapters.

The following Tables 4.8 and 4.9, which give the required coefficients for the estimation of variables $x_{\mathrm{k}}$ and $x_{\mathrm{d}}$ according to equations (4.15) and (4.15), are adopted from the document [1] in its original version, even though the first Table 4.8 partially overlaps with the precedent Tables 4.3 and 4.4. Table 4.10 is taken from the pre-standard version of EN 1990 (denoted ENV 1991-1). Let us note that all the coefficients in [1] are denoted by the symbol $k_{n}$, which is used also in the following tables.

Table 4.8. Coefficients $k_{n}$ for a 5\% characteristic value (see Tables 4.4 and 4.3).

\begin{tabular}{|c|c|c|c|c|c|c|c|c|c|c|c|}
\hline \multirow[b]{2}{*}{ Coefficient } & \multicolumn{11}{|c|}{ Sample size $n$} \\
\hline & 1 & 2 & 3 & 4 & 5 & 6 & 8 & 10 & 20 & 30 & $\infty$ \\
\hline$-u_{p}(1 / n+1)^{1 / 2}, \sigma$ known & 4,36 & 3,77 & 3,56 & 3,44 & 3,37 & 3,33 & 3,27 & 3,23 & 3,16 & 3,13 & 3,09 \\
\hline$-t_{p}(1 / n+1)^{1 / 2}, \sigma$ unknown & - & - & - & 11,4 & 7,85 & 6,36 & 5,07 & 4,51 & 3,64 & 3,44 & 3,09 \\
\hline
\end{tabular}

\begin{tabular}{l|ccccccccccc}
\hline & \multicolumn{10}{|c}{ Sample size $n$} \\
Coefficient & 1 & 2 & 3 & 4 & 5 & 6 & 8 & 10 & 20 & 30 & $\infty$ \\
\hline$-u_{p}(1 / n+1)^{1 / 2}, \sigma$ known & 2,31 & 2,01 & 1,89 & 1,83 & 1,80 & 1,77 & 1,74 & 1,72 & 1,68 & 1,67 & 1,64 \\
\cline { 1 - 1 }$-t_{p}(1 / n+1)^{1 / 2}, \sigma$ unknown & - & - & 3,37 & 2,63 & 2,33 & 2,18 & 2,00 & 1,92 & 1,76 & 1,73 & 1,64 \\
\hline
\end{tabular}

Table 4.9. Coefficients $k_{n}$ for a design value $x_{\mathrm{d}}$ of a dominating variable, $\mathrm{P}\left(X<x_{\mathrm{d}}\right)=0,001$. 
Table 4.10. Coefficients $k_{n}$ for a design value $x_{\mathrm{d}}$ of a non-dominating variable, $\mathrm{P}\left(X<x_{\mathrm{d}}\right)=0,1$.

\begin{tabular}{|c|c|c|c|c|c|c|c|c|c|c|c|}
\hline \multirow[b]{2}{*}{ Coefficient } & \multicolumn{11}{|c|}{ Sample size $n$} \\
\hline & 1 & 2 & 3 & 4 & 5 & 6 & 8 & 10 & 20 & 30 & $\infty$ \\
\hline$-u_{p}(1 / n+1)^{1 / 2}, \sigma$ known & 1,81 & 1,57 & 1,48 & 1,43 & 1,40 & 1,38 & 1,36 & 1,34 & 1,31 & 1,30 & 1,28 \\
\hline$-t_{p}(1 / n+1)^{1 / 2}, \sigma$ unknown & - & 3,77 & 2,18 & 1,83 & 1,68 & 1,56 & 1,51 & 1,45 & 1,36 & 1,33 & 1,28 \\
\hline
\end{tabular}

The assumption concerning the knowledge of the standard deviation $\sigma$ is replaced (inaccurately) in EN 1990 [1] by the assumption that the coefficient of variation $w$ is known. The original version of Table 4.9 [1] gives for the sample size of $\infty$ a wrong value of 3,08 for the coefficients (the correct value is 3,09 ). Note that when the knowledge of the standard deviation $\sigma$ is assumed, Tables 4.8 to 4.10 give coefficients for the sample size $n=1$. However, application of these values is associated with significant statistical uncertainties and a minimum sample size $n=3$ is recommended here. Note that Table 4.10 (for a 0,1 fractile for a non-dominant random variable) is included only in the previous pre-standard ENV version and not in the final EN 1990 [1].

\subsection{Fractile estimation using updated distribution}

Fractiles may be estimated using updated probability distributions provided that prior distribution $\varphi(x)$ and likelihood function $\varphi(I \mid x)$ are available. Then, using equation (3.70) the updated distribution $\varphi(x \mid I)$ may be found and its appropriate fractile can be determined. The following example of concrete strength shows the practical procedure. Note that the updating procedure may be particularly effective in the case of existing structures.

\section{Example 4.5}

Consider the strength of concrete $\mathrm{C} 20 / 25$ (where $20 \mathrm{MPa}$ is the nominal characteristic strength) for which the log-normal distribution LN $(30,5)$ is commonly assumed as a prior distribution $\varphi(x)$. Let the additional test lead to a likelihood function $\varphi(I \mid x)$ described by lognormal distribution $\operatorname{LN}(31,5 ; 4,6)$ (see Figure 4.5). The updated distribution $\varphi(x \mid I)$, given by equation (3.70), may be approximated by log-normal distribution $\operatorname{LN}(30,3 ; 3,3)$. Compared with the a priori distribution (see Figure 4.5) the updated distribution has a lower variability (the coefficient of variability decreases from 0,167 to 0,109 ) and a greater characteristic strength (5\% fractile), which increases from 22,5 MPa to 25,2 MPa. Obviously updating leads to a considerably greater characteristic strength than that predicted by the a priori distribution. 


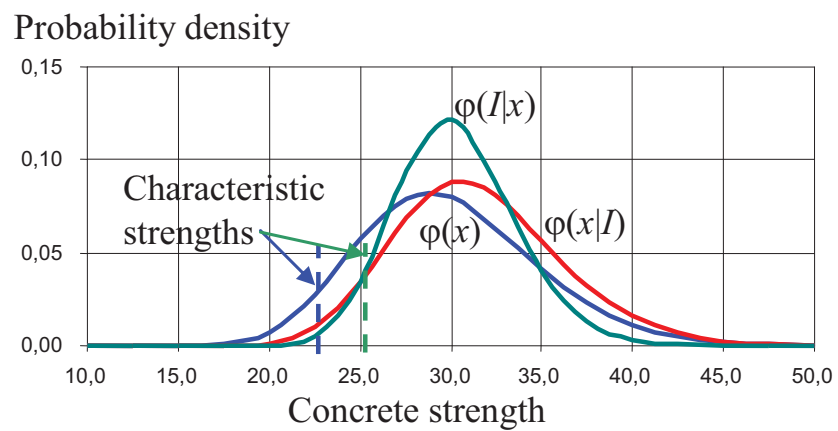

Figure 4.5. Updating of concrete strength distribution. 


\subsection{Basic concepts}

The fundamental task of the theory of structural reliability is the analysis of a simple requirement that the action effect $E$ (expressed in a suitable unit) is smaller than the structural resistance $R$. This condition can be written in the form of the inequality

$$
E<R
$$

The condition (5.1) describes a desirable (satisfactory, safe) state of a considered structural component. It is assumed that structural failure occurs when the condition (5.1) is not satisfied. Thus, an assumed sharp (unambiguous) distinction between a desirable (safe) and undesirable (failure) state of the structure is given by the equality

$$
R-E=0
$$

Equation (5.2) is the fundamental form of the so-called limit state (performance) function. It should be noted, however, that the assumption of a sharp boundary between desirable and undesirable states is a simplification that might not be suitable for all structural members and materials as indicated in the following example 5.1.

\section{Example 5.1}

A steel tie rod from Figure 5.1 has a resistance in axial tension given as $R=\pi d^{2} f_{\mathrm{y}} / 4$, where $d$ denotes the diameter of the $\operatorname{rod}$ and $f_{\mathrm{y}}$ the yield point. The rod is loaded by a weight $E$ $=V \rho$, where $V$ denotes the volume and $\rho$ the density of the suspended mass. Thus the condition (5.1) has the form

$$
V \rho<\pi d^{2} f_{\mathrm{y}} / 4
$$

The limit state function follows from the above inequality as

$$
\pi d^{2} f_{\mathrm{y}} / 4-V \rho=0
$$

In this example, the limit state is defined as the state when the stress in the rod reaches the yield point $f_{\mathrm{y}}$. This simplification is accepted in many common cases, but it may not correspond to the actual failure of the rod, in particular when structural steel with significant ductility and strain hardening is used.

Both the variables $E$ and $R$ are generally random variables and the validity of inequality (5.1) cannot be guaranteed

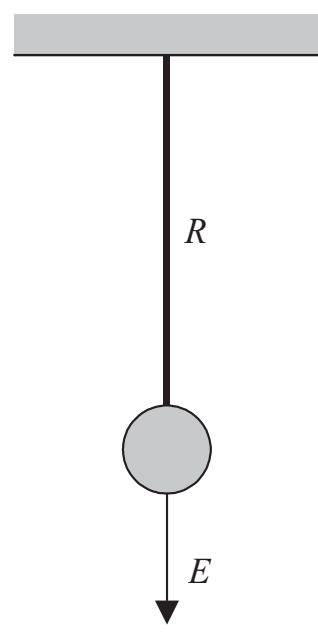

Figure 5.1. A tie rod. absolutely, i.e. with the probability equal to 1 . Therefore, it is necessary to accept the fact that the limit state described by equation (5.2) may be exceeded and failure may occur with a certain probability. The essential objective of reliability theory is to assess the probability of failure $p_{\mathrm{f}}$ and to find the necessary conditions for its limited magnitude. For the simple condition in the form of inequality (5.1), the probability of failure may be formally written as

$$
p_{\mathrm{f}}=\mathrm{P}(E>R)
$$

The random character of the action effect $E$ and the resistance $R$, both expressed in terms of a suitable variable (performance indicator) $X$ (i.e. stress, force, bending moment, deflection) is usually described by an appropriate distribution function, i.e. by distribution 
functions $\Phi_{E}(x), \Phi_{R}(x)$ and by corresponding probability density functions $\varphi_{E}(x), \varphi_{R}(x)$, where $x$ denotes a general point of the considered variable $X$ used to express both of the variables $E$ and $R$. Distributions of the variables $E$ and $R$ further depend on appropriate parameters, for example on moment parameters $\mu_{E}, \sigma_{E}, \alpha_{E}, \mu_{R}, \sigma_{R}$ and $\alpha_{R}$. Let us further assume that $E$ and $R$ are mutually independent (which may be achieved by an appropriate transformation).

Figure 5.2 shows an example of probability density functions of both the variables $E$ and $R$ and their mutual location. Types of distribution and their parameters indicated in Figure 5.2 are just indicative information. In particular, the moment parameters (the means and standard deviations) may be considered as relative values related to the resistance mean $\mu_{R}$ (i.e. normalised by $\mu_{R}$ ).

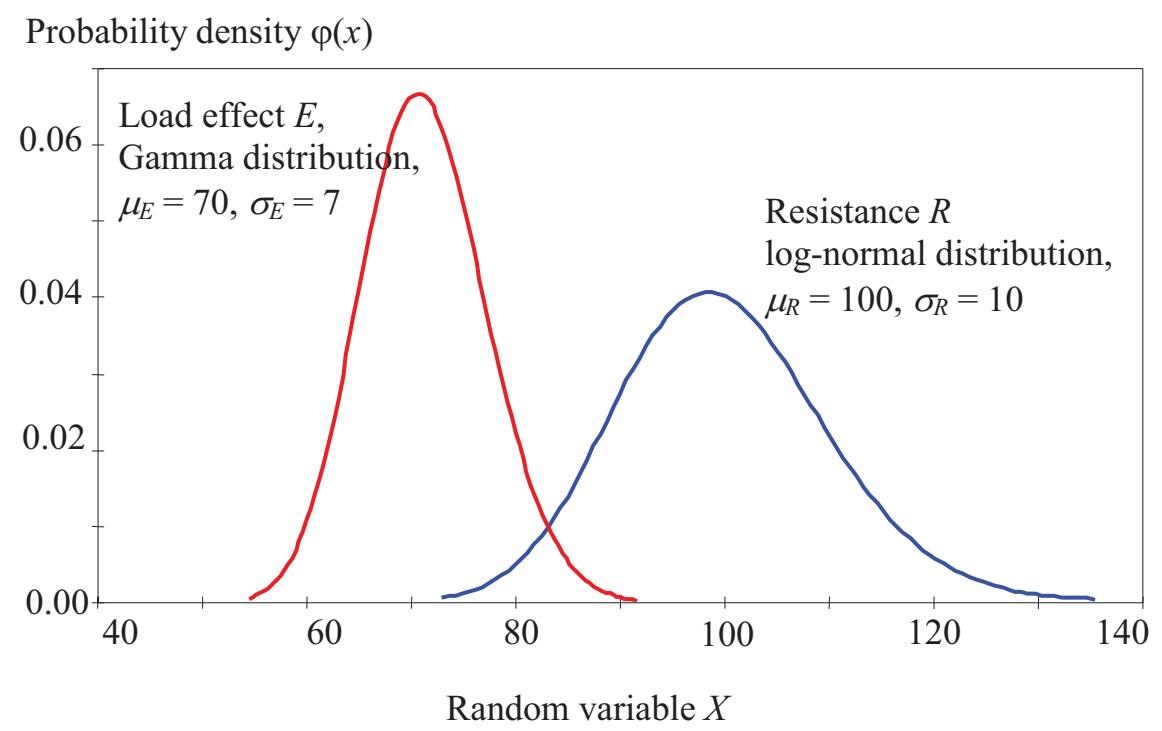

Figure 5.2. Action effect $E$ and resistance $R$ as random variables.

Note that the probability density functions $\varphi_{E}(x)$ and $\varphi_{R}(x)$ shown in Figure 5.2 overlap each other and, therefore, it is clear that unfavourable realisations of the variables $E$ and $R$, denoted by small letters $e$ and $r$, may occur in such a way that $e>r$, i.e. the load effect is greater than the resistance and failure may occur. Obviously in order to keep the failure probability $p_{\mathrm{f}}=\mathrm{P}(E>R)$ within acceptable limits, the parameters of variables $E$ and $R$ must satisfy certain conditions (concerning the mutual location and variances of both the distributions) depending on their types of distribution.

The desired conditions will certainly include the trivial inequality $\mu_{E}<\mu_{R}$ (see Figure 5.2). Obviously, this "requirement for mutual location" of both the distributions is not sufficient to ensure the specified failure probability $p_{\mathrm{f}}$. The correct conditions should obviously also include requirements for variances of both variables. This will be clarified in the following discussion of fundamental cases of structural reliability.

\subsection{Fundamental cases of one random variable}

Consider first a special case when one of the variables $E$ and $R$, say the action effect $E$, has a very low (negligible) variability comparing to the variability of the resistance $R$. Then $E$ may be considered a non-random (deterministic) variable, i.e. a variable that always attains a 
certain fixed value $e_{0}\left(E=e_{0}\right)$. In some cases this assumption may certainly be considered a reasonable approximation. One of these cases may be the loaded tie rod from Example 5.1, where the weight $F$ of the suspended mass can be determined with sufficient accuracy (i.e. without any significant uncertainty). This special case is illustrated in Figure 5.3, where the action effect is indicated by a fixed value $e_{0}=80\left(\mu_{E}=80, \sigma_{E}=0\right)$ and the resistance by the log-normal distribution having the mean $\mu_{R}=80, \sigma_{R}=10$ (all numerical values being normalised dimensionless quantities).

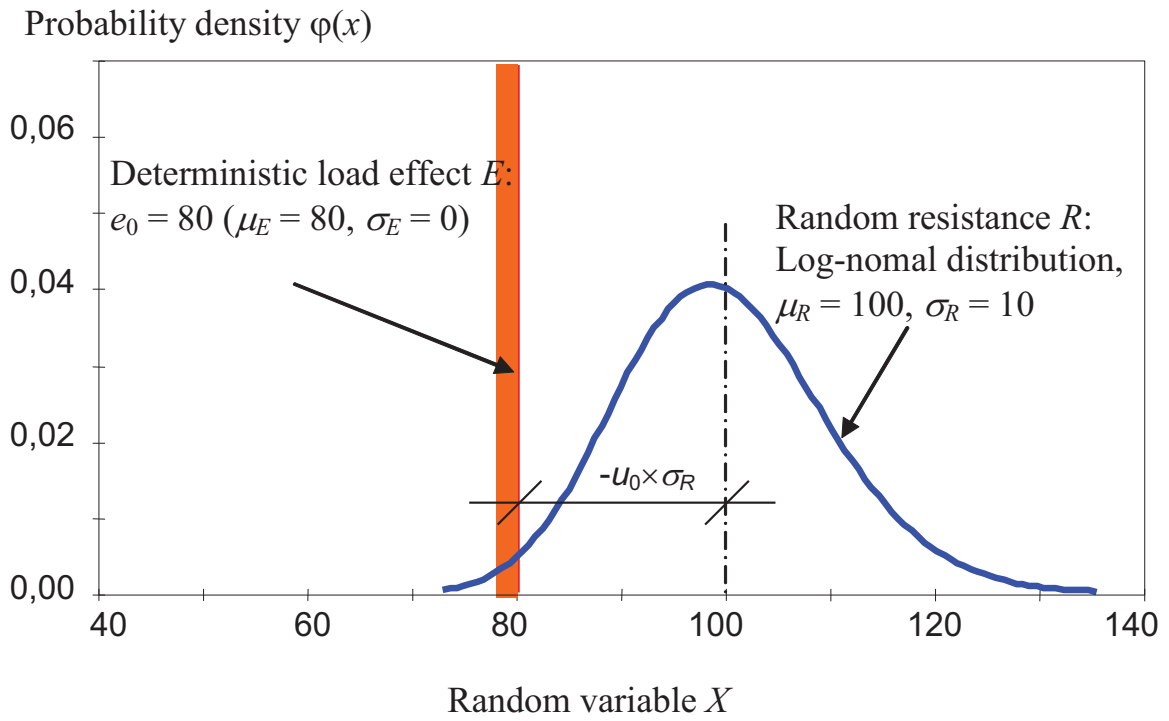

Figure 5.3. The deterministic effect of actions $E$ and random resistance $R$.

The probability of failure $p_{\mathrm{f}}$ for the special case of the deterministic load effect of actions shown in Figure 5.3 may be assessed directly from the distribution function $\Phi_{R}(x)$, similar to the case of a fractile. The value $e_{0}$ may be considered as simply a fractile of the resistance $R$ for which the probability $p_{\mathrm{f}}$ may be calculated using equation (3.1)

$$
p_{\mathrm{f}}=\mathrm{P}\left(R<e_{0}\right)=\Phi_{R}\left(e_{0}\right)
$$

The distribution function $\Phi_{R}\left(e_{0}\right)$ is usually assessed from tables for a standardised random variable $U$, for which the value $u_{0}$ corresponding to $e_{0}$ is computed. It follows from the transformation formula (3.14) that

$$
u_{0}=\left(e_{0}-\mu_{R}\right) / \sigma_{R}
$$

Then the probability of failure is given as

$$
p_{\mathrm{f}}=\mathrm{P}\left(R<e_{0}\right)=\Phi_{\mathrm{LN}, R}\left(e_{0}\right)=\Phi_{\mathrm{LN}, U}\left(u_{0}\right)
$$

where $\Phi_{\mathrm{LN}, U}\left(u_{0}\right)$ is the value of the distribution function of a standardised random variable of the log-normal distribution.

Note that the value $-u_{0}$ is the distance of the fixed value $e_{0}$ of the action effect $E$ from the mean $\mu_{R}$ of the resistance $R$ expressed in the units of the standard deviation $\sigma_{R}$. If the distribution of the resistance $R$ is normal (not log-normal), then the defined distance is called the reliability index $\beta$ 


$$
\beta=\left(\mu_{R}-e_{0}\right) / \sigma_{R}
$$

and the probability of failure may be expressed by the relation

$$
p_{\mathrm{f}}=\mathrm{P}\left(R<e_{0}\right)=\Phi_{U}(-\beta)
$$

If the resistance $R$ has a different distribution from normal, then the reliability index $\beta$ is formally defined as a negative value of a standardised random variable corresponding to the failure probability $p_{\mathrm{f}}$. Thus, in general

$$
\beta=-\Phi_{U}^{-1}\left(p_{f}\right)
$$

where $-\Phi_{U}^{-1}\left(p_{f}\right)$ denotes the inverse distribution function of a standardised normal distribution. As its numerical values are more suitable than the values of the failure probability, the reliability index $\beta$ defined by equation (5.9) is a commonly used measure of structural reliability.

\section{Example 5.2}

Consider that resistance $R$ has a mean $\mu_{R}=100$ (expressed in dimensionless units), standard deviation $\sigma_{R}=10$ (the coefficient of variation is thus $w=0,10$ ). For the deterministic effect of actions it holds that $e_{0}=80$ (see Figure 5.3). If $R$ has a normal distribution, then the reliability index follows directly from equation (5.7)

$$
\beta=(100-80) / 10=2
$$

and the probability of failure follows from relation (5.8)

$$
p_{\mathrm{f}}=\mathrm{P}(R<80)=\Phi_{U}(-2)=0,023
$$

where $\Phi_{u}(-2)$ is the value of the distribution function of the standardised normal distribution.

However, if the distribution of $R$ is not normal but log-normal with the lower bound at zero (according to equation (3.23), the skewness $\alpha=3 w+w^{3}=0,301$ ), then it follows from equation (5.5)

$$
u_{0}=(80-100) / 10=-2
$$

The probability of failure $p_{\mathrm{f}}$ is then given as

$$
p_{\mathrm{f}}=\mathrm{P}(R<80)=\Phi_{\mathrm{LN}, U}(-2)=0,014
$$

where $\Phi_{\mathrm{LN}, U}(-2)$ is the distribution function of the standardised random variable $U$ with the $\log$-normal distribution having the lower bound at zero (the skewness $\alpha=0,301$ ). The resulting probabilities do not differ significantly but this is because their values are rather high.

If the fixed value of an action's effect decreases to $e_{0}=70$, then for the normal distribution of the resistance $R$ the reliability index is $\beta=3$ and the probability of failure is

$$
p_{\mathrm{f}}=\mathrm{P}(R<70)=\Phi_{U}(-3)=0,00135
$$

If the distribution of the resistance $R$ is the log-normal distribution with the lower bound at zero, then

$$
p_{\mathrm{f}}=\mathrm{P}(R<70)=\Phi_{\mathrm{LN}, U}(-3)=0,00021
$$

The reliability index defined by equation (5.9) is $\beta=-\Phi_{U}^{-1}(0,00021)=3,53$, i.e. greater than the value 3 valid for the normal distribution of the resistance $R$.

Obviously, when the load effect is only $e_{0}=70$, the resulting failure probabilities are remarkably lower than in the case when $e_{0}=80$. Furthermore, the numerical example also 
shows that the assumption concerning the type of distribution plays an important role and may be, in some cases, decisive.

\subsection{Two random variables having normal distribution}

Assume that both basic variables, the action effect $E$ and resistance $R$ are random variables. It is then more complicated to assess the probability of failure defined by equation (5.3). A simple solution can be obtained assuming normal distribution for both $E$ and $R$. Then, also, the difference

$$
G=R-E
$$

called the reliability margin, has normal distribution with parameters

$$
\begin{gathered}
\mu_{G}=\mu_{R}-\mu_{E} \\
\sigma_{G}^{2}=\sigma_{R}^{2}+\sigma_{E}^{2}+2 \rho_{R E} \sigma_{R}^{2} \sigma_{E}^{2}
\end{gathered}
$$

Here $\rho_{R E}$ is the coefficient of correlation of $R$ and $E$. It is often assumed that $R$ and $E$ are mutually independent and $\rho_{R E}=0$. Equation (5.3) for the probability of failure $p_{\mathrm{f}}$ can now be modified to

$$
p_{\mathrm{f}}=\mathrm{P}(E>R)=\mathrm{P}(G<0)=\Phi_{G}(0)
$$

and the whole problem is reduced to the determination of the distribution function $\Phi_{G}(0)$, which gives the probabilities of the reliability margin $G$ being negative. The distribution function $\Phi_{G}(0)$ is usually determined using the transformation of the variable $G$ to the standardised random variable $U$ given by equation (3.14). Using this equation, the value $u_{0}$ corresponding to the value $g=0$ is given as

$$
u_{0}=\left(0-\mu_{G}\right) / \sigma_{G}=-\mu_{G} / \sigma_{G}
$$

The probability of failure is then given as

$$
p_{\mathrm{f}}=\mathrm{P}(R<E)=\Phi_{G}(0)=\Phi_{U}\left(u_{0}\right)
$$

The probability density function $\Phi_{G}(g)$ of the reliability margin $G$ is shown in Figure 5.4, where the grey area under the curve $\Phi_{G}(g)$ corresponds to the failure probability $p_{\mathrm{f}}$.

Assuming that $G$ has the normal distribution, the value $-u_{0}$ is called the reliability index, which is commonly denoted by the symbol $\beta$. In the case of the normal distribution of the reliability margin $G$, it follows from equations (5.11), (5.12) and (5.14) that the reliability index $\beta$ is given by a simple relationship

$$
\beta=\mu_{G} / \sigma_{G}=\frac{\mu_{R}-\mu_{E}}{\sqrt{\sigma_{R}^{2}+\sigma_{E}^{2}+2 \rho_{R E} \sigma_{R}^{2} \sigma_{E}^{2}}}
$$

If the quantities $R$ and $E$ are mutually independent, then the coefficient of correlation $\rho_{R E}$ vanishes $\left(\rho_{R E}=0\right)$. Thus, the reliability index $\beta$ is the distance of the mean $\mu_{G}$ of the reliability margin $G$ from the origin, given in the units of the standard deviation $\sigma_{G}$.

\section{Example 5.3}

Consider again the example 5.2, in which the resistance $R$ and the load effect $E$ are mutually independent random variables $\left(\rho_{R E}=0\right)$ having normal distribution. The resistance $R$ has the mean $\mu_{R}=100$, variance $\sigma_{R}=10$ (the coefficient of variation is therefore only $w=$ 
0,10 ), and the effect of actions $E$ has the mean $\mu_{E}=80$ and $\sigma_{E}=8$ (all expressed in dimensionless units). It follows from equation (5.11) and (5.12) that

$$
\begin{gathered}
\mu_{G}=100-80=20 \\
\sigma_{G}^{2}=10^{2}+8^{2}=12,81^{2}
\end{gathered}
$$

As both basic variables $R$ and $E$ have the normal distribution, the reliability index $\beta$ follows directly from equation (5.7)

$$
\beta=20 / 12,81=1,56
$$

and the probability of failure follows from relation (5.8)

$$
p_{\mathrm{f}}=\mathrm{P}(G<0)=\Phi_{U}(-1,56)=0,059
$$

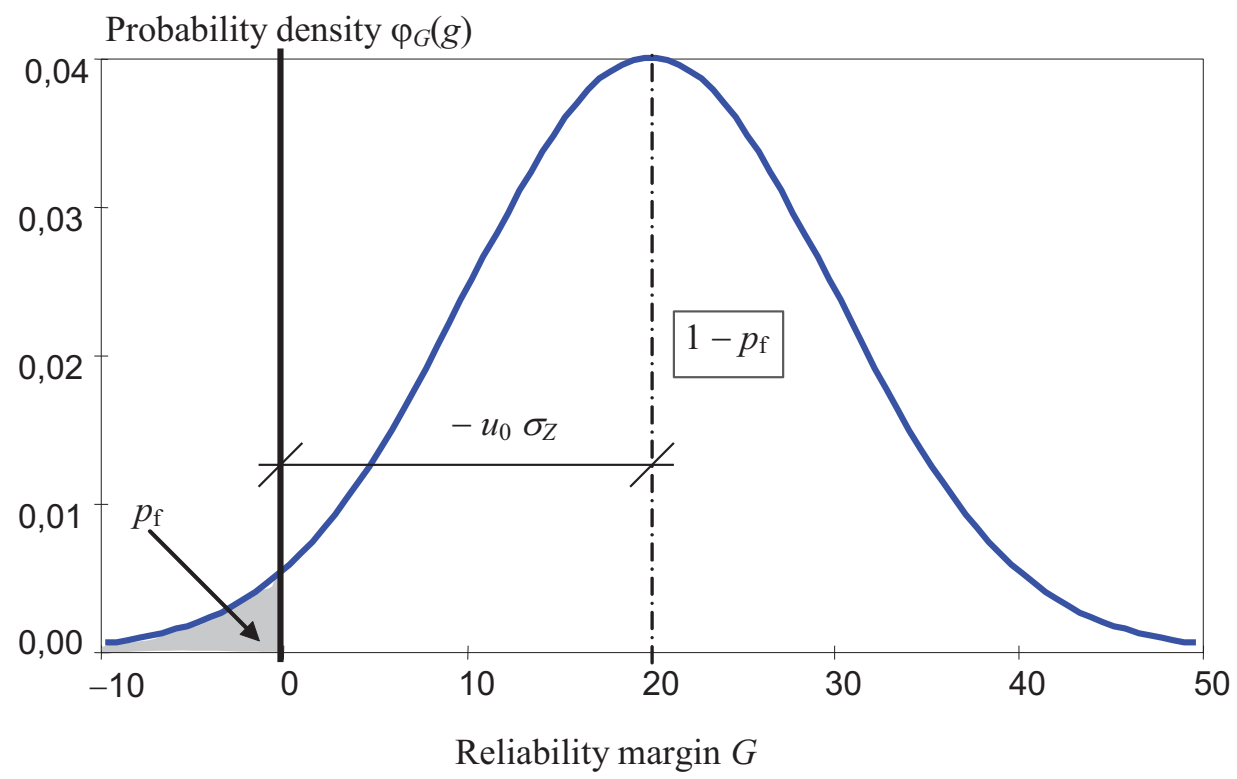

Figure 5.4. Distribution of the reliability margin $G$.

If the variables $E$ and $R$ are not normal, then the distribution of the reliability margin $G$ is not normal either, and the above-described procedure has then to be modified. In a general case, the numerical integration or transformation of both variables into variables with normal distribution can be used. The transformation into normal distribution is used primarily in software products.

There is, however, an approximate simple procedure that can provide a good first assessment of the failure probability $p_{\mathrm{f}}$. The reliability margin $G$ may be approximated by three-parameter log-normal distribution. Assume that the distributions of $E$ and $R$ depend on the moment parameters $\mu_{E}, \sigma_{E}, \alpha_{E}, \mu_{R}, \sigma_{R}$ and $\alpha_{R}$. The mean and the variance of the reliability margin $G$ may be assessed from the previous equations (5.11) and (5.12) which hold for variables with an arbitrary distribution. Assuming mutual independence of $E$ and $R$, the skewness $\alpha_{G}$ of the reliability margin $G$ may be estimated using the following approximate relation 


$$
\alpha_{G}=\frac{\sigma_{R}^{3} \alpha_{R}-\sigma_{E}^{3} \alpha_{E}}{\left(\sigma_{R}^{2}+\sigma_{E}^{2}\right)^{3 / 2}}
$$

It is then assumed that the reliability margin $G$ can be described with sufficient accuracy by a log-normal distribution with determined moment parameters $\mu_{G}, \sigma_{G}$ and $\alpha_{G}$ (equations (5.11), (5.12) and (5.17)). It shows that this approximation offers satisfactory results if the probability of failure is not too small.

\section{Example 5.4}

Consider a tie rod having a rigidity $R$ and bearing a suspended load of a weight $E$. Let $R$ be a log-normal variable with origin at zero having the parameters (expressed again in relative dimensionless units) $\mu_{R}=100$ and $\sigma_{R}=10$ (and therefore $\alpha_{R}=0,301$ ), $E$ has the Gumbel distribution with moment parameters $\mu_{E}=50$ and $\sigma_{E}=10$ (it follows from section 3.7 that $\left.\alpha_{E}=1,14\right)$.

The moment parameters of the reliability margin are assessed according to equations (5.11), (5.12) and (5.17)

$$
\begin{gathered}
\mu_{G}=\mu_{R}-\mu_{E}=100-50=50 \\
\sigma_{G}^{2}=\sigma_{R}^{2}+\sigma_{E}^{2}=10^{2}+10^{2}=14,14^{2} \\
\alpha_{G}=\frac{\sigma_{R}^{3} \alpha_{R}-\sigma_{E}^{3} \alpha_{E}}{\left(\sigma_{R}^{2}+\sigma_{E}^{2}\right)^{3 / 2}}=\frac{10^{3} \times 0,301-10^{3} \times 1,14}{\left(10^{2}+10^{2}\right)^{3 / 2}}=-0,30
\end{gathered}
$$

For a standardised random variable it follows from equation (5.14) that

$$
u_{0}=-\mu_{G} / \sigma_{G}=-50 / 14,14=-3,54
$$

For the log-normal distribution having the skewness $\alpha_{G}=-0,30$ it holds that

$$
p_{\mathrm{f}}=\mathrm{P}(R<E)=\Phi_{\mathrm{LN}, \underline{U}}(-3,54)=0,00101
$$

which corresponds to a reliability index $\beta=3,09$. A more precise result obtained by the application of the software $\operatorname{VaP}[25]$ is $p_{\mathrm{f}}=0,00189$.

However, when skewness is not taken into account in the assessment of probability, it follows from the standardised normal distribution that

$$
p_{\mathrm{f}}=\mathrm{P}(R<E)=\Phi_{U}(-3,54)=0,00020
$$

which differs significantly from the result when the log-normal distribution was considered.

\subsection{Two random variables having general distribution}

The exact solution of the probability of failure $p_{\mathrm{f}}$ defined for the case of two random variables $E$ and $R$ by equation (5.3) may be obtained by integration. Figure 5.5 is used to explain the integration procedure. Let the event $A$ denote the occurrence of an action effect $E$ in the differential interval $\langle x, x+\mathrm{d} x\rangle$. The probability of the event $A$ is given as

$$
\mathrm{P}(A)=\mathrm{P}(x<E<x+\mathrm{d} x)=\varphi_{E}(x) \mathrm{d} x
$$

Let us denote $B$ as the event that a resistance $R$ occurs within the interval $\langle-\infty, x>$. Probability of the event $B$ is, according to section 3.1 , given by the relation

$$
\mathrm{P}(B)=\mathrm{P}(R<x)=\Phi_{R}(x)
$$


The differential increment of the probability of failure $\mathrm{d} p_{\mathrm{f}}$ corresponding to the occurrence of the variable $E$ in the interval $\langle x, x+\mathrm{d} x\rangle$ is given by the probability of simultaneous occurrence of the events $A$ and $B$, i.e. by the probability of their intersection $A \cap$ $B$. According to the principle of multiplication of probabilities (2.22), it holds that

$$
\mathrm{d} p_{\mathrm{f}}=\mathrm{P}(A \cap B)=\mathrm{P}(A) \mathrm{P}(B)=\mathrm{P}(x<E<x+\mathrm{d} x) \mathrm{P}(R<x)=\varphi_{E}(x) \Phi_{R}(x) \mathrm{d} x
$$

The above-mentioned assumption of mutual independence of the variables $E$ and $R$, and thus also of the events $A$ and $B$, is applied here.

Probability density $\varphi(x)$

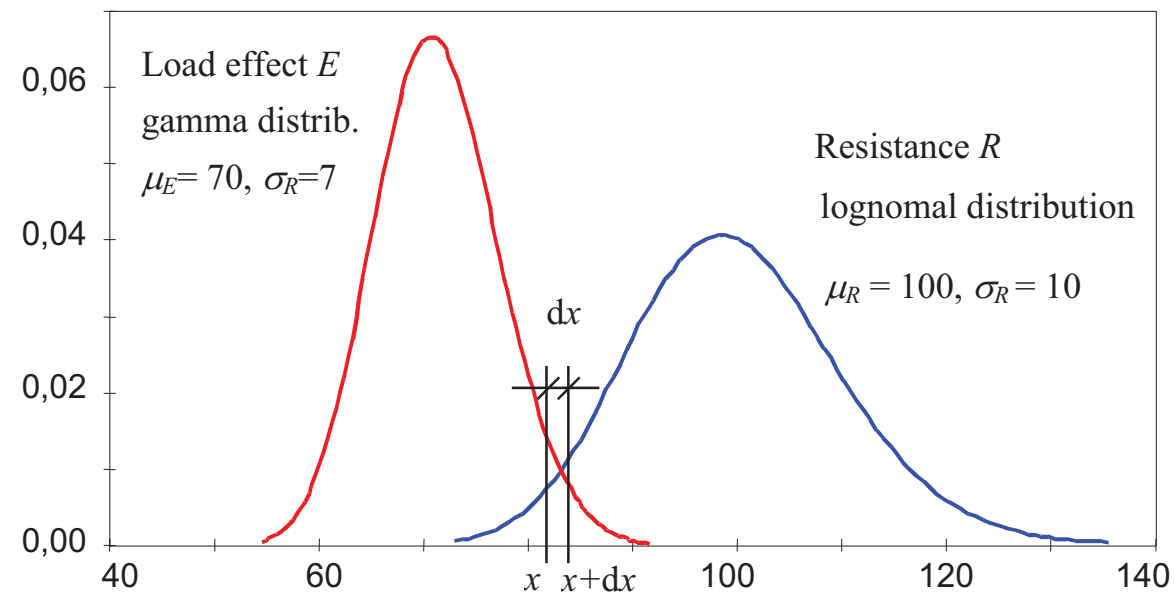

Random variable $X$

Figure 5.5. Distribution of variables $E$ and $R$.

The integration of the differential relationship (5.20) over the interval in which both the variables $E$ and $R$ occur simultaneously (generally the interval $\langle-\infty, \infty\rangle$ ) leads to the relation

$$
p_{\mathrm{f}}=\int_{-\infty}^{\infty} \varphi_{E}(x) \Phi_{R}(x) \mathrm{d} x
$$

The integration of the relation (5.21) usually has to be carried out numerically or using the simulation methods of Monte Carlo.

\section{Example 5.5}

The action effect $E$ and the resistance $R$ are described by a log-normal distribution with the same parameters as in Example 5.4 (the Gumbel distribution for $E$ was simply approximated by a log-normal distribution having the same parameters). The approximate solution in example 5.4, based on the log-normal distribution with the lower bound at zero, leads to the probability of failure $p_{\mathrm{f}}=\mathrm{P}(R<E)=\Phi_{\mathrm{LN}, U}(-3,54)=0,00100$. Numerical integration, according to relation (5.21) using the programme MATHCAD, leads to a solution $p_{\mathrm{f}}=\mathrm{P}(R<E)=0,00187$, the programme VaP suggests a solution $p_{\mathrm{f}}=\mathrm{P}(R<E)=0,00189$, which can be considered a very good approximation. 
Figure 5.6 shows the variation of the probability of failure $p_{\mathrm{f}}$ with the coefficients of skewness $\alpha_{E}$ and $\alpha_{R}$ for the same parameters of variables $E$ and $R\left(\mu_{R}=100, \sigma_{R}=10, \mu_{E}=50\right.$ and $\left.\sigma_{E}=10\right)$ as in Example 5.4 and 5.5. The probability of failure $p_{\mathrm{f}}$ is assessed by direct integration using the programme MATHCAD. It follows from Figure 5.6 that the probability of failure $p_{\mathrm{f}}$ depends greatly on the skewnesses $\alpha_{E}$ and $\alpha_{R}$ (therefore on assumed theoretical models), and in practical conditions, can differ by several orders of magnitude, even when the means and the standard deviations of the variables $E$ and $R$ remain the same.

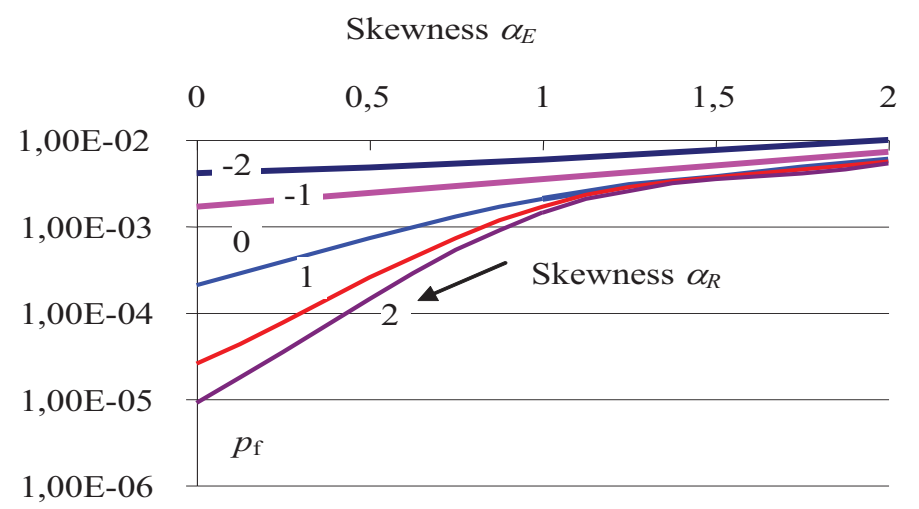

Figure 5.6. Variation of the probability of failure $p_{\mathrm{f}}$ with the coefficients of skewness $\alpha_{E}$ and $\alpha_{R}$ for $\mu_{R}=100, \mu_{R}=10, \sigma_{E}=50$ and $\sigma_{E}=10$.

Thus, it appears that the determination of failure probability in the case of a simple example described by inequality (5.1), where only two random variables $E$ and $R$ are involved, is easy only when both the variables are normally distributed. If they have other distributions, the exact solution is more complicated and the resulting values depend significantly on the assumed types of distributions. The approximate solution assuming for $E$ and $R$ a general (three-parameter) log-normal distribution provides a good first estimate failure probability. The obtained values should be, however, verified by more exact procedures considering appropriate theoretical models of $E$ and $R$.

\subsection{Design point in Eurocodes}

Various simplifications are accepted in order to enable practical application of important principles of the theory of reliability and their effective applications in operational documents like Eurocodes. Figure 5.7, taken from EN 1990 [1], illustrates basic probabilistic principles accepted for developing the partial factor method in Eurocodes. The basic variables $E$ and $R$ considered above are indicated in a two-dimensional graph together with the fundamental limit state function (5.10).

The horizontal axis shows the ratio $R / \sigma_{R}$, the vertical axis the ratio $E / \sigma_{E}$. It is assumed that the variables $E$ and $R$ are independent and both normally distributed. Consequently the joint probability distribution function can be represented by concentric circles corresponding to different levels of the probability density. The assumption of the normal distribution may be in some cases (see examples 5.5 and 5.6) an unrealistic hypothesis, which might provide an approximation only. However, as described later, the actual distributions of both the basic 
variables $E$ and $R$ can be transformed at a given point into the normal distributions and, therefore, Figure 5.7 may be considered as indicating a specific case of variables after such a transformation.

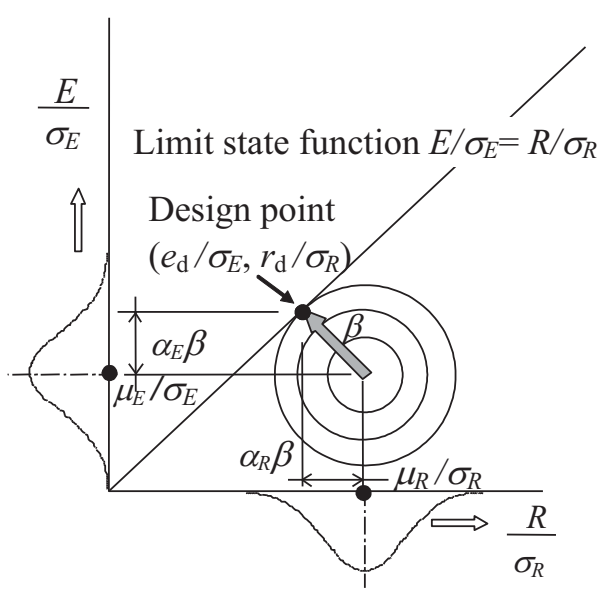

Figure 5.7. Design point.

The safe (desirable) region, where the condition (5.1) is satisfied, is located under the failure boundary (under the diagonal of the axes), the failure (undesirable) region lies above the diagonal. The design point $\left(e_{\mathrm{d}}, r_{\mathrm{d}}\right)$ can be any point lying on the failure boundary (the diagonal). However, it has been shown $[21,22,23,24]$ that the best option is the point on the limit state function closest to the mean $\left(\mu_{E}, \mu_{R}\right)$. Then important properties (like consistency and invariance of the solution under different formulations of the limit state function and choice of basic variables) are assured. Accepting this finding, the design point coordinates follow from Figure 5.7 in the form

$$
\begin{aligned}
& e_{\mathrm{d}}=\mu_{E}-\alpha_{E} \beta \sigma_{E} \\
& r_{\mathrm{d}}=\mu_{R}-\alpha_{R} \beta \sigma_{R}
\end{aligned}
$$

where $\alpha_{E}$ and $\alpha_{R}$ denote here the so-called FORM sensitivity factors of the variables $E$ and $R$, and not the skewness as in the previous sections (such unpleasant ambiguity is accepted in order to use the same notation as the documents CEN and ISO [1,2] ). The "minus" signs in equations (5.22) and (5.23) are used consistently with the convention provided for the sensitivity factors $\alpha_{E}$ and $\alpha_{R}$ in Eurocode [1]).

It follows from Figure 5.7 for the weight factors $\alpha_{E}$ and $\alpha_{R}$ (direction cosines of the normal to the failure boundary), considering the convention in equations (5.22) and (5.23)

$$
\begin{gathered}
\alpha_{E}=-\sigma_{E} / \sqrt{\sigma_{E}^{2}+\sigma_{R}^{2}} \\
\alpha_{R}=\sigma_{R} / \sqrt{\sigma_{E}^{2}+\sigma_{R}^{2}}
\end{gathered}
$$

In Eurocodes an approximation of these sensitivity factors by fixed values is further accepted

$$
\alpha_{E}=-\sigma_{E} / \sqrt{\sigma_{E}^{2}+\sigma_{R}^{2}}=-0,7
$$




$$
\alpha_{R}=\sigma_{R} / \sqrt{\sigma_{E}^{2}+\sigma_{R}^{2}}=0,8
$$

while the validity of such an approximation is delimited in EN 1990 [1] by means of a condition for the ratio of the standard deviations in the form

$$
0,16<\sigma_{E} / \sigma_{R}<7,6
$$

When this condition is not satisfied, it is recommended that the weight factor $\alpha= \pm 1,0$ is used for a variable having the greater standard deviation. Let us remark that this simplification is on the safe side as the sum of squared direction cosines should be equal to 1 .

The design values $e_{\mathrm{d}}$ and $r_{\mathrm{d}}$ of the variables $E$ and $R$ are thus defined as the fractiles of the normal distribution

$$
\begin{aligned}
& \mathrm{P}\left(E>e_{\mathrm{d}}\right)=\Phi_{U}\left(+\alpha_{E} \beta\right)=\Phi_{U}(-0,7 \beta) \\
& \mathrm{P}\left(R<r_{\mathrm{d}}\right)=\Phi_{U}\left(-\alpha_{R} \beta\right)=\Phi_{U}(-0,8 \beta)
\end{aligned}
$$

where $\Phi_{U}(u)$ denotes a standardised distribution function of normal distribution. If $\beta=3,8$, then the design values $e_{\mathrm{d}}$ and $r_{\mathrm{d}}$ are fractiles corresponding approximately to probabilities 0,999 and 0,001 . Note that in equation (5.29) the use of the symmetry of normal distribution is taken into account, i.e. of the relationship $1-\Phi_{U}\left(-\alpha_{E} \beta\right)=\Phi_{u}\left(+\alpha_{E} \beta\right)$.

If the load or resistance models contain several basic variables (other loads, several materials, geometrical data), equations (5.29) and (5.30) are used for the dominating variables (the most significant for the considered structural member) only. For other (non-dominating or accompanying) variables the requirements on the design values are decreased by reducing the reliability index $\beta$ using factor 0.4 , thus

$$
\begin{aligned}
& \mathrm{P}\left(E>e_{\mathrm{d}}\right)=\Phi_{U}\left(+0,4 \alpha_{E} \beta\right)=\Phi_{U}(-0,28 \beta) \\
& \mathrm{P}\left(R<r_{\mathrm{d}}\right)=\Phi_{U}\left(-0,4 \alpha_{R} \beta\right)=\Phi_{U}(-0,32 \beta)
\end{aligned}
$$

If the reliability index $\beta=3,8$, the design values of non-dominant variables are fractiles corresponding approximately to the probabilities 0,9 and 0,1 .

Thus, the design values $e_{\mathrm{d}}$ and $r_{\mathrm{d}}$ are the upper fractiles (for actions) or the lower fractiles (for resistance), corresponding to certain probabilities of being exceeded (actions) or not reached (resistance). For the dominant variables, the probabilities are given by the distribution function of the normal standardised distribution for values $u=+\alpha_{E} \beta$ and $-\alpha_{R} \beta$, in the case of non-dominant variables for reduced values $u=+0,4 \alpha_{E} \beta$ and $-0,4 \alpha_{R} \beta$. These probabilities (for the lower fractile approximately 0,001 for dominant and 0,1 for nondominant variables) are then used to determine the design values of the basic variables having an arbitrary type of (non-normal) distribution. Note that in the case of upper fractiles (actions) the complementary probabilities $(0.999$ and 0.9$)$ and appropriate probability distributions are to be considered (see chapter 4 ).

\section{Example 5.6}

The design values $e_{\mathrm{d}}$ and $r_{\mathrm{d}}$ of the variables $E$ and $R$ from example 5.4 will be assessed assuming that the reliability index $\beta=3,8, \alpha_{E}=-0,7$ and $\alpha_{R}=0,8$. According to equation (5.29), it holds for $E$ that

$$
\mathrm{P}\left(E>e_{\mathrm{d}}\right)=\Phi_{U}\left(\alpha_{E} \beta\right)=\Phi_{U}(-2,66)=0,0039
$$

The complementary probability is therefore 0,9961 and we obtain from equation (4.5)

$$
e_{\mathrm{d}}=\mu-(0,45+0,78 \ln (-\ln (p))) \sigma=50-(0,45+0,78 \times \ln (-\ln (0,9961))) \times 10=88,75
$$


We remark that when the normal distribution is assumed, we obtain from equation (4.5)

$$
e_{\mathrm{d}}=\mu+u_{p} \sigma=50+2,66 \times 10=76,6
$$

According to equation (5.30), it holds for $R$

$$
\mathrm{P}\left(R<r_{\mathrm{d}}\right)=\Phi_{U}\left(-\alpha_{R} \beta\right)=\Phi_{U}(-3,04)=0,0012
$$

For the log-normal distribution with the mean of 100 (units) and the standard deviation of 10 (units) it follows from equation (4.4)

$$
r_{d} \cong \mu \exp \left(u_{\text {norm }, p} \times w\right)=100 \times \exp (-3,04 \times 0,10)=73,79
$$

For the normal distribution we obtain

$$
r_{d}=\mu+u_{p} \sigma=50-3,04 \times 10=69,6
$$

Obviously, it holds for the design point that $e_{\mathrm{d}}>r_{\mathrm{d}}$ and the tie rod does not satisfy the condition (5.1) (we know from example 5.4 that $\beta$ is only 3,09). In order to satisfy the condition for a reliability index of 3,8, the parameters of the variables $E$ and $R$ would have to be modified.

\subsection{Multivariate case}

In previous sections the basic case of two random variables and a linear performance function have been considered. As a rule more basic variables $X_{1}, X_{2}, \ldots X_{n}$ have to be considered. Variables $X_{1}, X_{2}, \ldots X_{n}$ are denoted as the vector $\boldsymbol{X}\left[X_{1}, X_{2}, \ldots X_{n}\right]$ and their realisations $x_{1}, x_{2}, \ldots, x_{n}$ as the vector $\boldsymbol{x}\left[x_{1}, x_{2}, \ldots, x_{n}\right]$. In the multivariate case the reliability margin (5.10) may be generalised as

$$
G\left(X_{1}, X_{2}, \ldots X_{n}\right)=G(\boldsymbol{X})
$$

The safe domain of the basic variables is described by the inequality

$$
G\left(X_{1}, X_{2}, \ldots X_{n}\right)=G(\boldsymbol{X})>0
$$

The unsafe domain of the basic variables is described by the inequality

$$
G\left(X_{1}, X_{2}, \ldots X_{n}\right)=G(\boldsymbol{X})<0
$$

The limit state function is thus given as

$$
G\left(X_{1}, X_{2}, \ldots X_{n}\right)=G(X)=0
$$

When a non-linear performance function $G(\boldsymbol{X})$ and more basic variables $\boldsymbol{X}$ ( $\boldsymbol{X}$ is a vector of basic variables) are considered, failure probabilities $p_{\mathrm{f}}$ can be generally expressed using the limit state function $G(\boldsymbol{X})$ as

$$
P_{\mathrm{f}}=\mathrm{P}(G(\boldsymbol{X}) \leq 0)=\int_{G(\boldsymbol{X}) \leq 0} \varphi(\boldsymbol{X}) \mathrm{d} \boldsymbol{X}
$$

$\varphi(X)$ is the joint probability density function of the vector of all the basic variables $\boldsymbol{X}$ and the inequality $G(X) \leq 0$ denotes the failure domain (the equality $G(X)=0$ denotes the limit state and $G(X) \geq 0$ denotes the safe domain). However, such a function may be difficult to find or may be very complicated. The integral in equation (5.38) can also be written as multiple integral

$$
P_{\mathrm{f}}=\mathrm{P}(G(\boldsymbol{X}) \leq 0)=\int_{G(\mathbf{X}) \leq 0} \varphi_{X 1}\left(x_{1}\right) \varphi_{X 2}\left(x_{2}\right) \ldots \varphi_{X n}\left(x_{n}\right) \mathrm{d} x_{1} \mathrm{~d} x_{2} \ldots \mathrm{d} x_{n}
$$


The integral in equation (5.37) or (5.38) indicates how the probability $p_{\mathrm{f}}$ can be determined provided that the joint probability density function $\varphi(\boldsymbol{X})$ and densities $\varphi_{X i}\left(x_{i}\right)$ are known. In some special cases the integration indicated in equations (5.37) and (5.38) can be done analytically, in some other cases, when the number of basic variables is small (up to 5), various types of numerical integration may be effectively applied.

In general (see ISO 2394 [2]), the failure probability $p_{\mathrm{f}}$ may be computed using:

- exact analytical integration;

- numerical integration methods;

- approximate analytical methods (FORM, SORM, methods of moments);

- simulation methods; or

- by a combination of these methods.

Exact analytical methods can be applied only in exceptional academic cases. Numerical integration can be applied much more frequently. The most popular computational procedures to determine the failure probability constitute approximate analytical methods. In complicated cases simulation methods or their combination with approximate analytical methods are commonly applied. Most of the commercially available software products include approximate analytical methods and various types of simulation methods.

\section{Example 5.7}

To illustrate the general concepts described above consider again the tie rod described in Example 5.1 (see Figure 5.8). The resistance of the rod is given by a non-linear $R=\pi d^{2} f_{\mathrm{y}}$ 14 , where $d$ denotes the diameter of the rod (in general a random variable), $f_{\mathrm{y}}$ is the yield point (a random variable of considerable deviations). Assume that the rod is carrying a deterministic mass $E=F$. The reliability margin (5.33) has therefore the form

$$
G(\boldsymbol{X})=\mathrm{g}\left(d, f_{\mathrm{y}}, F\right)=\pi d^{2} f_{\mathrm{y}} / 4-F>0
$$

The limit state function is given as

$$
G(\boldsymbol{X})=\mathrm{g}\left(d, f_{\mathrm{y}}, F\right)=\pi d^{2} f_{\mathrm{y}} / 4-F=0
$$

In addition to constants there are three basic variables entering the limit state function: $d, f_{\mathrm{y}}$ and $F$. Note that the limit state is defined as reaching the yield point $f_{\mathrm{y}}$, which is a commonly accepted simplification that may not be adequate for some types of construction steel.

The limit state function may be difficult to show graphically in the case of more than two basic variables. For given forces $F=100$ and $50 \mathrm{kN}$ the limit state function is shown as $G(\boldsymbol{X})=0$ in Figure 5.9, where $G(\boldsymbol{X})>0$ corresponds to the safe domain and $G(\boldsymbol{X})<0$ the unsafe domain. The limit state function is a non-linear smooth curve. Figure 5.9 shows also the means of $d$ and $f_{\mathrm{y}}(30 \mathrm{~mm}$ and $290 \mathrm{MPa})$ and the design points, which are derived using the FORM method (described below) and assuming

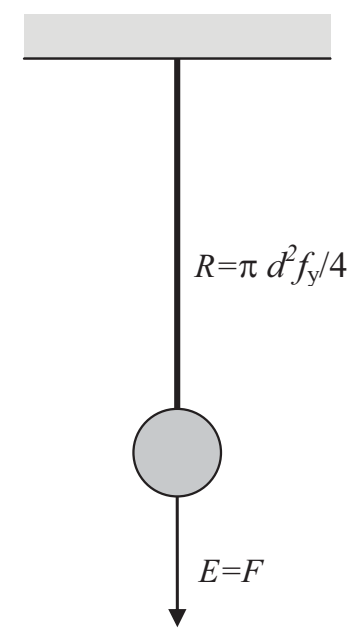

Figure 5.8. A tie rod. normal distribution of $d$ and $f_{\mathrm{y}}$ having standard deviations $3 \mathrm{~mm}$ and $25 \mathrm{MPa}$ respectively.

In accordance with equations (5.37) or (5.38) the failure probabilities $p_{\mathrm{f}}$ can be determined by integration over the unsafe domain, which is located below the curve representing the limit state function (see Figure 5.9). 


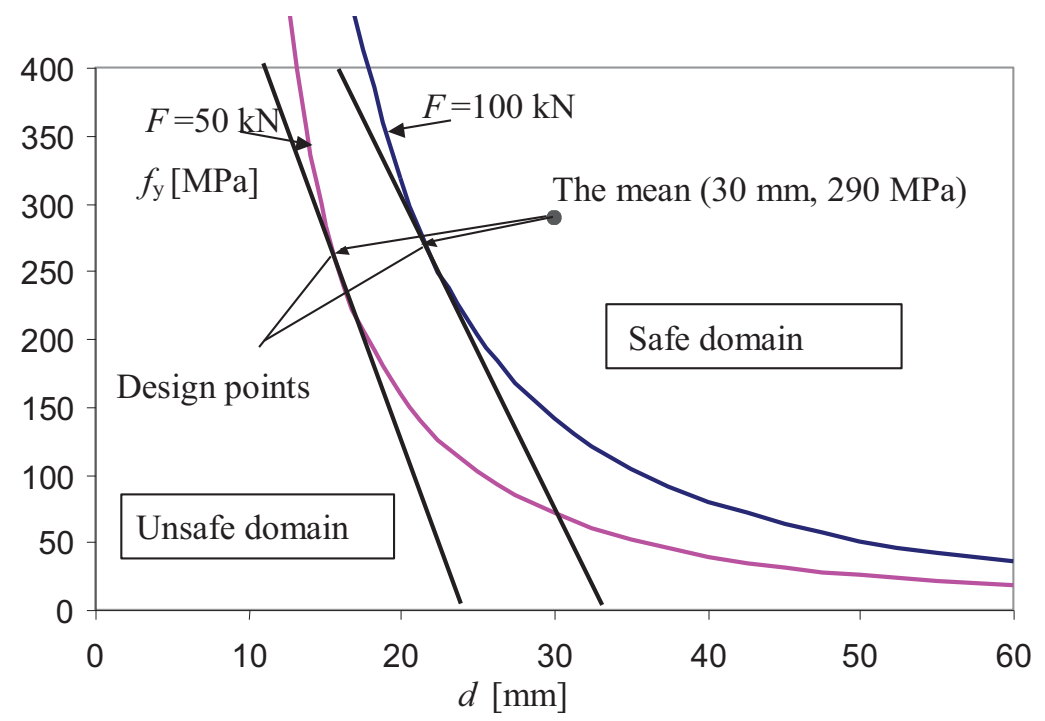

Figure 5.9. The limit state function and design points for the tie rod.

\subsection{FORM and SORM}

The FORM (First Order Reliability Method) is one of the basic and very efficient reliability methods. The FORM method is used as a fundamental procedure by a number of software products for the reliability analysis of structures and systems. It is also mentioned in EN 1990 [1] that the design values are based on the FORM reliability method. Figure 5.7 illustrates this concept for two variables $E$ and $R$. Considering a multivariate case when basic variables are described by a vector $\boldsymbol{X}\left[X_{1}, X_{2}, \ldots X_{n}\right]$, the main steps of the FORM method can be summarised as follows:

- the basic variables $\boldsymbol{X}$ are transformed into a space of standardised normal variables $\boldsymbol{U}$, and the performance function $G(\boldsymbol{X})=0$ transformed into $G^{\prime}(\boldsymbol{U})=0$ (Figure 5.10); - the failure surface $G^{\prime}(U)=0$ is approximated at a chosen given point by a tangent hyperplane (using Taylor expansion);

- the design point, i.e. the point on the surface $G^{\prime}(U)=0$ closest to the origin, is found by iteration (see Figure 5.10);

- the reliability index $\beta$ is determined as the distance of the design point from the origin (see Figure 5.10) and then the failure probability $P_{\mathrm{f}}$ is given as $P_{\mathrm{f}}=\Phi(-\beta)$.

The FORM method can be refined by approximating the failure surface $G^{\prime}(U)=0$ by a quadratic surface. Such a method is called The Second Order Reliability Method (SORM). In literature on structural reliability a number of other improvements and additional modifications may be found [21, 21, 22].

The first step, transformation of the original variable $\boldsymbol{X}$ into a space of standardised normal variables $\boldsymbol{U}$, is illustrated in Figure 5.10 (a) showing the original basic variables $R$ and $E$ and Figure 5.10 (b) showing the transformed variables $U_{R}$ and $U_{E}$. The transformation into the equivalent normal variables at a given point $x *$ is based on two conditions:

- equal distribution functions: 


$$
\Phi_{X}\left(x^{*}\right)=\Phi_{U}\left(\frac{x^{*}-\mu_{X}^{e}}{\sigma_{X}^{e}}\right)
$$

- equal probability increments:

$$
\varphi_{X}\left(x^{*}\right)=\frac{1}{\sigma_{X}^{e}} \varphi_{U}\left(\frac{x^{*}-\mu_{X}^{e}}{\sigma_{X}^{e}}\right)
$$

The mean and the standard deviation of the equivalent normal distribution follow from equations (5.39) and (5.40)

$$
\begin{gathered}
\mu_{X}^{e}=x^{*}-\sigma_{X}^{e}\left[\Phi_{U}^{-1}\left(\Phi_{X}\left(x^{*}\right)\right]\right. \\
\left.\sigma_{X}^{e}=\frac{1}{\varphi_{X}\left(x^{*}\right)} \varphi_{U}\left[\frac{\left.x^{*}-\mu_{X}^{e}\right)}{\sigma_{X}^{e}}\right)\right]=\frac{1}{\varphi_{X}\left(x^{*}\right)} \varphi_{U}\left[\Phi_{U}^{-1}\left(\Phi_{X}\left(x^{*}\right)\right)\right]
\end{gathered}
$$

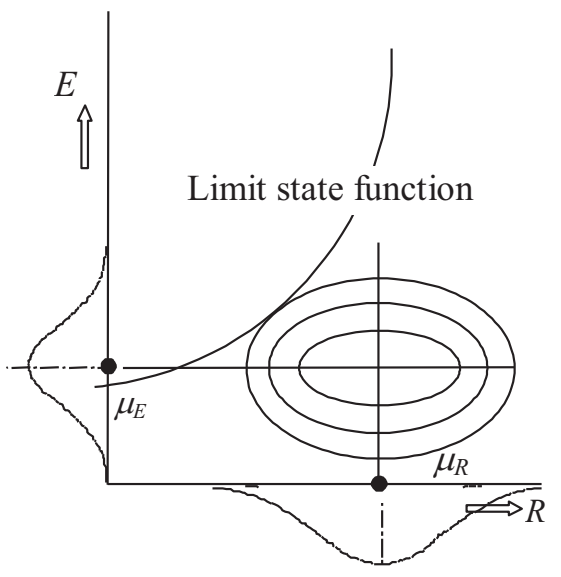

(a) Original basic variables $R$ and $E$.

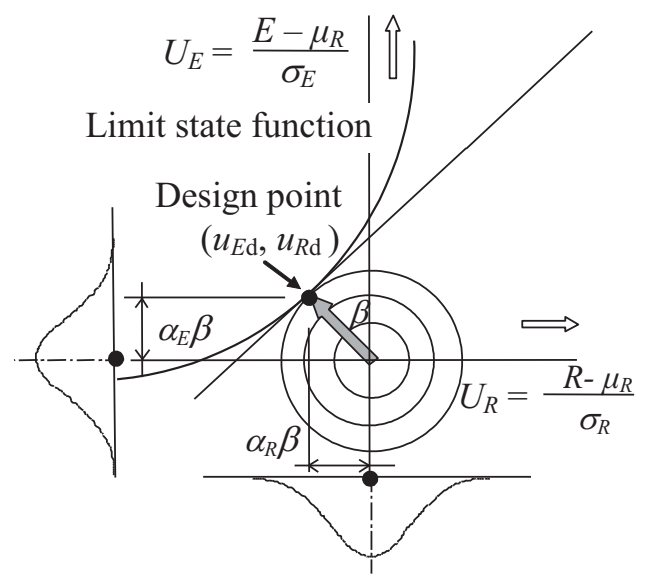

(b) Transformed variables $U_{R}$ and $U_{E}$.

Figure 5.10. First Order Reliability Method.

The whole computation iteration procedure of the FORM method can be summarised in the following ten steps.

1. The limit state function $G(X)=0$ is formulated and theoretical models of basic variables $\boldsymbol{X}=\left\{X_{1}, X_{2}, \ldots X_{n}\right\}$ are specified.

2. The initial assessment of the design point $x^{*}=\left\{x_{1}^{*}, x_{2}{ }^{*}, \ldots x_{n} *\right\}$ is made; for example by the mean values of $\mathrm{n}-1$ basic variables and the last one is determined from the limit state function $G\left(x^{*}\right)=0$.

3. At the point $x^{*}=\left\{x_{1}^{*}, x_{2}^{*}, \ldots x_{n}^{*}\right\}$ equivalent normal distributions are found for all the basic variables using equations (5.37) and (5.38). 
4. The transformed design point $\boldsymbol{u}^{*}=\left\{u_{1}^{*}, u_{2}{ }^{*}, \ldots u_{n}{ }^{*}\right\}$ of the standardised random variables $\boldsymbol{U}=\left\{U_{1}, U_{2}, \ldots U_{n}\right\}$ corresponding to the design point $\boldsymbol{x}^{*}=\left\{x_{1}{ }^{*}, x_{2}{ }^{*}, \ldots x_{n}{ }^{*}\right\}$ is determined using equation

$$
u_{i} *=\frac{x_{i}^{*}-\mu_{X_{i}}^{e}}{\sigma_{X_{i}}^{e}}
$$

5. Partial derivatives denoted as a vector $\boldsymbol{D}$ of the limit state function in respect of the standardised variables $\boldsymbol{U}=\left\{U_{1}, U_{2}, \ldots U_{n}\right\}$ are evaluated at the design point

$$
\boldsymbol{D}=\left[\begin{array}{l}
D_{1} \\
D_{2} \\
\\
D_{n}
\end{array}\right] \text { where } D_{i}=\frac{\partial G}{\partial U_{i}}=\frac{\partial G}{\partial X_{i}} \frac{\partial X_{i}}{U_{i}}=\frac{\partial G}{\partial X_{i}} \sigma_{X_{i}}^{e}
$$

For a linear limit state function $a_{0}+\sum a_{i} X_{i}=0$ the derivatives are $D_{i}=a_{i}$.

6. The reliability index $\beta$ is estimated as

$$
\beta=-\frac{\{D\}^{T}\{u *\}}{\sqrt{\{D\}^{T}\{D\}}} \text { where }\{u *\}=\left\{\begin{array}{l}
u_{1} * \\
u_{2} * \\
\\
u_{n} *
\end{array}\right\}
$$

For a linear limit state function $a_{0}+\sum a_{i} X_{i}=0$ the reliability index is given as

$$
\beta=\frac{a_{0}+\sum a_{i} \mu_{X i}^{e}}{\sqrt{\sum\left(a_{i} \sigma_{X i}^{e}\right)^{2}}}
$$

7. Sensitivity factors are determined as

$$
\{\alpha\}=\frac{\{D\}}{\sqrt{\{D\}^{T}\{D\}}}
$$
from

8. A new design point is determined for $n-1$ standardised and original basic variables

$$
\begin{gathered}
u_{i}^{*}=\alpha_{i} \beta_{i} \\
x_{i}^{*}=\mu_{X_{i}}^{e}-u_{i}^{*} \sigma_{X_{i}}^{e}
\end{gathered}
$$

9. The design value of the remaining basic variable is determined from the limit state function $G\left(x^{*}\right)=0$.

10. The steps 3 to 9 are repeated until the reliability index $\beta$ and the design point $\left\{x^{*}\right\}$ have the required accuracy. 
Note that different sign conventions are used in literature and software products concerning the FORM method. In particular, the sensitivity factors in equations (5.23), (5.24) and the derivatives in (5.47) sometimes have opposite signs to those indicated in the abovementioned equations. The signs of the sensitivity factors and the derivatives of the limit state function used here are consistent with those provided in EN 1990 [1].

\section{Example 5.8}

Consider the tie rod from Example 5.1 where the resistance $R$ has the log-normal distribution $\operatorname{LN}(100,10)$ with the lower bound at zero (the skewness $\alpha_{R}=w_{R}+w_{R}{ }^{3}=0,301$ ) and the load $E$ has the Gumbel distribution $\operatorname{GUM}(50,10)$. Using the iteration procedure indicated above the following results may be obtained: the reliability index $\beta=2,90$, the failure probability $p_{\mathrm{f}}=0,0019$, the design point $e_{\mathrm{d}}=r_{\mathrm{d}}=89,8$, and the sensitivity factors $\alpha_{R}=$ 0,36 and $\alpha_{E}=-0,93$. Almost the same numerical results are obtained when the Gumbel distribution is approximated by the three-parameter log-normal distribution having the skewness $\alpha_{E}=1,14$.

Note that if both the basic variables $R$ and $E$ have the normal distribution, then the reliability index $\beta=3,54$, the failure probability $p_{\mathrm{f}}=0,0002$, the design point $e_{\mathrm{d}}=r_{\mathrm{d}}=75$, and the sensitivity factors $\alpha_{R}=0,707$ and $\alpha_{E}=-0,707$.

\subsection{Simulation methods}

Various simulation methods (direct, adaptive and allocated) are very popular and attractive for their simplicity and transparency. All the simulation methods are based on the generation of random variables of given distribution. Available software products (EXCEL, MATHCAD, MATLAB) include special subroutines for the generation of commonly used types of distributions (uniform, normal, log-normal, Gumbel).

Simulation methods have a number of modifications that can be divided into two basic group:

- zero-one indicator based methods, which operate in the original space of variables $\boldsymbol{X}$,

- conditional expectation methods, which can be called semi-analytical methods.

The first group of the zero-one indicators includes the direct Monte Carlo simulation (when the original probability density is applied), the method of importance sampling (when the original probability density close to the design point is applied) and the adaptive sampling (updated importance sampling). The second group of the conditional expectation consists of directional simulation (suitable in the case of a union of events) and axis orthogonal simulation (suitable in the case of an intersection of events).

In the following the direct Monte Carlo method is described briefly. Information concerning more sophisticated simulation methods are available in a number of specialised references.

Simulation of a random variable $X$ having an arbitrary distribution $\Phi_{X}(x)$ may be in general carried out provided that a generator of random numbers having the uniform distribution in the interval $<0,1\rangle$ is available. If $z_{j}$ denotes realisation of a random $Z$ having the uniform distribution in the interval $<0,1\rangle$, then the corresponding realisation $x_{j}$ of the variable $X$ can be obtained using the inverse of the distribution function $\Phi_{X}^{-1}(z)$, which has the definition domain interval $<0,1>$. Realisations $x_{\mathrm{i}}$ of the random variable $X$ can be therefore obtained from the relationship

$$
x_{j}=\Phi_{X}^{-1}\left(z_{j}\right)
$$


Using equation (5.50), realisations $x_{i j}$ of all basic variables $X_{i}$ can be generated and then it is verified whether a combination of obtained realisations leads to a failure or not. A failure occurs if

$$
G\left(x_{1 i}, x_{2 i}, x_{3 i}, \ldots\right)<0
$$

If the number of all realisations is $n$ and the number of realisations which comply with inequality (5.51) is $n_{\mathrm{f}}$, the failure probability $p_{\mathrm{f}}$ may be assessed using the classical definition of probability based on the ratio

$$
p_{\mathrm{f}}=\frac{n_{\mathrm{f}}}{n}
$$

Obviously, the assessment of the probability $p_{\mathrm{f}}$ is more accurate when the number of realisations $n$ is sufficiently large. A general rule for the specification of the number $n$ is relatively simple. If the expected failure probability is about $10^{-5}$, i.e. from the number of realisations $10^{5}$ on average just one should lead to a failure. Then $n$ should be about two orders greater, thus $n>10^{7}$. Note that the coefficient of variation $w_{p \mathrm{f}}$ of the failure probability can be estimated using formula

$$
w_{p \mathrm{f}}=\left(1-p_{\mathrm{f}}\right)^{0,5}\left(n p_{\mathrm{f}}\right)^{-0,5}
$$

If $p_{\mathrm{f}}=10^{-5}$ and $n=10^{7}$, then it follows from (5.53) that the coefficient of variation is $w_{p \mathrm{f}}=0,10$, which is considered a reasonable accuracy. Clearly, to realise $n=10^{7}$ generations of all the basic variables is a time-consuming, cumbersome procedure. That is why a number of modifications of the direct Monte Carlo have been developed (zero-one indicator-based methods or conditional expectation methods, methods of Latin Hypercube Sampling, or their combination with FORM). These modifications significantly improve the assessment and decrease the number of required realisations. A detailed description of these methods is available in specialised literature and in manuals to the software products COMREL and VaP $[24,25]$.

\section{Example 5.9}

Consider Example 5.4 describing a tie rod $R$ carrying the load $E$. Assume that $R$ has the log-normal distribution with the lower bound at zero, $\mu_{R}=100$ and $\sigma_{R}=10\left(\alpha_{R}=0,301\right)$, the load effect $E$ has the Gumbel distribution having the parameters $\mu_{E}=50$ and $\sigma_{E}=10\left(\alpha_{E}\right.$ $=1,14)$. It is known from Example 5.8 that $E$ may be approximated by the three-parameter log-normal distribution. As the failure probability is expected by the value $p_{\mathrm{f}}=1,910^{-3}$ assessed in Example 5.4 and 5.8, the number of trials should be at least about $10^{5}$. The following Table shows the results obtained by different methods.

\begin{tabular}{|l|c|c|}
\hline Method used for determining $p_{\mathrm{f}}$ & $B$ & $p_{\mathrm{f}}$ \\
\hline Second Moment approximation - Example 5.4 & 3.54 & 0.00020 \\
\hline Third Moment approximation - Example 5.4 & 3.09 & 0.00101 \\
\hline FORM - Example 5.8 & 2.90 & 0.00189 \\
\hline Crude Monte Carlo (100000 trials) & 2.90 & 0.00188 \\
\hline Numerical integration & 2.90 & 0.00187 \\
\hline
\end{tabular}

It follows from the above Table that the second moment approximation provides only a very rough estimate. The third moment approximation seems to provide a much better result. Obviously the most consistent results are obtained by FORM, crude Monte Carlo simulation, and numerical integration. There is practically no difference between the results 
obtained by these methods, and $\beta=2,9$ and failure probability $p_{\mathrm{f}}=0,0019$ can be considered as a sufficiently accurate estimate of the reliability level.

\subsection{Target reliability level}

Probabilistic reliability methods are based on the comparison of the failure probability $p_{\mathrm{f}}$ with its target value $p_{\mathrm{t}}$ or the reliability index $\beta$ with its target value $\beta_{\mathrm{t}}$. It is generally required to design the structure in such a way that the determined values of the basic variables are close to the target values specified in appropriate code provisions, thus

$$
p_{\mathrm{f}} \approx p_{\mathrm{t}}, \text { or } \beta \approx \beta_{\mathrm{t}}
$$

The target values of the reliability index $\beta_{\mathrm{t}}$ given in EN 1990 [1] were derived mainly from a number of previous reliability studies of structural members made from different materials. However, it should be mentioned that the obtained reliability indices depend on many factors (the type of component, loading conditions and structural materials) and, consequently, have a great scatter. It is known that the results of any reliability study are significantly dependent on the assumed theoretical models used to describe the basic variables. Unfortunately these models are not unified and have not been used systematically. Still, the recommended values of the reliability index may be considered as reasonable average values of the reliability level characterising the existing structures.

Another possibility for specifying the target reliability index or the target failure probability is the minimum requirement for human safety from the individual or social point of view when the expected number of fatalities is taken into account. This approach is briefly described in ISO 2394 [2]. Without going into detail it starts from an accepted lethal accident rate of $10^{-6}$ per year, which corresponds to the reliability index $\beta_{\mathrm{t}, 1}=4,7$. This value corresponds to the target reliability index accepted in EN 1990 [1] for an ultimate limit state per one year.

The reliability index for a period of $n$ years may be then calculated from the following approximate equation

$$
\Phi\left(\beta_{\mathrm{t}, n}\right)=\left[\Phi\left(\beta_{\mathrm{t}, 1}\right)\right]^{n}
$$

from which the approximate value $\beta_{\mathrm{t}, 50}=3,8$ may be obtained from $\beta_{\mathrm{t}, 1}=4,7$.

Here $\Phi($.$) denotes the distribution function of a standardised normal distribution.$ Figure 1 shows the variation of $\beta_{n}$ with $\beta_{1}$ for $n=5,25,50$ and 100 . Note that if the reference period $T_{1}$ is one year, then $n$ indicates the number of years of the reference period $T_{n}\left(n=T_{n}\right)$.

Figure 5.11 confirms the data indicated in Table 1.2. For example if the target reliability level of a structure is specified by $\beta_{50}=3,8$ for the design working life $T=50$ years, then it could be verified using the reference period $T_{1}=1$ year and $\beta_{1}=4,7$. When, however, the same reliability index 3,8 is specified for a structure having the design working life $T_{n}=25$ years only, thus $\beta_{25}=3,8$, then the reliability of this structure could be verified using the alternative reference period $T_{1}=1$ year and the reliability index $\beta_{1}=4,5$, similarly when $\beta_{5}=3,8$ then $\beta_{1}=4,2$ (see Figure 5.11).

It should be emphasised that both values $\beta_{\mathrm{t}, 1}=4,7$ and $\beta_{\mathrm{t}, 50}=3,8$ correspond to the same reliability level, but to different reference periods considered for the assessment of the design values of some actions ( 1 and 50 years). The reference period may, or may not, coincide with the design working life.

A completely different question is the specification of the reliability index for a construction works of a limited design working life. A practical illustration of the numerical calculation of the reliability index is shown in Example 5.10. 


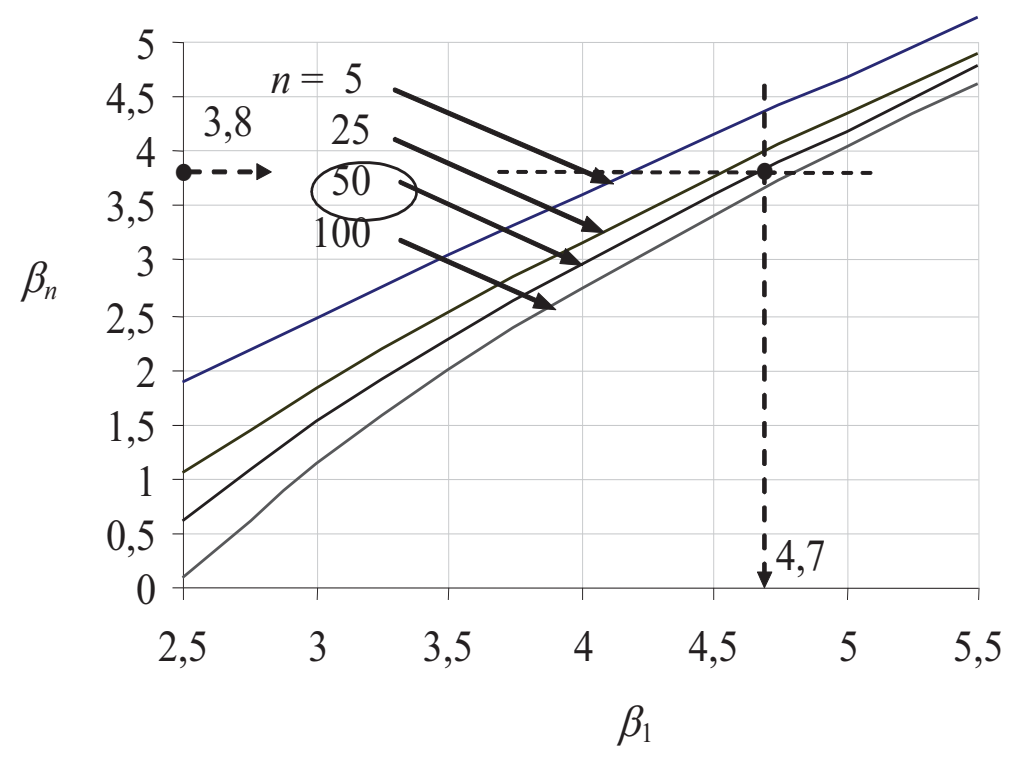

Figure 5.11. Variation of $\beta_{n}$ with $\beta_{1}$ for $n=5,25,50$ and 100 .

It should be noted that the actual frequency of failure may be dependent on many factors not considered in partial factor design and, consequently, the reliability index $\beta$ may not correspond to actual frequency of structural failure.

\section{Example 5.10}

Consider an agricultural structure with moderate consequences of failure and a limited design working life of 25 years. In that case it may be reasonable to specify $\beta_{\mathrm{t}, 1}<4,7$, say $\beta_{\mathrm{t}, 1}$ $=4,2$. Using now the equation (5.55), it can be found that for the design working life $n=25$ years

$$
\Phi(3,4)=[\Phi(4,2)]^{25}
$$

and thus $\beta_{\mathrm{t}, 1}=4,2$ corresponds to $\beta_{\mathrm{t}, 25}=3,4$. Note that using the same equation (5.55) for $n=50$ years, it follows that $\beta_{50}=3,2$. The correct interpretation of this finding is as follows: if the input data (in particular actions) are related to 1 year and the design calculations are done for this period, then $\beta_{1}=4,2$ should be considered; if the input data are related to 25 years, then $\beta_{\mathrm{t}, 1}=3,4$ should be considered in the design verification.

\subsection{Probabilistic optimisation}

Probabilistic optimisation is another way of estimating an adequate reliability level. In general, with increasing reliability level the cost of a structure increases and unfavourable consequences due to structural failure decrease. The basic idea of probabilistic optimisation is to find such a reliability level which would minimize the total cost. To illustrate this concept a simple objective function describing the total cost is considered in the following. Assume that 
the total expected $\cos t C_{\text {tot }}$ of a structural member can be approximated by the objective function

$$
C_{\mathrm{tot}}=C_{0}+C_{P} P+C_{\mathrm{f}} p_{\mathrm{f}}(P)
$$

Here $C_{0}$ denotes the initial cost, which is assumed to be independent of the decisive parameter $P$, the product $C_{P} P$ is the additional cost of the member caused by the chosen magnitude of the parameter $P\left(C_{P}\right.$ denotes the cost per unit parameter $\left.P\right)$, the product $C_{\mathrm{f}} p_{\mathrm{f}}(P)$ is the expected cost caused by the failure of the structural member. The necessary condition for the minimum of the total cost is given by the derivative

$$
\partial C_{\text {tot }} / \partial P=C_{P}+C_{\mathrm{f}} \partial p_{\mathrm{f}}(P) / \partial P=0
$$

Thus the optimum $P$ (if it exists) may be determined from the condition

$$
\partial p_{\mathrm{f}}(P) / \partial P=-C_{P} / C_{\mathrm{f}}
$$

Equation (5.58) can be used in a computer program to determine the optimum value of the parameter $P$. However, it is often less difficult and more transparent to compute the total cost $C_{\text {tot }}$ using equation (5.56) or a similar (more sophisticated) objective function.

\section{Example 5.11}

Consider the reinforced concrete slab described in the appendix to chapter 1. Consider the fundamental objective function (5.56), in which the reinforcement area $A$ is considered the decisive parameter $P$. Figure 5.12 shows the total cost of the slab as a function of the reinforcement area $A$ assuming the partial costs $C_{A}=100, C_{\mathrm{f}}=1000$ of some financial units. The results shown in this example were obtained using the programme COMREL of the software product STRUREL [24].

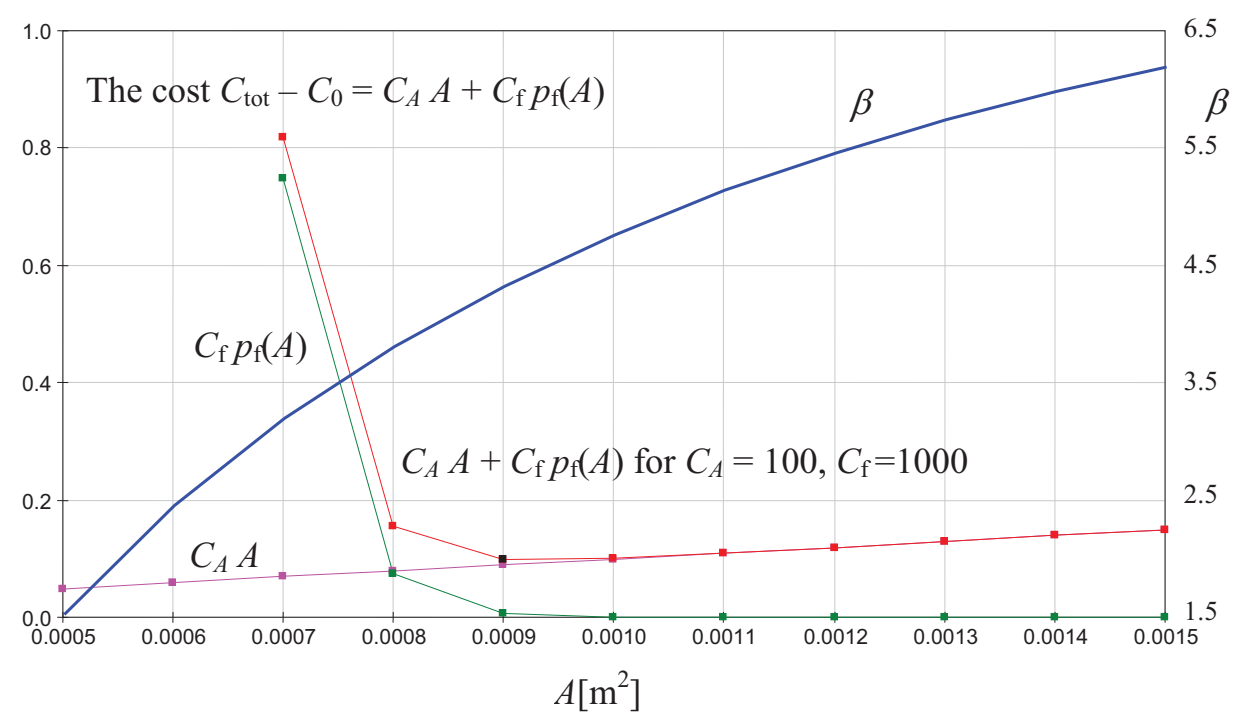

Figure 5.12. Variation of the total cost of the slab and the reliability index $\beta$ with the reinforcement area $A$ assuming the partial costs $C_{A}=100, C_{\mathrm{f}}=1000$ [units]. 
It follows from Figure 5.12 that under the given assumptions the optimum reinforcement area seems to be close to $A=0,0009 \mathrm{~m}^{2}$, which is the value slightly lower than the design area obtained by the partial factor method in the appendix to chapter 1 . Figure 5.12 also shows the variation of the reliability index $\beta$ with the reinforcement area $A$. Note that the optimum reinforcement area $A=0,0009 \mathrm{~m}^{2}$ leads approximately to the reliability index $\beta=$ 4,2 (see Figure 5.12).

Obviously the optimum area $A$ is dependent on the assumed partial costs $C_{A}$ and $C_{\mathrm{f}}$. Figure 5.13 shows the optimum reinforcement areas $A$ for selected partial $\operatorname{costs} C_{A}$ and $C_{\mathrm{f}}$ and the reliability index $\beta$ that is independent of partial costs $C_{A}$ and $C_{\mathrm{f}}$. The cost per unit reinforcement area $C_{A}$ is constant (100 units) and only the cost of failure varies from 10 to 100000 units.

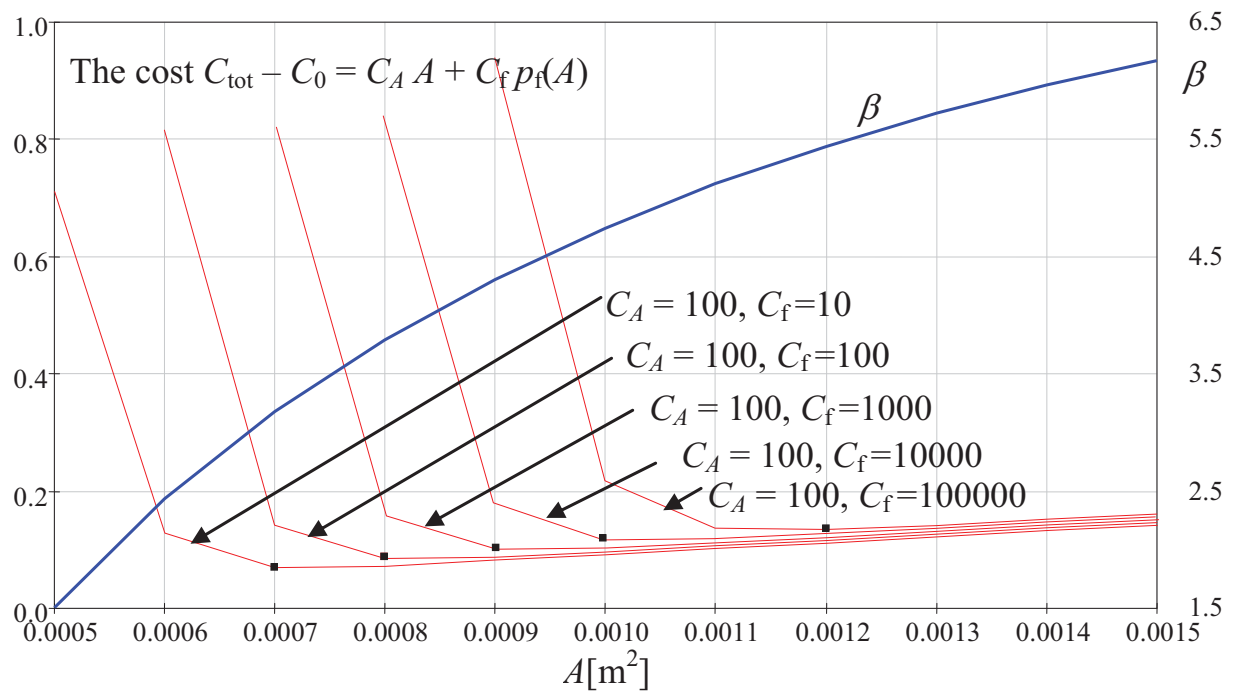

Figure 5.13. Variation of the total cost and the reliability index $\beta$ with the reinforcement area $A$ for selected partial costs $C_{A}$ and $C_{\mathrm{f}}$

It follows from Figure 5.13 that with increasing cost of failure $C_{\mathrm{f}}$ the optimum reinforcement area $A$ and corresponding reliability index increase. It should be noted that for a very low $\operatorname{cost} C_{\mathrm{f}}$ (less than or equal to $C_{A}$ ) the reliability level may be rather low (less than 3,5 ) and may become unacceptable. The reliability requirements on the maximum failure probability may then turn out to be decisive and the optimal reinforcement area $A$ cannot be used. 


\subsection{General considerations}

Time-dependent phenomena affecting the reliability of civil engineering structures and systems are caused primarily by two types of basic variables:

- variable actions (for example imposed and climatic actions),

- material properties (deterioration of construction materials).

In some cases, geometric parameters (including deflections) may also change considerably in time and affect structural reliability. Basic variables, describing time-dependent phenomena (for example wind pressure, snow load, carbonation depth, deflection), are, in general, continuous time-dependent random functions (stochastic processes), which require sophisticated mathematical tools. However, for practical purposes of reliability analysis, the random functions are usually approximated by simplified models (jump processes or jump processes with intermittencies). The step-wise functions offer sufficiently accurate models provided that their parameters are adjusted to the character of the variable, to the properties of actions and materials.

In the case of time-dependent basic variables, the failure of a structure may occur due to two different events:

- first excess of a certain limit; and

- fatigue or accumulation of another type of material deterioration.

In some cases it is possible to reduce a time-dependent problem to a time-independent one. Such a simplification is possible when only one source of action is involved and when the failure is caused by the first excess of a certain limit. In this case the random function representing an action may be replaced by a probability distribution function describing the action's variability over the given time period (a required design working life), for which the probability of failure is to be assessed. The mean of the alternative distribution may be determined as the expected value of the maximal action over the given period, the standard deviation of the distribution corresponds to the variance of the maximal action's expected value.

An analogical procedure may be used in the case of failure due to fatigue. Using the Miner-Palmgren rule of accumulation of deterioration over a given period, a time-dependent fatigue strength with a corresponding probability distribution function may be expressed considering the usual dependence on the number of cycles and the stress.

However, in more complicated cases with more time-dependent basic variables, it is necessary to use appropriate models of time-dependent quantities and processes. The next section gives a concise description of some of the most important concepts applied in timevariant reliability analysis.

\subsection{Time-variant actions}

Consider a time-variant action (a random function) $Q$, for which one realisation of instantaneous values is shown in Figure 6.1. A probability density function of instantaneous values $Q$ denoted as $\varphi_{Q}(q)$ is indicated in Figure 6.1 on the right. The corresponding distribution function is $\Phi_{Q}(q)$. Suppose that the considered time period (a required design working life) $T$ is divided into small time intervals $\tau$ (for example one year). In the Eurocodes, such a time period is denoted as the reference period. For each time interval $\tau$ a maximal 
value (annual maximum) $Q_{i, \max }$ is determined. These maximum values form a new random variable of annual extremes $Q_{\max }$, which have a certain probability density function $\varphi_{Q \max }(q)$ (for example a Gumbel distribution) as shown in Figure 6.1 on the right. Note that symbol $q$ denotes here a particular value of $Q$ or $Q_{\max }$. A distribution function corresponding to the probability density function $\varphi_{Q \max }(q)$ is denoted as $\Phi_{Q \max }(q)$.
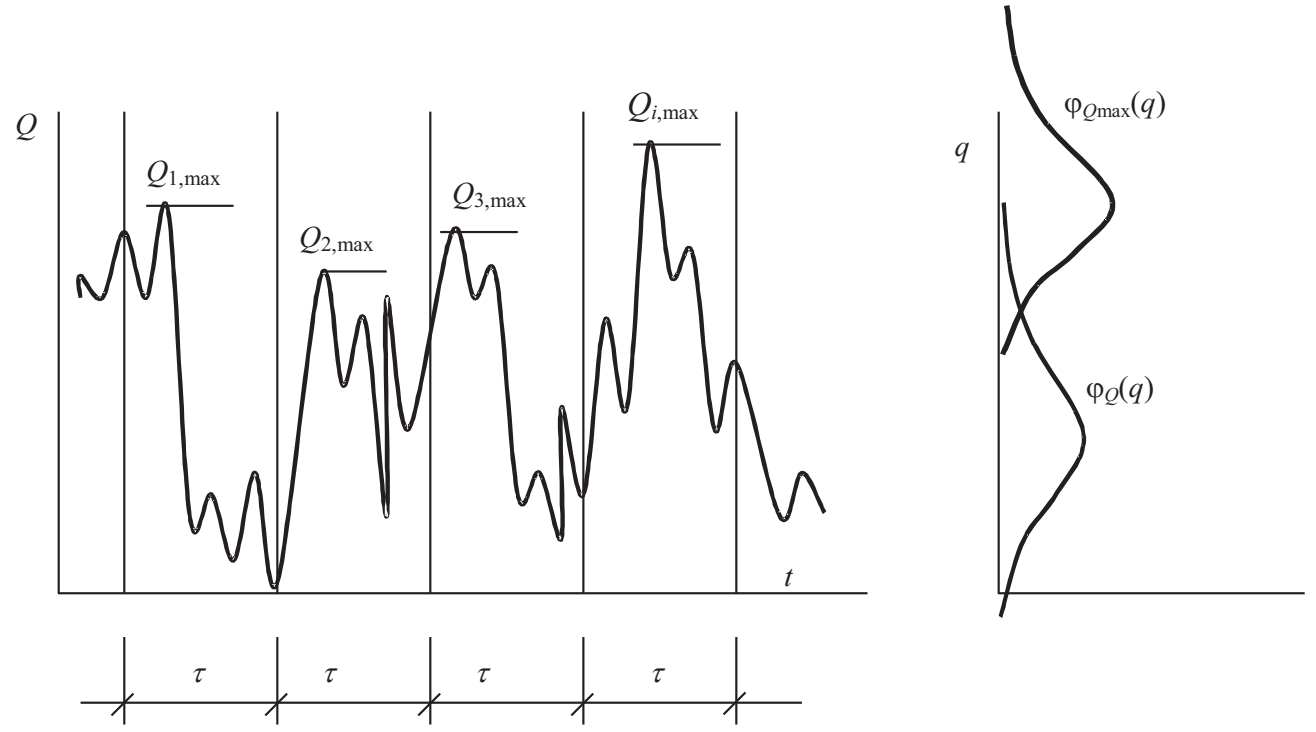

Figure 6.1. Time-dependent function of a random action $Q$.

The distribution functions $\varphi_{Q}(q)$ and $\varphi_{Q \max }(q)$ are shown in Figure 6.2. Obviously, the mean of the variable $Q_{\max }$ is greater than the mean of $Q$.

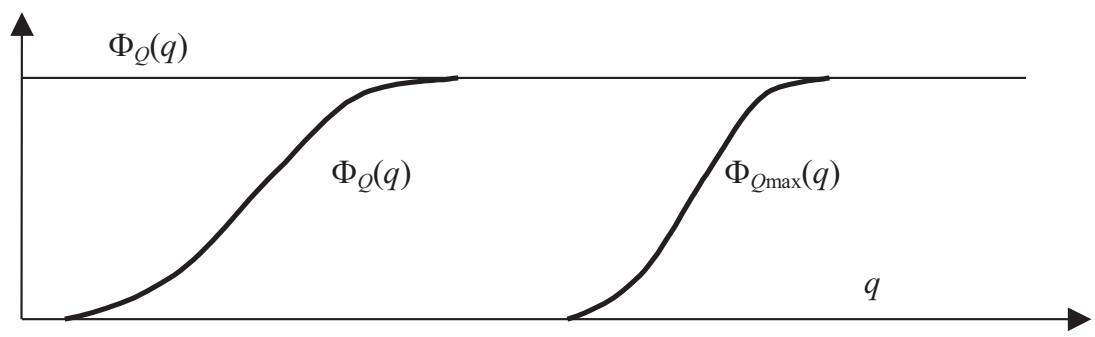

Figure 6.2. Distribution functions $\varphi_{Q}(q)$ and $\varphi_{Q \max }(q)$.

The definition of the characteristic value of an action is based on the distribution function $\Phi_{Q \max }(q)$, which is based on the specified reference period $\tau$ (for example one year). Note that the required design working life $T$ is usually 50 to 100 years. The characteristic value $q_{\mathrm{k}}$ is defined as the upper fractile of the distribution $\Phi_{Q \max }(q)$ corresponding to the specified probability $p$ (for example 0,02 ), so that it holds that

$$
\mathrm{P}\left(Q_{\max }>q_{\mathrm{k}}\right)=1-\Phi_{Q \max }\left(q_{\mathrm{k}}\right)=p
$$


Thus, the probability $p$ refers to the distribution $\Phi_{Q \max }(q)$ valid for a reference period $\tau$ and gives the probability that the characteristic value $q_{\mathrm{k}}$ will be exceeded by the maximal value $Q_{\max }$ assessed for the reference period $\tau$.

If the characteristic value $q_{\mathrm{k}}$ is exceeded by the maximum value $Q_{i \text {, max }}$ in each of the time periods $\tau$ with a probability $p$, then after a certain number of reference periods $\tau$ the characteristic value $q_{\mathrm{k}}$ will be exceeded with a probability equal to 1 . Such a time period is called the return period and is denoted as $T_{\text {ret }}$. It holds approximately that the number of reference periods is $1 / p$, and the mean return period is then

$$
T_{\text {ret }}=\tau / p=\tau /\left(1-\Phi_{Q \max }\left(q_{\mathrm{k}}\right)\right)
$$

The return period is commonly used to describe the characteristic value $q_{\mathrm{k}}$ or the significance of particular values of various climatic actions. A usual value of the return period fluctuates between 50 and 100 years, which corresponds to the required design working life $T$.

\section{Example 6.1}

The characteristic value of wind pressure $q_{\mathrm{k}}$ is defined for a reference period $\tau=1$ year and the probability of being exceeded by annual maxima $Q_{i, \max }$ is specified as $p=0,02$. The mean return period $T_{\text {ret }}$ is, according to relation (6.2), given as

$$
T_{\text {ret }}=\tau / p=1 / 0,02=50 \text { years }
$$

Therefore, it can be concluded that the characteristic value of wind pressure $q_{\mathrm{k}}$ has the mean return period of 50 years.

\subsection{Rectangular wave processes}

A rectangular wave renewal process (step-wise or jump process), which is an approximation of time-dependent variable actions, is an effective tool used to simplify timevariant reliability analysis. It is also applied when a combination of two variable actions $Q_{1}$ and $Q_{2}$ is analysed. A rectangular wave representation of a single variable action $Q$ is shown in Figure 6.3. Note that the time period is split into small time intervals of generally unequal duration. This is a generalisation of the Ferry Borges-Castanheta model for variable actions $Q$, in which the time intervals have a constant duration.

The step-wise function (jump or rectangular wave process) shown in Figure 6.3 is an approximation of a continuous random function describing a variable action $Q$ when no intermittencies in $Q(t)$ are considered. Furthermore, no distribution is considered for the time intervals in which new rectangular waves arrive. So, the process is fully characterised by the jump rate $\lambda$ and the distribution function of the amplitude of the rectangles. The jump rate $\lambda$ should be estimated considering the mean time interval $\mu_{\tau}$

$$
\lambda=1 / \mu_{\tau}
$$

It follows that the mean duration of pulses (rectangles) is $\mu_{\tau}=1 / \lambda$. In the analysis, it is further assumed that the renewals (rectangles) occur independently of each other. 


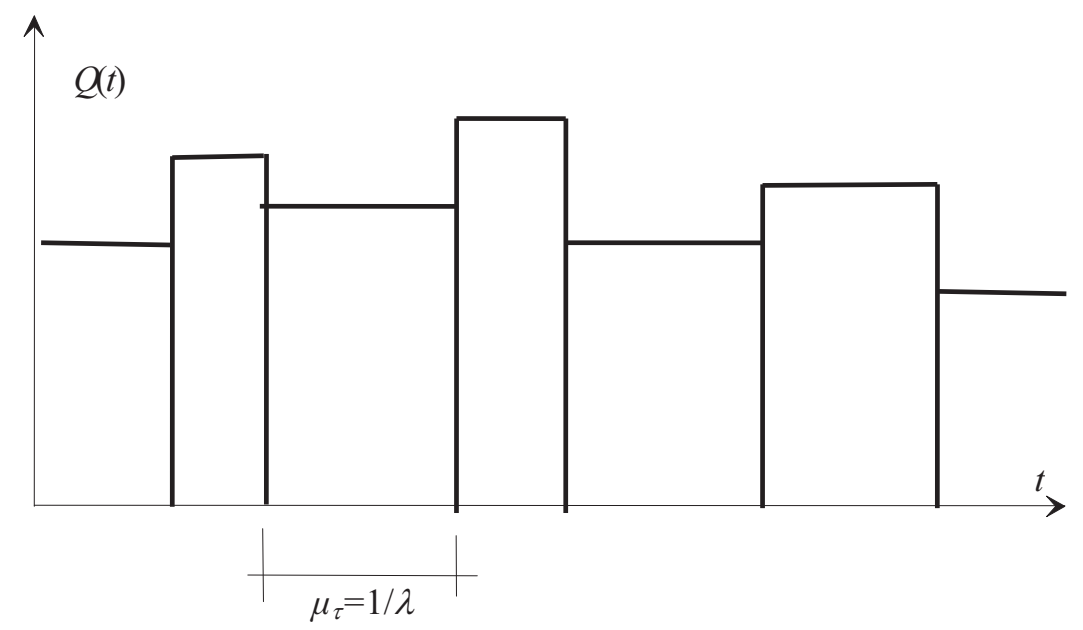

Figure 6.3. A rectangular wave process.

\section{Example 6.2}

Consider an imposed load in an office area. It is known that the long-term part of the load (furniture and other equipment) is changed on average every 5 years, thus $\mu_{\tau}=5$ years. It follows from equation (6.3) that the jump rate $\lambda$ is

$$
\lambda=1 / \mu_{\tau}=1 / 5=0,2[1 / \text { year }]
$$

\subsection{Rectangular wave processes with intermittencies}

A more refined model of variable action $Q(t)$ is a rectangular wave renewal process with intermittences, which is indicated in Figure 6.4. A detailed treatment of this model may be found in literature $[2,24,31,32]$. The following simplified description is limited to practical guidance useful for applications of some software products.

It is indicated in Figure 6.4 that there are intervals when the time-dependent variable load $Q(t)$ is "on" and intervals when the load $Q(t)$ is "off". Denote $\mu_{\tau}$ the mean of the renewal interval (shown in Figure 6.4) and $\mu_{\eta}$ the mean duration of the interval, when the load is "on". It is useful to define the so-called interarrival duration intensity of the variable load $Q(t)$ as the ratio

$$
\rho=\mu_{\eta} / \mu_{\tau}=\lambda \mu_{\eta}
$$

\section{Example 6.3}

Consider a wind load, which achieves significant values (storms) 10 times a year. The duration of each storm is about 8 hours. In a reliability analysis, the following parameters should be therefore considered: 


$$
\begin{gathered}
\mu_{\tau}=1 / 10 \text { [years], } \lambda=1 / \mu_{\tau}=10 \text { [1/year] } \\
\mu_{\tau}=1 / 10 \text { [years], } \mu_{\eta}=8 /(365 \times 24)=0,0009 \text { [year], } \rho=\mu_{\eta} / \mu_{\tau}=\lambda \mu_{\eta}=0,009
\end{gathered}
$$

Obviously, there might be a great uncertainty in the estimates of the parameters. Then it would be wise to determine the sensitivity of the reliability analysis to these parameters or to repeat the analysis using reasonable lower and upper estimates.

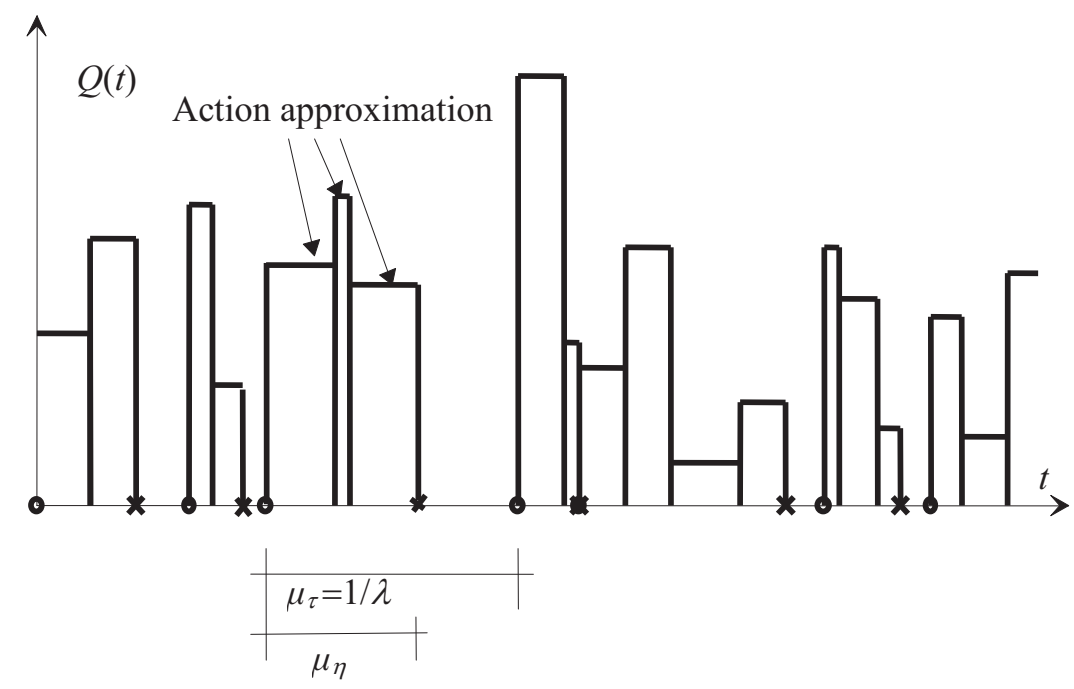

Figure 6.4. A rectangular wave process with intermittencies.

The interarrival duration intensity ratio $\rho$ of the variable load $Q(t)$ is applied in timevariant reliability analysis using the software package STRUREL.

\subsection{Combination of actions, Turkstra's rule}

The fundamental principle for the assessment of the effect of the combination of actions, according to the contemporary international documents [1,2], is based on the rule that the leading action (dominant) is not reduced and is considered at its full value, while the other accompanying (non-dominant actions) are reduced by factors $\psi_{0 i} \leq 1$. The principles of the assessment of the combination of actions will be demonstrated considering two random actions $Q_{1}(t)$ and $Q_{2}(t)$. It is assumed that both the actions may be described by step-wise (jump) functions as indicated in Figure (6.5) (a rectangular wave renewal process (step-wise functions) approximates the random function describing a time-variant action shown in Figure $6.1)$. $[22,24])$

To simplify the problem, it is further assumed that [2] (some terms are explained in

- $Q_{1}(t)$ and $Q_{2}(t)$ are stationary and ergodic processes,

- all intervals $\tau_{1}$ are equal,

- all intervals $\tau_{2}$ are equal,

$-\tau_{1} \geq \tau_{2}$ 
- if $r_{1}=T / \tau_{1}$ and $r_{2}=T / \tau_{2}$ where $T$ is the reference period, then $r_{1}$ and $r_{2} / r_{1}$ are integers,

$-Q_{1}(t)$ and $Q_{2}(t)$ are constant over the intervals $\tau_{1}$ and $\tau_{2}$,

- values $Q_{1}(t)$ and $Q_{2}(t)$ in two different intervals are mutually independent,

$-Q_{1}(t)$ and $Q_{2}(t)$ are independent.
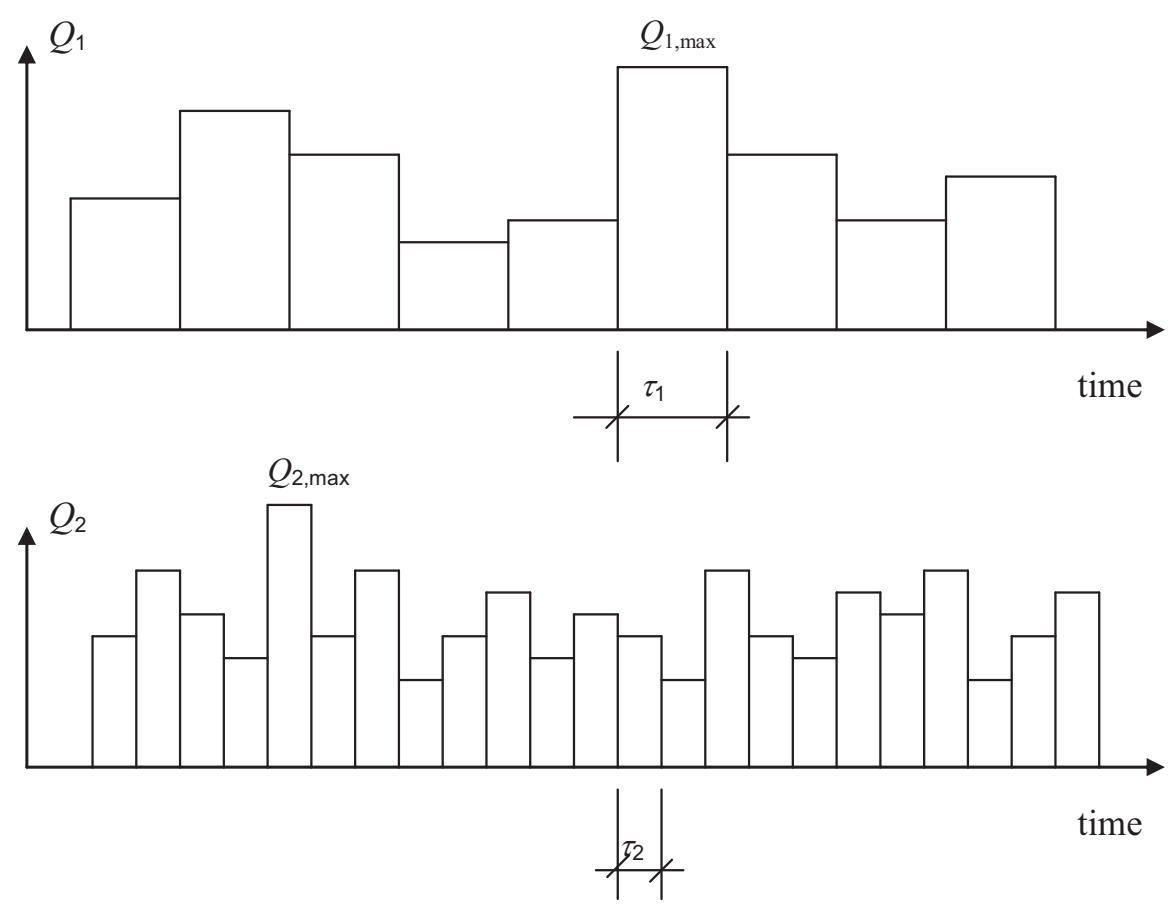

Figure 6.5. Approximation of random actions by step-wise functions.

For each of the actions $Q_{1}(t)$ and $Q_{2}(t)$ three different distribution functions are further determined:

1. Distribution of original values $Q$ is denoted as $\Phi_{Q}(q)$.

2. Distribution of maximum values $Q_{\max }$ determined for the reference period $T$

$$
\Phi_{Q \max }(q)=\left(\Phi_{Q}(q)\right)^{r}
$$

3. Distribution of the maximum value $Q_{1 \mathrm{c}}$ and $Q_{2 \mathrm{c}}$ over the interval $\tau_{1}$, obviously $Q_{1 \mathrm{c}}=Q_{1}$ and the distribution of $Q_{2 \mathrm{c}}$ is

$$
\Phi_{Q 2 \mathrm{c}}(q)=\left(\Phi_{Q}(q)\right)^{r 2 / r 1}
$$

Figure 6.6 shows the distribution functions considered for the action $Q_{2}$ for points 1 to 3 .

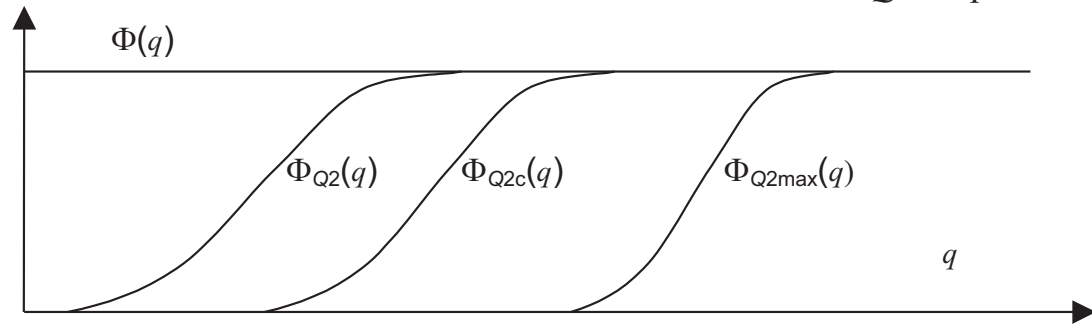

Figure 6.6. Distribution functions of the random action $Q_{2}$. 
Assume a linear relationship between the load effect $E_{\max }$ and the actions $Q_{1}$ and $Q_{2}$ :

$$
E=E\left(Q_{1}, Q_{2}\right)=a Q_{1}+b Q_{2}
$$

where $a$ and $b$ are appropriate factors depending on the structural and loading conditions. The maximum action effect $E_{\max }$ of the actions $Q_{1}$ and $Q_{2}$ during the whole reference period $T$ can be written as

$$
E_{\max }=\max E\left(Q_{1 \mathrm{c}}, Q_{2 \mathrm{c}}\right)
$$

where the maximum is considered for all intervals $\tau_{1}$ during the reference period $T$.

The resulting action effect may be approximately assessed as the maximum of the following values (the so-called Turkstra rule)

$$
\begin{aligned}
& E_{\max }=E\left(Q_{1 \max }, Q_{2 \mathrm{c}}\right) \text { where } Q_{1} \text { is considered as dominant } \\
& E_{\max }=E\left(Q_{1 \mathrm{c}}, Q_{2 \max }\right) \text { where } Q_{2} \text { is considered as dominant }
\end{aligned}
$$

which can be also written as

$$
E_{\max }=\max \left(E\left(Q_{1 \max }, Q_{2 \mathrm{c}}\right), E\left(Q_{1 \mathrm{c}}, Q_{2 \max }\right)\right)
$$

When the structure is designed by a probabilistic method, the actions according to relations (6.8) and (6.11) are considered as random actions having a distribution function as shown in Figure 6.6.

When the structure is designed by the partial factors method, the design value $E_{\text {max,d }}$ of the actions effect $E$ is determined from the relationship

$$
E_{\text {max }, \mathrm{d}}=\max \left(E\left(Q_{1 \text { max }, \mathrm{d}}, Q_{2 \mathrm{~cd}}\right), E\left(Q_{1 \mathrm{~cd}}, Q_{2 \text { max }, \mathrm{d}}\right)\right)
$$

where $Q_{1 \mathrm{max}, \mathrm{d}}=\gamma_{Q 1} Q_{1 \mathrm{k}}$ and $Q_{1 \mathrm{~cd}}=\gamma_{Q 1} \psi_{01} Q_{1 \mathrm{k}}$ and analogous relations hold for $Q_{2}$.

\subsection{Combination value of variable actions}

The relationships for combination factors $\psi_{0}$ may be derived in several ways. Equation (6.12), based on Turkstra's rule, is applied most often. The design combination value $Q_{\text {cd }}$ (it may refer to either the action $Q_{1}$ or $Q_{2}$ ) is selected as a fractile of the $Q_{\mathrm{c}}$ corresponding to the probability $\Phi\left(-0,4 \alpha_{E} \beta\right)$, thus

$$
\Phi_{Q}\left(Q_{\mathrm{cd}}\right)=\Phi\left(-0,4 \alpha_{E} \beta\right)
$$

where $\alpha_{E}=0,7$ is a weighting factor for the variable action $Q$. The combination factors $\psi_{0}$ follow (6.13) as

$$
\psi_{0}=\frac{Q_{c d}}{Q_{\max , d}}=\frac{\Phi_{Q c}^{-1}(\Phi(0,4 \times 0,7 \beta))}{\Phi_{\max }^{-1}(\Phi(0,7 \beta))}=\frac{\Phi_{\max }^{-1}\left(\Phi(0,4 \times 0,7 \beta)^{r}\right)}{\Phi_{\max }^{-1}(\Phi(0,7 \beta))}
$$

If the distribution $\Phi_{Q \max }(q)$ is approximated by the normal distribution, then equations (6.14) and (4.2) lead to the formula

$$
\psi_{0}=\frac{1+\Phi^{-1}\left(\Phi\left(0,4 \alpha_{E} \beta\right)^{r}\right) w}{1+\alpha_{E} \beta w}
$$

which may be approximated as indicated in [2]

$$
\psi_{0}=\frac{1+\left(0,4 \alpha_{E} \beta-0,7 \ln (r)\right) w}{1+\alpha_{E} \beta w}
$$

Note that $w$ denotes the coefficient of variation related to the extremes within the reference period $T$ (50 years). 
Considering the Gumbel distribution and equation (4.5) for its fractile, the relationship (6.14) becomes

$$
\psi_{0}=\frac{1-w\left\{0,45+0,78 \ln \left[-\ln \left(\Phi\left(0,4 \alpha_{E} \beta\right)\right)\right]+\ln (r)\right\}}{1-w\left\{0,45+0,78 \ln \left[-\ln \left(\Phi\left(\alpha_{E} \beta\right)\right)\right]\right\}}
$$

where $w$ denotes the coefficient of variation of the probability distribution $\Phi_{Q \max }(q)$ related to the reference period $T$ (50 years).

\section{Example 6.4}

Consider that an accompanying wind action $W$ is combined with a leading imposed action $Q$ assuming a required working life $T=50$ years. Considering Turkstra's rule, annual extremes of the wind $W$ should be combined with the 50 years extreme of the imposed load $Q$. For the leading action $Q$, it is assumed that it changes every 5 years, thus $r_{1}=T / \tau_{1}=50 / 5=10$, for the accompanying action - wind $W$ - annual extremes are considered, thus $r_{2}=T / \tau_{2}=$ 50/1. It follows that $r=r_{2} / r_{1}=5$. The coefficient of variation $w$ of the 50 years wind maximum is expected within the interval from 0,2 to 0,4 . The factor $\psi_{0}$, determined using the three relationships derived above, equations (6.15), (6.16) and (6.17), is shown in Figure 6.7 as a function of the coefficient of variation $w$.

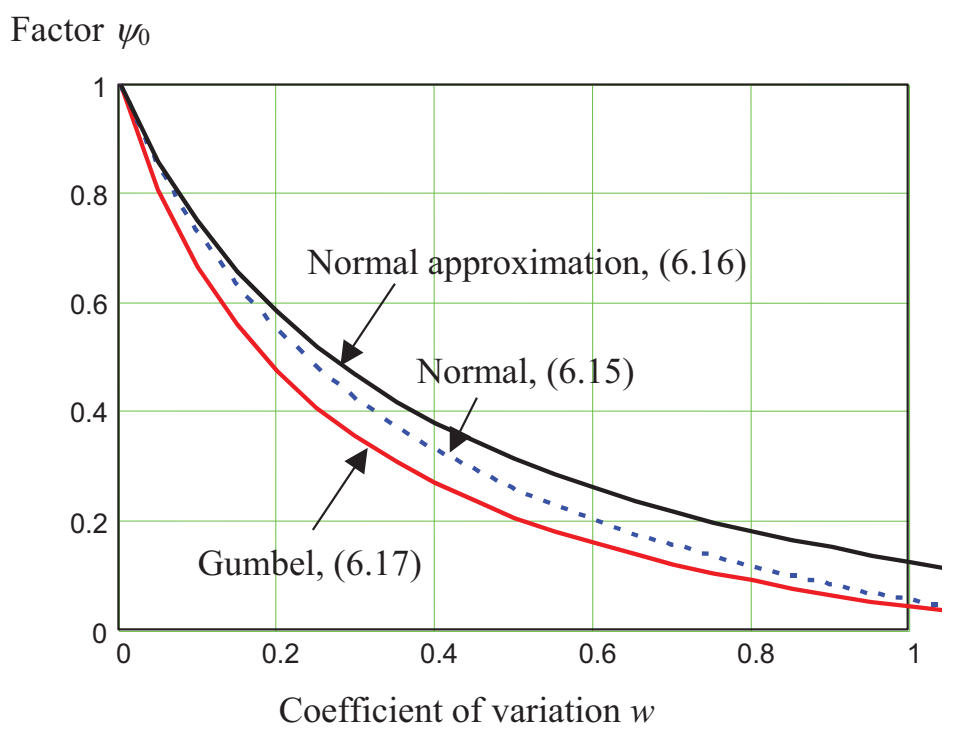

Figure 6.7. Factor $\psi_{0}$ for wind assuming $r=10$.

It follows from Figure 6.7 that the three expressions used to calculate $\psi_{0}$ yield similar results. With increasing coefficient of variation $w$ the factor $\psi_{0}$ decreases. It is interesting to note that for the coefficient of variation $w=0.3$ the factor $\psi_{0} \cong 0,4$, which is considerably lower than the value recommended in the present version of EN 1990 (where the value 0,7 is indicated). However, if the coefficient of variation $w=0,2$, then $\psi_{0}>0,4$.

Equations (6.15), (6.16) and (6.17) are also given in Appendix A to Eurocode 1 [1], where other combination values of the variable load $Q(t)$ for usual cases are recommended too. Besides the combination values $\psi_{0} Q_{\mathrm{k}}$ of random actions, yet other reductions of the characteristic values of random actions are applied in international documents: the frequent value $\psi_{1} Q_{\mathrm{k}}$ and the quasi-permanent value $\psi_{2} Q_{\mathrm{k}}$. Generally these reduced values are called 
the representative values of random actions. They are applied in various combinations of actions for ultimate limit states and serviceability limit states with regard to the type of design situation [1]. The frequent value $\psi_{1} Q_{\mathrm{k}}$ of random load $Q(t)$ is determined in such a way that the total period of time when this value is exceeded over a certain reference period is a small part $(0,01)$ of this interval. The quasi-permanent value $\psi_{2} Q_{\mathrm{k}}$ of the random load $Q(t)$ is defined as the value, which is exceeded during a considerable part $(0,5)$ of the reference period (this is how it differs from the frequent value).

The relationship between the characteristic value $Q_{\mathrm{k}}$, combination value $\psi_{0} Q_{\mathrm{k}}$, frequent value $\psi_{1} Q_{\mathrm{k}}$ and quasi-permanent value $\psi_{2} Q_{\mathrm{k}}$ of a random action is shown in a schematic way in Figure 6.8. Relative values of the representative values indicated in Figure 6.8 correspond approximately to the values recommended in Eurocode $1: \psi_{0} \approx 0,7, \psi_{1} \approx 0,5$ and $\psi_{2} \approx 0,3$. Detailed tables of recommended values for typical variable actions $Q$ are given in Eurocode 1 [1]. The described models and their further improvements can be found in recent materials $[16,27]$.

\section{Instantaneous value $Q$}

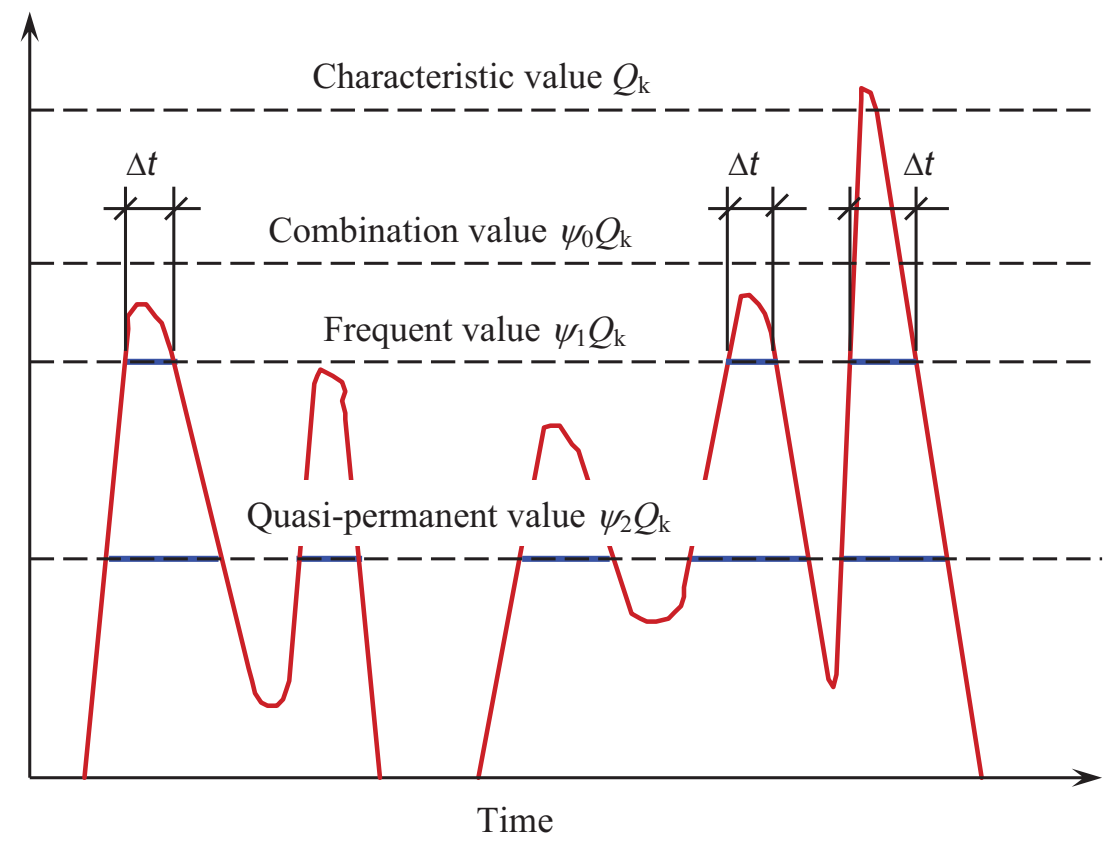

Figure 6.8. Representative values of random actions $Q$.

\subsection{Frequent and quasi-permanent values}

The frequent and quasi-permanent values, $Q_{1}$ and $Q_{2}$, of variable actions $Q$ are defined through the relative duration of exceeding $Q_{1}, \eta_{1}=0,01$, and relative duration of exceeding $Q_{2}, \eta_{2}=0,5$. As mentioned above the relative duration is defined as (see Figure 6.8)

$$
\eta_{1(2)}=\sum \Delta t_{i} / T
$$

where $T$ denotes a certain reference period. Let us denote the distribution function of non-zero $Q$ variable action $\Phi_{Q^{\prime}}(Q)$ Probability of exceeding $Q_{1}$ and $Q_{2}$ may be written as

$$
p_{1(2)}=P\left(Q^{\prime}>Q_{1(2)}\right)=1-\Phi_{Q^{\prime}}\left(Q_{1(2)}\right)
$$


If the probability of non-zero $Q$ is $q$, then the relative duration of exceeding $Q_{1}$ and $Q_{2}$ may be written as $\eta_{1(2)}=p_{1(2)} q$ and the probability as $p_{1(2)}=\eta_{1(2)} / q$. It follows then from equation (6.18) that the frequent and quasi-permanent values $Q_{1}$ and $Q_{2}$ may be expressed as

$$
Q_{1,2}=\Phi_{Q^{\prime}}^{-1}\left(1-\frac{\eta_{1(2)}}{q}\right)
$$

The corresponding factors $\psi$ are then given as

$$
\psi_{1}=Q_{1} / Q_{k}, \quad \psi_{2}=Q_{2} / Q_{k}
$$
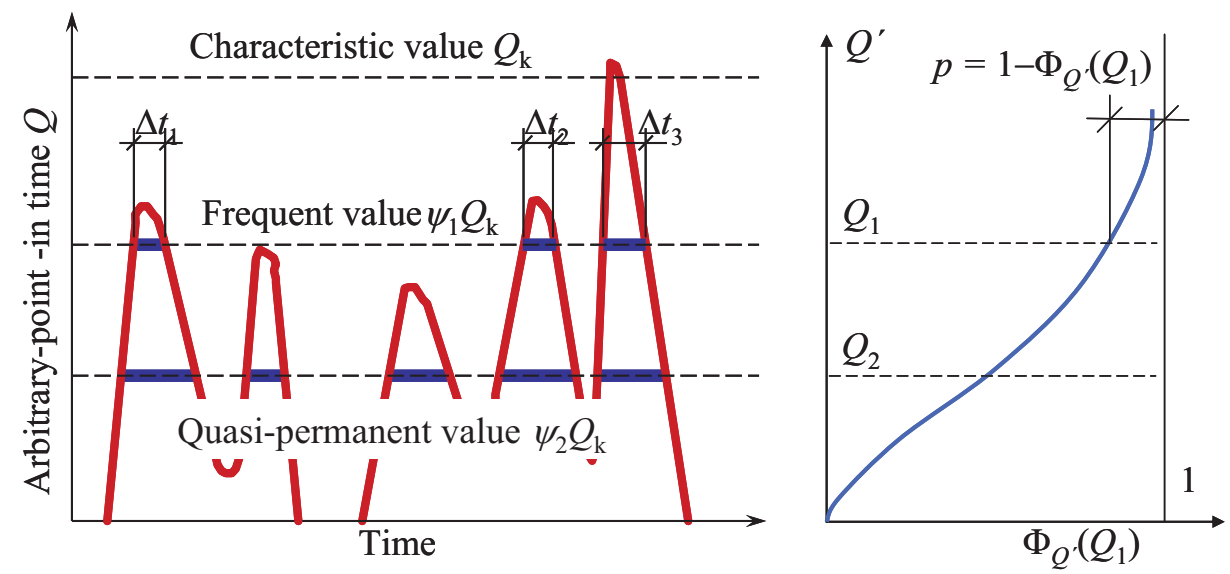

Figure 6.9. Frequent and quasi-permanent values of a variable action $Q$.

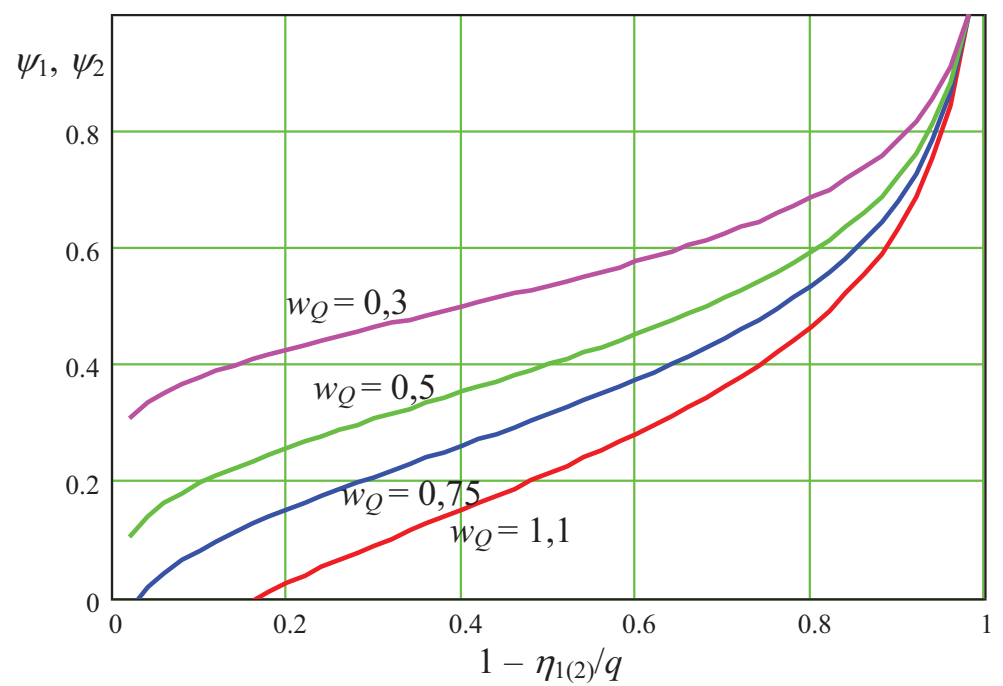

Figure 6.10. Factors $\psi_{1}$ and $\psi_{2}$ for frequent and quasi-permanent values of a variable action $Q$. 
Assuming Gumbel distribution, equations (6.19) and (6.20) lead to the expression

$$
\psi_{1(2)}=\frac{1-w\left\{0,45+0,78 \ln \left[-\ln \left(\Phi\left(1-\frac{\eta_{1(2)}}{q}\right)\right)\right]\right\}}{1-w\{0,45+0,78 \ln [-\ln (\Phi(0,98))]\}}
$$

In equation (6.21) the characteristic value $Q_{\mathrm{k}}$ in denominator is considered as 0,98 fractile of annual extremes ("point in time") distribution of $Q$. Figure 6.10 shows both factors $\psi_{1}$ and $\psi_{2}$ for frequent and quasi-permanent values of a variable action $Q$ determined from equation (6.21). Note that the coefficient of variation $w_{Q}$ relates to the annual extremes distribution.

\section{Example 6.5}

Consider a wind action having 10 storms a year, each of 8 hours duration. Thus the probability $q=10 /(365 \times 3)=0,009$ and $\left(1-\eta_{1} / q\right)=0,011$. Considering $w_{Q}=0,5$ it follows from Figure 6.10 that $\psi_{1}=0,2$, the value recommended in EN 1990 [1]. It follows that $\psi_{2}=$ 0,0 .

\subsection{Deterioration of structural members}

Material deterioration due to unfavourable effects of external environment (for example of humidity, carbon dioxide and chlorides) represent important time-dependent phenomena that may significantly affect structural reliability and durability. Due to a number of unknown factors these deterioration processes are usually quite variable. A probabilistic approach to their analysis is thus most desirable.

The examined resulting quantity, describing the degradation of material, is in fact the effect of external influences and is thus denominated as $E(t)$, where $t$ indicates time. The assumption is made that it is a monotonously increasing function of time (for example the carbonation depth). To preserve the assumed structural properties it is required that this quantity does not exceed the general time-dependent structural resistance $R(t)$ (for example the thickness of the reinforcement cover layer), which is supposed to be a monotonously decreasing function of time $t$. Figure 6.11 shows an expected shape of the functions $E(t)$ and $R(t)$.

The basic condition of an acceptable (satisfactory or safe) state is thus the inequality

$$
E(t)<R(t)
$$

which is in fact a generalisation of inequality (5.1). Therefore, the next steps follow the general procedure described in chapter 5. For monotonous functions $E(t)$ and $R(t)$ condition (6.22) leads to the problem of the first excess of a given limit (see the types of failure for the time-dependent events given in Section 6.1).

Figure 6.11 further indicates that the random quantities $E(t)$ and $R(t)$ are described by probability density functions $\varphi_{E}(x ; t)$ and $\varphi_{R}(x ; t)$ and by the distribution functions $\Phi_{E}(x ; t)$ and $\Phi_{R}(x ; t)$, which are functions - first, of a realisation $x$ of the observed random variable and secondly, of a non-random time $t$ (to distinguish the nature of these two quantities we used a semi-colon to separate them). The time-dependent probability distributions depend on the moment parameters $\mu_{E}(t), \sigma_{E}(t), \alpha_{E}(t), \mu_{R}(t), \sigma_{R}(t)$ and $\alpha_{R}(t)$, which, obviously, are generally functions of time $t$. 


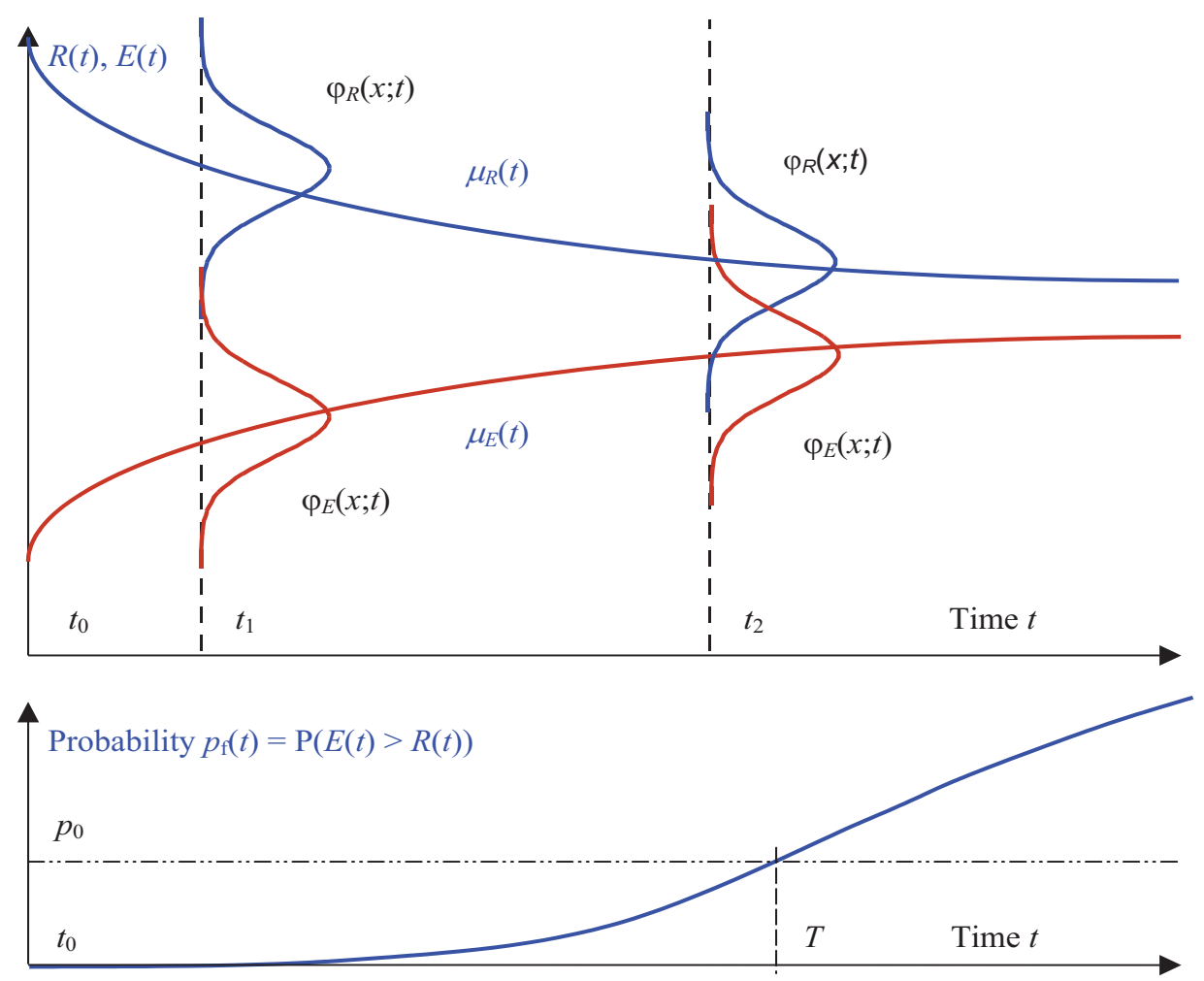

Figure 6.11. Time-variant functions $E(t)$ and $R(t)$.

Considering the equations (5.3) and (5.21), the time-dependent probability of failure $p_{\mathrm{f}}(t)$, i.e. the probability that the condition (6.22) will be violated, may be written in the form

$$
p_{\mathrm{f}}(t)=P(E(t)>R(t))=\int_{-\infty}^{\infty} \Phi_{R}(x ; t) \varphi_{E}(x ; t) \mathrm{d} x
$$

The structural lifetime $T$ may now be defined as the time during which the condition (6.22) is violated only with a given probability $p_{\mathrm{o}}$ (for example 0,05 ), i.e. the time for which it holds

$$
p_{\mathrm{f}}(T)=p_{0}
$$

Assuming the monotonous functions $E(t)$ and $R(t)$, the probability $p_{\mathrm{f}}(t)$ is also monotonous and its inverse function $p_{\mathrm{f}}^{-1}(p)$ may be defined. Then the lifetime $T$ may be written as

$$
T=p_{\mathrm{f}}^{-1}\left(p_{0}\right)
$$

The structural lifetime $T$ is thus linked to the probability $p_{0}$ that the observed functional property of the structure will not be satisfied (a failure will occur). When the lifetime $T$ is exceeded, also the probability $p_{\mathrm{f}}$ of failure exceeds the value $p_{0}$. 
The general procedure is illustrated on carbonation and loss of protecting properties of concrete. In this case the condition (6.22) may be simplified to an inequality between the neutralisation depth $d(t)$ and the time independent cover layer thickness $c$ (see Figure 6.12).

$$
d(t)<c
$$

Random behaviour of both quantities $d(t)$ and $c$ may be described by three-parameter log-normal distributions.

Time-dependence of the carbonation depth $d(t)$ is usually approximated by a semiempirical relationship for the square root of the effective time $t_{\text {eff }}$

$$
d(t)=A \sqrt{ } t_{\text {eff }}
$$

where $A$ is the coefficient of carbonation and the effective time $t_{\text {eff }}$ is defined as the weighted average of rainy, humid and dry periods. According to CEB (see also paper [35]) the $t_{\mathrm{eff}}=t^{1-2 n}$ where $n$ is a parameter of climatic conditions ( 0 for inner conditions, 0,3 for exposed, unsheltered outer conditions).

\section{Example 6.6}

From an extensive study of cooling towers [35] in unprotected outer conditions these values for the mean $\mu_{\mathrm{d}}$, coefficient of variation $w_{\mathrm{d}}$ and skewness $\alpha_{\mathrm{d}}$ were assessed

$$
\mu_{\mathrm{d}}=4 t^{0,2} \mathrm{~mm}, w_{\mathrm{d}}=0,35, \alpha_{\mathrm{d}}=0,5
$$

where $t$ is time expressed in years. The cover thickness $c$ is characterised as follows

$$
\mu_{\mathrm{c}}=20,25 \text { and } 30 \mathrm{~mm}, \sigma_{\mathrm{c}}=10 \mathrm{~mm}, \alpha_{\mathrm{c}}=1,0
$$

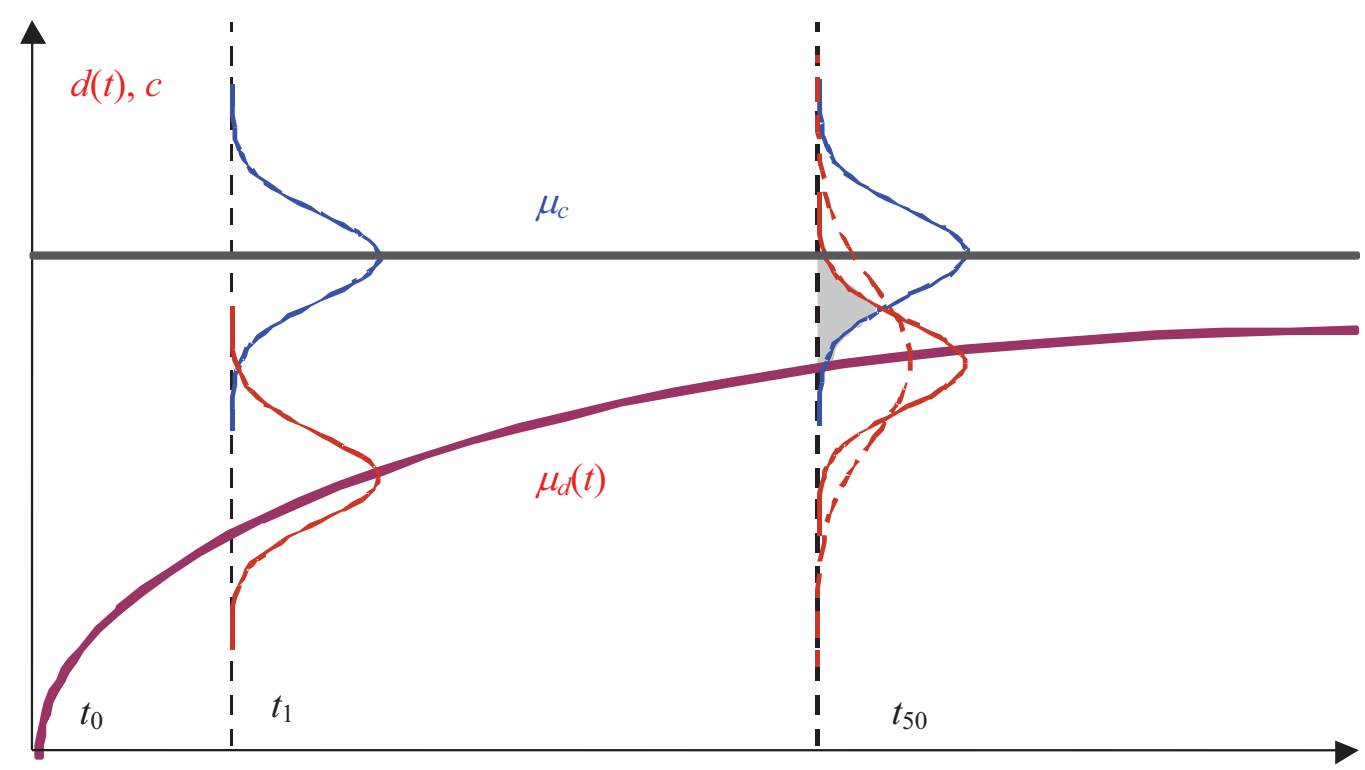

Time $t$

Figure 6.12. Carbonation depth $d(t)$ and thickness of cover layer $c$. 
The probability of failure $p_{\mathrm{f}}(t)$ for the parameters given in equations (6.28) and (6.29) is shown in Figure 6.13 that can be used for estimating $p_{\mathrm{f}}$ or $T$ given by equations (6.24) and (6.25). If a probability $p_{\mathrm{f}}=0,01$ is required, then a cover layer $c$ having the mean $\mu_{c}=25 \mathrm{~mm}$ ensures a working life of about 15 years only, the mean $\mu_{c}=30 \mathrm{~mm}$ ensures about 100 years.

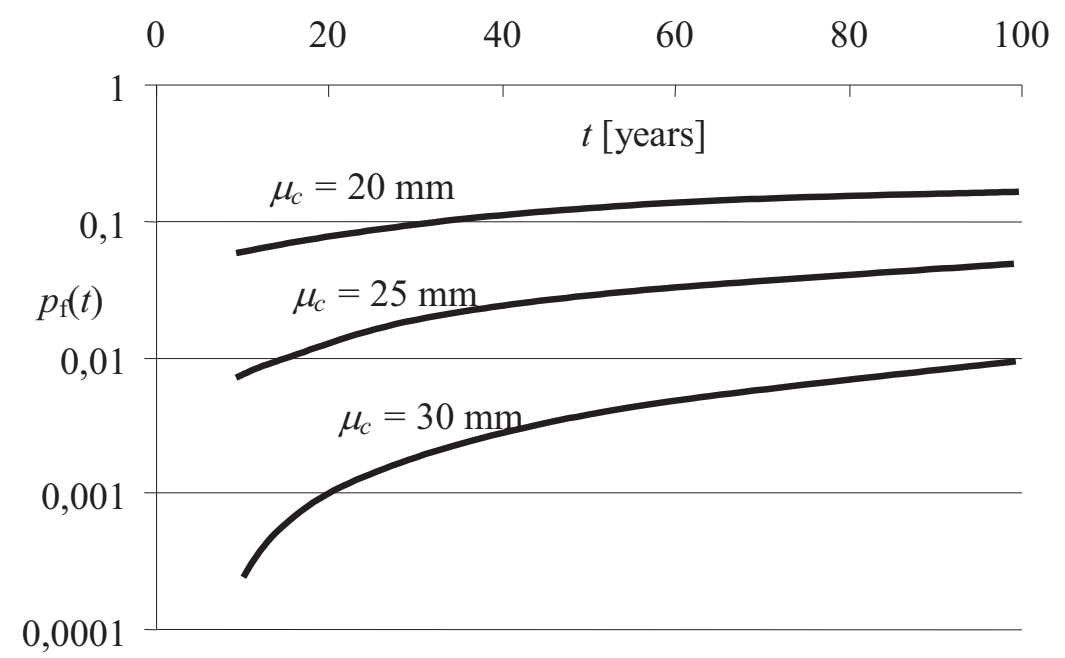

Figure 6.13. Probability of failure $p_{\mathrm{f}}(t)$ for parameters given in (6.28) and (6.29).

What is, however, the optimum probability of failure $p_{\mathrm{f}}$ ? This important question may be resolved using methods of probabilistic optimisation generally described in section 5.10.

The whole procedure of probabilistic cost optimisation is based on the concept of an objective function giving the total cost $C_{\text {tot }}$ as the sum of initial costs $C_{0}$, marginal costs $c C_{1}$, where $c$ denotes the thickness of cover layer and $C_{1}$ the cost per unit of thickness $c$, and costs due to failure $p_{\mathrm{f}}(t ; c) C_{\mathrm{f}}$, where $p_{\mathrm{f}}(t ; c)$ is the probability of failure in time $t$ for a thickness $c$. Thus, $C_{\text {tot }}$ can be written as

$$
C_{\text {tot }}=C_{0}+c C_{1}+p_{\mathrm{f}}(t ; c) C_{\mathrm{f}}
$$

If $C_{0}, C_{\mathrm{f}}$ and $\mu_{c}$ are independent of $c$, then the derivative $\partial p_{\mathrm{f}}(t ; c) / \partial c$ may be substituted by the expression $\partial p_{\mathrm{f}}(t ; c) / \partial \mu_{c}$ and the necessary condition for the minimum total costs is

$$
C_{1} / C_{\mathrm{f}}=-\frac{\partial p_{\mathrm{f}}(t ; c)}{\partial \mu_{c}}
$$

Relationships (6.30) and (6.31) are accepted for developing a special-purpose Mathcad sheet applied in the following example. 


\section{Example 6.7}

An example of cost optimisation is illustrated in Figure 6.14, which shows partial cost $c+p_{\mathrm{f}}(t ; c) C_{\mathrm{f}} / C_{1}$. This partial cost, which attains the minimum for the same mean $\mu_{c}$ as $C_{\text {tot }}$, was determined using a special-purpose software tool for selected values of the cost ratio $C_{\mathrm{f}} / C_{1}$, design durability of 50 years and data from Example 6.6 (i.e. for $\mu_{\mathrm{d}}=4 t^{0,2} \mathrm{~mm}, w_{\mathrm{d}}=$ $\left.0,35, \alpha_{\mathrm{d}}=0,5, \sigma_{\mathrm{c}}=10 \mathrm{~mm}, \alpha_{\mathrm{c}}=1,0\right)$.

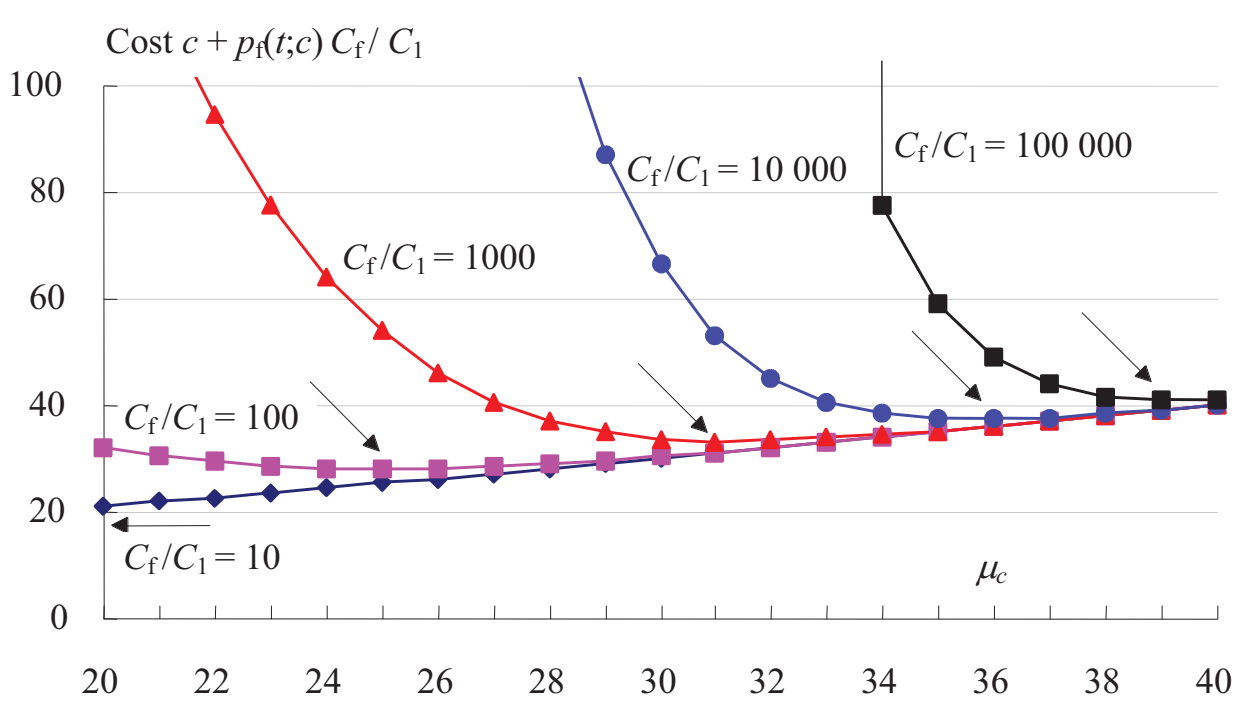

Figure 6.14. Cost $c+p_{\mathrm{f}}(t ; c) C_{\mathrm{f}} / C_{1}$ for parameters given in equations (6.28) and (6.29).

It follows from Figure 6.14 that it is possible to assess the optimal thickness of cover layer in respect of the ratio of costs $C_{\mathrm{f}} / C_{1}$; the minimum cost values are indicated by arrows.

Recent studies [35] seem to indicate that the carbonation depth $d(t)$ may be well described by Gamma distribution having the coefficient of variation

$$
w(t)=\alpha(t) / 2=0,1 t^{0,5}
$$

Available statistical data for the concrete cover also have a positive skewness, which is approximately equal to the coefficient of variation $\alpha \approx w$. Two types of distribution are therefore applicable: the three-parameter log-normal or the beta distribution. As the concrete cover is obviously a both sided limited random variable, the $\operatorname{Beta}$ distribution $\operatorname{Beta}(\mu, \sigma, \alpha, b)$ can be used. A developed MATHCAD sheet provides a simple tool to evaluate the reliability of reinforcement protection as shown by the following example.

\section{Example 6.8}

Another example of cost optimisation is illustrated in Figure 6.15, which shows again the partial cost $c+p_{\mathrm{f}}(t ; c) C_{\mathrm{f}} / C_{1}$. This partial cost, which attains the minimum for the same mean $\mu_{c}$ as the total $\operatorname{cost} C_{\text {tot }}$, was determined using a special-purpose MATHCAD sheet for 
selected values of the ratio $C_{\mathrm{f}} / C_{1}$, design durability of 50 years and modified data from Example 6.6:

$$
\begin{gathered}
\mu_{d}=4 t^{0,2} \mathrm{~mm}, w_{d}(t)=\alpha_{d}(t) / 2=0,1 t^{0,5} \\
w_{\mathrm{c}}=0,4 \mathrm{~mm}, \alpha_{\mathrm{c}}=0,5
\end{gathered}
$$

Figure 6.15 shows the partial cost $c+p_{\mathrm{f}}(t ; c) C_{\mathrm{f}} / C_{1}$ versus the concrete cover $c$ assuming a time $t=50$ years, a gamma distribution for the carbonation depth $d(t)$ and a beta distribution for the concrete cover $c$.

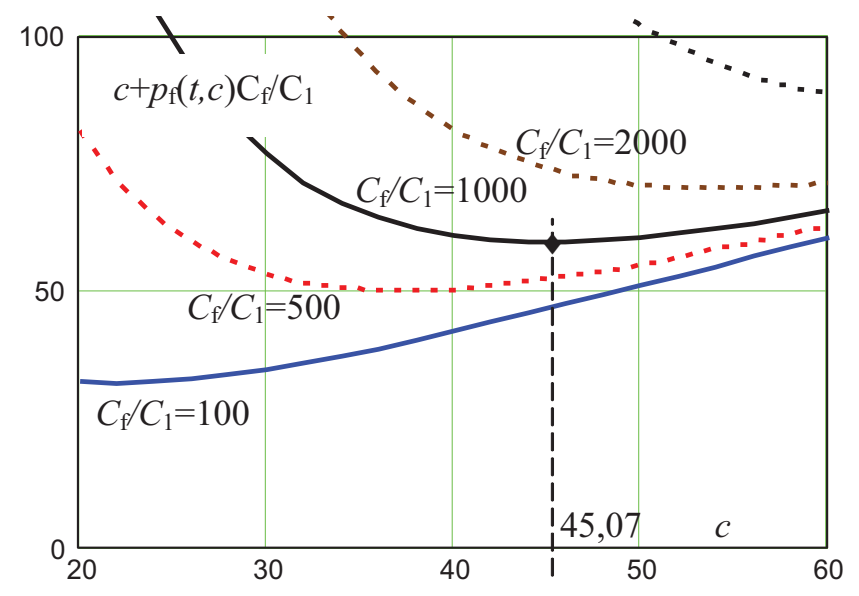

Figure 6.15. The partial $\operatorname{cost} c+p_{\mathrm{f}}(t ; c) C_{\mathrm{f}} / C_{1}$ versus the concrete cover $c$ for $t=50$ years.

The developed MATHCAD sheet also calculates precise values of the optimum concrete cover. For example assuming the cost ratio $C_{\mathrm{f}} / C_{1}=1000$ the optimum concrete cover is estimated as $45,07 \mathrm{~mm}$ (see Figure 6.15). An open task is to assess the cost ratio $C_{\mathrm{f}} / C_{1}$. 


\subsection{Introduction}

The following reliability analysis of a reinforced concrete column is an extension of a previous study by Holický \& Vrouwenvelder [31] based on the recent theoretical development of time-variant reliability analysis [32]. The study is a part of an extensive research activity on Probabilistic Model Code [27] supervised by the Joint Committee for Structural Safety (JCSS). The JCSS aims at providing a standardised set of statistical models for loads and structural properties, which will reflect the present state of knowledge.

Simpler stochastic models considered beforehand, when time-variant variables were described by the simple jump process [31], are superseded here by the jump process with intermittencies developed by Rackwitz [40]. In addition, some minor improvements in the deterministic design of the column were accepted.

The variable actions (short- and long-term imposed loads and wind) are considered as stationary and ergodic random processes described by square wave (jump) sequences. Each process is characterised by a jump rate $\lambda$ and an interarrival duration intensity $\rho$, both related to one year. A total of 12 study cases of the concrete column, designed according to the newly developing Eurocode EN 1990 [1], EN 1991 and EN 1992, are analysed using the software COMREL [24] (Reliability Consulting Programs (RCPs) (1997)).

\subsection{Model structure}

A model multi-storey structure considered in this study is schematically shown in Figure 7.1. It is assumed that each frame in the transversal direction of the structure may be considered as an unbraced sway frame. These frames consist of four columns at a constant transverse distance $a_{1}$; in the longitudinal direction of the structure the frames are located within a constant distance $a_{2}$ (see Figure 7.1).

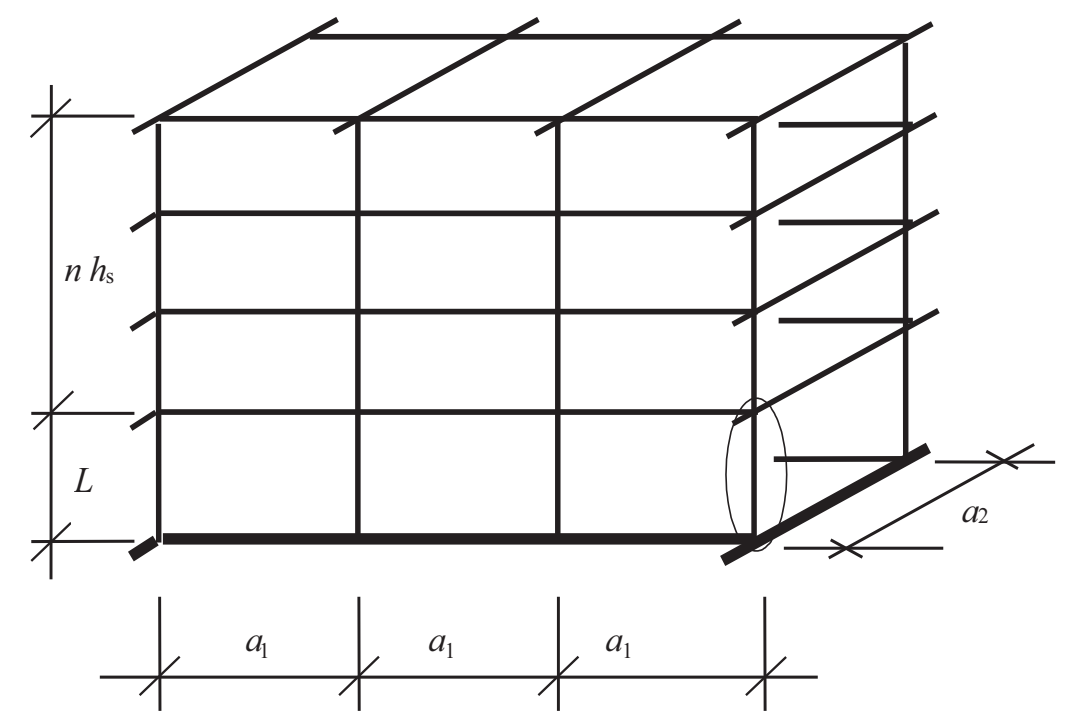

Figure 7.1. Transverse frame of a multi-storey structure. 
In the following reliability analysis the edge, fully clamped column of an internal transversal frame having the height $L$ (see Figure 7.1) and rectangular cross-section $b \times h$ is considered. The cross-section height $h$ is twice (in one study case, three times) the width $b$. Considering different structural arrangements, a total of 12 study cases indicated in Table 7.1 are analysed. Further, it is assumed that the storey height above the considered column is $h_{\mathrm{s}}=$ $3 \mathrm{~m}$, permanent load is determined as the weight of a reinforced concrete floor of a uniform equivalent thickness of $0,30 \mathrm{~m}$ (including the weight of the slab, columns, beams, floor and cladding).

Table 7.1. Study cases of a reinforced column.

\begin{tabular}{|c|c|c|c|c|c|}
\hline Case & $\begin{array}{c}\text { Number of } \\
\text { storeys } \\
n\end{array}$ & $\begin{array}{c}\text { Height of the } \\
\text { column } \\
L[\mathrm{~m}]\end{array}$ & $\begin{array}{c}\text { Transversal } \\
\text { distance } \\
a_{1}[\mathrm{~m}]\end{array}$ & $\begin{array}{c}\text { Longitudinal } \\
\text { distance } \\
a_{2}[\mathrm{~m}]\end{array}$ & $\begin{array}{c}\text { Cross-section } \\
\text { dimension } \\
b \times h[\mathrm{~m} \times \mathrm{m}]\end{array}$ \\
\hline 1 & 10 & 6 & 5 & 5 & $0,35 \times 0,70$ \\
\hline 2 & 10 & 3 & 5 & 5 & $0,30 \times 0,60$ \\
\hline 3 & 10 & 9 & 5 & 5 & $0,35 \times 0,70$ \\
\hline 4 & 10 & 12 & 5 & 5 & $0,45 \times 0,90$ \\
\hline 5 & 10 & 6 & 4 & 5 & $0,30 \times 0,60$ \\
\hline 6 & 10 & 6 & 7 & 5 & $0,35 \times 0,70$ \\
\hline 7 & 10 & 6 & 5 & 4 & $0,30 \times 0,60$ \\
\hline 8 & 10 & 6 & 5 & 7 & $0,40 \times 0,80$ \\
\hline 9 & 1 & 6 & 5 & 5 & $0,25 \times 0,50$ \\
\hline 10 & 3 & 6 & 5 & 5 & $0,25 \times 0,50$ \\
\hline 11 & 20 & 6 & 5 & 5 & $0,45 \times 0,90$ \\
\hline 12 & 10 & 6 & 5 & 5 & $0,25 \times 0,75$ \\
\hline
\end{tabular}

The effects of actions considered in the analysis of a built-in column consist of the axial force and bending moment, denoted again by $N$ and $M$ with appropriate subscripts. In the design calculation, the axial force and bending moment are represented by the design values $N_{\mathrm{d}}$ and $M_{\mathrm{d}}$ respectively. The maximum design axial force $N_{\mathrm{d} \text {,max }}$ is given as

$$
N_{\mathrm{d}, \max }=\gamma_{G} N_{\mathrm{W}, \mathrm{k}}+\gamma_{Q} \max \left\{N_{\mathrm{imp}, \mathrm{k}}+\psi_{0} N_{\text {wind,k }} ; N_{\text {wind,k }}+\psi_{0} N_{\text {imp,k }}\right\}
$$

where $\gamma_{\mathrm{G}}=1,35$ is the partial factor for permanent actions, $\gamma_{\mathrm{Q}}=1,50$ is the partial factor for variable actions, $\psi_{0}$ is the factor for combination value, $N_{\mathrm{W}, \mathrm{k}}$ is the characteristic value of the axial force due to self-weight, $N_{\text {imp,k }}$ is the characteristic value due to imposed load and $N_{\text {wind,k }}$ is the characteristic value due to wind (positive values are assumed for compressive forces). Taking into account the arrangement of the structure indicated in Figure 7.1, the characteristic value due to self-weight of $n$ floors and one roof is given as

$$
N_{\mathrm{W}, \mathrm{k}}=(n+1) a_{1} a_{2} t \rho_{\mathrm{c}} / 2
$$

where $\rho_{\mathrm{c}}$ is the weight of concrete per unit volume considered as $0,024 \mathrm{MN} / \mathrm{m}^{3} . N_{\text {imp, }}$ is the characteristic value of imposed load from $n$ storeys given as

$$
N_{\text {imp, } \mathrm{k}}=n a_{1} a_{2} p_{\text {imp }} \alpha_{\mathrm{n}} / 2
$$

Where, according to Eurocode 1 EN 1991-1-1 (2004), the reduction factor:

$\alpha_{\mathrm{n}}=\left[2+(n-2) \psi_{0}\right] / n$; here $n$ is the number of storeys $(>2)$ and $\psi_{0}$ is the load combination factor given in EN 1990 [1]. Choosing the category B (Public Building) the characteristic value of floor imposed load $p_{\text {imp,k }}$ equals $3 \mathrm{kN} / \mathrm{m}^{2}$. $N_{\text {wind,k }}$ is the wind resulting from a pressure $C_{\mathrm{p}} G p_{\text {wind,k }}$ on a vertical area equal to $\left(L+n h_{\mathrm{s}}\right) a_{2} ;$ multiplication by the height $\left(L+n h_{\mathrm{s}}\right) / 2$ 
gives the overturning moment. This moment is assumed to be balanced by the normal forces in the two outer columns, so:

$$
N_{\text {wind, } \mathrm{k}}=\left((1 / 2)\left(L+n h_{\mathrm{s}}\right)^{2} a_{2} C_{\mathrm{p}} G p_{\text {wind }, \mathrm{k}}-4 M_{\mathrm{d} 0}\right) /\left(3 a_{1}\right)
$$

where $M_{\mathrm{d} 0}$ is the first order bending moment at the bottom of one column. The characteristic value of the wind action is considered assuming the return period of 50 years as $p_{\text {wind,k }}=0,5$ $\mathrm{kN} / \mathrm{m}^{2}$; the gust (exposure) factor $G=2,5$ and the shape factor $C_{\mathrm{p}}=0,8+0,5=1,3$ (EN 1991$1-4(2004))$. moment is

In accordance with EN 1992-1: Eurocode 2 (2004) the design value $M_{\mathrm{d}}$ of the bending

$$
M_{\mathrm{d}}=M_{\mathrm{d} 0}+N_{\mathrm{d}}\left(e_{\mathrm{a}}+e_{2}\right)=N_{\mathrm{d}}\left(e_{0}+e_{\mathrm{a}}+e_{2}\right)
$$

where $e_{0}=M_{\mathrm{d} 0} / N_{\mathrm{d}}$ is the first order eccentricity, $e_{\mathrm{a}}$ is the additional eccentricity taking into account geometric imperfections and $e_{2}$ is the second order eccentricity due to deformations of the column. It is assumed that the first order moment $M_{\mathrm{d} 0}$ is caused only by wind action and is approximately given as

$$
M_{\mathrm{d} 0}=L\left[\gamma_{Q} C_{\mathrm{p}} G p_{\text {wind,k }}\left(L+n h_{\mathrm{s}}\right) a_{2}\right] / 8
$$

where $L$ denotes the column height (see Figure 7.1). The eccentricities $e_{\mathrm{a}}$ and $e_{2}$ are determined according to Eurocode 2 (EN 1992 (2004)) as

$$
\begin{aligned}
& e_{\mathrm{a}}=1,12 L /(2 \times 200)=0,0028 L \\
& e_{2}=0,1 K_{1} l_{0}^{2}(1 / r) \\
& K_{1}=l_{0} /(20 i)-0,75 \leq 1
\end{aligned}
$$

where $l_{0} / i$ ( $i$ being the radius of gyration) denotes the slenderness ratio. The curvature $1 / r$ is given in Eurocode 2 (ENV 1992-1 (2004) as

$$
\begin{aligned}
& 1 / r=2 K_{2} \varepsilon_{\mathrm{yd}} /\left(0,9\left(h-d_{1}\right)\right) \\
& K_{2}=\left(N_{\text {ud }}-N_{\mathrm{d}}\right) /\left(N_{\text {ud }}-N_{\text {bal,d }}\right) \leq 1
\end{aligned}
$$

where $N_{\text {ud }}$ is the design capacity of the cross-section, $N_{\mathrm{d}}$ is the design axial force and $N_{\text {bal,d }}$ is the force which maximises the ultimate moment of the cross-section; in this study (for symmetrical reinforcement) $N_{\text {bal,d }}$ is taken as $N_{\text {bal,d }}=0,5 \alpha f_{\text {cd }} A_{\text {c }}$, where $\alpha$ is a coefficient taking account of long-term effects on the compressive strength. The remaining variables entering equation (7.10), the design yield strain $\varepsilon_{\mathrm{yd}}=f_{\mathrm{yd}} / E_{\mathrm{a}}$ and the effective depth of crosssection $\left(h-d_{1}\right)$, are given below. The resulting values of $N_{\mathrm{d} \text {,max }}$ are shown in Table 7.2.

For given design values of the normal forces $N_{\mathrm{d}}$ and bending moments $M_{\mathrm{d}}$, the column cross-sections are designed using a simplified interaction diagram described by the following formulae:

$$
\begin{aligned}
\text { for } N_{\mathrm{d}}< & \alpha b h f_{\mathrm{cd}} / 2 \\
& {\left[A_{\mathrm{s}} f_{\mathrm{yd}}\left(h-d_{1}-d_{2}\right)+h N_{\mathrm{d}}\left(1-N_{\mathrm{d}} /\left(\alpha b h f_{\mathrm{cd}}\right)\right] / 2-M_{\mathrm{d}}>0\right.} \\
\text { for } N_{\mathrm{d}}> & \alpha b h f_{\mathrm{cd}} / 2 \\
& K_{2}\left[A_{\mathrm{s}} f_{\mathrm{yd}}\left(h-d_{1}-d_{2}\right) / 2+\alpha b h^{2} f_{\mathrm{cd}} / 8\right]-M_{\mathrm{d}}>0 \\
& N_{\mathrm{ud}}=\alpha b h f_{\mathrm{cd}}+A_{\mathrm{s}} f_{\mathrm{yd}}
\end{aligned}
$$




$$
N_{\text {bal, }}=\alpha b h f_{\text {cd }} / 2
$$

These relationships give a good approximation of interaction diagrams derived from appropriate rules. The total reinforcement area $A_{s}$ should satisfy the conditions of Eurocode 2 (ENV 1992-1 (2004)).

$$
\begin{aligned}
& 0,15\left|N_{d}\right| / f_{\mathrm{yd}}<A_{\mathrm{s}}, \\
& 0,003 b h<A_{\mathrm{s}}<0,08 b h
\end{aligned}
$$

This is satisfied in all cases. Theoretical values of $A_{\mathrm{S}}$ rounded off upward to the last digit are given in Table 7.2.

Table 7.2. Effects of actions for the maximum axial force $N_{\mathrm{d} \text {,max }}$.

\begin{tabular}{|c|cccc|c|c|c|c|}
\hline Case & $\begin{array}{c}N_{\mathrm{d}, \max } \\
{[\mathrm{MN}]}\end{array}$ & $\begin{array}{c}M_{\mathrm{d} 0} \\
{[\mathrm{MNm}]}\end{array}$ & $\begin{array}{c}e_{0} \\
{[\mathrm{~m}]}\end{array}$ & $\begin{array}{c}e_{\mathrm{a}} 10^{2} \\
{[\mathrm{~m}]}\end{array}$ & $\begin{array}{c}A_{\mathrm{s}} 10^{4} \\
{\left[\mathrm{~m}^{2}\right]}\end{array}$ & $\begin{array}{c}e_{2} 10^{2} \\
{[\mathrm{~m}]}\end{array}$ & $\begin{array}{c}M_{\mathrm{d}} \\
{[\mathrm{MNm}]}\end{array}$ \\
\hline 1 & 2,075 & 0,329 & 0,159 & 1,7 & 24,9 & 2,2 & 0,410 \\
2 & 2,038 & 0,151 & 0,074 & 0,8 & 19,9 & 0,1 & 0,170 \\
3 & 2,111 & 0,535 & 0,253 & 2,5 & 50,2 & 6,0 & 0,715 \\
4 & 2,148 & 0,768 & 0,358 & 3,4 & 31,4 & 0,3 & 1,061 \\
5 & 1,857 & 0,329 & 0,177 & 1,7 & 40,2 & 2,8 & 0,412 \\
6 & 2,658 & 0,329 & 0,124 & 1,7 & 37,2 & 1,8 & 0,421 \\
7 & 1,660 & 0,263 & 0,159 & 1,7 & 29,2 & 2,9 & 0,339 \\
8 & 2,904 & 0,461 & 0,159 & 1,7 & 31,2 & 1,4 & 0,549 \\
9 & 0,306 & 0,082 & 0,268 & 1,7 & 4,9 & 4,9 & 0,102 \\
10 & 0,671 & 0,137 & 0,204 & 1,7 & 10,7 & 4,9 & 0,181 \\
11 & 4,735 & 0,603 & 0,127 & 1,7 & 55,6 & 0,7 & 0,716 \\
12 & 2,075 & 0,329 & 0,159 & 1,7 & 33,0 & 1,5 & 0,395 \\
\hline
\end{tabular}
sections:

The following assumptions are accepted in the deterministic design of column cross-

- only symmetrical reinforcement $\left(A_{\mathrm{s} 1}=A_{\mathrm{s} 2}=A_{\mathrm{s}} / 2\right)$ is considered;

- the rectangular shape of the column cross-section is chosen such that $h / b=2$ (in the last study case $h / b=3$ );

- the distance of reinforcing bars from the edge is chosen in all study cases as $d_{1}=0,05 \mathrm{~m}$; and

- the assumed material characteristics for the concrete class C 20/25 and reinforcing steel S 500 are

$$
\begin{aligned}
& f_{\mathrm{ck}}=20 \mathrm{MPa}, \gamma_{\mathrm{c}}=1,5, f_{\mathrm{cd}}=13,33 \mathrm{MPa}, \alpha=0,85 \\
& f_{\mathrm{yk}}=500 \mathrm{MPa}, \gamma_{\mathrm{s}}=1,15, f_{\mathrm{yd}}=435 \mathrm{MPa}
\end{aligned}
$$

\subsection{Limit state function}

In the time-variant reliability analysis the actual axial force $N$ is considered as a simple sum of actual axial forces due to all considered actions:

$$
N=N_{\mathrm{W}}+N_{\text {imp }}+N_{\text {wind }}
$$


where $N_{\mathrm{W}}$ is the axial force due to self-weight, $N_{\text {imp }}$ is the axial force due to imposed load and $N_{\text {wind }}$ is the axial force due to wind action (positive values are again accepted for compressive forces). The bending moment $M$ is given by a modified equation (7.6), in which actual values are applied instead of the design values and a new additional eccentricity $e_{\mathrm{a}}$ is considered as $\zeta$ $L / 2$, where the initial sway $\zeta$, is given in Table 7.3. The second order eccentricity $e_{2}$ is given by modified equations (7.8) and (7.9), in which $l_{0}=L$.

The limit state function $G(\boldsymbol{X})$ may be expressed as the difference between the resistance bending moment and the actual bending moment about the cross-section centre

$$
G(X)=\xi_{\mathrm{R}} M_{\mathrm{R}}-\xi_{\mathrm{E}} M
$$

Two coefficients of model uncertainties $\xi_{\mathrm{R}}$ and $\xi_{\mathrm{E}}$ are considered as random variables to cover the imprecision and incompleteness of the relevant theoretical models. The resistance bending moment is determined taking into account (7.15) to (7.18), where actual values of basic variables are considered instead of the design values. The statistical characteristics of all basic variables, based on the data given in a previous study by Holický \& Vrouwenvelder [31], and JCSS document [27] are shown in Table 7.3.

Table 7.3. Statistical characteristics of basic variables.

\begin{tabular}{|l|l|c|c|c|c|}
\hline Symbol & Name of variable & $\begin{array}{c}\text { Distrib. } \\
\text { /Type }\end{array}$ & $\begin{array}{c}\text { Dimen- } \\
\text { sion }\end{array}$ & Mean & $\begin{array}{c}\text { Standard } \\
\text { deviation }\end{array}$ \\
\hline$\alpha$ & reduction factor & $\mathrm{R} / \mathrm{R}$ & - & 0,85 & 0,085 \\
$A_{\mathrm{s}}$ & reinforcement area & $\mathrm{DET}$ & $\mathrm{m}$ & nom & 0 \\
$f_{\mathrm{c}}$ & concrete strength & $\mathrm{LN} / \mathrm{R}$ & $\mathrm{MPa}$ & 30 & 5 \\
$f_{\mathrm{y}}$ & yield strength & $\mathrm{LN} / \mathrm{R}$ & $\mathrm{MPa}$ & 560 & 30 \\
$E$ & modulus of elast. & $\mathrm{DET}$ & $\mathrm{GPa}$ & 200 & 0 \\
\hline$a_{1}$ & column distance & $\mathrm{DET}$ & $\mathrm{m}$ & nom & 0 \\
$a_{2}$ & perpend. distance & $\mathrm{DET}$ & $\mathrm{m}$ & nom & 0 \\
$b$ & width of section & $\mathrm{N} / \mathrm{R}$ & $\mathrm{m}$ & nom & 0,005 \\
$d_{1}, d_{2}$ & distance of bars & $\mathrm{N} / \mathrm{R}$ & $\mathrm{m}$ & $0,05 \mathrm{~m}$ & 0,01 \\
$h$ & height of section & $\mathrm{N} / \mathrm{R}$ & $\mathrm{m}$ & nom & 0,01 \\
$L$ & height of column & $\mathrm{DET}$ & $\mathrm{m}$ & nom & 0 \\
$n$ & number of floors & $\mathrm{DET}$ & - & nom & 0 \\
$\zeta$ & initial sway & $\mathrm{N} / \mathrm{R}$ & $\mathrm{rad}$ & 0 & $0,001^{(1)}$ \\
\hline$\xi_{E}$ & mod. unc. load & $\mathrm{N} / \mathrm{R}$ & - & 1,0 & 0,1 \\
$\xi_{R}$ & mod. unc. resistance & $\mathrm{N} / \mathrm{R}$ & - & 1,1 & 0,11 \\
\hline$\rho$ & weight & $\mathrm{N} / \mathrm{R}$ & $\mathrm{MNm}^{-2}$ & 0,0240 & 0,00192 \\
$C_{\mathrm{p}}$ & shape factor & $\mathrm{N} / \mathrm{R}$ & - & 1,3 & 0,13 \\
$G$ & gust factor & $\mathrm{GUM} / \mathrm{R}$ & - & 2,5 & 0,25 \\
$p_{\text {wind }}$ & wind pressure & $\mathrm{GUM} / \mathrm{S}$ & $\mathrm{MNm}^{-2}$ & 0,00035 & $0,00006^{(2)}$ \\
$p_{\text {impl }}$ & long-term load & $\mathrm{GAM} / \mathrm{S}$ & $\mathrm{MNm}^{-2}$ & 0,0006 & mean $\times v^{(3)}$ \\
$p_{\text {imps }}$ & short-term load & $\mathrm{GAM} / \mathrm{S}$ & $\mathrm{MNm}^{-2}$ & 0,0002 & mean $\times v^{(4)}$ \\
\hline
\end{tabular}

Notes to Table 7.3:

(1) The initial overall sway $\zeta$ is used to calculate the additional eccentricity $e_{\mathrm{a}}$ according to equation $e_{\mathrm{a}}=\zeta L / 2$.

(2) The mean and standard deviation correspond to the distribution of the yearly maximum.

(3) The mean and standard deviation correspond to the random point-in-time distribution; $v^{2}=\left(0,16+8 /\left(a_{1} a_{2}\right)\right)\left(1 / n+\rho_{\text {impl }}(1-1 / n)\right)($ CIB report, see [32]), where the coefficient of the correlation of the long-term loads in two floors is considered as $\rho_{\text {impl }}=0,5$. The factor $v$ also holds for one storey with $\mathrm{n}=1$.

(4) The mean and standard deviation correspond to the distribution of 24 hours (one day) maximum, $v^{2}=50 /\left(a_{1} a_{2}\right)$. 
In Table 7.3 all time-invariant variables are denoted by R-variables while time-variant variables are denoted by $\mathrm{S}-\mathrm{V}$ ariables for later convenience.

\subsection{Reliability analysis}

The reliability analysis of the column is presented for four different assumptions considering variable actions as:

- time-invariant variable models for extreme wind, long- and short-term imposed loads - the resulting reliability indices $\beta_{0}$ may be used as a first approximation of a more refined analysis,

- jump processes without intermittencies for wind and long-term imposed loads - the resulting reliability indices $\beta_{1}$ refer to Turkstra's rule (see ISO 2394 [2]), i.e. a load takes on its extreme while the others are at their point-in-time value and the resulting probabilities are summed,

- jump processes without intermittencies for wind, long- and short-term imposed loads - the resulting reliability indices are denoted by $\beta_{2}$,

- jump processes with intermittencies for wind, for simultaneously jumping long-term loads and for short-term loads - the resulting reliability indices are denoted by $\beta_{3}$.

- jump processes with intermittencies with a given interarrival duration intensity for wind, long- and short-term loads - the resulting reliability indices $\beta_{4}$ refer to independent occupancy loads.

In the time-invariant analysis all the variable actions are considered as time-invariant processes characterised by the theoretical models indicated in Table 7.3. The time-variant reliability analysis assumes that the variable actions (wind, short-term and long-term imposed loads) are stationary and ergodic random processes described by rectangular wave renewal processes (jump processes) enveloping the real load processes. In this sense all subsequent results are conservative. When no intermittencies are considered, then each jump process is characterised by a jump rate $\lambda$ (an average number of the magnitude changes of square waves in a year). Two different analyses assuming the jump processes without intermittencies, described in detail by Holický \& Vrouwenvelder [31], are presented here: short-term imposed load is absent $\left(\beta_{1}\right)$ and short-term load is present $\left(\beta_{2}\right)$.

When intermittencies are considered, in addition to the jump rate $\lambda$, each jump process is additionally characterised by the interarrival duration intensity $\rho$ (a product of the arrival rate and the mean duration $\mu_{\eta}$ ), both expressed in terms of years. The distribution of the duration of a rectangular wave can theoretically remain unspecified. Its mean duration is $1 / \lambda$. However, the distributions for the times between the interarrivals of on-times and the distributions of the durations of on-times must be specified.

For stationary processes and stationary intermittencies and assuming random initial conditions an upper-bound failure probability is determined from

$$
\begin{aligned}
& P_{f}\left(t_{1}, t_{2}\right) \leq P_{f}\left(t_{1}\right)+E\left[N^{+}\left(t_{1}, t_{2}\right)\right] \\
& P_{f}\left(t_{1}\right)=\sum_{i=1}^{n} p_{1}^{i} P_{f, 1}^{i}\left(t_{1}\right)+\sum_{i=1}^{n} \sum_{j=i+1}^{n} p_{2}^{i, j} P_{f, 2}^{i, j}\left(t_{1}\right)+\ldots+p_{n}^{1,2, \ldots, n} P_{f, n}^{1,2, ., n}\left(t_{1}\right) \\
& E\left[N^{+}\left(t_{1}, t_{2}\right)\right]=\sum_{i=1}^{n} v_{i}^{+}\left\{p_{1}^{i}\left(t_{2}-t_{1}\right)\right\}+\sum_{i=1}^{n} \sum_{j=i+1}^{n} v_{i+j}^{+}\left\{p_{2}^{i, j}\left(t_{2}-t_{1}\right)\right\}+ \\
& \ldots+v_{1+2+\ldots+n}^{+}\left\{p_{n}^{1,2, . ., n}\left(t_{2}-t_{1}\right)\right\}
\end{aligned}
$$


where $\left[t_{1}, t_{2}\right]$ denotes the reference time interval, $E\left[N^{+}\left(t_{1}, t_{2}\right)\right]$ the mean number of outcrossings and $v^{+}\left(t_{1}, t_{2}\right)$ the outcrossing rate. The $p_{k}^{\{k\}}$ are the coincidence probabilities for the set $\{k\}$ of loads being "on". They are computed according to a simple model proposed earlier (see [32]). There it is assumed that the interarrival times of the "on"-times follow an exponential distribution with a parameter $\kappa$ and the duration of "on"-times a truncated exponential distribution. Each "on"-time is associated with only one rectangular wave so that with $\lambda=\kappa=1 / \mu_{\tau}$ the interarrival intensity is $\rho=\mu_{\eta} / \mu_{\tau}=\lambda \mu_{\eta}$ (see Figure 7.2).

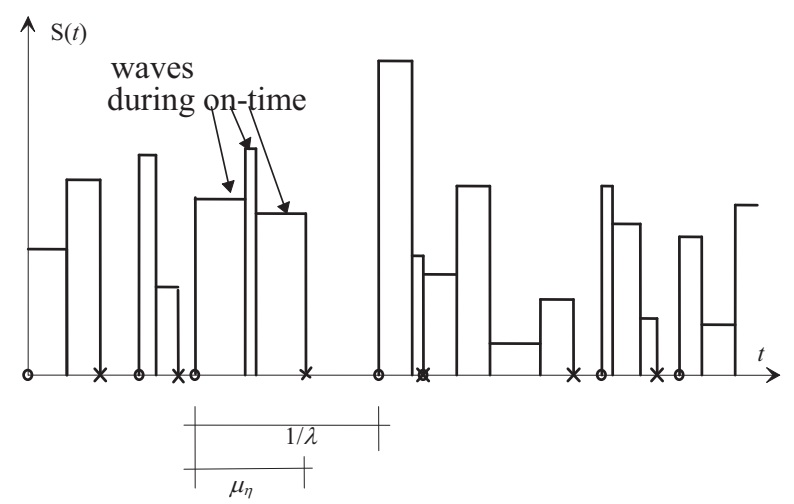

Figure 7.2. Intermittent rectangular wave jump process.

The outcrossing rate for the independent amplitudes of the rectangular wave renewal processes is determined from (Rackwitz [40])

$$
\begin{aligned}
& v_{\{k\}}^{+}\left(F_{\{k\}}, \tau\right) \approx \sum_{i=1}^{n_{s}} \lambda_{i}\left[\frac{\Phi\left(-\beta_{\{k\}}\right)}{\prod_{j=1}^{n_{s}-1}\left(1-\beta_{\{k\}} \kappa_{j}\right)^{1 / 2}}-\Phi\left(-\beta_{\{k\}},-\beta_{\{k\}} ; \rho_{\{k\}_{i}}\right)\right]=\frac{\Phi\left(-\beta_{\{k\}}\right)}{\prod_{j=1}^{n_{s}-1}\left(1-\beta_{\{k\}} \kappa_{j}\right)^{1 / 2}} \\
& * \sum_{i=1}^{n_{s}} \lambda_{i}\left[1-\frac{\Phi\left(-\beta_{\{k\}},-\beta_{\{k\}} ; \rho_{\{k\}_{j}}\right)}{\Phi\left(-\beta_{\{k\}}\right) \prod_{j=1}^{n_{s}-1}\left(1-\beta_{\{k\}} \mathcal{K}_{\{k\}_{j}}\right)^{-1 / 2}}\right]=\Phi\left(-\beta_{\{k\}}\right) \prod_{j=1}^{n_{s}-1}\left(1-\beta_{\{k\}} \kappa_{\{k\}_{j}}\right)^{-1 / 2} \sum_{i=1}^{n_{s}} \lambda_{\{k\}}^{\prime} i
\end{aligned}
$$

$\beta_{\{k\}}$ is the reliability index and $\kappa_{\{k, j}$ are the main curvatures in the $\beta$-point according to FORM/SORM for the set $\{\mathrm{k}\}$ of active loads. $\rho_{\{k\}}$ is the correlation coefficient of the two linearised failure domains before and after a jump. As usual, the reliability computations are performed in a standard space and the results given below correspond to SORM.

Formally, the last factor can be interpreted as a first-order correction to the jump rates, then denoted by $\lambda^{\prime}$. It is an improvement as compared to the asymptotic result [31]. The improvement is demonstrated in Figure 7.3. Amplitude dependencies as in Table 7.3 can also be introduced by making the mean of the imposed long-term load uncertain, for example, as a normally distributed variable with the mean zero. Then $\rho_{\text {impl }}=0,5$ corresponds to a standard deviation of this uncertain mean of about $70 \%$ of the original standard deviation and the standard deviation of $p_{\text {impl }}$ given the mean is also only about $70 \%$ of the original value of $\left(0,16+8 /\left(a_{1} a_{2}\right)\right)$. 


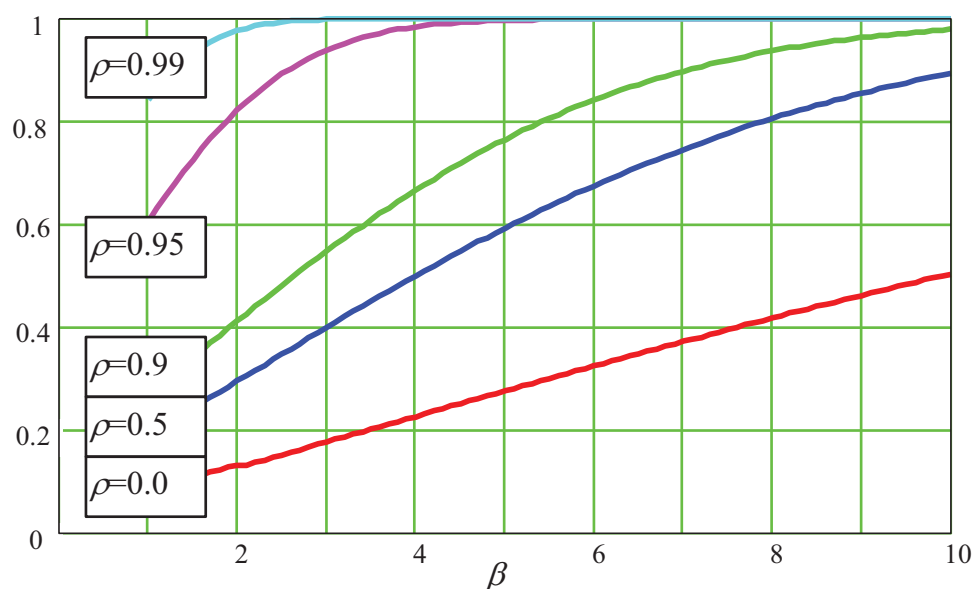

Figure 7.3. Ratio of improved and asymptotic domain probabilities over reliability index $\beta$ for various $\rho$.

A lower bound for the failure probability can also be given as the point-in-time failure probability

$$
\begin{aligned}
& P_{f}\left(t_{1}, t_{2}\right) \geq \max _{\tau}\left\{\sum_{i=1}^{n} p_{1}^{i} P_{f, 1}^{i}(\tau)+\sum_{i=1}^{n} \sum_{j=i+1}^{n} p_{2}^{i, j} P_{f, 2}^{i, j}(\tau)+\ldots+p_{n} P_{f, n}^{1,2, \ldots, n}(\tau)\right\} \\
& \geq \sum_{i=1}^{n} p_{1}^{i} P_{f, 1}^{i}\left(t_{i}^{*}\right)+\sum_{i=1}^{n} \sum_{j=i+1}^{n} p_{2}^{i, j} P_{f, 2}^{i, j}\left(t_{i, j}^{*}\right)+\ldots+p_{n} P_{f, n}^{1,2, \ldots, n}\left(t_{1,2, \ldots, n}^{*}\right)
\end{aligned}
$$

where, due to stationarity, $t_{\{k\}}^{*}$ can be taken as $t_{l}$.

The following parameters are considered here:

- long-term imposed load:

$\lambda=\kappa=1 / 7$ [1/ year], $\rho=3$ (an assumption),

- short-term imposed load:

$\lambda=\kappa=n[1 /$ year $], \rho=n / 365$,

- wind load:

$\lambda=\kappa=10[1 /$ year $], \rho=10 / 365 / 3=0,009$,

where $n$ denotes the number of storeys (equal to 1, 3 and 10).

Thus, the long-term imposed load is assumed to change simultaneously in all storeys of a building on average every 7 years. The assumption of $\rho=3$ may be a little unrealistic but the influence of the parameter $\rho$ of the long-term imposed load is not very important.

The short-term load is assumed to be present in each storey of the building during one day in a year. Approximately (neglecting possible dependence and time overlapping of the load in different storeys), the short-term load is considered as one process with a number of magnitude changes in a year $\lambda=n$ and the interarrival duration intensity $\rho=\lambda \mu_{\eta}=n / 365$ 
corresponding to the arrival rate and to the mean duration $\mu_{\eta}$ of one day. This may not be an adequate approximation in some cases as it implies that short-term loadings occur independently and do not overlap in time.

Further, the extreme wind velocity in a year is assumed to have the characteristics specified in Table 7.3, and the intensity $\rho$ for wind is determined assuming an arrival rate of on-times 10 (ten storms in a year) with the mean duration $\mu_{\eta}$ of 8 hours (1/365/3 of a year). In a first approximation the pressure distribution in each storm is again Gumbel with the same standard deviation but with an appropriately reduced mean.

The resulting reliability indices $\beta_{0}, \beta_{1}, \beta_{2}, \beta_{3}$ and $\beta_{4}$ for the 12 study cases are given in Table 7.4, assuming a lifetime of 50 years.

Table 7.4. Reliability indices for a built-in column.

\begin{tabular}{|c|c|ccccc|}
\hline Study & Reinf. & \multicolumn{5}{|c|}{ Reliability analysis } \\
\cline { 3 - 7 } & $\begin{array}{c}\text { ratio } \\
{[\%]}\end{array}$ & Time invar. & $\begin{array}{c}\text { Turkstra's } \\
\text { rule }\end{array}$ & $\begin{array}{c}\text { Jump } \\
\text { process }\end{array}$ & Intermittent & Intermittent \\
& $A_{S} / b h$ & $\beta_{0}$ & $\beta_{1}$ & $\beta_{2}$ & $\beta_{3}$ & $\beta_{4}$ \\
\hline 1 & 1,02 & 5,34 & 5,31 & 5,30 & 5,98 & - \\
2 & 1,11 & 5,95 & 6,03 & 6,03 & 6,69 & - \\
3 & 2,05 & 5,07 & 5,05 & 5,04 & 5,76 & - \\
4 & 0,78 & 4,06 & 3,99 & 3,97 & 4,83 & - \\
5 & 2,23 & 5,27 & 5,34 & 5,33 & 6,08 & - \\
6 & 1,52 & 6,08 & 6,07 & 6,07 & 6,69 & - \\
7 & 1,62 & 5,38 & 5,35 & 5,35 & 6,04 & - \\
8 & 0,98 & 5,57 & 5,55 & 5,54 & 6,21 & - \\
9 & 0,39 & 2,85 & 2,70 & 2,60 & 3,75 & 3,75 \\
10 & 0,86 & 3,69 & 3,59 & 3,55 & 4,48 & 4,29 \\
11 & 1,37 & $5,59^{*}$ & $5,69^{*}$ & $5,64^{*}$ & $6,32^{*}$ & - \\
12 & 1,76 & $5,62^{*}$ & $5,68^{*}$ & 5,66 & 6,35 & - \\
\hline
\end{tabular}

In all the cases rapid convergence to a unique $\beta$-point was observed. The given results correspond to SORM. A few results have been checked by crude Monte Carlo integration or by importance sampling showing errors of, at most, a few per cent. The numerical effort for the five types of analysis was about 1:3:1:6:7 with the unit being approximately 500 state function calls. The reliability indices are within a wide range from less than 3 to over 7 (similarly as in previous studies [31]). When judging the results, one has to know whether long- and short-term occupancy loadings are "loading" or "resistance" variables. These loads can have a stabilising effect. Whenever one of them is a "loading" variable, this is indicated by the superscript " ",".

The time-invariant calculations for $\beta_{0}$ and $\beta_{1}$ are approximate in the sense that the extreme value distributions described by gamma-distributions are replaced by Gumbel distributions with the same mean and variance. $\beta_{0}$ is in general far too inaccurate and should be considered as a first approximation only. In all the study cases $\beta_{0}, \beta_{1}$, and $\beta_{2}$ (no intermittencies) differ insignificantly and all are always smaller than $\beta_{3}$ (intermittencies). This is due to dominating wind load. However, $\beta_{2}$ and $\beta_{3}$ can generally be markedly different from $\beta_{0}$ and $\beta_{1}$. Note that in the study cases 9 and 10 the reliability indices $\beta_{0}, \beta_{1}$ and $\beta_{2}$ (in the study case 9 also $\beta_{3}$ and $\beta_{4}$ ) are less than 3,8, which is the value recommended in ENV1991-1: Eurocode 1 (1994).

Figure 7.4 shows the reliability indices $\beta_{2}$ and $\beta_{3}$ versus the reinforcement ratio. It appears that for a reinforcement ratio less than $1 \%$, both the reliability indices (and as follows from Table 7.4 also $\beta_{0}, \beta_{1}$ and $\beta_{2}$ ) considerably decrease and may be unsatisfactory. 


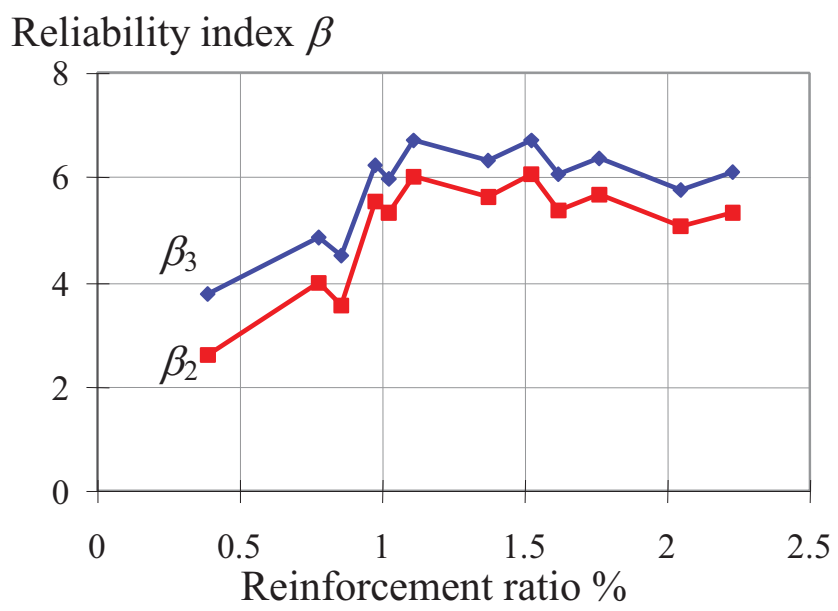

Figure 7.4. Reliability indices $\beta_{2}<\beta_{3}$ versus the reinforcement ratio.

\subsection{Study case $\mathbf{1 0}$}

The assumption that the long-term imposed load changes simultaneously in all storeys of a building (on average every 7 years) may not be realistic. In the following this hypothesis is changed to another extreme assumption. The long-term imposed loads in particular storeys are mutually independent. So, in the study case 10, having three storeys, three independent long-term imposed loads are considered together with a short-term imposed load and wind load. Thus, in total $2^{\mathrm{n}}-1=31$ different "load combination cases" have to be studied, i.e. any one load "on" and all others "off", any two loads "on" and the others "off", ..., all 5 different loads "on". The probability of failure for all loads being "off" is neglected. For the fourth type of analysis there are only 7 "load combinations". For the convenience of presentation only these combinations are shown in the Table 7.5. It is seen that larger contributions to the total failure probability come only when wind is active. In this case occupancy loading has a stabilising effect. The computations with simultaneous jumps $\left(\beta_{3}\right)$ result in larger $\beta$-values than with independent jumps. The detailed analysis shows that this is due to a larger total jump rate but also due to the correlation between the loads for simultaneous jumps.

The upper and lower (approximate) bounds of the failure probability $P_{f}$, defined by (7.22) and (7.24), are indicated in Figure 7.5 in a parameter study concerning the interarrival duration intensity $\rho$ of wind load within a range from 0,005 to 0,015 (the value assumed in the previous calculation was 0,009$)$ implying a change in the duration of the storms from roughly $50 \%$ (4 hours) to $150 \%$ (12 hours).

Figure 7.5 shows that the upper and lower bounds are very close to each other. For example, for $\rho=0.009$ (used in the previous analysis) it holds

$$
4,48<\beta<4,63
$$




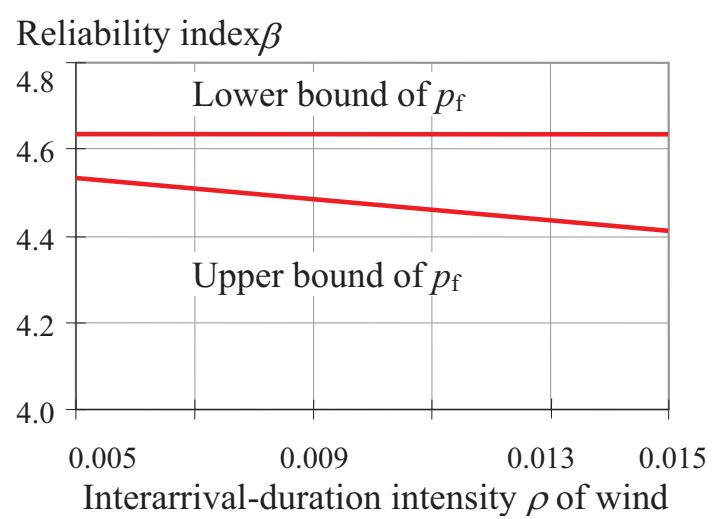

Figure 7.5. Lower and upper bounds of failure probability $p_{\mathrm{f}}$ for the study case $10\left(\beta_{3}\right)$.

It can be shown that the true reliability is very close to the upper bound in this case. The lower bound also corresponds to the initial failure probability.

Table 7.5. Load combinations for the study case 10 for $\beta_{3}$ (sequence is $p_{\text {wind }}, p_{\text {imps }}, p_{\text {impl }}$ ).

\begin{tabular}{|c|c|c|c|}
\hline Case & Active load & $p_{\mathrm{f}}\{\mathrm{k}\}$ & $p\{\mathrm{k}\}$ \\
\hline 1 & xxx & $3,93 \mathrm{E}-07$ & $5,45 \mathrm{E}-05$ \\
\hline 2 & xoo & $1,09 \mathrm{E}-05$ & $2,21 \mathrm{E}-03$ \\
\hline 3 & oxo & $2,44 \mathrm{E}-23$ & $2,02 \mathrm{E}-03$ \\
\hline 4 & oox & $3,27 \mathrm{E}-23$ & $7,37 \mathrm{E}-01$ \\
\hline 5 & xxo & $5,04 \mathrm{E}-07$ & $1,82 \mathrm{E}-05$ \\
\hline 6 & xox & $1,70 \mathrm{E}-06$ & $6,64 \mathrm{E}-03$ \\
\hline 7 & oxx & $2,72 \mathrm{E}-23$ & $6,06 \mathrm{E}-03$ \\
\hline
\end{tabular}

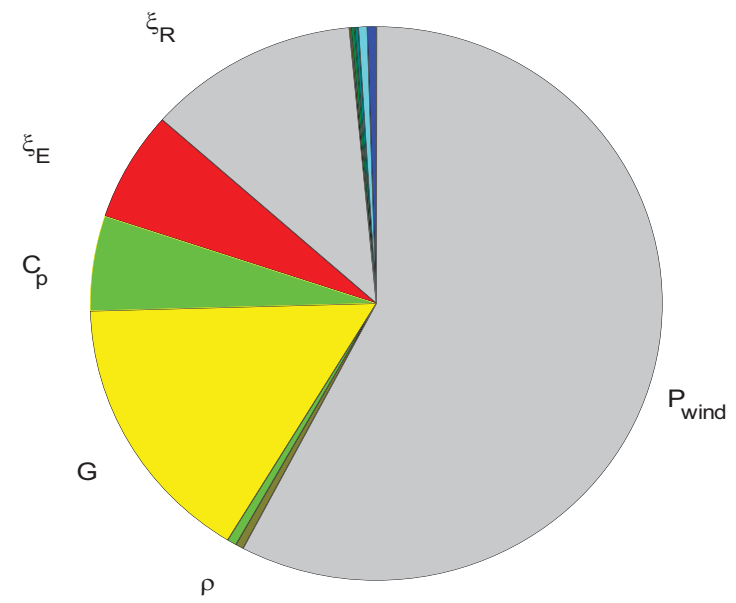

Sum of $a^{\wedge} 2=1.0$

Figure 7.6. $\alpha$-values of variables $\left(\alpha_{\mathrm{PL} 1}=\alpha_{\mathrm{PL} 2}=\alpha_{\mathrm{PL} 3}=0,0109\right)$. 
Finally, it follows from Figure 7.6 that wind is the dominating variable and live loads are "resistance" variables. The corresponding $\alpha$-values are positive but small.

\subsection{Concluding remarks}

The results of the reliability analysis of 12 study cases of a reinforced concrete column show considerable differences in the reliability level of the column in different structural arrangements. Considering a 50-year lifetime, reliability indices vary within a broad range of from 2,6 up to 6,7 .

Any time-invariant analysis is inadequate. The time-variant reliability analysis assuming variable loads as jump processes without intermittencies is a safe approximation to a more refined analysis assuming variable loads as jump processes with intermittencies. The latter type of analysis may considerably improve the reliability assessment of structures, particularly those exposed to variable actions with relatively low interarrival duration intensity. It is further emphasised that the effect of stabilising occupancy loading can only be taken into account consistently by assuming jump processes with intermittencies.

It appears that the reliability level of reinforced concrete columns designed according to Eurocodes may in some cases be insufficient. In other cases, depending on actual structural arrangements, it may become uneconomical to follow the codes. The obtained reliability indices $\beta$ seem to be dependent on the reinforcement ratio. In particular, lower $\beta$-values were obtained for reinforcement ratios below $1 \%$.

The widely varying reliability indices suggest that the partial safety factors in the Eurocodes are not necessarily appropriate. It appears that larger partial safety factors for variable loading, smaller safety factors for dead load and resistance variables, especially a better load combination rule, would make the reliability level more uniform. Definite conclusions, however, must be based on a much larger set of study cases.

The detailed analysis of the study case 10 shows that lower and upper bounds on failure probabilities are very near to each other. The independence of jumps of long-term imposed loads in different storeys leads to a decrease of $\beta$ by 0,15 (increase of failure probability). Obviously, the assumptions concerning the dependence of long-term imposed loads should be revised in other study cases also. 


\subsection{Introduction}

Partial factor methods provide operational design procedures adopted in many national and international standards, including Eurocodes. The fundamental Eurocode EN 1990 Basis of Structural Design [1] describes details of accepted procedures based on a partial factor method in Section 6 and Annex A. Additional background information and the theoretical basis of the method are described in Annex C of EN 1990 [1]. General concepts of the partial factor method are also described in International Standard ISO 2394 [2].

This chapter is primarily focused on the design verification formats of partial factors adopted in the latest version of Eurocodes. Essentially, it is a short extension of chapters 5 and 6 , which describe how to determine partial factors for material properties and actions using general probabilistic concepts.

Symbols and terms used in EN 1990 [1] coincide mostly with those applied in this chapter. However, EN 1990 does not use capitals and small letters in the same way as introduced in previous text (where capitals denote general random variables and small letters their particular realisations). In Eurocodes a particular value of a random variable is denoted by a subscript of the letter denoting the variable. For example, in EN 1990 a material property as a random variable is denoted by $X$, its particular value, say the design value, is denoted by $X_{\mathrm{d}}$ (while $x_{\mathrm{d}}$ would be used in previous chapters). As this chapter is closely linked to Eurocodes, notation used in these documents is accepted here (i.e. the symbol $X$ denotes a material property and $X_{\mathrm{d}}$ is used for its design value).

It should also be noted that alternative terms and symbols can be found in ISO 2394 and other literature on structural reliability. For example the term "performance function" used in Annex C of EN 1990 is sometimes replaced by "limit state function" or "state function". Another important term "survival probability" $p_{\mathrm{s}}$ is often called "reliability" (see also the recent document ISO 2394 [2]). Other terms used in Annex C of EN 1990, for example "probabilistic, semi-probabilistic, deterministic methods" and the classification of "level I, II and III reliability methods", may be applied in literature in a slightly different way. Additional terms (like "design value method" used previously in ENV 1991-1 but not applied in EN 1990) may be found in ISO 2394 [2] and other literature.

Supplementary symbols and terms to those given in EN 1990 will be introduced in this chapter in order to provide more comprehensive background information. For example $\varphi(x)$ will be used for the probability density function of a variable $X$, symbol $X$ will be also used for a vector of all basic variables.

\subsection{The design value method}

The design value method, which is also called "semi-probabilistic method (level I)" (in EN 1990 [1]), is a very important step from probabilistic design methods toward operational partial factors method. The design value method is directly linked to the basic principle of EN 1990, according to which it should be verified that no limit state is exceeded when the design values of all basic variables are used in the models of structural resistance $R$ and action effect $E$. Thus, if the design values $E_{\mathrm{d}}$ and $R_{\mathrm{d}}$ of $E$ and $R$ are determined considering the design values of all basic variables, then a structure is considered as reliable when the following expression holds

$$
E_{\mathrm{d}}<R_{\mathrm{d}}
$$


where the design values $E_{\mathrm{d}}$ and $R_{\mathrm{d}}$ are symbolically expressed as

$$
\begin{gathered}
E_{\mathrm{d}}=E\left\{F_{\mathrm{d} 1}, F_{\mathrm{d} 2}, \ldots a_{\mathrm{d} 1}, a_{\mathrm{d} 2}, \ldots \theta_{\mathrm{d} 1}, \theta_{\mathrm{d} 2}, \ldots\right\} \\
R_{\mathrm{d}}=R\left\{X_{\mathrm{d} 1}, X_{\mathrm{d} 2}, \ldots a_{\mathrm{d} 1}, a_{\mathrm{d} 2}, \ldots \theta_{\mathrm{d} 1}, \theta_{\mathrm{d} 2}, \ldots\right\}
\end{gathered}
$$

Here, $E$ denotes a function describing the action effect, $R$ denotes a function describing the structural resistance, $F$ is a general symbol for actions, $X$ for material properties, $a$ for geometrical properties, and $\theta$ for model uncertainties. Subscript 'd' refers to design values.

If only two variables $E$ and $R$ are considered, then the design values $E_{\mathrm{d}}$ and $R_{\mathrm{d}}$ may be found using the following formulae

$$
\begin{gathered}
\mathrm{P}\left(E>E_{\mathrm{d}}\right)=\Phi\left(+\alpha_{E} \beta\right) \\
\mathrm{P}\left(R \leq R_{\mathrm{d}}\right)=\Phi\left(-\alpha_{R} \beta\right)
\end{gathered}
$$

where $\beta$ is the target reliability index, $\alpha_{E}$ and $\alpha_{R}$, with $|\alpha| \leq 1$, are the values of the FORM weight (sensitivity) factors. As already indicated in equations (5.26) and (5.28), the sensitivity factor $\alpha_{E}$ is negative for unfavourable actions and action effects (in EN 1990 [1] $\alpha_{E}=-0,7$ ), the resistance sensitivity factor $\alpha_{R}$ is positive (in EN 1990 [1] $\alpha_{R}=0,8$ ).

\subsection{Reliability verification in Eurocodes}

In accordance with the partial factor methods accepted in EN 1990 to 1999 the design values of the basic variables, $X_{\mathrm{d}}$ and $F_{\mathrm{d}}$, are usually not introduced directly into the design equations. They are commonly expressed in terms of their representative values $X_{\text {rep }}$ and $F_{\text {rep}}$, which may be:

- the characteristic values $X_{\mathrm{k}}$ and $F_{\mathrm{k}}$, i.e. values with a prescribed or intended probability of being exceeded, for example for actions, material properties and geometrical properties;

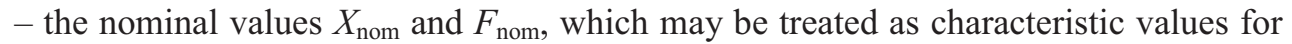
material properties and as design values for geometrical properties.

The representative values $X_{\text {rep }}$ and $F_{\text {rep }}$ should be divided and/or multiplied, respectively, by the appropriate partial factors to obtain the design values $X_{\mathrm{d}}$ and $F_{\mathrm{d}}$. Considering the representative values $X_{\text {rep }}$ and $F_{\text {rep }}$ by their characteristic values $X_{\mathrm{k}}$ and $F_{\mathrm{k}}$, the design values $X_{\mathrm{d}}$ and $F_{\mathrm{d}}$ can be expressed as

$$
\begin{gathered}
X_{\mathrm{d}}=X_{\mathrm{k}} / \gamma_{\mathrm{M}} \\
F_{\mathrm{d}}=\gamma_{F} F_{\mathrm{k}}
\end{gathered}
$$

where $\gamma_{M}$ denotes the partial factor of materials properties and $\gamma_{F}$ the partial factor of action. Both partial factors $\gamma_{M}$ and $\gamma_{F}$ are in most cases greater than 1 . The above expressions for the design values of actions $F$ and material properties $X$ may be modified depending on the type of verified structural member and construction material.

As described in the following sections, both partial factors $\gamma_{M}$ and $\gamma_{F}$ should include model uncertainties, which may significantly affect the reliability of a structure. As stated in EN 1990 [1], design values for model uncertainties may be incorporated into the design expressions through the partial factors $\gamma_{E \mathrm{~d}}$ and $\gamma_{R \mathrm{~d}}$ applied as follows:

$$
E_{d}=\gamma_{E d} E\left\{\gamma_{g j} G_{k j} ; \gamma_{P} P ; \gamma_{q 1} Q_{k 1} ; \gamma_{q i} \psi_{0 i} Q_{k i} ; a_{d} \ldots\right\}
$$




$$
R_{d}=R\left\{\eta X_{k} / \gamma_{m} ; a_{d} \ldots\right\} / \gamma_{R d}
$$

Here $\eta$ denotes a conversion factor appropriate to the material property.

The coefficient $\psi$, which takes account of reductions in the design values of variable actions, is applied as $\psi_{0}, \psi_{1}$ or $\psi_{2}$ to simultaneously occurring accompanying variable actions. The following simplifications may be made to expressions (8.8) and (8.9), when required.

a) On the loading side (for a single action or where linearity of action effects exists) :

$$
E_{\mathrm{d}}=E\left\{\gamma_{\mathrm{F}, \mathrm{i}} F_{\text {rep }, \mathrm{i}}, a_{\mathrm{d}}\right\}
$$

b) On the resistance side the general format is given in expressions (8.9), and further simplifications may be modified in the relevant material-oriented documents provided the level of reliability is not reduced. It should be mentioned that non-linear resistance and action models, and multi-variable action or resistance models, are commonly encountered in Eurocodes. In such instances, the above relations become more complex.

\subsection{Partial factors in Eurocodes} in Figure 8.1.

The relation between individual partial factors in Eurocodes is schematically indicated

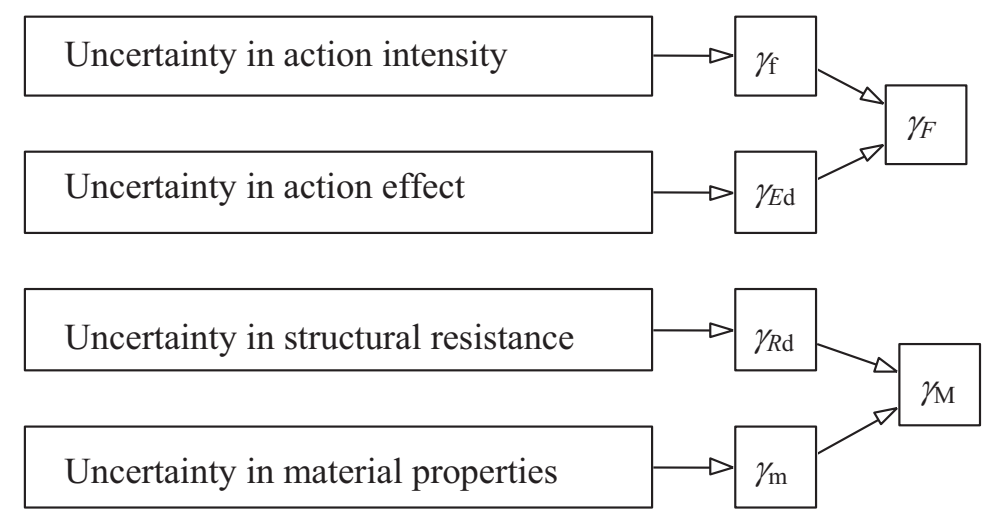

Figure 8.1: Relation between individual partial factors

In accordance with Figure 8.1 the partial factor $\gamma_{F}$ may be split into the load intensity uncertainty factor $\gamma_{\mathrm{f}}$ and model uncertainty factor $\gamma_{E \mathrm{~d}}$. Similarly, the partial factor $\gamma_{\mathrm{M}}$ may be split into the material property factor $\gamma_{\mathrm{m}}$ and resistance model uncertainty factor $\gamma_{R \mathrm{~d}}$. Generally, it holds that

$$
\begin{aligned}
& \gamma_{\mathrm{F}}=\gamma_{\mathrm{f}} \gamma_{E \mathrm{~d}} \\
& \gamma_{\mathrm{M}}=\gamma_{\mathrm{m}} \gamma_{R \mathrm{~d}}
\end{aligned}
$$

Note that in EN 1990 subscript " $S$ " is used for uncertainty of the action effects instead of symbol " $E$ ", which is used consistently here. Numerical values of both factors of model uncertainty depend on particular conditions and should be derived from previous experience and available experimental data. The load effect factor $\gamma_{E \mathrm{~d}}$ may be expected within the interval from 
1,05 to 1,15 (as indicated in EN 1990 [1]). The resistance factor $\gamma_{R \mathrm{~d}}$ depends on the construction materials and behaviour of the structural member. For example, uncertainty of the bending capacity of a steel beam will be lower (about 1,05 ) than uncertainty of a welded connection capacity (about 1,15). Similarly, uncertainty of the bending moment capacity of a reinforced concrete beam will be lower than uncertainty of the shear capacity of the beam.

\subsection{Partial factors for material property}

Partial factor for resistance $\gamma_{\mathrm{m}}$ is defined in equation (8.13) by fractiles $X_{\mathrm{k}}$ and $X_{\mathrm{d}}$. Taking into account general expression (4.2) for fractiles of random variables the factor $\gamma_{\mathrm{m}}$ may be written as

$$
\gamma_{m}=\frac{X_{\mathrm{k}}}{X_{\mathrm{d}}}=\frac{\mu_{X}+u_{0.05} \sigma_{X}}{\mu_{X}+u_{p} \sigma_{X}}=\frac{1+u_{0.05} w_{X}}{1+u_{p} w_{X}}, \quad p=\Phi(-0.8 \beta)
$$

where $w_{X}$ denotes coefficients of variation of $X, u_{0.05}$ or $u_{p}$ denotes $5 \%$ - or $p$-fractile of the standardised random variable having the same probability distribution as the resistance $X$. Assuming a log-normal distribution of $X$, then the fractiles $u_{p}$ in equation (8.13) must be taken from the standardised log-normal distribution given in Table 4.2. In the case of a log-normal distribution having the lower bound at zero, equation (8.13) may be written as

$$
\gamma_{m}=\frac{X_{\mathrm{k}}}{X_{\mathrm{d}}}=\frac{\frac{1}{\sqrt{1+w_{X}^{2}}} \exp \left(u_{0.05} \sqrt{\ln \left(1+w_{X}^{2}\right)}\right)}{\frac{1}{\sqrt{1+w_{X}^{2}}} \exp \left(u_{p} \sqrt{\ln \left(1+w_{X}^{2}\right)}\right)} \cong \frac{\exp \left(u_{0.05} \times w_{X}\right)}{\exp \left(u_{p} \times w_{X}\right)}, \quad p=\Phi(-0.8 \beta)
$$

where $u$ denotes now the normal standardised variable, for which detail tables are commonly available (see also Table 4.1 indicating basic data). Note that the approximation indicated in the last expression in equation (8.14) is fully acceptable for small coefficients of variation $w_{X}$ $(<0.2)$. Figures 8.2 and 8.3 show the variation of the partial factor $\gamma_{R}$ of the material property $X$ with the reliability index $\beta$ for selected values of the coefficient of variation $w_{R}$ given for a normal distribution by equation (8.13) (Figure 8.2), and a log-normal distribution by equation (8.14) (Figure 8.3).

\section{Example 8.1}

Consider the strength of construction steel given by the yield point, which has the coefficient of variation $w_{X}$ less than 0,1 . Figure 8.4 shows the dependence of the partial factor $\gamma_{\mathrm{m}}$ on the coefficient of variation $w_{X}$ for three types of distribution functions: a normal $\mathrm{N}$, a log-normal LN with the lower bound at zero $\left(x_{0}=0\right)$ and a log-normal distribution LN with the skewness $\alpha=0,5$ assuming $\beta=3,8$.

Figure 8.4 shows that for a two-parameter log-normal distribution with the lower bound at zero $\left(x_{0}=0\right)$, which is a very popular model for resistance, the partial factor $\gamma_{\mathrm{m}}$ for the coefficient of variation $w_{X}=0,08$ should be about $\gamma_{\mathrm{m}}=1,12$, for a three-parameter lognormal distribution with the skewness 0,5 (a realistic value) $\gamma_{\mathrm{m}}=1,09$. So, considering the material property (without the resistance model uncertainty), the factor $\gamma_{\mathrm{m}}$ may be expected around 1,10 . If the model uncertainty $\gamma_{R \mathrm{~d}}=1,05$ is included, then it follows from (8.12) that

$$
\gamma_{\mathrm{M}}=\gamma_{\mathrm{m}} \gamma_{R \mathrm{~d}}=1.1 \times 1.05=1.155 \approx 1.15
$$

However, due to strain hardening $\gamma_{R \mathrm{~d}}$ is often considered as unity and $\gamma_{\mathrm{M}} \approx \gamma_{\mathrm{m}}$. 


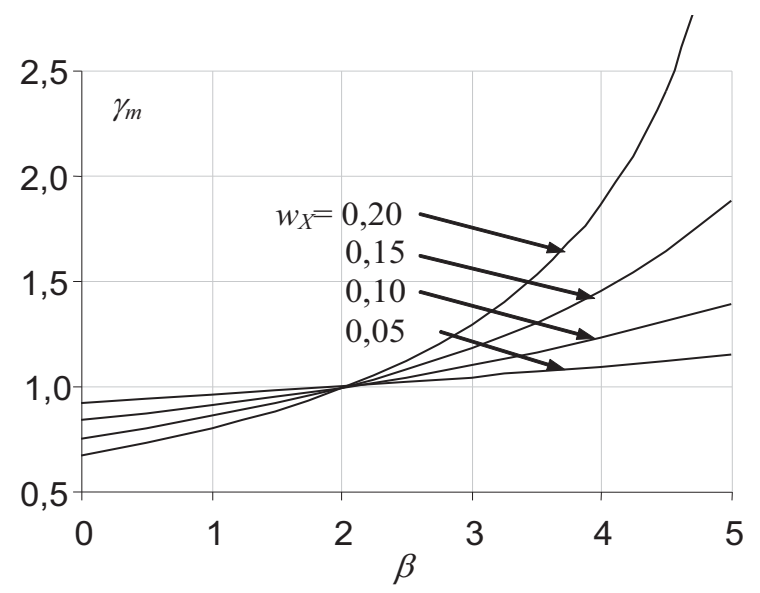

Figure 8.2. Variation of $\gamma_{\mathrm{m}}$ with $\beta$ for selected coefficients of variation $w_{X}=0,05 ; 0,10 ; 0,15$ and 0,20 , and for a normal distribution of $X$.

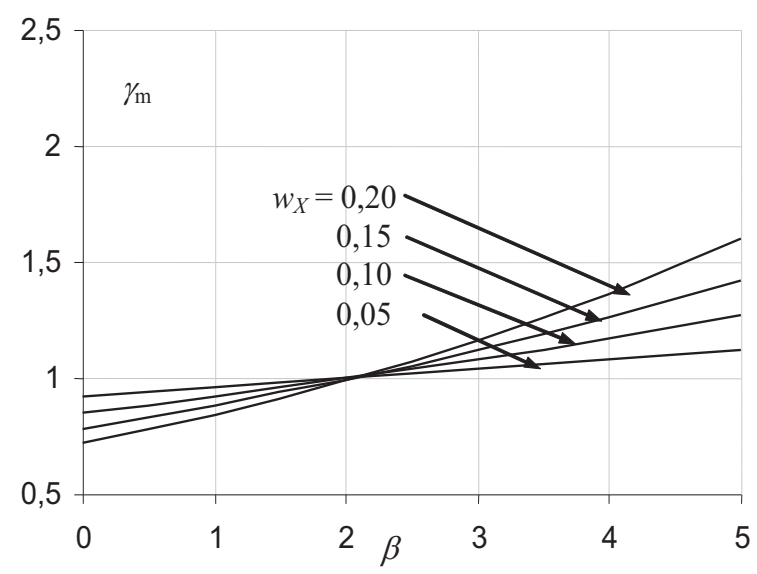

Figure 8.3. Variation of $\gamma_{R}$ with $\beta$ for selected coefficients of variation $X_{R}=0,05 ; 0,10 ; 0,15$ and 0,20 , and for a log-normal distribution of $X$.

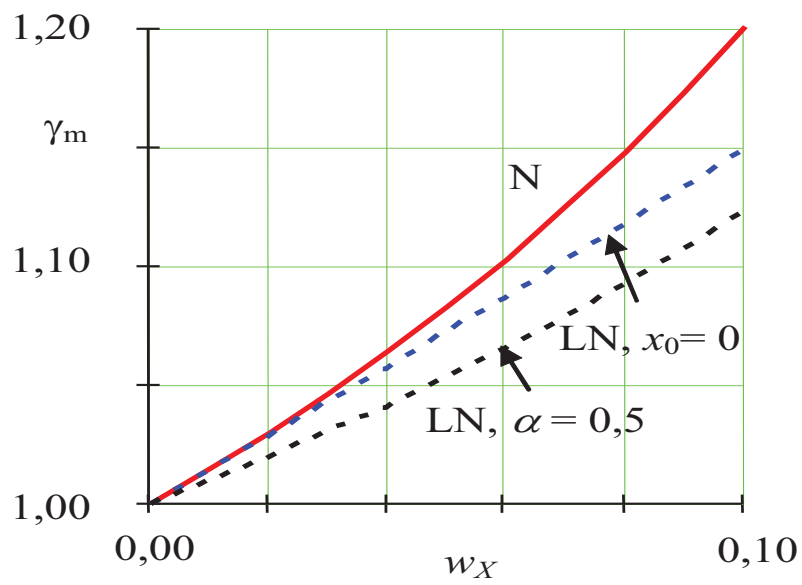

Figure 8.4. Partial factor $\gamma_{\mathrm{m}}$ versus the coefficient of variation $w_{X}$ for normal and log-normal distributions assuming $\beta=3,8$. 
Generally the partial factor $\gamma_{m}$ increases with increasing reliability index $\beta$ value. The increase of $\gamma_{\mathrm{m}}$ is considerably greater in the case of normal distribution (Figure 8.2) than in the case of log-normal distribution (Figure 8.3). The effect of the type of distribution is particularly obvious for coefficients of variation $w_{X}$ greater than 0,10 (Figure 8.4). A considerable effect of the type of distribution on the theoretical value of partial factors can be expected also for other basic variables, in particular for actions.

\subsection{Partial factors for permanent load}

Consider a permanent load $G$ (self-weight) having a normal distribution. It is assumed that the characteristic value $G_{\mathrm{k}}$ of $G$ is defined as the mean $\mu_{G}$ [1], [2] and [16]:

$$
G_{\mathrm{k}}=\mu_{G}
$$

The design value $G_{\mathrm{d}}$ is given (see also documents [1], [2]) as

$$
G_{\mathrm{d}}=\mu_{G}-\alpha_{G} \times \beta \times \sigma_{G}=\mu_{G}+0,7 \times \beta \times \sigma_{G}=\mu_{G}\left(1+0,7 \times \beta \times w_{G}\right)
$$

In equation (8.16) $\mu_{G}$ denotes the mean, $\sigma_{G}$ the standard deviation, $w_{G}$ the coefficient of variation and $\alpha_{G}=-0,7$ the sensitivity factor of $G$.

The partial factor $\gamma_{G}$ of $G$ is given as [1], [2]

$$
\gamma_{g}=G_{\mathrm{d}} / G_{\mathrm{k}}
$$

Taking into account equations (8.15) and (8.16) it follows from (8.17) that

$$
\gamma_{g}=\left(1+0,7 \times \beta \times w_{G}\right)
$$

Figure 4 shows the variation of the partial factor $\gamma_{G}$ with the reliability index $\beta$ for selected values of the coefficient of variation $w_{G}=0,05 ; 0,10 ; 0,15$ and 0,20 .

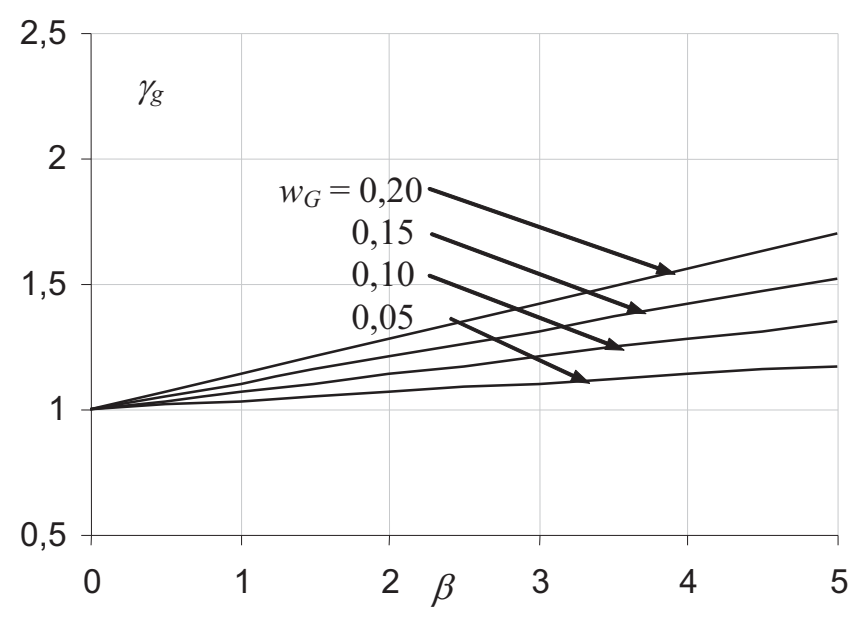

Figure 8.5. Variation of $\gamma_{g}$ with $\beta$ for selected coefficients of variation $w_{G}=0,05 ; 0,10 ; 0,15$ and 0,20 , and for a normal distribution of $G$. 
Note that $\gamma_{G}=1,35$ (recommended in EN 1990 [1]) corresponds approximately to the reliability index $\beta=3,8$ if the coefficient of variation is about 0,1 (the value recommended in EN 1990 [1] was further increased by 5\% to take into account model uncertainty).

It follows from Figures 8.2, 8.3 and 8.5 that less significant variation with $\beta$-values should be generally expected for the partial factor of self-weight $\gamma_{G}$ than for the partial factor of material property $\gamma_{R}$.

\section{Example 8.2}

Consider a permanent load, which has the coefficient of variation $w_{G}=0,1$. It follows from equation (8.18) and Figure 8.5 that for $w_{G}=0,1$ the partial factor $\gamma_{\mathrm{g}}=1,28$. If the model uncertainty $\gamma_{E \mathrm{~d}}=1,05$ is included, then it follows from equation (8.12) that

$$
\gamma_{G}=\gamma_{\mathrm{g}} \gamma_{E \mathrm{~d}}=1.28 \times 1.05=1.33 \approx 1.35
$$

Note that the value $\gamma_{G}=1.35$ is recommended in EN 1990 [1].

\subsection{Partial factors for variable load}

A similar procedure as in the case of the permanent load $G$ can be used for estimation of the partial factors $\gamma_{Q}$ for variable loads $Q$. Assuming the Gumbel distribution the characteristic value is usually defined as 0,98 fractile of annual extremes (or extremes related to a certain basic reference period) and is given as

$$
Q_{\mathrm{k}}=\mu_{Q}\left(1-w_{Q}(0,45+0,78 \ln (-\ln (0,98)))\right)
$$

The design value $Q_{\mathrm{d}}$ related to the design working life or other reference period is given as

$$
Q_{\mathrm{d}}=\mu_{Q}\left(1-w_{Q}\left(0,45-0,78 \alpha_{T} \ln (N)+0,78 \ln \left(-\ln \left(\Phi^{-1}\left(-\alpha_{E} \beta\right)\right)\right)\right)\right.
$$

In equations (8.19) and (8.20) $\mu_{Q}$ denotes the mean, $w_{Q}$ the coefficient of variation of extreme values of $Q$ determined for the basic reference periods (that is, for example 1 or 5 years), $N$ denotes the ratio of the working design life, for example 50 years (or other reference period), and the basic reference period. As an example the period ratio $N=10(=50 / 5)$ is considered below. Finally, $\alpha_{E}=-0,7$ is the sensitivity factor of $Q$ and $\alpha_{T}$ is the time-sensitivity factor given by the ratio $w^{\prime}{ }_{Q} / w_{Q}$ where $w_{Q}^{\prime}$ denotes the coefficient of variation of the timedependent component of $Q$ and $w_{Q}$ denotes the coefficient of variation of the total $Q$. When $Q$ depends on time-dependent components only, $w^{\prime}{ }_{Q}=w_{Q}$ and $\alpha_{T}=1$. Note that the reliability index $\beta$ in equation (8.20) is related to the design working life (for example to the reference period of 50 years) and not to the basic reference period (for example to 1 or 5 years).

The partial factor $\gamma_{Q}$ of $Q$ is given as [1], [2]

$$
\gamma_{Q}=Q_{\mathrm{d}} / Q_{\mathrm{k}}
$$

The partial factor $\gamma_{Q}$ of a variable action $Q$ defined by equation (8.21) depends on five parameters. In addition to $w_{Q}, \alpha_{E}, \beta$ (used also in the case of time-invariant basic variables), the partial factor of variable actions $\gamma_{Q}$ depends also on the period ratio $N$ and on the timesensitivity factor $\alpha_{T}$. Figure 8.6 shows the variation of $\gamma_{Q}$ with the coefficients of variation $w_{Q}$ for selected values of $\beta$ assuming a Gumbel distribution of $Q$, and the period ratio $N=10$ (the reference period 10 times greater than the basic reference period) and the time-sensitivity factor $\alpha_{T}=1$ (no time-independent components). Figure 8.7 shows the variation of $\gamma_{Q}$ with the reliability index $\beta$ for selected values of the coefficients of variation $w_{Q}$ assuming again a Gumbel distribution of $Q, N=10$ and $\alpha_{T}=1$. 


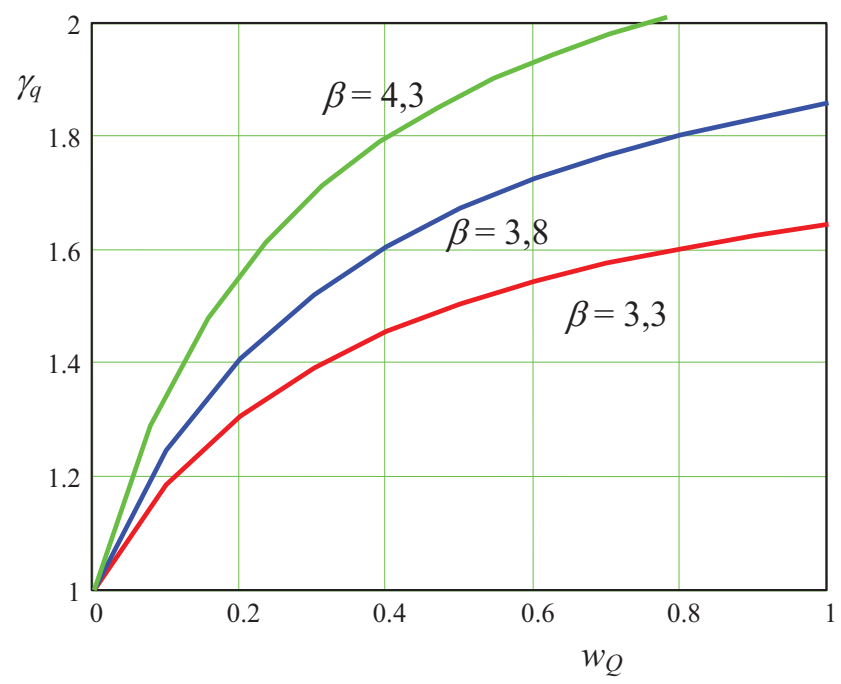

Figure 8.6. Variation of $\gamma_{Q}$ with the coefficients of variation $w_{Q}$ for selected values of $\beta$ assuming a Gumbel distribution of $Q, N=10$ and $\alpha_{T}=1$.

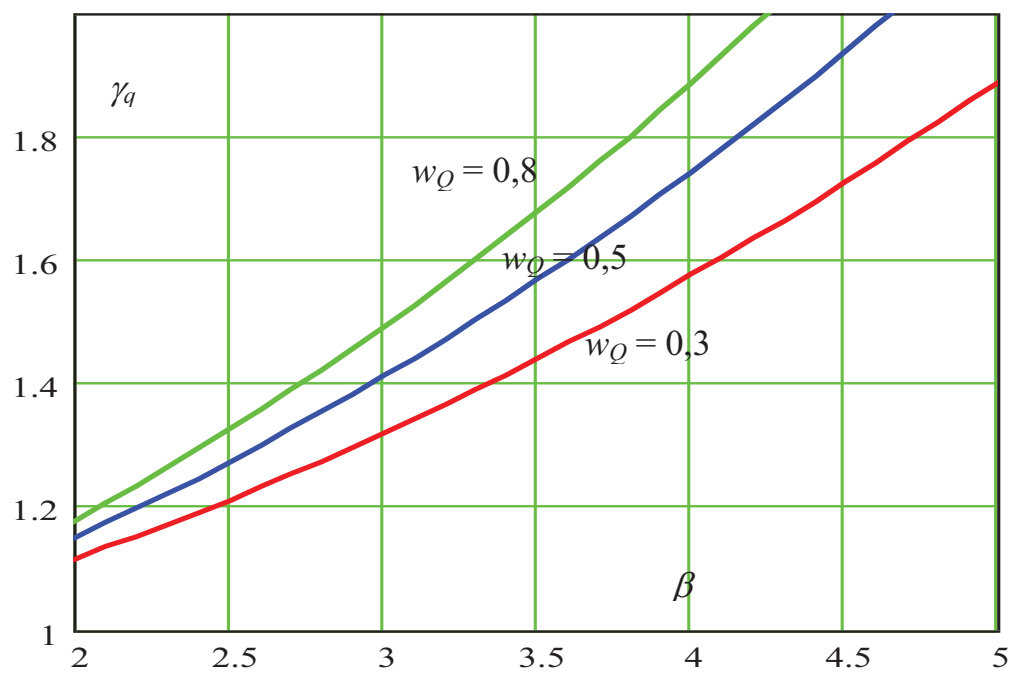

Figure 8.7. Variation of $\gamma_{Q}$ with the reliability index $\beta$ for selected values of the coefficients of variation $w_{Q}$ assuming a Gumbel distribution of $Q, N=10$ and $\alpha_{T}=1$.

It should be noted that the time-variant component may have a considerably lower variability than the total action $Q$ and, therefore, a reduced coefficient of variation should be considered in equation (8.20) for estimating time-variant effects $\left(\alpha_{T}<1\right)$. Consequently, the predicted design value $Q_{\mathrm{d}}$ and the partial factor $\gamma_{Q}$ would decrease. Without going into details, it appears that the value $\gamma_{Q}=1,5$, recommended in EN 1990 [1], is a reasonable approximation corresponding to the reliability index $\beta=3,8$, to the coefficient of variation $w_{Q}=0,3$ (that may be considered as a reduced coefficient of variation of the extremes of $Q$ ) and the period ratio $N=10$ (the reference period 10 times greater than the basic reference period). 


\section{Example 8.3}

Consider a variable load, which has the coefficient of variation $w_{Q}=0,3$. Assume that $N=10$ and $\alpha_{T}=1$ (for example an imposed load). It follows from equations (8.19), (8.20) and (8.21) that the partial factor $\gamma_{\mathrm{q}}=1,48$ (see also Figures 8.6 and 8.8). If the model uncertainty $\gamma_{E \mathrm{~d}}=1,05$ is accepted, then it follows from equation (8.12) that

$$
\gamma_{Q}=\gamma_{\mathrm{q}} \gamma_{E \mathrm{~d}}=1.48 \times 1.05=1.54 \approx 1.5
$$

Note that the value $\gamma_{Q}=1.5$ is recommended in EN 1990 [1].

\subsection{Partial factors for wind action}

A typical example of a variable load $Q$ affected significantly by time-invariant components is the wind action. The wind pressure (or force) may be generally written as [27]

$$
q=0,5 \rho v^{2} c_{\mathrm{r}} c_{\mathrm{a}} c_{\mathrm{g}} c_{\mathrm{d}}=Q_{\mathrm{ref}} c_{\mathrm{r}} c_{\mathrm{a}} c_{\mathrm{g}} c_{\mathrm{d}}
$$

where $Q_{\text {ref }}=0,5 \rho v^{2}$ denotes the time-variant reference pressure dependent on the air density $\rho$ (deterministic quantity equal to $1,25 \mathrm{~kg} / \mathrm{m}^{3}$ ) and the wind speed $v$ (having the characteristic value based on the annual extremes, for example $26 \mathrm{~m} / \mathrm{s}$ ). The roughness factor $c_{\mathrm{r}}$, aerodynamic shape factor $c_{\mathrm{a}}$, gust factor $c_{\mathrm{g}}$ and dynamic factor $c_{\mathrm{d}}$ may be considered as timeinvariant random variables depending on structural and environmental conditions.

Table 8.1 shows indicative parameters of the variables entering equation (8.22), which are adopted from JCSS [27]. However, the parameters are in general significantly dependent on particular conditions. Moreover, the indications provided in literature (mentioned in [27]) are rather inconclusive. The following results should therefore be considered as indicative findings only. Nevertheless, the submitted general procedures for determining the partial factor $\gamma_{Q}$ may be applied for more specific characteristics of wind components, a given structure and environmental conditions.

Table 8.1. Indicative parameters of the wind components.

\begin{tabular}{|l|l|c|c|}
\hline Symbol & Name of the variable & Relative mean & Coefficient of variation $w$ \\
\hline$Q_{\text {ref }}$ & Annual pressure extreme & 0,8 & 0,25 \\
\hline$c_{\mathrm{r}}$ & Roughness factor & 0,8 & 0,15 \\
\hline$c_{\mathrm{a}}$ & Aerodynamic shape factor & 1,0 & 0,20 \\
\hline$c_{\mathrm{g}}$ & Gust factor & 1,0 & 0,15 \\
\hline$c_{\mathrm{d}}$ & Dynamic factor & 1,0 & 0,15 \\
\hline
\end{tabular}

Considering the parameters of wind components indicated in Table 8.1, the resulting coefficient of annual wind action (wind pressure or force) is about $w_{Q}=0,4$ (the expected range is from 0,3 to 0,6 ) and the sensitivity factors $\alpha_{T}=0,6$ (the expected range is from 0,5 to $0,8)$. It is assumed that both the reference pressure $Q_{\text {ref }}$ and the wind action $q$ may be approximately described by Gumbel distribution. 
Figure 8.8 shows the variation of $\gamma_{Q}$ with the coefficient of variation $w_{Q}$ for the reliability index $\beta=3,8, N=50$ and $\alpha_{T}=0,5 ; 0,6 ; 0,8$ and 1,0 (the extreme case indicated in Figure 8.8 by a dashed line) assuming approximately a Gumbel distribution for both $Q_{\text {ref }}$ and $q$. It follows from Figure 8.8 that the partial factor $\gamma_{Q}$ may be significantly affected by the time-sensitivity factor $\alpha_{T}$, particularly for the coefficient of variation $V_{Q}$ expected for annual extremes of wind pressure (around 0,4). Note that for the coefficient of variation $w_{Q}=0,4$ and the sensitivity factor $\alpha_{T}=0,6$ the partial factor $\gamma_{Q}$ is about 1,6 , which is a slightly greater value than the value 1,5 recommended in EN. However, taking into account the expected reduction of the actual mean of the reference pressure $Q_{\text {ref }}$ and the roughness factor $c_{\mathrm{r}}$ indicated in Table 1 (given in [27]), the partial factor $\gamma_{Q}=1,5$ seems to be a reasonable value. Note that for $w_{Q}=0,4$ and the time-sensitivity factor $\alpha_{T}=1,0$ (valid in case of no timeindependent components) the partial factor is $\gamma_{Q}=1,85$ (see Figure 8.8). Obviously, further research is needed to specify plausible statistical parameters of wind actions.

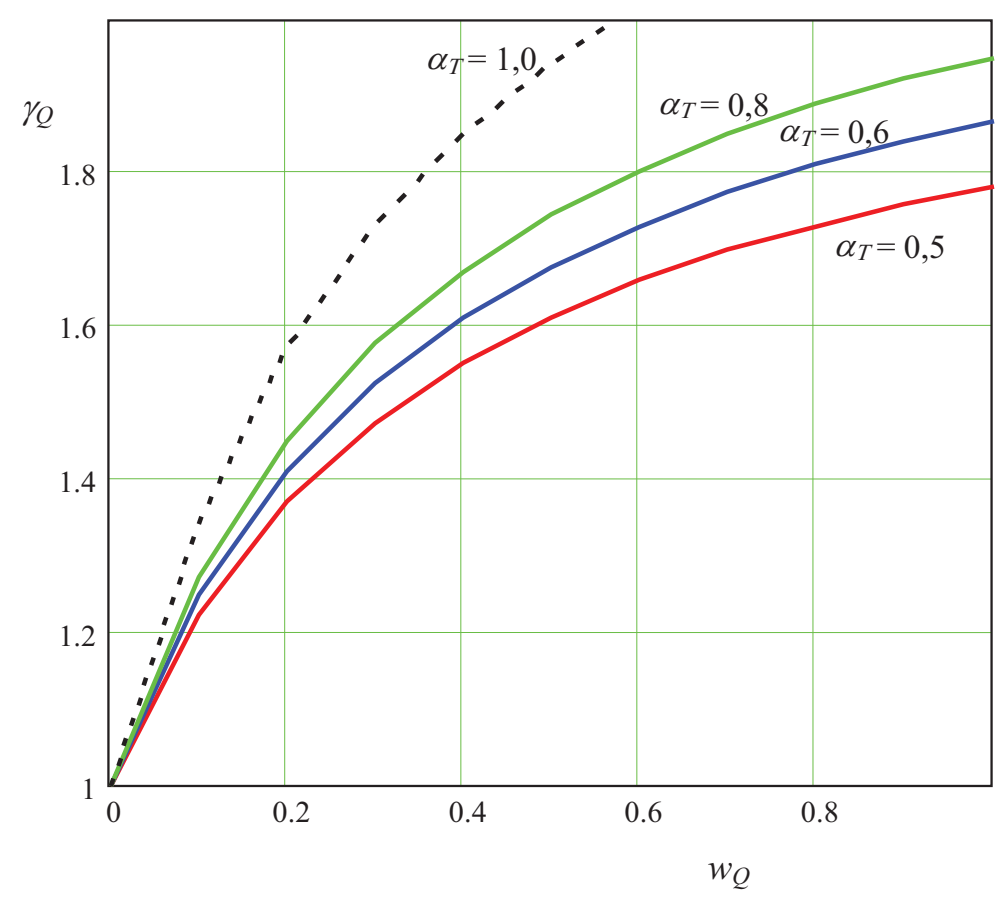

Figure 8.8. Variation of $\gamma_{Q}$ with the coefficient of variation $w_{Q}$ for the reliability index $\beta=3,8$, $N=50$ and $\alpha_{T}=0,5 ; 0,6 ; 0,8$ and 1,0, assuming a Gumbel distribution for $Q_{\text {ref }}$ and $q$.

\section{Example 8.4}

Consider a wind action, which has the coefficient of variation $w_{Q}=0,3$. Assume that $N$ $=50$ and $\alpha_{T}=0,4$. It follows from equations (8.19), (8.20) and (8.21) (see also Figures 8.8) that the partial factor $\gamma_{Q}=1,55$. If the model uncertainty $\gamma_{E \mathrm{~d}}=1,05$ is included, then it follows from equation (8.12) that

$$
\gamma_{Q}=\gamma_{\mathrm{q}} \gamma_{E \mathrm{~d}}=1.54 \times 1.05=1.61 \approx 1.6
$$


Note that the value $\gamma_{Q}=1.5$ is recommended in EN 1990 [1] generally for all variable actions.

\subsection{Concluding remarks}

Elementary methods of structural reliability can be used to assess the partial factors and other reliability elements in the fundamental case of two random variables when the limit state function is formulated as the difference between the resulting structural resistance and load effect. More sophisticated theoretical principles of the First Order Reliability Method (FORM) are applicable to a general case of more basic variables.

The basic principles of FORM provide operational techniques that can be used for estimating the design points and partial factors of basic variables in general, multivariate reliability tasks. However, the assessment of various reliability elements in the new structural design codes is partly based on historical and past experience. Such experience may depend on local conditions including climatic actions and traditionally used construction materials and, consequently, might be considerably diverse in different countries. That is why a number of reliability elements and parameters in the present suite of European standards are open for national choice.

The partial factor $\gamma_{Q}$ for wind pressure is dependent on the time-sensitivity factor $\alpha_{T}$, particularly for the coefficients of variation $w_{Q}$ within the expected range for annual extremes of wind pressure (around 0,4). For $w_{Q}=0,4$ and the sensitivity factor $\alpha_{T}=0,5$ the theoretical value of the partial factor $\gamma_{Q}$ is relatively high, about 1,6 . However, taking into account the expected reduction of the actual mean of the reference pressure $Q_{\text {ref }}$ and the roughness factor $c_{\mathrm{r}}$ indicated in the JCSS materials [27], the partial factor $\gamma_{Q}=1,5$ recommended in EN 1990 [1] seems to be an adequate value. Further research is needed to specify plausible statistical parameters of wind actions. 


\subsection{General}

Structures of buildings and other engineering works commonly consist of a system of structural elements (or members). Failure of such a structure may have several modes that should be considered when analysing the overall structural reliability. However, even in the case of one structural member (say a beam or a column), several failure modes (limit state functions) have to be taken into account. For example, a continuous beam may fail due to positive or negative moments or due to shear. This chapter provides a short summary of the basic principles of system reliability. A more detailed comprehensive text may be found in a number of recent publications [18, 21, 22].

In general, the reliability of a structural system depends on the reliability of its elements and relevant failure modes. As a rule there is a high correlation between the properties of elements in different parts of the structure. In some cases loads may also be mutually dependent. In addition, the limit states for the whole structure such as the overall deflection or foundation settlement may be significant.

When all the different failure modes are identified, a 'fault-tree' or an -tree' modelling failure modes can be established. Basic principles of system reliability analysis are illustrated by a simple example of a plane frame.

\section{Example 9.1}

Consider the simple portal frame shown in Figure 9.1. The frame is exposed to the horizontal force $H$ and the vertical loads $G+Q$.

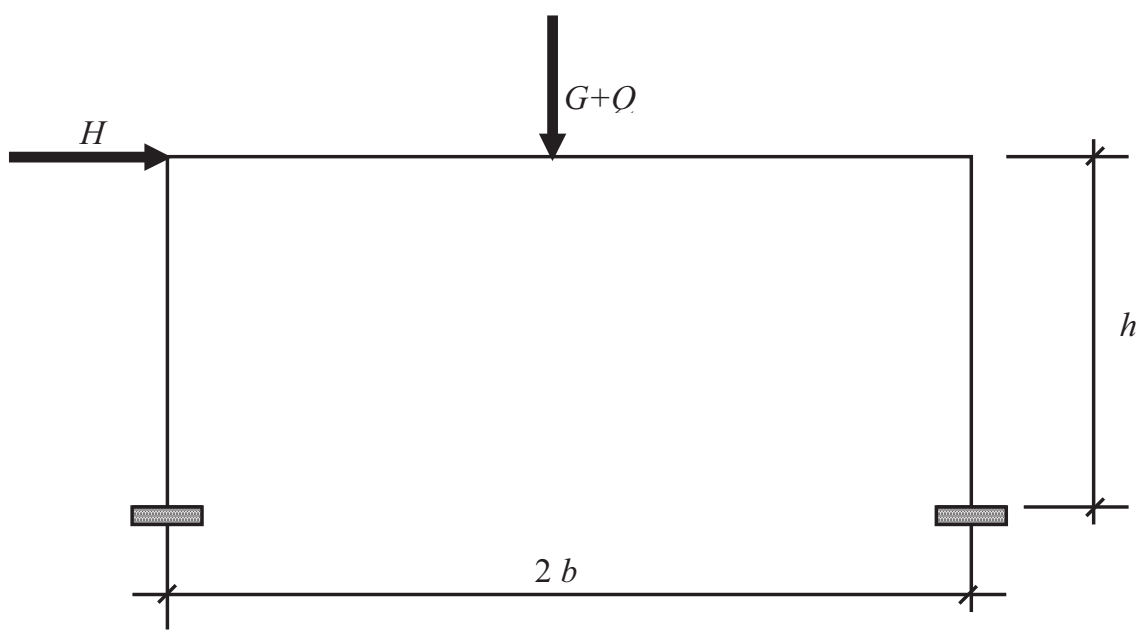

Figure 9.1. A portal frame.

Assuming plastic behaviour, the frame has three possible modes of collapse. In each path different plastic hinges will be formed as follows:

a) Sway mode: Plastic hinges formed at sections 1, 2, 4 and 5;

b) Beam mode: Plastic hinges at sections 2, 3, and 4,

c) Combined mode: Plastic hinges at sections 1, 3, 4 and 5 . 
Figure 9.2 indicates the abovementioned failure modes and appropriate plastic hinges at sections 1 to 5 .
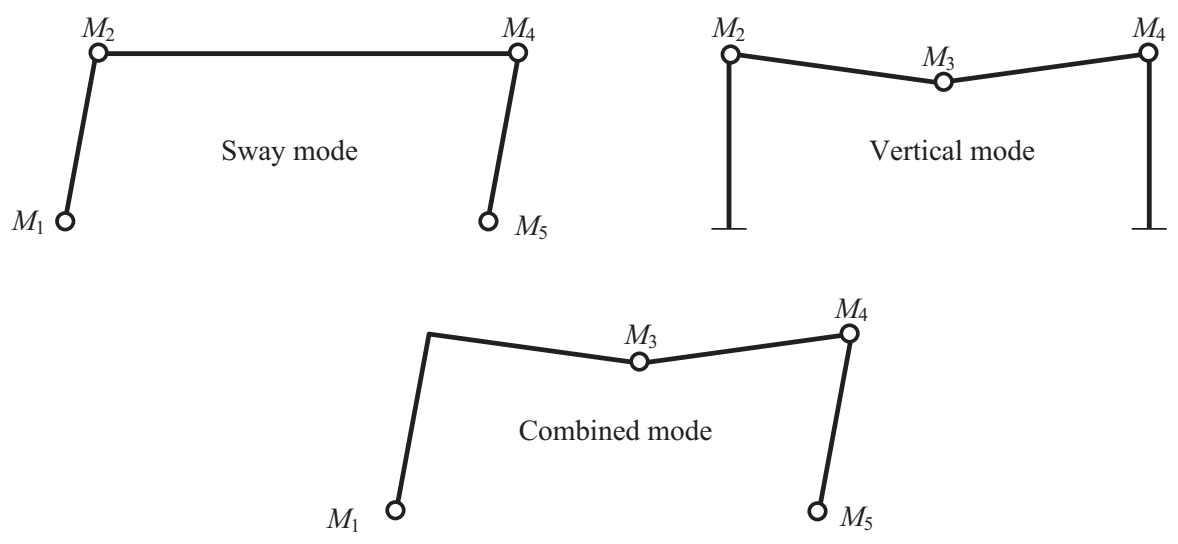

Figure 9.2. Failure modes.

A fault tree describing the failure of the portal frame is indicated in Figure 9.3 together with the deformed shape of the frame due to the above-mentioned failure modes.

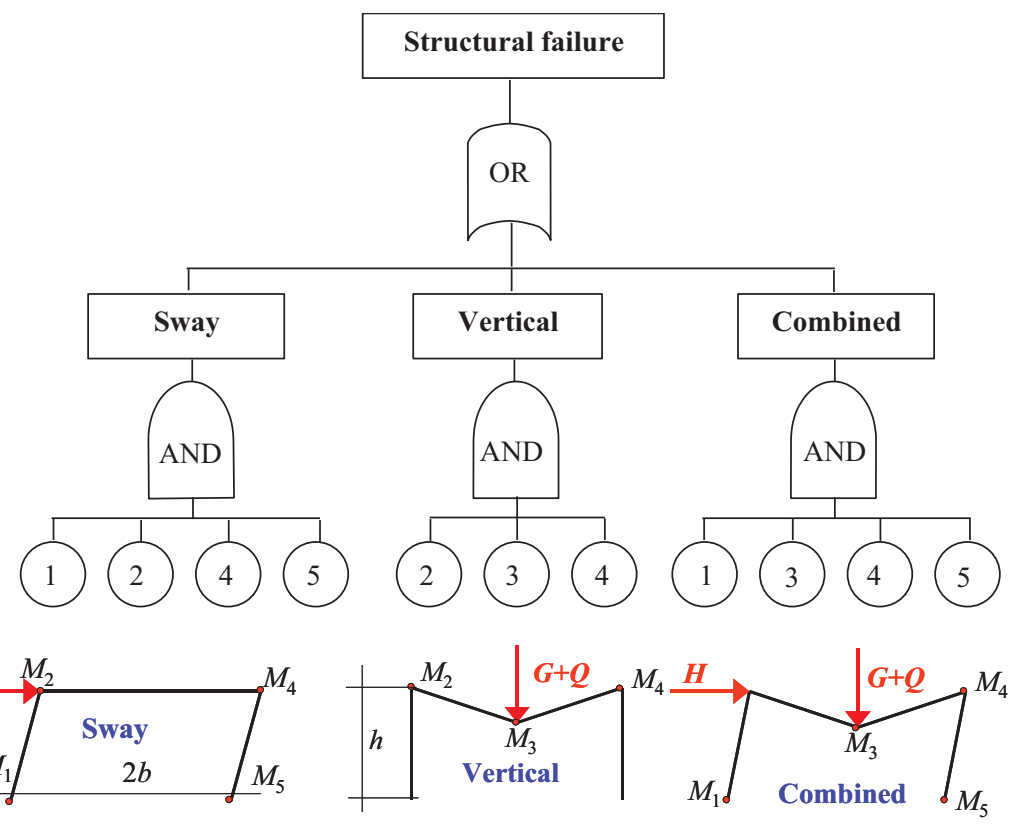

Figure 9.3. A fault tree describing the failure of a plane frame.

Figure 9.3 clearly shows logical operators "AND", applicable in the case of the simultaneous development of appropriate plastic hinges in each failure mode, and "OR", applicable in the union of different failure modes. 
As indicated in Figure 9.3, any of the $n$ failure modes $(n=3$, in example 9.1 and Figure 9.3) leads to structural failure (a series system). The failure is then the union of failures $F_{i}$ due to modes $i=1,2, \ldots n$. Then the probability of failure $F_{S}$ of the structure can be written as

$$
P_{\mathrm{f}}=\mathrm{P}\left(F_{S}\right)=P\left(F_{1} \cup F_{2} \cup \ldots \cup F_{n}\right)
$$

where $F_{i}$ denotes the failure due to mode $i(i=1,2, \ldots n)$. For each mode $i$, a certain number of $m$ nodes or structural elements have to fail simultaneously (in the example 9.1 plastic hinges are developed). So the probability of failure of each mode, $F_{i}$, will be:

$$
P_{\mathrm{f}}\left(F_{i}\right)=\mathrm{P}\left(F_{i 1} \cap F_{i 2} \cap \ldots \cap F_{i m}\right)
$$

where $F_{i j}$ is the event failure of the $j$-th element or node in the $i$-th failure mode. To reach the collapse of the structure in the mode $i, m$ elements or nodes must fail.

In general, the structure is idealised as a series system (described by equation (9.1)), or a parallel system (described by equation (9.2)), or the combination of both.

\subsection{Parallel system}

In the parallel system, the elements are so interconnected that the failure of one or more of the elements does not mean the failure of the whole structure. Such a structure is called a redundant structure. This redundancy can be active, when the redundant members can activate before the limit state of any member is reached, or passive, when the redundant members act only when a limit state is reached in some member.

It should be taken into account that any hyperstatic structure is not necessarily a parallel system: if the elements are brittle, the failure of any of them can lead to a new distribution of stresses so that new failures are reached immediately.

The failure probability of a pure parallel system with $m$ components is given by:

$$
P_{\mathrm{f} \mathrm{sys}}=\mathrm{P}\left(\bigcap_{1}^{\mathrm{m}} F_{j}\right)=\mathrm{P}\left[\bigcap_{1}^{m}\left(\mathrm{G}_{j}<0\right)\right]
$$

where $F_{j}$ is the failure of the $j$-th component with $G_{j}$ limit state function. Thus:

$$
\mathrm{P}\left(F_{j}\right)=\mathrm{P}\left(G_{j}<0\right) \approx \Phi(-\beta)
$$

The probability of failure of the system by FORM is given by:

$$
P_{\mathrm{f} \mathrm{sys}}=\Phi_{\mathrm{m}}(-\boldsymbol{\beta} ; \boldsymbol{\rho})
$$

where $\Phi_{\mathrm{m}}$ is the multi-dimensional standard normal distribution, $\boldsymbol{\beta}$ is the vector of the component reliability indices and $\rho$ the $m \times m$ correlation matrix of the indices, given by

$$
\rho_{j k}=\sum_{i} \alpha_{i j} \alpha_{i k} ; j, k=1,2, \ldots, m
$$

and $\alpha_{i j}$ is the sensitivity factor of the $i$-th variable in the $j$-th component limit state function.

It is a demanding task to calculate the probability of failure of the system. An upper bound of this probability can be obtained as:

$$
P_{\mathrm{f} \mathrm{sys}}=\underset{j, k=1}{m}\left\lfloor P\left(F_{j} \cap F_{k}\right)\right\rfloor
$$

A simple approximation for only two elements:

$$
P_{\mathrm{f} \mathrm{sys}}=\Phi\left(-\beta_{1}\right) \Phi\left(-\beta_{2}^{*}\right) ; \beta_{2}^{*}=\left(\beta_{2}-\rho \beta_{1}\right) / \sqrt{1-\rho^{2}}
$$

\subsection{Series system}

A series system is where the failure of any element leads to the collapse of the structure. It is called the 'weakest link'. Any statically determined structure is a series system. 
In a similar manner that was shown in the previous section, the failure probability in a pure series system with $m$ components is given by:

$$
P_{\mathrm{fsys}}=P\left(\bigcup_{1}^{\mathrm{m}} F_{j}\right)
$$

The probability of failure of the system given by FORM is:

$$
P_{\mathrm{f} \mathrm{sys}}=1-\Phi_{\mathrm{m}}(\boldsymbol{\beta} ; \boldsymbol{\rho})
$$

Simple bounds to this probability are given by:

$$
\underset{1}{\max }\left[P\left(F_{j}\right)\right] \leq P_{\mathrm{f} \text { sys }} \leq \operatorname{Min}\left[\sum_{1}^{m} P\left(G_{j}\right), 1\right] .
$$

These bounds are usually too wide. Methods to find more precise bounds for series, parallel and hybrid systems can be found in $[18,21,22]$.

\section{Example 9.2}

Consider the previous Example 9.1 in detail. Using the principle of virtual work the failure modes shown in Figure 9.2 can be described by the following limit state functions:

Sway failure mode $\mathrm{F}_{1}: \mathrm{g}_{1}(X)=M_{1}+M_{2}+M_{4}+M_{5}-h H$

Vertical failure mode $\mathrm{F}_{2}: \mathrm{g}_{2}(X)=M_{2}+2 M_{3}+M_{4}-b(G+Q)$

Combined failure mode $\mathrm{F}_{3}: \mathrm{g}_{3}(X)=M_{1}+2 M_{3}+2 M_{4}+M_{5}-h H-b(G+Q)$

It is assumed that the plastic moments $M_{i}(i=1,2,3,4,5)$ at all hinges $(i=1,2,3,4$, 5) have the same characteristic value $M_{\mathrm{k}}$ (say $100 \mathrm{kNm}$ ). Considering now the combined failure mode, the characteristic value $M_{\mathrm{k}}$ and the characteristic values of actions $G_{\mathrm{k}}, Q_{\mathrm{k}}$, and $H_{\mathrm{k}}$ may be related as follows:

Sway failure mode:

$h \gamma_{H} \psi_{0} H_{\mathrm{k}}=4 M_{\mathrm{k}} / \gamma_{\mathrm{M}}$

Vertical failure mode:

$$
b\left(\gamma_{G} G_{\mathrm{k}}+\gamma_{Q} Q_{\mathrm{k}}\right)=4 M_{\mathrm{k}} / \gamma_{\mathrm{M}}
$$

Combined failure mode:

$$
b\left(\gamma_{G} G_{\mathrm{k}}+\gamma_{Q} Q_{\mathrm{k}}\right)+h \gamma_{H} \psi_{0} H_{\mathrm{k}}=6 M_{\mathrm{k}} / \gamma_{\mathrm{M}}
$$

Here $\gamma_{H}, \gamma_{G}$, and $\gamma_{Q}$ denote the partial factors and $\psi_{0}$ the combination factor. For the given actions $G_{\mathrm{k}}, Q_{\mathrm{k}}$ and $H_{\mathrm{k}}$ the required resistance moment $M_{\mathrm{k}}$ is given as the maximum value obtained from the above relationships.

In the following parametric study two additional parameters are introduced:

$-\chi$ (the interval $<0,1>$ ) denoting the ratio of variable actions $\left(Q_{\mathrm{k}}+H_{\mathrm{k}}\right)$ and the total action $\left(G_{\mathrm{k}}+Q_{\mathrm{k}}+H_{\mathrm{k}}\right)$

$$
\chi=\left(Q_{\mathrm{k}}+H_{\mathrm{k}}\right) /\left(G_{\mathrm{k}}+\mathrm{Q}_{\mathrm{k}}+H_{\mathrm{k}}\right)
$$

$-k$ (around 1) denoting the ratio of variable actions $H_{\mathrm{k}}$ and $Q_{\mathrm{k}}$

$$
k=H_{\mathrm{k}} / \mathrm{Q}_{\mathrm{k}}
$$

In the reliability analysis the following probabilistic models (covering implicitly model uncertainties) are considered for basic variables:

$$
\begin{gathered}
M_{i}: \operatorname{LN}\left(\mu_{M}, 0,1 \mu_{M}\right) \\
G: \operatorname{N}\left(G_{\mathrm{k}}, 0,1 G_{\mathrm{k}}\right) \\
Q: \operatorname{LN}\left(0,2 Q_{\mathrm{k}}, 0,35 Q_{\mathrm{k}}\right) \\
H: \operatorname{LN}\left(0,3 H_{\mathrm{k}}, 0,5 H_{\mathrm{k}}\right)
\end{gathered}
$$

The mean of the plastic moments $M_{i}$ is given as $\mu_{M}=M_{\mathrm{k}} \mu_{R} / R_{\mathrm{k}}\left(=M_{\mathrm{k}} \times 280 / 235\right)$, where $\mu_{R}=R_{\mathrm{k}} \times 280 / 235$ is an approximation of the mean strength for the steel S 235. The geometric data $h=8 \mathrm{~m}$ and $b=5 \mathrm{~m}$ are considered as deterministic quantities.

The failure probability of the series system is given as 


$$
P_{\mathrm{f}}=\mathrm{P}\left\{F_{1} \cup F_{2} \cup F_{3}\right\} \leq \mathrm{P}\left\{F_{1}\right\}+\mathrm{P}\left\{F_{2}\right\}+\mathrm{P}\left\{F_{3}\right\}
$$

The probabilities shown in Table 9.1 have been obtained using the program SYSREL (the input file is shown in Appendix). The following parameters were assumed: $\gamma_{G}=1.35, \gamma_{Q}$ $=\gamma_{H}=1.5, \psi_{0}=0.6, \chi=0.6, k=0,5$, and the mutual coefficient of correlation between $M_{2}, M_{3}$ and $M_{4}$ equal to 0.5 (correlations of the plastic moments of the beam). Under these conditions the vertical mode is the most critical failure mode (failure probabilities of other two modes are negligible). However, the significance of individual failure modes depends on the loading conditions including the parameters $\chi$ and $k$.

Table 9.1. Failure mode probabilities.

\begin{tabular}{|l|c|c|c|c|}
\hline & $\begin{array}{c}\text { Sway mode } \\
\mathrm{P}\left\{F_{1}\right\}\end{array}$ & $\begin{array}{c}\text { Vertical mode } \\
\mathrm{P}\left\{F_{2}\right\}\end{array}$ & $\begin{array}{c}\text { Combined mode } \\
\mathrm{P}\left\{F_{3}\right\}\end{array}$ & Union $P_{\mathrm{f}}$ \\
\hline$\beta$ SORM & 6.002 & 4.332 & 5.786 & 4.332 \\
\hline Failure probability & $9.74510^{-10}$ & $7.38810^{-06}$ & $3.60410^{-09}$ & $7.38810^{-06}$ \\
\hline
\end{tabular}

The parametric study for the parameter $\chi$ within the interval $<0,1>$, shown in Figure 9.4, indicates the variation of the reliability index $\beta$ with the parameter $\chi$ (the ratio of the variable and total actions) on the structural reliability.

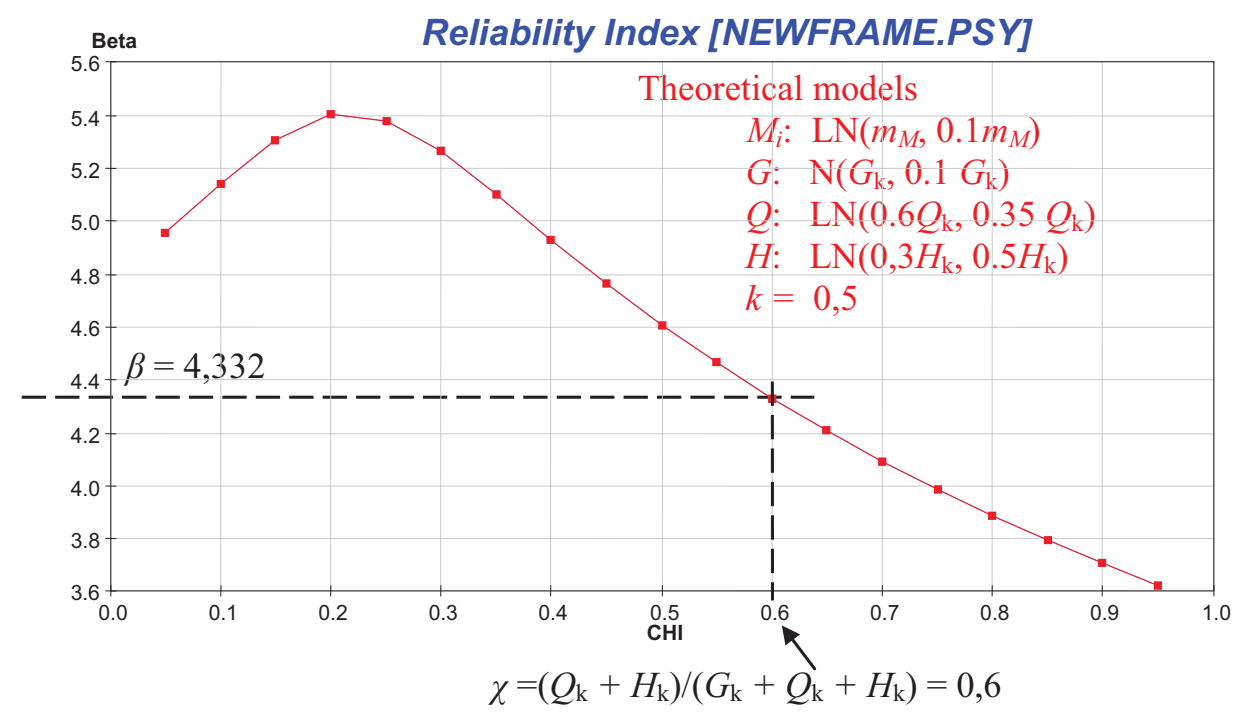

Figure 9.4. Variation of the reliability index $\beta$ with the load parameter $\chi$.

It follows from Figure 9.4 that the reliability index $\beta$ is strongly dependent on the load parameter $\chi$. For the practical range $0<\chi<0,7$ the index $\beta$ is within the range from 4 to 5,4. Figure 9.4 is, however, valid for the indicated theoretical models of basic variables and other abovementioned assumptions only. The variation of the reliability index $\beta$ with the load ratio $\chi$ may be considerably different for other assumptions and the load parameter $k$. In particular, increasing the parameter $k$ (and horizontal force $H$ ) may significantly affect the reliability index $\beta$. 


\section{Appendix. SYSREL input file for the example of a series system.}

Limit state functions/Characteristic actions/The mean actions/Parameters of actions and plastic moments

$\operatorname{FLIM}(1)\{$ Sway mode $\} \quad=\mathrm{M} 1+\mathrm{M} 2+\mathrm{M} 4+\mathrm{M} 5-\mathrm{h} * \mathrm{H}$

$\operatorname{FLIM}(2)\{$ Vertical mode $\}=\mathrm{M} 2+2 * \mathrm{M} 3+\mathrm{M} 4-\mathrm{b} *(\mathrm{G}+\mathrm{Q})$

FLIM(3) $\{$ Combined mode $\}=\mathrm{M} 1+2 * \mathrm{M} 3+2 * \mathrm{M} 4+\mathrm{M} 5-\mathrm{h} * \mathrm{H}-\mathrm{b} *(\mathrm{G}+\mathrm{Q})$

DEFFUNC(4)() $\{$ The characteristic value $\mathrm{Qk}$ as a function of $\mathrm{CHI}\}=\mathrm{CHI} * \mathrm{Gk} /((1+\mathrm{k}) *(1-\mathrm{CHI}))$

DEFFUNC(5)() $\{$ The characteristic value $\mathrm{Hk}$ as a function of $\mathrm{CHI}\}=\mathrm{FUNC}(4) * \mathrm{k}$

DEFFUNC(6)() \{The Md1 as a function of $\mathrm{Gk}\}=(\mathrm{h} * \mathrm{FUNC}(5) * \mathrm{gH}) / 4$

DEFFUNC(7)() $\{$ The Md 2 as a function of $\mathrm{Gk}\}=\mathrm{b} *(\mathrm{gG} * \mathrm{Gk}+\mathrm{gQ} * \mathrm{FUNC}(4)) / 4$

DEFFUNC(8)() \{The Md3 as a function of

$\mathrm{Gk}\}=(\mathrm{h} * \mathrm{FUNC}(5) * \mathrm{gH} * \mathrm{psiH}+\mathrm{b} *(\mathrm{gG} * \mathrm{Gk}+\mathrm{gQ} * \mathrm{FUNC}(4))) / 6$

DEFFUNC $(9)()\{$ The mean of $\mathrm{M}$ as the maximum $\}=$

$(280 / 235) * \mathrm{gM}^{*} \mathrm{MAX}(\mathrm{MAX}(\mathrm{FUNC}(6), \mathrm{FUNC}(7)), \mathrm{FUNC}(8))$

DEFFUNC (10)() $\{$ The mean of $\mathrm{G}\}=\mathrm{Gk} * \mathrm{mG}$

DEFFUNC(11)() $\{$ The mean of Q $\}=F U N C(4) * m Q$

DEFFUNC(12)() $\{$ The mean of $\mathrm{H}\}=\mathrm{FUNC}(5) * \mathrm{mH}$

$\mathrm{RF} 07(1)=\mathrm{FUNC}(10)$

$\mathrm{RF} 07(2)=\operatorname{covG} * \mathrm{FUNC}(10)$

$\mathrm{RF} 07(3)=\mathrm{FUNC}(11) * \mathrm{EXP}\left(-\mathrm{LN}\left(1+(\operatorname{covQ})^{\wedge} 2\right) / 2\right)$

$\mathrm{RF} 07(4)=\operatorname{SQRT}\left(\mathrm{LN}\left(1+(\operatorname{covQ})^{\wedge} 2\right)\right)$

$\mathrm{RF} 07(7)=\mathrm{FUNC}(12)^{*} \mathrm{EXP}\left(-\mathrm{LN}\left(1+(\operatorname{covH})^{\wedge} 2\right) / 2\right)$

$\mathrm{RF} 07(8)=\operatorname{SQRT}\left(\mathrm{LN}\left(1+(\operatorname{covH})^{\wedge} 2\right)\right)$

$\mathrm{RF} 07(5)=1.0 * \mathrm{FUNC}(9) * \operatorname{EXP}\left(-\mathrm{LN}\left(1+(\operatorname{cov} \mathrm{M}){ }^{\wedge} 2\right) / 2\right)$

$\mathrm{RF} 07(6)=\operatorname{SQRT}\left(\mathrm{LN}\left(1+(\operatorname{covM})^{\wedge} 2\right)\right)$ 


\subsection{General procedure}

A number of national standards and some parts of newly developing European standards for structural design often refer to methods of risk assessment, particularly in the case of structures exposed to accidental actions when the consequences of adverse events are significant. Accidental design situations seem to occur more and more frequently and extents of unfavourable events are of increasing importance. However, up to now no internationally accepted document for the risk analysis and risk evaluation of civil engineering systems is available.

It appears that the standardisation of general principles and rules for the risk assessment of civil engineering systems including tunnels is of the utmost importance. In December 2004 the International Standard Organisation ISO, technical committee TC98, subcommittee SC2 established a new working group responsible for developing a new document on the Risk Management of Civil Engineering Systems. The new International Standard should provide a common methodology and clearly defined terminology that would improve not only the common practice of risk assessment, but also risk communication as an extremely important component of the whole risk management. The aim of the submitted text is to describe important aspects of risk assessment that are applicable to various civil engineering systems.

General principles of risk assessment and the common tools applied for analysing the risk of civil engineering systems described in this informative text are based on the common concepts presented in documents [1] to [10]. The main parts of risk assessment and the basic terminology used in this text are indicated in Figure 10.1 (adopted from [10]). It follows from Figure 10.1 that the risk assessment of a system is an important part of the whole risk management. The risk assessment consists of risk analysis and risk evaluation.

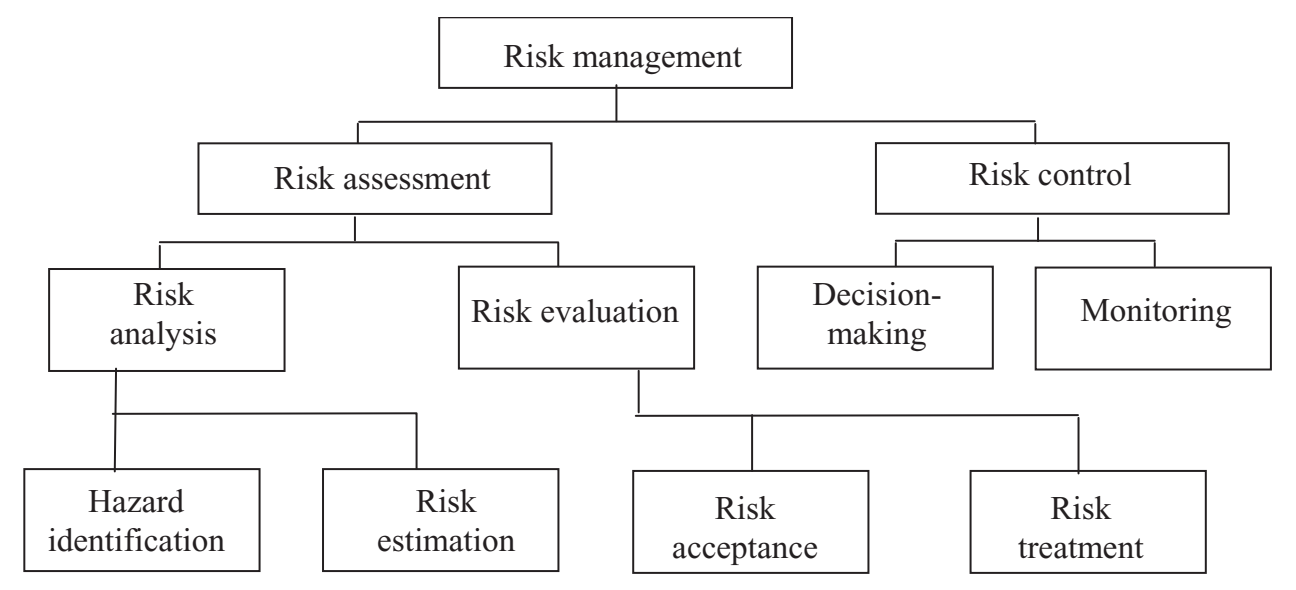

Figure 10.1. A framework for risk management (adopted from [10]).

The risk analysis of a system consists of the use of all available information to estimate the risk to individuals or populations, property or the environment, from identified hazards. The risk assessment further includes risk evaluation (acceptance or treatment) as 
indicated in Figure 10.1 (adopted from [10]). The whole procedure of risk assessment is typically an iterative process as indicated in Figure 10.2 (adopted from [7]). The first step in the risk analysis involves the context (scope) definition related to the system and the subsequent identification of hazards.

The system is understood [10] as a bounded group of interrelated, interdependent or interacting elements forming an entity that achieves in its environment a defined objective through the interaction of its parts. In the case of technological hazards related to civil engineering works, a system is normally formed from a physical subsystem, a human subsystem, their management, and the environment. Note that the risk analysis of civil engineering systems (similar to the analysis of most systems) usually involves several interdependent components (for example human life, injuries, and economic loss).

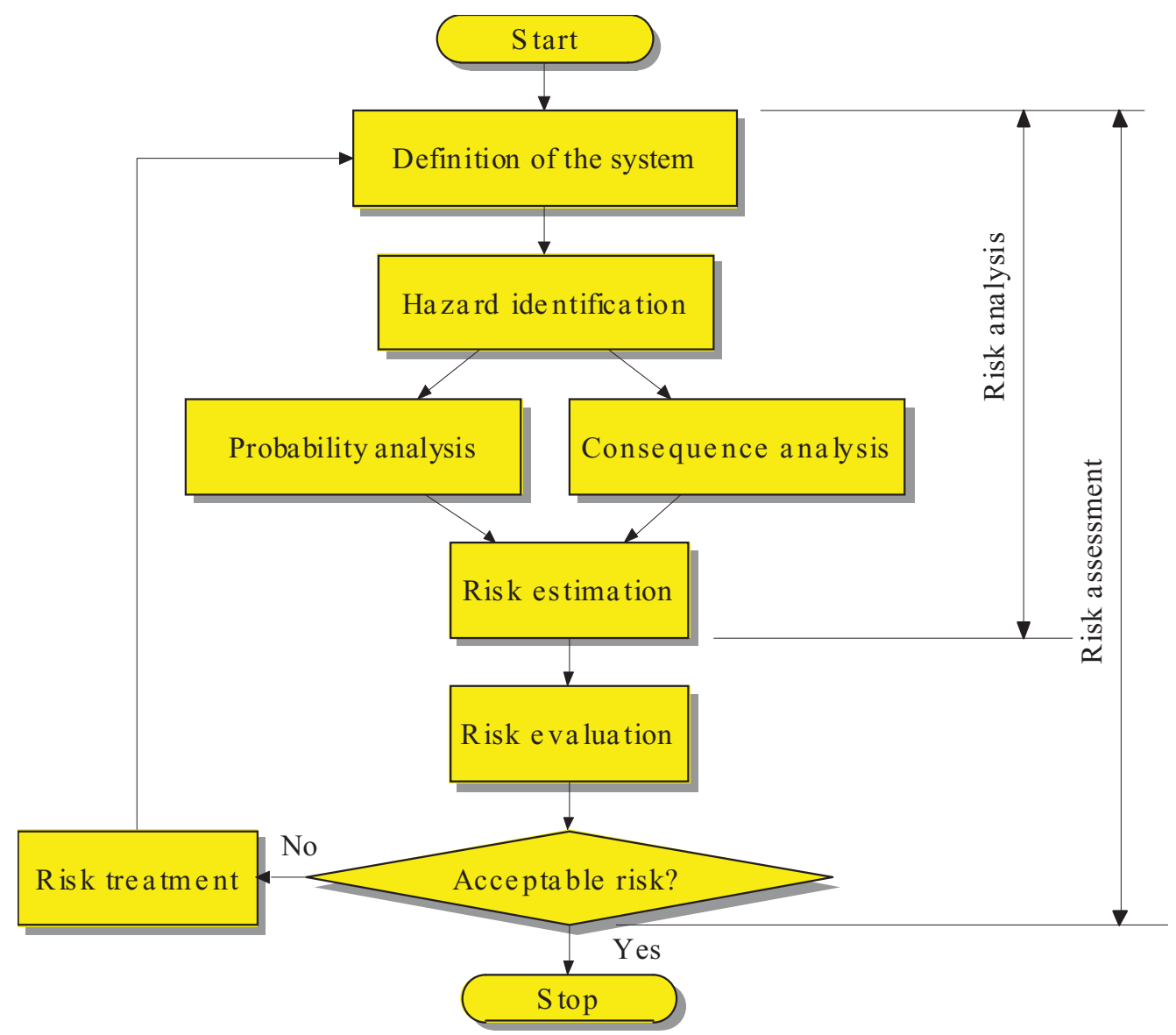

Figure 10.2. Flowchart of iterative procedure for risk assessment (adopted from [7]).

Any technical system may be exposed to a multitude of possible hazard situations. In the case of civil engineering facilities, hazard situations may include both environmental effects (wind, temperature, snow, avalanches, rock falls, ground effects, water and ground water, chemical or physical attacks, etc.) and human activities (usage, chemical or physical attacks, fire, explosion, etc.). As a rule, hazard situations due to human errors are more significant than hazards due to environmental effects. 


\subsection{Hazard identification}

A hazard is a set of circumstances, possibly occurring within a given system, with the potential for causing events with undesirable consequences. For instance the hazard of a civil engineering system may be a set of circumstances with the potential to cause an abnormal action (for example fire, explosion) or environmental influence (flooding, tornado) and/or insufficient strength or resistance or an excessive deviation from intended dimensions. In the case of a chemical substance, the hazard may be a set of circumstances likely to cause its exposure [10].

Hazard identification and modelling is the process of recognising the hazard and defining its characteristics in time and space. In the case of civil engineering systems the hazards $H_{i}$ may be linked to various design situations of the building (as defined in [7]) including persistent, transient and accidental design situation. As a rule, $H_{i}$ are mutually exclusive situations (for example persistent and accidental design situations of a building). Then if the situation $H_{i}$ occurs with the probability $\mathrm{P}\left\{H_{i}\right\}$, it holds $\sum \mathrm{P}\left\{H_{i}\right\}=1$. If the situations $H_{i}$ are not mutually exclusive, then the analysis becomes more complicated.

Note that in some documents (for example in the recent European document EN 1990 [1]) the hazard is defined as an event, while in risk analysis [10] it is usually considered a condition with the potential for causing events, thus as a synonym for danger.

\subsection{Definition and modelling of relevant scenarios}

A hazard scenario is a sequence of possible events for a given hazard leading to undesired consequences. Identifying what might go wrong with the system or its subsystem is a crucial task for risk analysis. It requires detailed examination and understanding of the system [10]. Nevertheless, a given system is often part of a larger system. Consequently, modelling and subsequent analysis of the system is a conditional analysis.

The modelling of relevant scenarios may be dependent on specific characteristics of the system. For this reason a variety of techniques have been developed for the identification of hazards (for example PHA HAZOP) and for the modelling of relevant scenarios (fault tree, event tree/decision trees, causal networks). A detailed description of these techniques is beyond the scope of this text but may, however, be found in $[6,7,8,9]$ and other literature.

\subsection{Estimation of probabilities}

Probability is generally the likelihood or degree of certainty of a particular event occurring during a specified period of time. In particular, the reliability of a structure is often expressed as the probability related to a specific requirement and a given period of time, for example 50 years $[1,2]$.

Assuming that a system may be found in mutually exclusive situations $H_{i}$, and the failure $F$ of the system (for example of the structure or its element) given a particular situation $H_{i}$ occurs with the conditional probability $\mathrm{P}\left\{F \mid H_{i}\right\}$, then the total probability of failure $p_{F}$ is given by the law of total probability (see for example [11]) as:

$$
p_{F}=\sum_{i} \mathrm{P}\left\{H_{i}\right\} \mathrm{P}\left\{F \mid H_{i}\right\}
$$

Equation (10.1) can be used for the modification of the partial probabilities $\mathrm{P}\left\{H_{i}\right\} \mathrm{P}\left\{F \mid H_{i}\right\}$ (appropriate to the situations $H_{i}$ ) with the aim to comply with the design condition $p_{F}<p_{\mathrm{t}}$, where $p_{\mathrm{t}}$ is a specified target probability of failure. The target value $p_{\mathrm{t}}$ may 
be determined using the probabilistic optimisation of an objective function describing, for example, the total cost.

The conditional probabilities $\mathrm{P}\left\{F \mid H_{i}\right\}$ must be determined by a detailed probabilistic analysis of the respective situations $H_{i}$ under relevant scenarios. The traditional reliability methods [2] assume that the failure $F$ of the system can be well defined in the domain of the vector of basic variables $\boldsymbol{X}$. For example, it is assumed that a system failure may be defined by the inequality $\mathrm{g}(\boldsymbol{x})<0$, where $\mathrm{g}(\boldsymbol{x})$ is the so-called limit state function, where $\boldsymbol{x}$ is a realisation of the vector $\boldsymbol{X}$. Note that $\mathrm{g}(\boldsymbol{x})=0$ describes the boundary of the limit state, and the inequality $\mathrm{g}(\boldsymbol{x})>0$ the safe state of a structure.

If the joint probability density $\mathrm{f}_{\boldsymbol{X}}\left(\boldsymbol{x} \mid H_{i}\right)$ of basic variables $\boldsymbol{X}$ given the situation $H_{i}$ is known, the conditional probability of failure $\mathrm{P}\left\{F \mid H_{i}\right\}$ can then be determined using the integral

$$
\mathrm{P}\left\{F \mid H_{i}\right\}=\int_{\mathrm{g}(\boldsymbol{x})<0} \mathrm{f}_{X}\left(\boldsymbol{x} \mid H_{i}\right) \mathrm{d} \boldsymbol{x}
$$

It should be mentioned that the probability $\mathrm{P}\left\{F \mid H_{i}\right\}$, calculated using equation (10.2), suffers generally from two essential deficiencies:

- uncertainty in the definition of the limit state function $\mathrm{g}(\boldsymbol{x})$,

- uncertainty in the theoretical model for the density function $\mathrm{f}_{\boldsymbol{X}}\left(\boldsymbol{x} \mid H_{i}\right)$ of basic variables $\boldsymbol{X}$.

These deficiencies are most likely the causes of the observed discrepancy between the determined probability $p_{F}$ and actual frequency of failures; this problem is particularly disturbing in the case of fire. Yet, the probability requirement $p_{F}<p_{\mathrm{t}}$ is generally accepted as a basic criterion for the design of structures.

In a risk analysis we need to know not only the probability of the structural failure $F$, but probabilities of all events having unfavourable consequences. In general, the situations $H_{i}$ may cause a number of events $E_{i j}$ (for example excessive deformations, full development of the fire). The required conditional probabilities $\mathrm{P}\left\{E_{i j} \mid H_{i}\right\}$ must be estimated by a separate analysis using various methods, for example the fault tree method or causal networks.

\subsection{Estimation of consequences}

Consequences are possible outcomes of a desired or undesired event that may be expressed verbally or numerically to define the extent of human fatalities and injuries or environmental damage and economic loss [7]. A systematic procedure to describe and/or calculate consequences is called the consequence analysis. Obviously, consequences are generally not one-dimensional. However, in specific cases they may be simplified and described by several components only, for example by human fatalities, environmental damage and costs. At present only various costs have usually been included. It is assumed that adverse consequences of the events $E_{i j}$ can normally be expressed by several components $C_{i j, k}$, where the subscript $k$ denotes the individual components (for example the number of lost lives, the number of human injuries and the damage expressed in a certain currency).

\subsection{Estimation of risk}

Risk is a measure of the danger that undesired events represent for humans, the environment or economic values. It is commonly expressed in the probability and consequences of the undesired events. It is often estimated by the mathematical expectation of 
the consequences of an undesired event. Then it is the product "probability $\times$ consequences". However, a more general interpretation of risk involves probability and consequences in a non-product form. This presentation is sometimes useful, particularly when a spectrum of consequences, with each magnitude having its own probability of occurrence, is considered [9].

The estimation of risk is a process used to produce an estimate of the measure of risk. As already stated above, the risk estimation is based on the hazard identification and generally contains the following steps: scope definition, frequency analysis, consequence analysis, and their integration [10]. If there is one-to-one mapping between the consequences (utility) $C_{i j, k}$ and the events $E_{i j}$, then the total risk $R_{k}$ related to the considered situations $H_{i}$ is the sum

$$
R_{k}=\sum_{i j} C_{i j, k} \mathrm{P}\left\{E_{i j} \mid H_{i}\right\} \mathrm{P}\left\{H_{i}\right\}
$$

If the dependence of consequences on events is more complicated than just one-to-one mapping, then equation (10.3) will have to be modified. A practical example of equation (10.3) can be found in [9], where an attempt to estimate the risk due to persistent and fire design situation is presented.

In some cases it is possible to deal with one-component risk $R$ only. Then the subscript $k$ in equation (10.3) may be omitted. Moreover, the probability of undesired events may depend on the vector of basic variables $\boldsymbol{X}$. Then the total risk $R$ may be formally written as

$$
R=\int C(\boldsymbol{x}) \mathrm{f}_{\boldsymbol{X}}(\boldsymbol{x}) \mathrm{d} \boldsymbol{x}
$$

where $R(\boldsymbol{x})$ denotes the degree of risk as a function of basic variables $\boldsymbol{X}$, and $\mathrm{f}_{\boldsymbol{X}}(\boldsymbol{x})$ denotes the joint probability density function $\boldsymbol{X}$.

\subsection{Logic trees}

A number of different logic (decision) trees (fault tree, event tree, cause/consequence chart) have been developed to analyse the risk of a system ([19] to [23]). Applications of logic trees significantly improve the completeness and clarity of an engineering work. The use of this kind of tool is widespread in risk analysis and implies some important advantages. Influences of the environment and of human activities can easily be considered simultaneously. Logic trees can also enable the detection of the most effective countermeasures. Furthermore, they can be easily understood by inexperienced persons and therefore can provide very effective communication means between experts and public authorities.

The fault tree can be defined as a logical diagram for the representation of the combinations of influences that can lead to an undesired event. When establishing a fault tree, the undesired event constitutes the starting point. Going out from this event, possible causes are to be identified. The possible causes and consequences are to be linked in a logic way, without introducing any loops. Every event that is not a consequence of the previous event has to be considered as an independent variable.

An example of a fault tree shown in Figure 10.3 describes the failure of a plane frame (indicated at the bottom of Figure 10.3).

Fault trees can be used to clarify the causes of failures in a case where they are unknown. The most common application, however, consists in detecting possible causes of undesirable events before they can occur. Since the fault trees also show the possible consequences of events, they are very useful for the establishment of the most accurate measures for prevention of these events. 


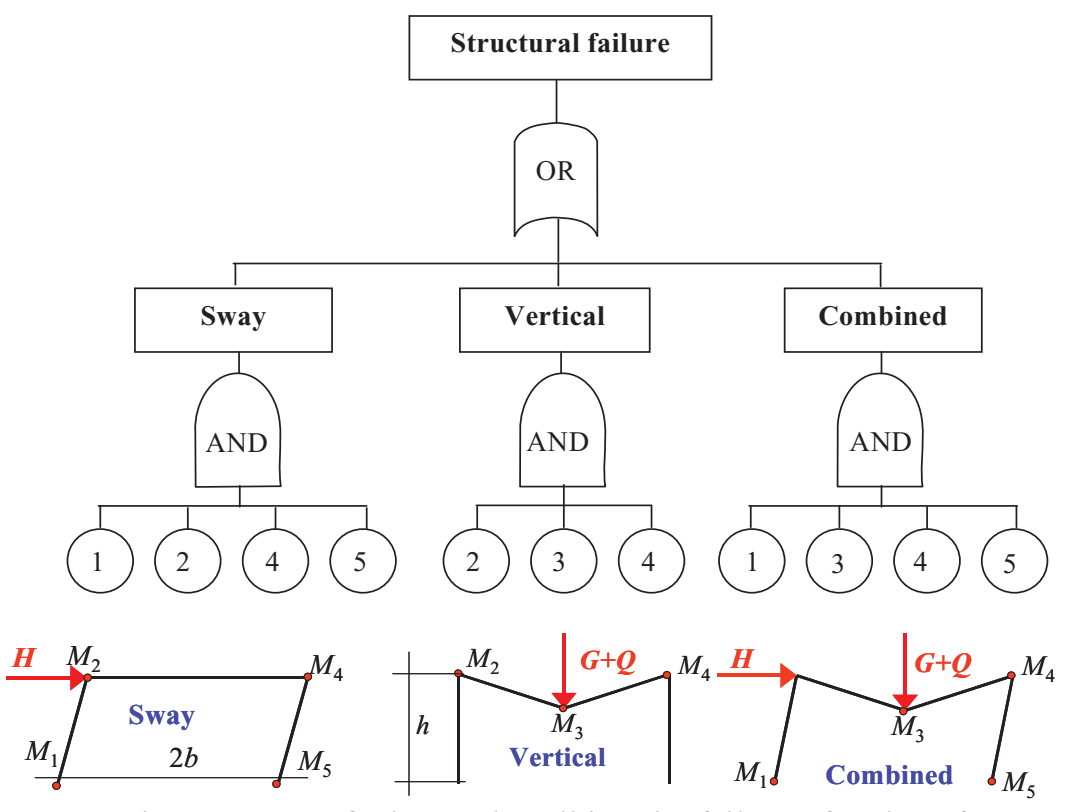

Figure 10.3. A fault tree describing the failure of a plane frame.

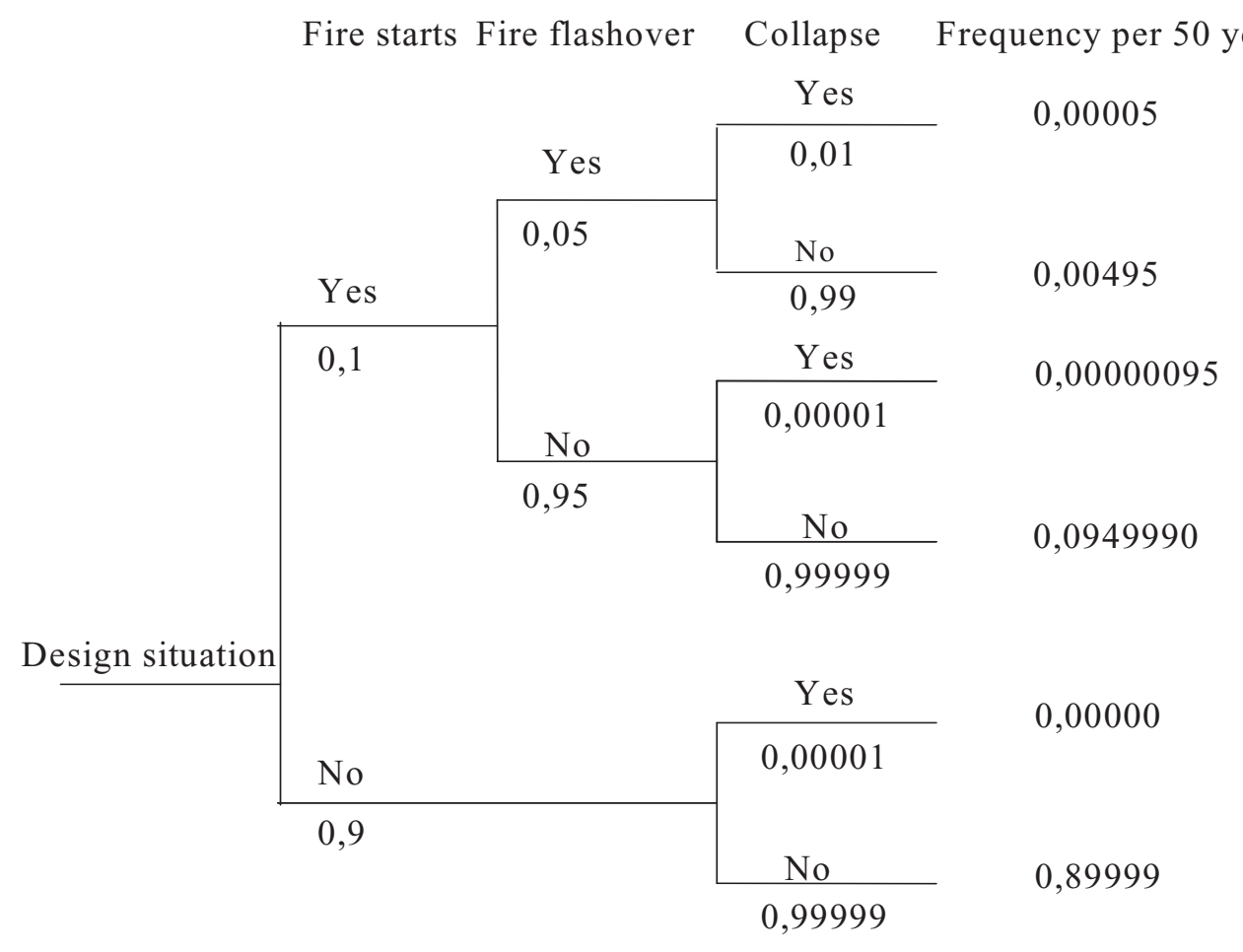

Figure 10.4. An event tree describing the collapse of a structure under persistent and fire design situations (all data are related to 50-year period of an administrative building having the fire compartment area $250 \mathrm{~m}^{2}$ without sprinklers and with a protected structure). 
An event tree identifies possible subsequent events (see Figure 10.4) starting from an initial event. Each path consists of a sequence of events and ends up at the consequence level (for example at structural failure, see Figure 10.4). The aim of the event tree analysis is to identify possible consequences of an initial event and to calculate the probability of the occurrence of these consequences corresponding to a different sequence of events.

Logic trees may be supplemented by the consequences of events; the graphic representation of such a tree is called the cause/consequence-chart. The consequence chart corresponds to an event tree with a suitable representation of expected consequences. For example Figure 10.4 may include consequences linked to each failure probability (frequency per year) of the structural collapse under given conditions. Then the tree may be used for the cause/consequence or risk (utility) analysis.

The simplest form of the cause/consequence consideration is the so-called prioranalysis of the risk (utility), when the basic statistical and probabilistic information is available prior to any decision or activity. The prior analysis is an assessment of the risk associated with different decisions; it is commonly used for comparing the risks corresponding to different decisions. The posterior decision analysis differs from the prior analysis by considering possible changes in the branching probabilities and/or the consequences due to risk reducing measures, risk mitigating measures, and the collection of additional information. The posterior decision analysis may be used to evaluate different additional activities affecting the total risk.

Another important modification of logic trees is known as the pre-posterior decision analysis. The aim of the pre-posterior decision analysis is to identify the optimal decisions with regard to activities that may be performed in the future, for example a planning of risk reducing activities and/or the collection of new information. An important pre-requisite for the pre-posterior decision analysis is the consideration of future actions that may be applied taking into account the results of the planned activities.

\subsection{Bayesian network}

Another promising tool for risk analysis seems to be the Bayesian (belief) causal networks $[19,26]$. A simple example of the causal network is shown in Figure 10.5. The network containing only four chance nodes describes the structural failure under persistent and fire design situations similar to the event tree in Figure 10.4. Compared with the event tree shown in Figure 10.4 the network in Figure 10.5 also includes the effect of sprinklers (node B). Note that the directional arrows in Figure 10.5 indicate the causal links between interconnected chance nodes.

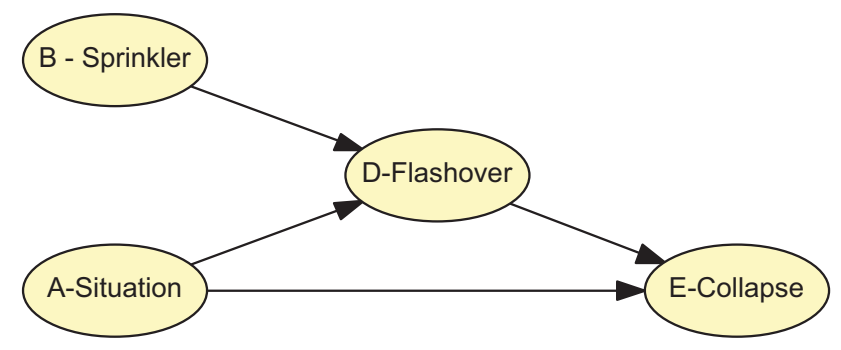

Figure 10.5. The causal network describing the structural failure under persistent and fire design situations. 
The collapse of a structure depends on the probability of persistent and fire situations and on the conditional probabilities of the full development of fire, which depend on the capability of sprinklers and on the conditional probability of the structural collapse under the conditions given by parent nodes (for example when fire is fully developed - fire flashover). Obviously the causal network representation seems to be much more effective than the event tree version. Moreover, each node may have several states. Consequently, the input data are not indicated directly in the graphic representation of the network but are given in the tables of conditional probabilities.

The basic principle of the probability calculation used in the Bayesian networks may be illustrated considering the nodes A, B and D of the network in Figure 10.5. One child node $D$ (Fire flashover) is dependent on two parent nodes: $A$ (Design situation) and $B$ (Sprinklers). If the parent nodes $A$ and $B$ have the discrete states $A_{i}$ and $B_{j}$, then the probability of the event $D_{k}$ (a particular state of the node $D$ ) is given by the formula

$$
\mathrm{P}\left(D_{k}\right)=\sum \mathrm{P}\left(D_{k} \mid A_{i} B_{j}\right) \mathrm{P}\left(A_{i}\right) \mathrm{P}\left(B_{j}\right)
$$

Equation (10.5) represents a fundamental theoretical tool for analysing the Bayesian network. The input data consist of the probabilities $\mathrm{P}\left(A_{i}\right)$ and $\mathrm{P}\left(B_{j}\right)$, and the conditional probabilities $\mathrm{P}\left(D_{k} \mid A_{i} B_{j}\right)$. These extensive data are based on available statistical evidence, probabilistic analysis or expert assessment (judgement) and are transparently summarised in the tables of conditional probabilities.

Bayesian networks supplemented by decision and utility nodes called influence diagrams $[19,26]$ provide a powerful tool for risk estimation. In fact, the influence diagram is a generalisation of the cause/consequence-chart discussed above. The main features of this tool are illustrated by the example shown in Figure 10.6, which is an extension of the fundamental task indicated in Figure 10.5. Figure 10.6 shows a simplified influence diagram, which has been developed recently [37] for the risk analysis of buildings under persistent and fire design situations.

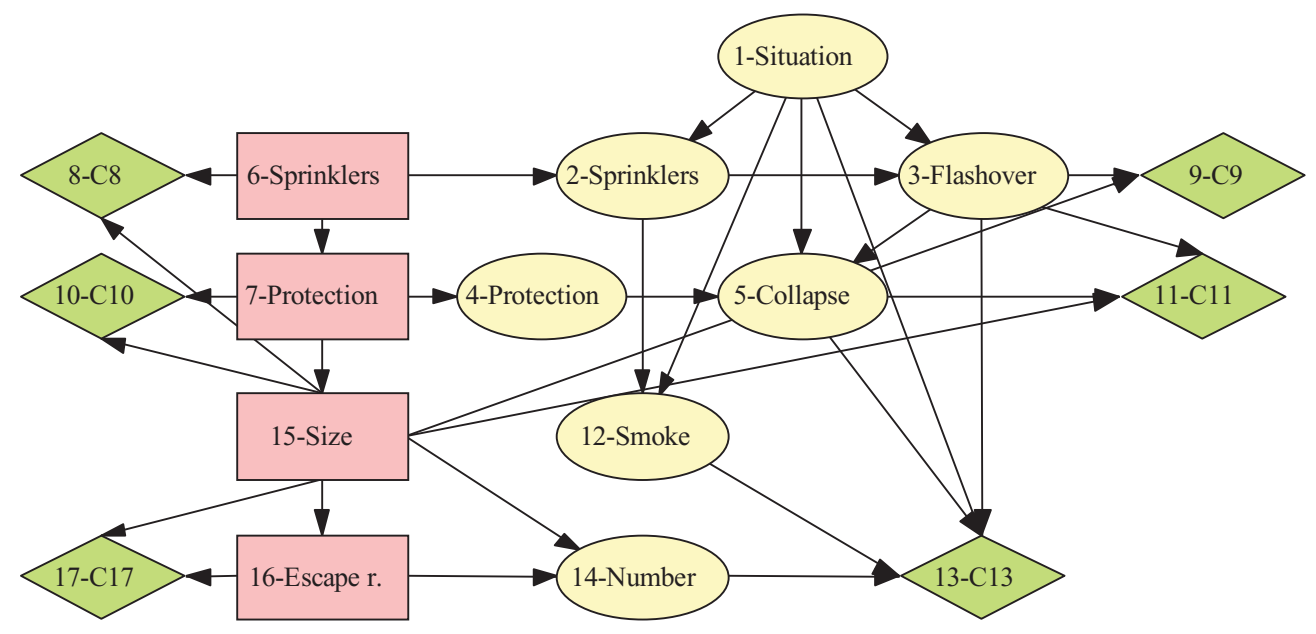

Figure 10.6. The Bayesian network describing a structure under normal and fire design situations. 
The network consists of seven chance nodes numbered 1, 2, 3, 4, 5, 12 and 14, four decision nodes $6,7,15$ and 16, and six utility nodes 10,9,10,11,13 and 17. The utility nodes represent the costs of various fire safety measures (nodes $8,10,17$ ), the damage to the building (nodes 9, 11), and injuries (node 13).

Directional arrows indicating the causal links between the parent and child nodes interconnect the chance, decision and utility nodes. All the causal links must be described by appropriate input data (conditional probabilities or utility units) linked to assumed states of the nodes. For example the utility nodes (except the utility node 13) are directly dependent on the size of the building (node 15). The utility node 13, describing the cost of injury, is affected by the size of the building through the number of endangered persons represented by the chance node 14. These data are often difficult to specify, and an expert assessment has to be made.

The chance nodes $1,2,3,4,5,12$ and 14 represent alternative random variables having two or more states. The node 1 -Situation describes the probability of fire start $p_{\mathrm{fi}, \mathrm{s}}=$ $\mathrm{P}\left(H_{2}\right)$ and the complementary probability $1-p_{\mathrm{fi}, \mathrm{s}}$ of the normal situation $H_{1}$. The chance node 2-Sprinklers describes the functioning of sprinklers provided that the decision (node 6) is positive; the probability of the active state of the sprinklers given the fire start is assumed to be very high, for example 0,999 . The chance node 3-Flashover has two states: the design situation $H_{3}$ (the fire design situation without flashover) and $H_{4}$ (the fire design situation with flashover when the fire is fully developed).

When sprinklers are installed, the flashover in a compartment of $250 \mathrm{~m}^{2}$ has the positive state with the conditional probability 0,002 ; if sprinklers are not installed, then $\mathrm{P}\left\{H_{4} \mid H_{2}\right\}=0,066$ [40]. It is assumed that with the probabilities equal to the squares of the above values the fire will flash over the whole building, thus the values 0,000004 and 0,0044 are considered for the chance node 3. The chance node 4-Protection (introduced for formal computational reasons) has identical states to the decision node 7-Protection. The chance node 5-Collapse represents the structural failure that is described by the probability distribution linked to three child nodes $(1,3,4)$. This situation can hardly be modelled using a decision tree. Note that the probability of collapse in the case of fire but not flashover may be smaller than in a persistent situation, due to a lower imposed load.

\subsection{Decision-making}

The decision-making is generally based on the process of risk acceptance and option analysis (see Figure 10.1) that is sometimes referred to as the risk evaluation. The risk acceptance is based on various criteria of risk that are the reference points against which the results of the risk analysis are to be assessed. The criteria are generally based on regulations, standards, experience, and/or theoretical knowledge used as a basis for the decision about the acceptable risk. Acceptance criteria and the criteria of risk may sometimes be distinguished [9]. Various aspects may be considered, including cultural, social, psychological, economic and other aspects [10], [21]. Generally, the acceptance criteria may be expressed verbally or numerically [38].

Assuming for example that the acceptance limits $C_{k, \mathrm{~d}}$ for the components $C_{k}$ are specified, then it is possible to design the structure on the basis of acceptable risks using the criterion $C_{k}<C_{k, \mathrm{~d}}$, which may supplement the probability requirement $p_{F}<p_{\mathrm{t}}$.

It should be noted that various levels of risk might be recognised, for example acceptable risk, tolerable risk, and objective risk [38] (see the definitions of these terms). It is a remarkable fact that the public seems to be generally better prepared to accept certain risks than to stand for specified probabilities of failure [30]. 


\subsection{Concluding remarks}

The risk is commonly estimated by the mathematical expectation of the consequences of an undesired event that often leads to the product "probability $\times$ consequences". As a rule, the risk of civil engineering systems is a multidimensional quantity having several components.

Risk analysis is based on hazard identification and generally contains the following steps: the scope definition, hazard identification, definition and modelling of hazard scenarios, estimation of probabilities, estimation of consequences, estimation of risk and decisionmaking.

The most important contribution of risk analysis and assessment consists of the systematic consideration of various consequences. Several techniques are available at present: the decision trees, the Bayesian belief networks and influence diagrams. Available experience indicates that the Bayesian belief networks provide a transparent, logical and effective tool for analysing engineering systems. It should, however, be underlined that any analysis of an engineering system is always dependent on the assumed input data, often of a very uncertain nature. The input data should be estimated with due regard to the specific technological and economic conditions of a given system. In particular, the economic, social and environmental consequences of adverse events should be further investigated.

It appears that the methods of risk analysis and assessment may significantly contribute to a further improvement of current engineering design. The remarkable fact that the public is better prepared to accept certain risks than to stand for specified probabilities of failure will make the application of risk assessment easier. It is therefore anticipated that in the near future probabilistic methods in engineering will be supplemented by criteria for acceptable risks. Obviously the proposed new International Standard ISO will be extremely useful. 


\section{APPENDIX: TERMINOLOGY OF RISK ASSESSMENT}

Considering the main area of their application the terms are subdivided into four groups: general terms, the terms related to risk communication, the terms related to risk assessment and the terms related to risk management and control. Mutual links between different terms are illustrated in Figure 10.1 indicating a framework for risk management and in Figure 10.2 showing a fundamental flowchart of the risk assessment procedure.

\section{GENERAL TERMS}

1.1 Hazard: An event or a combination of events with the potential for undesirable consequences.

Note 1: For instance an abnormal action or environmental influence and/or insufficient strength or resistance or an excessive deviation from intended dimensions, in the case of a chemical, the potential that the substance has for causing adverse effects at various levels of exposure. [10].

Note 2: In some documents (for example in the recent draft of EN 1990 [1]) the hazard is defined as an event, while in risk analysis [10] it is considered a condition with a potential for causing an event. Thus, in risk analysis the hazard is a synonym to danger.

1.2 Hazard scenario: A sequence of possible events related to a given hazard leading to undesired consequences.

Note: To identify what might go wrong with the system or its subsystem is crucial to a risk analysis. It requires the system to be examined and understood in considerable detail [38].

1.3 Event: Occurrence of a particular set of circumstances.

Note: An undesired event is an event, which can cause negative consequences like human fatalities and injuries or environmental damage and economic losses.

1.4 Probability: The likelihood or degree of belief of a particular event occurring within a specified reference (time, number of repetitions, etc.).

Note: The probability may depend significantly on the time period during which the particular event may occur.

1.5 Objective probability: The probability determined using theoretical arguments or adequate statistical data.

1.6 Subjective probability: The probability determined using intuition and relevant experience.

1.7 Consequence: The utility assigned to the event in accordance with the preferences of the decision maker.

Note 1: There can be more than one consequence from one event.

Note 2: Consequences can range from positive to negative.

Note 3: Consequences can be expressed qualitatively or quantitatively. 
1.8 Risk: The expected consequences associated with an activity. Risks may be related to adverse events for humans, qualities of the environment or economic values. In general the risk is the combination of probability of an event and its consequence [7]. Note 1: The risk is often estimated by the mathematical expectation of the consequences of an undesired event. Then it is the product "probability $x$ consequences". However, a more general interpretation of the risk involves probability and consequences in a non-product form. This presentation is sometimes useful, particularly when a spectrum of consequences, each having its own corresponding probability of occurrence, is considered [38].

Note 2: Various levels of risk may be recognised, for example acceptable risk, tolerable risk and objective risk [38] (see the definition of these terms).

1.9 Objective risk: An estimate of the system risk, obtained using theoretical arguments or adequate statistical data (for example the annual expected fatalities from car accidents) or from quantified risk analysis methods (QRA, PRA).

1.10 Reliability: The ability of a structure or structural element to fulfil the specified requirements during a given period of time (for example design life).

Note 1: The reliability is often expressed as a probability related to a specific requirement and a period of time $[1,2]$.

Note 2: In respect of ultimate limit states, the reliability is often referred to as safety; in respect of serviceability limit states, the reliability is often referred to as serviceability $[1,2]$.

1.11 Safety: The state of being protected against hurt or injury, freedom from danger or hazard.

Note: In structural reliability safety is often understood as the reliability with regard to the ultimate limit state (see the definition of Reliability).

1.12 System: A bounded group of interrelated, interdependent or interacting elements forming an entity that achieves a defined objective in its environment through the interaction of its parts.

Note 1: This definition implies that the system is identifiable, is made up of interacting elements or subsystems, all elements are identifiable, and the boundary of the system can be identified [10].

Note 2: In terms of technological hazards, a system is normally formed from a physical subsystem, a human subsystem, their management and environment [10].

2.1 Risk communication: The exchange or sharing of information about risk between the decision-maker and other stakeholders.

Note: The information can relate to the existence, nature, form, probability, severity, acceptability, treatment or other aspects of risk.

2.2 Stakeholder: Any individual, group or organisation that can affect, be affected by, or perceive itself to be affected by a risk [8].

Note 1: The decision-maker is also a stakeholder. 
Note 2: The term "stakeholder" includes but has a broader meaning than the interested party (which is defined in ISO 9000:2000).

2.3 Interested party: A person or group having an interest in the performance or success of an organisation [7].

Examples: Customers, owners, people in an organisation, suppliers, bankers, unions, partners or society.

Note: A group can comprise an organisation, a part thereof, or more than one organisation.

(ISO 9000: 2000, definition 3.3.7).

2.4 Risk perception: The way in which a stakeholder views a risk, based on a set of values or concerns [7].

Note 1: The risk perception depends on the stakeholders' needs, issues, knowledge and preferences.

Note 2: The risk perception can be significantly subjective.

2.5 Criteria of risk: The reference points against which the results of the risk analysis are to be assessed. The criteria are generally based on regulations, standards, experience, and/or theoretical knowledge used as a basis of the decision on acceptable risk.

Note: Various aspects may be considered, including cultural, social, psychological, economic and other aspects [38]. The acceptance criteria may be expressed verbally or numerically [38].

2.6 Acceptable risk: A level of risk, which is generally not seriously perceived by society, and which may be considered as a reference point in criteria of risk.

Note: It is expected that various aspects including cultural, social, psychological, economic and other aspects will influence the risk perception in society (see also the definition of risk criteria).

\section{TERMS RELATED TO RISK ASSESSMENT}

3.1 Hazard identification: A process of recognising the hazard and defining its characteristics.

3.2 Causal analysis: A systematic procedure for describing and/or calculating the probability of causes for desired or undesired events.

3.3 Consequence analysis: A systematic procedure to describe and/or calculate consequences.

3.4 Risk analysis: The use of available information concerning relevant hazard situations for estimating the risk for individuals or populations, property or environment.

Note: The risk analysis generally involves the context (scope) definition, hazard identification, and risk estimation [10].

3.5 Risk assessment: A process of risk analysis, risk acceptance and option analysis. 
Note: In some documents [1] the risk assessment is defined as risk analysis and risk evaluation, where the risk evaluation covers risk acceptance and option analysis (see the definition of risk evaluation).

3.6 Risk estimation: A process used to produce the estimate of the risk measure.

Note: The risk estimation is based on hazard identification and generally contains the following steps: scope definition, probability analysis, consequence analysis, and their integration [10].

3.7 Risk evaluation: A process of risk acceptance and option analysis.

3.8 Sensitivity analysis: A systematic procedure to describe and/or calculate the effect of variations in the input data and underlying assumptions in general on the final result.

3.9 Option analysis: A process used to identify a range of possible alternatives for managing the risk.

TERMS RELATED TO RISK MANAGEMENT AND CONTROL

4.1 Risk management: The complete process of risk assessment and risk control.

Note: The entire risk management is schematically indicated in Figure 10.1 (adopted from [10]).

4.2 Risk treatment: A process of selection and implementation of measures to modify risk [9].

Note 1: The term "risk treatment" is sometimes used for the measures themselves. Note 2: The risk treatment measures can include avoiding, optimising, transferring or retaining risk.

4.3 Safety management: A systematic process undertaken by an organisation in order to attain and maintain a level of safety that complies with the defined objectives.

4.4 Tolerable risk: A level of risk which an individual or society is willing to accept to secure certain benefits, assuming that the risk will be properly controlled.

Note: The tolerable risk may not be negligible, but it should be kept under review and permanent control.

4.5 Risk control: Actions implementing risk management decisions.

Note: The risk control may involve monitoring, reevaluation, and compliance with decisions.

4.6 Risk optimisation: A process, related to a risk, to minimise the negative and to maximise the positive consequences and their respective probabilities [8].

Note 1: In the context of safety, the risk optimisation is focused on reducing the risk. Note 2: The risk optimisation depends upon risk criteria, including costs and legal requirements.

Note 3: A risk associated with risk control can be considered. 
4.7 Risk reduction: Actions taken to lessen the probability, negative consequences, or both, associated with a risk [7].

4.8 Mitigation: Limitation of any negative consequence of a particular event [8].

4.9 Risk avoidance: The decision not to become involved in, or action to withdraw from, a risk situation.

Note: The decision may be taken based on the result of risk evaluation.

4.10 Risk transfer: Sharing with another party the burden of loss or the benefit of gain, for a risk [7].

Note 1: Legal or statutory requirements can limit, prohibit or mandate the transfer of a certain risk.

Note 2: The risk transfer can be carried out through insurance or other agreements.

Note 3: The risk transfer can create new risks or modify existing risk.

Note 4: Relocation of the source is not the risk transfer.

4.11 Risk financing: Provision of funds to meet the cost of implementing risk treatment and related costs [7].

Note: In some industries, the risk financing refers to funding the financial consequences related to the risk only.

4.12 Risk retention: Acceptance of the burden of loss, or the benefit of gain, from a particular risk [7].

Note 1: The risk retention includes the acceptance of risks that have not been identified.

Note 2: The risk retention does not include treatments involving insurance, or transfer by other means.

Note 3: There can be variability in the degree of acceptance and dependence on risk criteria.

4.13 Risk acceptance: The decision to accept a risk.

Note 1: The verb "to accept" is chosen to convey the idea that acceptance has its basic dictionary meaning.

Note 2: The risk acceptance depends on risk criteria.

4.14 Residual risk: A risk remaining after risk treatment [8].

Note: See also ISO/IEC Guide 51 [7] for safety aspects. 


\section{REFERENCES}

\section{Standards}

[1] EN 1990 Eurocode - Basis of structural design. CEN, 2002.

[2] ISO 2394, General principles on reliability for structures. 1998.

[3] ISO 13822 Assessment of existing structures. 2002.

[4] ISO 12491 Statistical methods for quality control of building materials and components. 1997.

[5] ISO 3534-1 Statistics - Vocabulary and Symbols - Part 1: Probability and general statistical terms. 1993.

[6] ISO 3534-2 Statistics - Vocabulary and Symbols - Part 2: Statistical quality control. 1993.

[7] ISO/IEC, Guide 51: Safety aspects - Guidelines for their inclusion in standards, 1999.

[8] ISO/IEC, Guide 73: Risk management - Vocabulary - Guidelines for use in standards, 2002.

[9] NS 5814, Requirements for risk analysis. 1991.

[10] CAN/CSA - Q634-91 Risk analysis requirements and guidelines.1991.

\section{Books}

[11] Ang A.H.-S. and Tang W.H., Probabilistic concepts in engineering planning and design. John Wiley and Sons, New York, 1975.

[12] Benjamin J.R. and Cornell C.A., Probability concept and decision for civil engineers. McGraw Hill, New York, 1970.

[13] CIRIA Report 63, Rationalization of safety and serviceability factors in structural codes. London, 1975.

[14] Devore J. and Farnum N., Applied Statistics for Engineers and Scientists. Thomson, London, 2005.

[15] Diamantidis D., Probabilistic Assessment of Existing Structures. Joint Committee on Structural Safety, RILEM, 2001.

[16] Gulvanessian H., Calgaro, J.-A. and Holický, M., Designer's Guide to EN 1990, Eurocode: Basis of Structural Design. Thomas Telford, London, 2002, 192 pp.

[17] Christensen P.T. and Baker M., Structural Reliability Theory and its Applications. Springer-Verlag, Berlin, 1982.

[18] Stewart Mark G. and Melchers Robert E., Probabilistic Risk Assessment of Engineering Systems. Chapman \& Hall, London, 1997.

[19] Jensen Finn V., Introduction to Bayesian networks. Aalborg University, Denmark, 1996.

[20] Madsen H.O., Krenk S. and Lind N.C., Methods of Structural Safety. PrenticeHall Inc., Englewood Cliffs, 1986.

[21] Melchers R.E., Structural Reliability: Analysis and Prediction. John Wiley \& Sons, New York, 1999.

[22] Nowak A.S. and Collins K.R., Reliability of Structures. McGraw Hill, 2000.

[23] Schneider J., Introduction to Safety and Reliability of Structures. IABSE, Zürich 1997. 


\section{Software products}

[24] STRUREL, Structural Reliability System (zahrnuje programy STATREL, COMREL, SYSREL a NASREL - COMREL version $8.00 \mathrm{z}$ roku 2003). RCP Consulting software, Munich, Germany.

[25] VaP, Variable Processor 2.0. Petschacher Conculting, Feldkirchen, Austria, 2003.

[26] Hugin system: Version 5.7, professional. Hugin Expert A/S, Niels Jernes Vej 10, DK-9220 Aalborg, Denmark, 2001. (see also software product GeNie, http//:genie.sis.pitt.edu).

\section{Publications on web sides}

[27] Probabilistic Model Code, Parts 1 to 4, Basis of design, Load and resistance models, Examples, JCSS, 2001-2002.

\section{Papers and conference contributions}

[28] Holický, M., Schneider, J., 2001: Structural Design and Reliability Benchmark Study. In: Safety, Risk and Reliability - Trends in Engineering; Zürich. IABSE, 2001; ISBN: 3-85748-102-4, pp. 929-938.

[29] Holický M. , Vorlíček M., Distribution Asymmetry in Structural Reliability. Acta Polytechnica, Vol. 35, No. 3/ 1995, pp. 75-85.

[30] Lewis R., The Public Perception of Risk, RSA Journal, 11/1995, pp. 52-63.

[31] Holický M., Vrouwenvelder T., Reliability Analysis of a Reinforced Concrete Column. Acta Polytechnica, Vol. 36, No. 2/1996, pp. 9.

[32] Holický M., Rackwitz R., Time-variant reliability of a column under variable loads with intermittencies. Proc. Safety and Reliability. A.A. Balkema, Rotterdam, 1998, pp. 977/984.

[33] Holický M., Risk Assessment in Advanced Engineering Design. Acta Polytechnica, Vol 43, No. 3/2003.

[34] Holický M., Risk assessment of steel buildings and occupants under fire situation. ICASP 9, Berkeley, 2003, pp. 163-168.

[35] Holický M., Mihashi H., Probabilistic Optimization of Concrete Cover Exposed to Carbonation. Proc. New Requirements for Materials and Structures. CTU, Prague 1998.

[36] Schneider J., Safety - A Matter of Risk, Cost and Consensus. Structural Engineering International. No. 4, November 2000, pp. 266-269.

[37] Holický M., Prospects for Advanced Engineering Design Based on Risk Assessment. Acta Polytechnica, Vol. 41, No. 4-5/2001; ISSN: 1210-2709; pp. $8-12$.

[38] CIB TG 32, Report 259 Risk assessment and risk communication in civil engineering, CIB Secretariat 2001.

[39] Holický M., Marková J., Reliability of Concrete Elements Designed for Alternative Load Combinations Provided in Eurocodes. Acta Polytechnica, 2003/1.

[40] Rackwitz, R., Computational techniques in stationary and non-stationary load combination - a review and some extensions. Technical Note, RCP-Consult, München, 1997. 


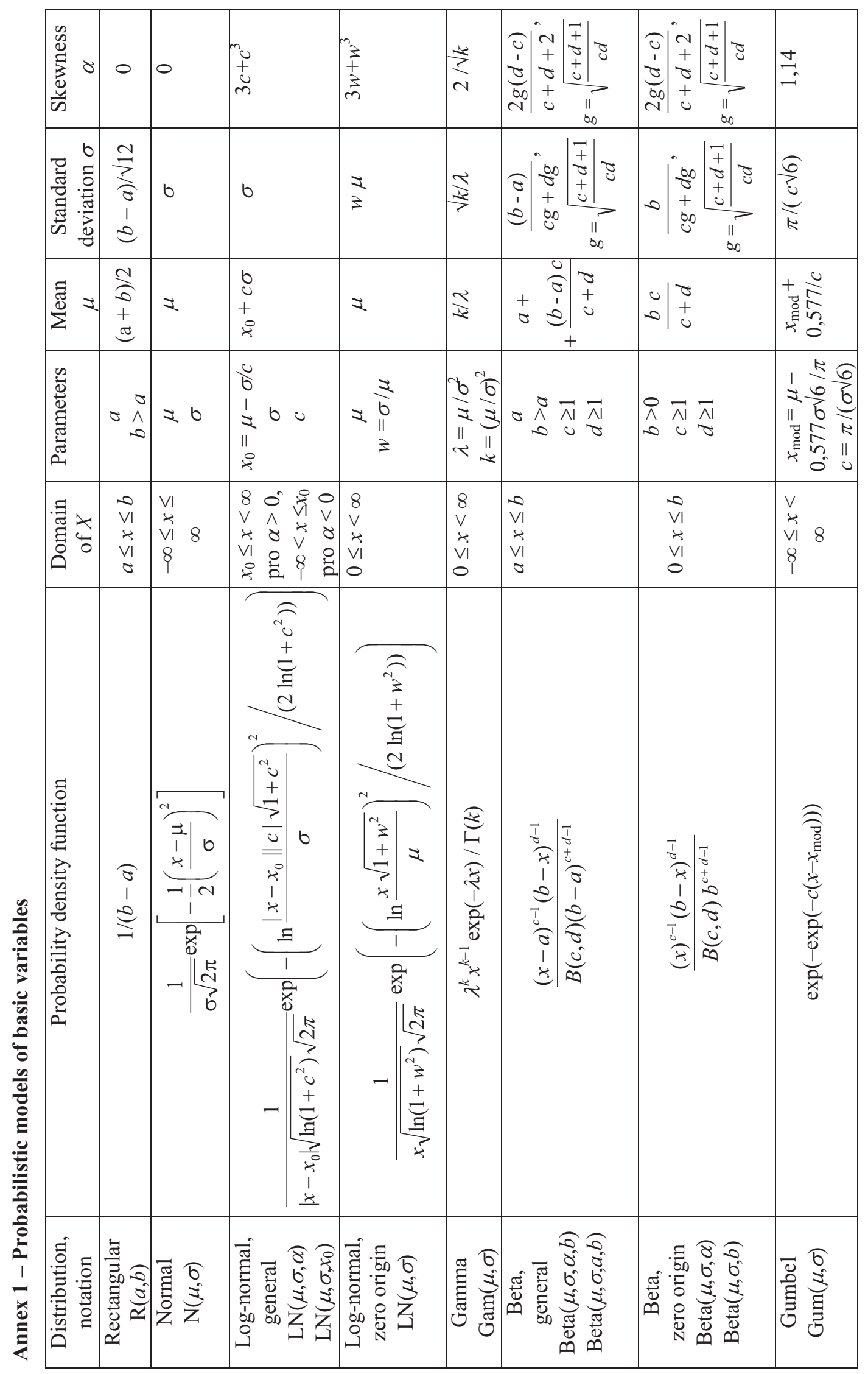




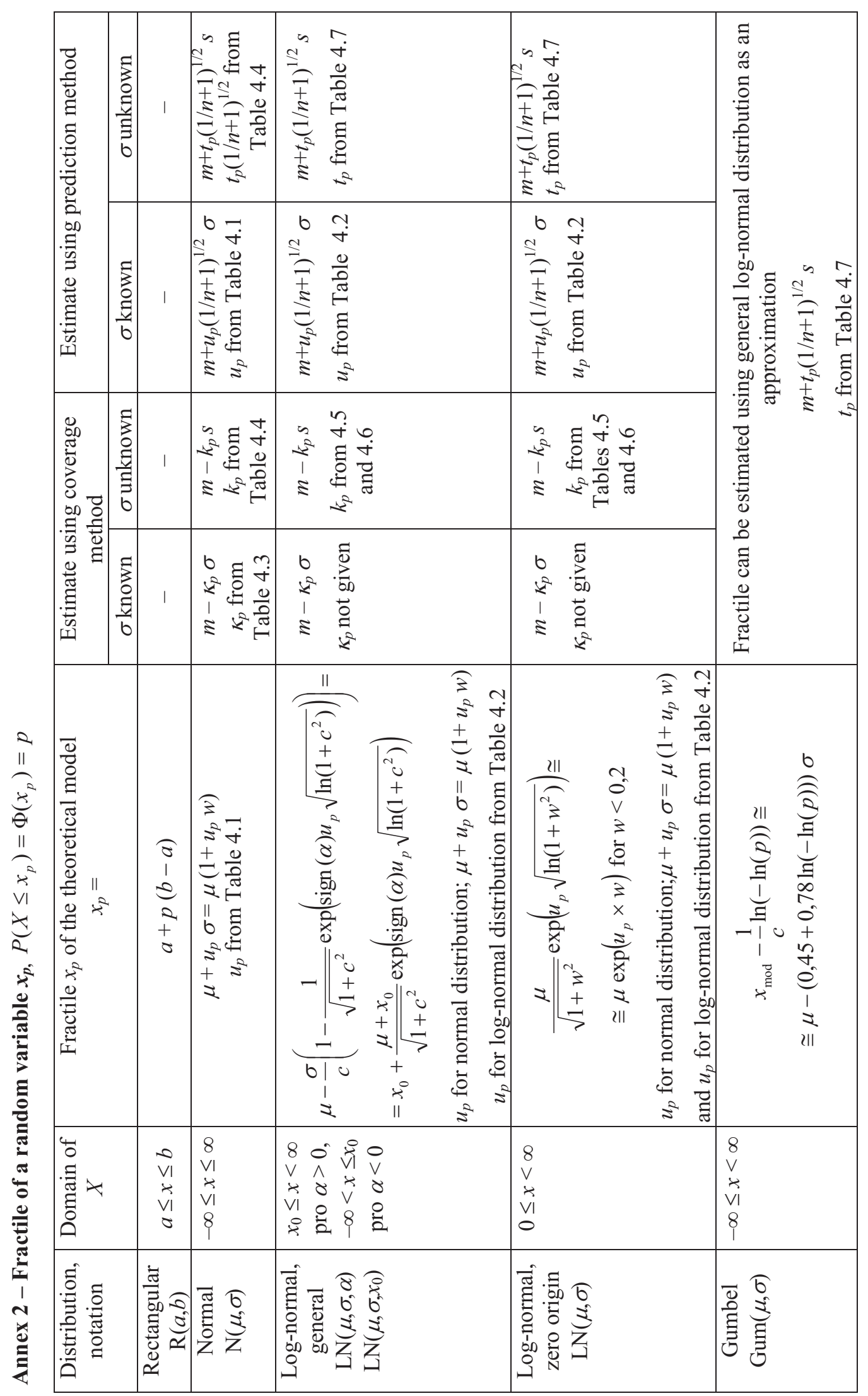




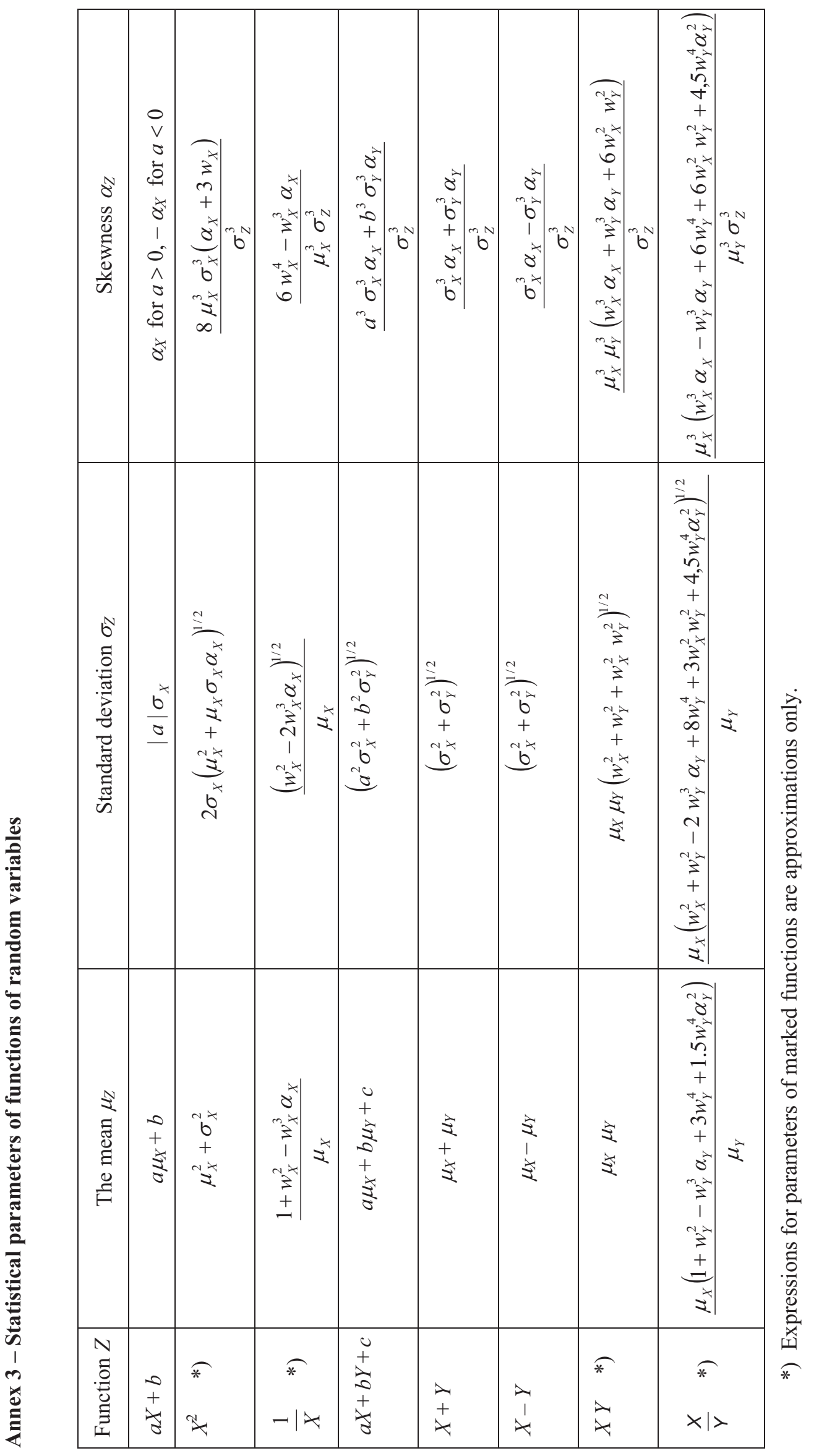




\section{Annex 4: Conventional probabilistic models of basic variables}

\section{Contents}

1 Introduction 162

2 Probabilistic models $\quad 162$

3 Comments on probabilistic models 164

3.1 Actions 164

3.2 Material property 169

$\begin{array}{ll}3.3 \text { Geometric data } & 169\end{array}$

3.4 Model uncertainties $\quad 170$

3.5 Time-variant parameters 171

4 Concluding remarks $\quad 173$

References 174

\section{Introduction}

Probabilistic models of basic variables used in different reliability studies often deviate one from the other. Obviously, the reliability studies based on different probabilistic models may lead to more or less different results and to undesirable discrepancies in recommendations concerning the partial safety factors, combination factors and other reliability elements. It is the aim of this Annex to propose conventional models in order to enable an efficient comparison of reliability studies of various structural members made of different materials (steel, concrete, composite). It is foreseen that this Annex may be used independently of the main text and that is why it is written as a self-contained document with its own references and figures.

Probabilistic models of basic variables presented in this study Annex are intended to be used primarily for calibration procedures expected in the near future in connection with implementation of Eurocodes [1, 2, 3, 4] and ISO standard [5] into the national systems of codes. Proposed models are specified considering middle values of action variances, common structural conditions and normal quality control of material properties. Recent documents of JCSS $[6,7]$, CIB reports $[8,9,10,11]$, SAKO report [13] and other references $[14,15,16]$ are taken into account.

\section{$2 \quad$ Probabilistic models}

The following conventional models of basic variables are primarily intended to be used in time-invariant reliability analyses (using Turkstra's combination rule) of simple reinforced concrete and steel members. However, the annual maximum value distribution supplemented by appropriate parameters describing time-variant properties can also be applied in time-variant reliability analysis.

Table 1 includes three fundamental categories of basic variables (actions, material strengths and geometric data) supplemented by uncertainty factors for action effects and structural resistance. Note that the data indicated in summary Table 1 represent only reasonable conventional models, which may not be adequate in some specific cases (for example for the wind load of high rise buildings). 
For the purpose of comparative and calibration studies the mean values $\mu_{X}$ of all the variables $X$ are related to the characteristic value $X_{\mathrm{k}}$ used in the design calculation. The last column of Table 1 shows the occurrence probability of value $X$ as smaller than the characteristic value $X_{\mathrm{k}}$

$$
\mathrm{P}\left\{X<X_{\mathrm{k}}\right\}=\Phi_{X}\left(X_{\mathrm{k}}\right)
$$

Here $\Phi_{\underline{X}}$ denotes the distribution function of the basic variable $X$. Note that due to several reasons (historical development of codified values, quality control of materials) these probabilities in general differ from those recommended for specifications of the characteristic values $X_{\mathrm{k}}$ in Eurocodes (for example the actual probability of the material strengths $X$ being less than $X_{\mathrm{k}}$ is only 0,02 instead of the recommended value 0,05 given in EN 1990 [1]).

Table 1. Conventional models of basic variables for time-invariant reliability analyses.

\begin{tabular}{|c|c|c|c|c|c|c|c|c|c|}
\hline No. & $\begin{array}{l}\text { Category } \\
\text { of variabl. }\end{array}$ & $\begin{array}{l}\text { Name of basic } \\
\text { variables }\end{array}$ & $\begin{array}{l}\text { Sym. } \\
X\end{array}$ & $\begin{array}{l}\text { Dimen } \\
\text { sion }\end{array}$ & $\begin{array}{l}\text { Dis- } \\
\text { trib. }\end{array}$ & $\begin{array}{l}\text { Mean } \\
\mu_{X}\end{array}$ & $\begin{array}{l}\text { St. dev. } \\
\sigma_{X}\end{array}$ & $\begin{array}{l}\text { Prob. } \\
\Phi_{X}\left(X_{\mathrm{k}}\right)\end{array}$ & $\begin{array}{l}\text { Refer- } \\
\text { ences }\end{array}$ \\
\hline 1 & Actions & Permanent $^{+}$ & $G$ & $\mathrm{kN} / \mathrm{m}^{2}$ & $\mathrm{~N}$ & $G_{\mathrm{k}}$ & $0,03-0,10 \mu_{X}$ & 0,5 & 6,8 \\
\hline 2 & & Imposed-5 years & $Q$ & $\mathrm{kN} / \mathrm{m}^{2}$ & $\mathrm{GU}$ & $0,2 Q_{\mathrm{k}}$ & $1,1 \mu_{X}$ & 0,995 & 6,9 \\
\hline 3 & & Imposed-50 y. ${ }^{++}$ & $Q$ & $\mathrm{kN} / \mathrm{m}^{2}$ & $\mathrm{GU}$ & $0,6 Q_{\mathrm{k}}$ & $0,35 \mu_{X}$ & 0,953 & 6,9 \\
\hline 4 & & Wind -1 year $*$ & $W$ & $\mathrm{kN} / \mathrm{m}^{2}$ & $\mathrm{GU}$ & $0,3 W_{\mathrm{k}}$ & $0,5 \mu_{X}$ & 0,999 & 6,10 \\
\hline 5 & & Wind -50 years $*$ & $W$ & $\mathrm{kN} / \mathrm{m}^{2}$ & GU & $0,7 W_{\mathrm{k}}$ & $0,35 \mu_{X}$ & 0,890 & 6,10 \\
\hline 6 & & Snow -1 year $* *$ & $S$ & $\mathrm{kN} / \mathrm{m}^{2}$ & $\mathrm{GU}$ & $0,35 S_{\mathrm{k}}$ & $0,70 \mu_{X}$ & 0,998 & 6,11 \\
\hline 7 & & $\begin{array}{l}\text { Snow } \\
-50 \text { year** }\end{array}$ & $S$ & $\mathrm{kN} / \mathrm{m}^{2}$ & GU & $1,1 S_{\mathrm{k}}$ & $0,30 \mu_{X}$ & 0,437 & 6,11 \\
\hline 8 & Material & Steel yield point & $\overline{f_{\mathrm{y}}}$ & $\mathrm{MPa}$ & $\mathrm{LN}$ & $f_{\mathrm{yk}}+2 \sigma$ & $0,07-0,10 \mu_{X}$ & 0,02 & $6,13-16$ \\
\hline 9 & & Steel strength & $f_{\mathrm{u}}$ & $\mathrm{MPa}$ & LN & $\kappa \mu_{f y} * * ;$ & $* 0,05 \mu_{X}$ & - & $6,13-16$ \\
\hline 10 & Strengths & Concrete & $f_{\mathrm{c}}$ & $\mathrm{MPa}$ & LN & $f_{\mathrm{ck}}+2 \sigma$ & $0,10-0,18 \mu_{X}$ & 0,02 & $6,13-16$ \\
\hline 11 & & Reinforcement & $f_{\mathrm{y}}$ & $\mathrm{MPa}$ & LN & $f_{\mathrm{yk}}+2 \sigma$ & $30 \mathrm{MPa}$ & 0,02 & $6,13-16$ \\
\hline 12 & Geometry & IPE profiles & $A, W, I$ & $\mathrm{~m}^{2,3,4}$ & $\mathrm{~N}$ & $0,99 X_{\text {nom }}$ & $0,01-0,04 \mu_{X}$ & $\cong 0,73$ & 6,16 \\
\hline 13 & steel sect. & L-section, rods & $A, W, I$ & $\mathrm{~m}^{2,3,4}$ & $\mathrm{~N}$ & $1,02 X_{\text {non }}$ & $0,01-0,02 \mu_{X}$ & $x \cong 0,16$ & 6,16 \\
\hline 14 & Geometry & Cross-section & $b, h$ & $\mathrm{~m}$ & $\mathrm{~N}$ & $b_{\mathrm{k}}, h_{\mathrm{k}}$ & $0,005-0,01$ & 0,5 & 6 \\
\hline 15 & concrete & Cover of reinf. & $a$ & $\mathrm{~m}$ & BET & $a_{\mathrm{k}}$ & $0,005-0,015$ & 0,5 & 6 \\
\hline 16 & cross-sect. & Additional ecc. & $e$ & $\mathrm{~m}$ & $\mathrm{~N}$ & 0 & $0,003-0,01$ & 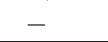 & 6 \\
\hline 17 & Model un- & Load effect factor & $\theta_{E}$ & - & $\mathrm{N}$ & 1 & $0,05-0.10$ & - & 6,7 \\
\hline 18 & certainties & Resistance factor $^{+}$ & $\theta_{R}$ & - & $\mathrm{N}$ & $1-1,25$ & $0,05-0,20$ & - & 6,7 \\
\hline
\end{tabular}

Notes: $++$

See also Table $1 \mathrm{a}$.

See also Table $1 b$.

See also Table $1 \mathrm{c}$.

See also Table 1d.

The coefficient $\kappa$ can be used to estimate the mean of $f_{\mathrm{u}}$ and can be considered as follows [6]:

$\kappa=1,5$ for structural carbon steel;

$\kappa=1,4$ for low alloy steel; and

$\kappa=1,1$ for quenched and tempered steel.

See also data in [6]. 


\section{Comments on probabilistic models}

\subsection{Actions}

\section{Permanent load}

The characteristic value of self-weight is in general determined from nominal dimensions (which are normally equal to the mean dimensions) and from the mean densities. So, in many common cases the mean of self-weight is approximately equal to its nominal (characteristic) value. The actual dimensions of structural members may, however, differ (by several per cent) from their nominal value depending on the construction material and production as indicated in [6]. The coefficient of variation of weight density (and self-weight) varies in common cases from 0,03 up to $0,10[6,8]$ as indicated in Table 1 .

Self-weight $G$ of structural members may usually be determined as a product of the volume $\Omega$ and density $\gamma$.

$$
G=\Omega \gamma
$$

Both the volume $\Omega$ and the density $\gamma$ are random variables that may be described by a normal distribution [6]. The mean of the volume $\Omega$ is approximately equal to the nominal value (as a rule slightly greater), the mean of the density $\gamma$ is usually well defined by the producer. Informative coefficients of variation are indicated in Table 1a; more extensive data are available in [6].

The coefficient of variation $w_{G}$ of the resulting self-weight may be estimated as

$$
w_{G}^{2}=w_{\Omega}^{2}+w_{\gamma}^{2}+w_{\Omega}^{2} w_{\gamma}^{2}
$$

The last term in equation (3) may be usually neglected.

Table 1a. Examples of the coefficients of variation (indicative values only)

\begin{tabular}{|l|c|c|c|}
\hline \multirow{2}{*}{ Material } & \multicolumn{3}{|c|}{ Coefficient of variation of } \\
\cline { 2 - 4 } & $\Omega$ & $\gamma$ & $G$ \\
\hline Steel (rolled) & 0.03 & 0.01 & 0.031 \\
\hline Concrete (plate 300 mm thick, ordinary) & 0.02 & 0.04 & 0.045 \\
\hline Masonry unplastered & 0.04 & 0.05 & 0.080 \\
\hline Timber (sawn beam 200 mm thick, dry) & 0.01 & 0.10 & 0.100 \\
\hline
\end{tabular}

Data indicated in Table 1a should be considered as informative values only. The coefficients of variation of $\Omega$ for concrete and timber depend strongly on the size (increase with decreasing thickness of members) and type of material. Note also that variability of nonstructural members may be considerably greater than self-weight of structural members (see also [8]).

\section{Imposed load}

The imposed load considered in Table 1 refers to office areas for which the characteristic value recommended in EN 1991-1 [2], is within the range from 2 to $3 \mathrm{kN} / \mathrm{m}^{2}$. The experimentally determined mean of sustained load is $0,5 \mathrm{kN} / \mathrm{m}^{2}$, which is approximately 0,2 of the mean, as indicated in Table 1 . The standard deviation may vary in a broad range depending on the loaded area, influence coefficients and other factors as described in $[6,9]$. 
The coefficient of variation 1,1 corresponds approximately to a loaded area of $50 \mathrm{~m}^{2}$ and influence coefficient 1,4 . Note that with increasing area the coefficient of variation decreases.

Thus, the parameters of the imposed load indicated in Table 1 are derived considering typical office areas. Nevertheless, they may also be used as a first approximation (a priori information) for imposed loads in other types of loaded areas.

When time-variant analysis is applied, the imposed load is usually split into a sustainable (long-term) part and a short-term part. Parameters of both components of imposed load (including jump rate $\lambda$ and interarrival duration intensity $\rho$ ) should be taken from available documents $[6,9]$.

Imposed load $Q$ is usually described by Gumbel distribution (in [6] also Gamma and exponential distribution is used for sustained and intermittent load respectively). In general, the total imposed load $Q$ consists of the sustained (long-term) component $q$ and intermittent (short-term) component $p$. The sustained load $q$ is always present while the intermittent component $p$ may be absent and in fact may be active only very rarely (for example a few days a year only). The parameters of both components including jump rate $\lambda$ of sustained load, $v$ jump rate of intermittent load and $d$ duration time of intermittent load are indicated in Table $1 \mathrm{~b}$, which is taken from JCSS materials [6].

Table 1b. Parameters of the imposed load in accordance with loading areas.

\begin{tabular}{|c|c|c|c|c|c|c|c|c|c|}
\hline \multirow{2}{*}{ Category } & \multirow{2}{*}{$\begin{array}{c}A_{0} \\
{\left[\mathrm{~m}^{2}\right]}\end{array}$} & \multicolumn{4}{|c|}{ Sustained load $q$} & \multicolumn{4}{|c|}{ Intermittent load $p$} \\
\hline & & $\begin{array}{c}\mu_{q} \\
{\left[\mathrm{kN} / \mathrm{m}^{2}\right]}\end{array}$ & $\begin{array}{c}\sigma_{V} \\
{\left[\mathrm{kN} / \mathrm{m}^{2}\right]}\end{array}$ & $\begin{array}{c}\sigma_{U} \\
{\left[\mathrm{kN} / \mathrm{m}^{2}\right]}\end{array}$ & $\begin{array}{c}1 / \lambda \\
\text { [years] }\end{array}$ & $\begin{array}{c}\mu_{p} \\
{\left[\mathrm{kN} / \mathrm{m}^{2}\right]}\end{array}$ & $\begin{array}{c}\sigma_{U} \\
{\left[\mathrm{kN} / \mathrm{m}^{2}\right]}\end{array}$ & $\begin{array}{c}1 / v \\
\text { [years] }\end{array}$ & $\begin{array}{c}d \\
\text { [days] }\end{array}$ \\
\hline Office & 20 & 0,5 & 0,3 & 0,6 & 5 & 0,2 & 0,4 & 0,3 & $1-3$ \\
\hline Lobby & 20 & 0,2 & 0,15 & 0,3 & 10 & 0,4 & 0,6 & 1 & $1-3$ \\
\hline Residence & 20 & 0,3 & 0,15 & 0,3 & 7 & 0,3 & 0,4 & 1 & $1-3$ \\
\hline Hotel rooms & 20 & 0,3 & 0,05 & 0,1 & 10 & 0,2 & 0,4 & 0,1 & $1-3$ \\
\hline Patient room & 20 & 0,4 & 0,3 & 0,6 & $5-10$ & 0,2 & 0,4 & 1 & $1-3$ \\
\hline Laboratory & 20 & 0,7 & 0,4 & 0,8 & $5-10$ & & & & \\
\hline Libraries & 20 & 1,7 & 0,5 & 1 & 10 & & & & \\
\hline Classroom & 100 & 0,6 & 0,15 & 0,4 & 10 & 0,5 & 1,4 & 0,3 & $1-5$ \\
\hline $\begin{array}{l}\text { Stores } \\
\text { first floor } \\
\text { upper floor }\end{array}$ & $\begin{array}{l}100 \\
100\end{array}$ & $\begin{array}{l}0,9 \\
0,9\end{array}$ & $\begin{array}{l}0,6 \\
0.6\end{array}$ & $\begin{array}{l}1,6 \\
1.6\end{array}$ & $\begin{array}{l}1-5 \\
1-5\end{array}$ & $\begin{array}{l}0,4 \\
0,4\end{array}$ & $\begin{array}{l}1,1 \\
1,1\end{array}$ & $\begin{array}{l}1,0 \\
1,0\end{array}$ & $\begin{array}{l}1-14 \\
1-14\end{array}$ \\
\hline Storage & 100 & 3,5 & 2,5 & 6,9 & $0,1-1$ & & & & \\
\hline $\begin{array}{l}\text { Industrial } \\
\text { - light } \\
\text { - heavy }\end{array}$ & $\begin{array}{l}100 \\
100\end{array}$ & $\begin{array}{l}1 \\
3\end{array}$ & $\begin{array}{c}1 \\
1,5\end{array}$ & $\begin{array}{l}2,8 \\
4,1\end{array}$ & $\begin{array}{l}5-10 \\
5-10\end{array}$ & & & & \\
\hline $\begin{array}{l}\text { Concentration } \\
\text { of peoples }\end{array}$ & 20 & & & & & 1,25 & 2,5 & 0,02 & 0,5 \\
\hline
\end{tabular}

The standard deviation of the sustained load $q$ may be determined [6] as

$$
\sigma_{q}^{2}=\sigma_{V}^{2}+\sigma_{U}^{2} \frac{A_{0}}{A} \kappa
$$

where $\sigma_{V}$ is the standard deviation of the overall load intensity, $\sigma_{U}$ is the standard deviation of the random field describing space variation of the load, $A_{0}$ denotes the reference area (20 or $100 \mathrm{~m}^{2}$ ), $A$ the loaded area and $\kappa$ is the influence factor depending on the structural arrangement including boundary conditions. In common cases the factor $\kappa$ is within the 
interval from 1 to 2,4 [6]. Figure 1 shows typical influence lines and corresponding factors $\kappa$ ( $\kappa=2$ is considered in the following example as a representative value).

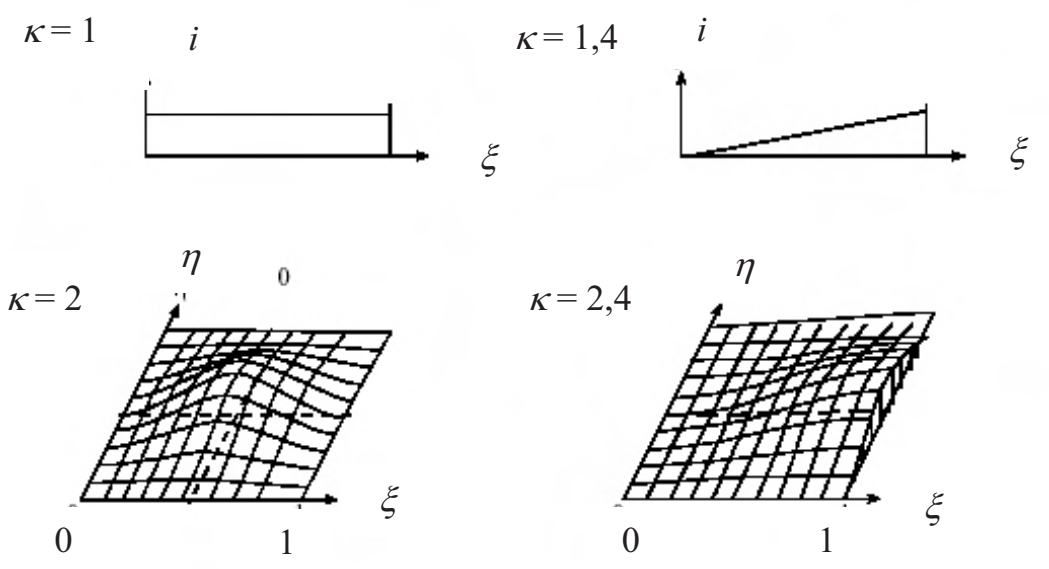

Figure 1. Typical influence lines and corresponding factors $\kappa$.

A relationship similar to equation (5) may be used to determine the standard deviation $\sigma_{p}$ of the intermittent load $p$.

As an example consider an office area for which the characteristic value $Q_{\mathrm{k}}=3 \mathrm{kN} / \mathrm{m}^{2}$ is recommended in [3]. In accordance with Table 2 the mean values $\mu_{q}$ (for a 5 -year period) and $\mu_{p}$ (for a 1 to 3-day period) are

$$
\mu_{q, 5}=0,5 \mathrm{kN} / \mathrm{m}^{2}, \mu_{p}=0,2 \mathrm{kN} / \mathrm{m}^{2}
$$

Assuming the factor $\kappa=2$ and the loaded area $A=40 \mathrm{~m}^{2}$, the standard deviations are as follows

$$
\sigma_{q}=\left(0,30^{2}+0,60^{2} \times 2 \times 20 / 40\right)^{0.5}=0,67 \mathrm{kN} / \mathrm{m}^{2}, \sigma_{p}=\left(0,40^{2} \times 2 \times 20 / 40\right)^{0.5}=0,40 \mathrm{kN} / \mathrm{m}^{2}
$$

Note that the standard deviations are strongly dependent on the factor $\kappa$ and the loading area $A=40 \mathrm{~m}^{2}$ loaded area $A$; if $\kappa=2$ and $\mathrm{A}=20 \mathrm{~m}^{2}$ then

$$
\sigma_{q}=\left(0,30^{2}+0,60^{2} \times 2 \times 20 / 20\right)^{0.5}=0,90 \mathrm{kN} / \mathrm{m}^{2}, \sigma_{p}=\left(0,40^{2} \times 2 \times 20 / 20\right)^{0.5}=0,57 \mathrm{kN} / \mathrm{m}^{2}
$$

In general, with increasing loaded area $A$ the standard deviations of both load components decreases.

Considering the factor $\kappa=2$ and the loaded area $A=40 \mathrm{~m}^{2}$ and assuming Gumbel distribution for the sustained load $p$, then the 50 -year extremes have the mean $m_{p, 50}$ and coefficient of variation $w_{p, 50}$

$$
\mu_{p, 50}=0,5+0,78 \ln (10 \times 0,67)=1,98 \mathrm{kN} / \mathrm{m}^{2}, w_{p, 50}=0.34
$$

Indicative parameters $\mu_{p, 50} / Q_{\mathrm{k}}=0,6$ and $w_{p}=0.35$ correspond well to the above data and may therefore be chosen for a first approximation. 


\section{Wind load}

Statistical parameters of wind pressure $w(z)$ indicated in Table 1a are derived considering the EN model [3] and JCSS probabilistic model code [6]. Assuming oropraphy factor equal to 1 , wind pressure $w(z)$ can be written as:

$$
w(z)=c_{\mathrm{p}} c_{\mathrm{g}}(z) c_{\mathrm{r}}(z)^{2} m_{\mathrm{q}} q_{\mathrm{b}}, c_{\mathrm{g}}(z)=1+7 I_{\mathrm{v}}(z), \quad q_{\mathrm{b}}=\frac{1}{2} \rho v_{\mathrm{b}}^{2}
$$

Here $c_{\mathrm{p}}$ denotes the pressure coefficient, which depends on geometry of a structure and the size of a loaded area, $c_{\mathrm{g}}(z)$ denotes the gust factor [6] (for terrain category II and $z \cong 7,5 \mathrm{~m}$ equal to 2,4), which depends on the turbulence intensity $I_{\mathrm{v}}(z)$ defined in [3], $\mathrm{c}_{\mathrm{r}}(z)$ is the roughness factor defined in [3] (for terrain category II and $z \cong 7,5 \mathrm{~m}$ equal to 0,95 ), $m_{\mathrm{q}}$ is the model coefficient introduced in [6], which describes the ratio between expected and computed values of the basic wind pressure $q_{\mathrm{b}}, \rho$ denotes the air density (assumed as $\rho=1,25 \mathrm{~kg} / \mathrm{m}^{2}$ ) and $v_{\mathrm{b}}$ is the reference wind speed specified in maps for a given region (assumed as $v_{\mathrm{b}}=26$ $\mathrm{m} / \mathrm{s})$.

Table $1 \mathrm{c}$. Wind load parameters assuming terrain category II and $\mathrm{z} \cong 7,5 \mathrm{~m}$, the reference wind speed $v_{\mathrm{b}}=26 \mathrm{~m} / \mathrm{s}$ and its coefficient of variation 0,20 .

\begin{tabular}{|c|c|c|c|c|c|c|c|c|c|}
\hline \multicolumn{10}{|c|}{$w=c_{\mathrm{p}} c_{\mathrm{g}} c_{\mathrm{r}}^{2} m_{\mathrm{q}} q_{\mathrm{b}}$} \\
\hline \multirow{2}{*}{$\begin{array}{l}\text { Var. } \\
\text { types }\end{array}$} & \multirow{2}{*}{$\begin{array}{c}\text { Symbol } \\
X \\
\end{array}$} & \multirow{2}{*}{ Name of basic variable } & \multirow{2}{*}{ Dist. } & \multirow{2}{*}{ Dim. } & \multicolumn{5}{|c|}{ Parameters } \\
\hline & & & & & $X_{\mathrm{k}}$ & $\mu_{X}$ & $\mu_{X} / X_{\mathrm{k}}$ & $\sigma_{X}$ & $w_{X}$ \\
\hline \multirow{4}{*}{ ن } & $c_{\mathrm{p}}$ & Pressure coefficient & $\mathrm{N}$ & & nom & nom & 1 & 0,1 nom & 0,1 \\
\hline & $c_{\mathrm{g}}$ & Gust factor & $\mathrm{N}$ & - & 2,4 & 2,4 & 1 & 0,24 & 0,1 \\
\hline & $c_{\mathrm{r}}^{2}$ & Roughness factor & $\mathrm{N}$ & - & 0,91 & 0,73 & 0,8 & 0,073 & 0,1 \\
\hline & $m_{\mathrm{q}}$ & Model coefficient & $\mathrm{N}$ & - & 1 & 0,8 & 0,8 & 0,16 & 0,2 \\
\hline \multirow{4}{*}{ 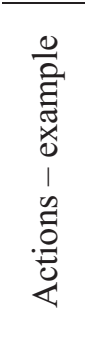 } & $q_{\mathrm{b}, 1}$ & $\begin{array}{c}\text { Annual extremes of basic } \\
\text { wind pressure }\end{array}$ & G & $\mathrm{kN} / \mathrm{m}^{2}$ & $0,42 *$ & $0,20 *$ & 0,44 & 0,085 & 0,43 \\
\hline & $q_{\mathrm{b}, 50}$ & $\begin{array}{c}50 \text {-year extremes of basic } \\
\text { wind pressure }\end{array}$ & G & $\mathrm{kN} / \mathrm{m}^{2}$ & - & 0,46 & 1,10 & 0,085 & 0,18 \\
\hline & $w_{1}$ & $\begin{array}{c}\text { Annual extremes of wind } \\
\text { pressure }\end{array}$ & G & $\mathrm{kN} / \mathrm{m}^{2}$ & 0,92 & 0,28 & 0,30 & 0,15 & 0,50 \\
\hline & $W_{50}$ & $\begin{array}{l}50 \text {-year extremes of wind } \\
\text { pressure }\end{array}$ & G & $\mathrm{kN} / \mathrm{m}^{2}$ & - & 0,64 & 0,70 & 0,211 & 0,33 \\
\hline
\end{tabular}

Notes: * The characteristic value of $q_{\mathrm{b}, 1}$ is determined for the wind speed $v_{\mathrm{b}}=26 \mathrm{~m} / \mathrm{s}$.

** The mean of $q_{\mathrm{b}, 1}$ is determined assuming Gumbel distribution, coefficient of variation of the wind speed 0,2 and probability 0,02 of the characteristic value being exceeded.

Note that the product $c_{\mathrm{e}}(z)=c_{\mathrm{g}}(z) c_{\mathrm{r}}(z)^{2}$ is defined in [3] as the exposure factor, which is given for various terrain categories in a diagram. The annual extremes of basic wind pressure is denoted $q_{\mathrm{b}, 1}$.

Table 1c shows the statistical parameters of all coefficients and resulting wind actions assuming the reference wind speed $v_{\mathrm{b}}=26 \mathrm{~m} / \mathrm{s}$ and its coefficient of variation 0,20 , which is a middle value (it may vary from 0,10 up to $0,35[6]$ ). The characteristics for $w_{1}$ and $w_{50}$ determined in Table $1 \mathrm{c}$ considering a middle value 0,20 are also included in Table 1. 
Parameters of time-variant behaviour of wind load (jump rate $\lambda$ and interarrival duration intensity $\rho$ ) should be taken from available documents $[6,11]$.

\section{Snow load}

Statistical parameters of the snow load indicated in Table $1 \mathrm{~d}$ are derived considering the EN model [4], which can be written as:

$$
s=\mu_{i} C_{\mathrm{e}} C_{\mathrm{t}} s_{\mathrm{g}}
$$

where $\mu_{i}$ is the load shape coefficient considered for a uniform snow load covering a whole roof area and for the roof slope about $15^{\circ}, C_{\mathrm{e}}$ denotes the exposure coefficient and $C_{\mathrm{t}}$ denotes the heat coefficient. The snow load on ground at the weather station $s_{\mathrm{g}}$ is specified in maps for a given region.

Table 1d shows the statistical parameters of these coefficients and resulting snow actions assuming a middle value of the coefficient of variation of annual extremes of snow load on ground $s_{\mathrm{g}, 1}$ may vary from 0,3 to 1,15 [6]. The characteristics for $s_{1}$ and $s_{50}$ determined in Table $1 \mathrm{~d}$ assuming a middle value 0,70 are also included (using rounded values) in summary Table 1 .

Table 1d. Snow load parameters assuming the coefficient of variation for snow load on grounds $s_{\mathrm{g}, 1}$ equal to 0,70 .

\begin{tabular}{|c|c|c|c|c|c|c|c|c|c|}
\hline \multicolumn{10}{|c|}{$s=\mu_{1} C_{\mathrm{e}} C_{\mathrm{t}} s_{\mathrm{g}}$} \\
\hline \multirow{2}{*}{$\begin{array}{l}\text { Var. } \\
\text { types }\end{array}$} & \multirow{2}{*}{$\begin{array}{c}\text { Symbol } \\
X \\
\end{array}$} & \multirow{2}{*}{ Name of basic variable } & \multirow{2}{*}{ Dist. } & \multirow{2}{*}{ Dim. } & \multicolumn{5}{|c|}{ Parameters } \\
\hline & & & & & $X_{\mathrm{k}}$ & $\mu_{X}$ & $\mu_{X} / X_{\mathrm{k}}$ & $\sigma_{X}$ & $w_{X}$ \\
\hline \multirow{2}{*}{$\begin{array}{l}\dot{4} \\
\dot{0} \\
ن\end{array}$} & $\mu_{1} C_{\mathrm{e}}$ & Shape and exposure coef. & $\mathrm{N}$ & - & 0,8 & 0,8 & 1 & 0,12 & 0,15 \\
\hline & $C_{\mathrm{t}}$ & Heat coefficient & $\mathrm{D}$ & - & 1 & 1 & 1 & - & - \\
\hline \multirow{4}{*}{ 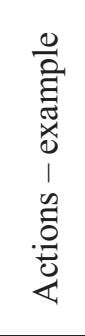 } & $s_{\mathrm{g}, 1} *$ & $\begin{array}{l}\text { Annual extremes of snow } \\
\text { load on ground }\end{array}$ & G & $\mathrm{kN} / \mathrm{m}^{2}$ & 1,33 & 0,47 & 0,35 & 0,33 & 0,70 \\
\hline & $s_{\mathrm{g}, 50}$ & $\begin{array}{l}50 \text {-year extremes of snow } \\
\text { load on ground }\end{array}$ & G & $\mathrm{kN} / \mathrm{m}^{2}$ & - & 1,48 & 1,11 & 0,33 & 0,22 \\
\hline & $s_{1}$ & $\begin{array}{c}\text { Annual extremes of snow } \\
\text { load on roof }\end{array}$ & G & $\mathrm{kN} / \mathrm{m}^{2}$ & 1,06 & 0,38 & 0,35 & 0,25 & 0,72 \\
\hline & $s_{50}$ & $\begin{array}{c}50 \text {-year extremes of snow } \\
\text { load on roof }\end{array}$ & $\mathrm{G}$ & $\mathrm{kN} / \mathrm{m}^{2}$ & - & 1,18 & 1,11 & 0,33 & 0,28 \\
\hline
\end{tabular}

Note: * The mean of sg,1 is determined assuming Gumbel distribution, coefficient of variation of the snow on ground 0,7 and probability 0,02 of the characteristic value being exceeded.

It should be noted that the coefficient of variation of annual extremes of snow on ground may vary in a broad range from 0,30 up to 1,15 [6] depending on local conditions (see coast, inland and mountains regions).

Parameters of time-variant behaviour of snow load (jump rate $\lambda$ and interarrival duration intensity $\rho$ ) should be taken from available documents $[6,10]$. 


\subsection{Material properties}

The mean value $\mu_{X}$ of a material property $X$ (the yield point for common steel of the grade S 235 and S 355, normal concrete strength and reinforcing bars) may be estimated using a simple expression

$$
\mu_{X}=X_{\mathrm{k}}+k \sigma_{X}=X_{\mathrm{k}} /\left(1-k w_{X}\right)
$$

where $k$ (usually equal to 2 ) is a coefficient taking into account quality control procedure, $\sigma_{X}$ denotes the standard deviation and $w_{X}$ the coefficient of variation of $X$. The mean of ultimate strength is usually determined from the mean of the yield point using factor $\kappa[6]$ as indicated in Table 1. The coefficient of variation $w_{X}$ for structural steel is within the range from 0,7 to 0,10 , for ultimate strength is around 0,05 [6]. For concrete and reinforcing bars usually the standard deviation $\sigma_{X}$ (commonly 5 and $30 \mathrm{kN} / \mathrm{m}^{2}$ respectively) is considered instead of the coefficient of variation $w_{X}$. However, the coefficient of variation of concrete may vary in a broad range 0,05 to 0,18 depending on the production procedure.

Note that an alternative expression for the mean $\mu_{X}$ of yield point of structural steel may be found in literature (see [6] and references indicated there). The following expression is offered in [6] for structural steel (rolled sections):

$$
\mu_{X}=X_{\mathrm{k}} \alpha \exp \left(k w_{X}\right)-C
$$

In this expression $\alpha$ denotes spatial position factor ( $\alpha=1,05$ for webs of hot rolled section, $\alpha=1,00$ otherwise), $C$ is a constant reducing the yield strength as obtained from usual mill tests to the static yield strength (a value $20 \mathrm{MPa}$ is recommended but rate of loading should be considered).

A comparison of expressions (12) and (13) indicates that expression (13) is more conservative (primarily due to the reduction constant $C$ ). For steel S 355 the mutual differences may be about 5\%. However, recent experience with a great number of experimental data clearly indicates that the second expression (13) is rather conservative (and unrealistic for small coefficients of variation) while the first expression (12) appears to be more suitable and is therefore recommended here. However, even expression (12) may be conservative in some cases. Nevertheless, any such expression is always an approximation providing informative (a priori information) values only, which should be updated in particular conditions using long-term experience and available experimental data.

\subsection{Geometric data}

Variability of geometric data is usually less significant than variability of material properties. Statistical characteristics indicated in Table 1 are taken directly from available data $[6,16]$ and other measurements. Note that the mean of steel cross-sections in the case of $\mathrm{H}-$ profiles is $0.99 X_{\text {nom, }}$, and slightly greater $(1,02)$ in case of $\mathrm{L}$ profiles and rods, with coefficient of variation around a value of $3 \%$. In general variation of dimensions in the case of reinforced concrete cross-section is more significant (standard deviation from 5 to $10 \mathrm{~mm}$ ) than variation of steel sections.

In particular variation of the concrete cover of reinforced concrete cross-sections may be important depending on the type and size of cross-section. Both-sided, limited Beta distribution (or Gamma distribution) seems to be the most suitable theoretical model in this case. Data indicated in Table 1 correspond to middle values of expected ranges [6]. In the case of Beta distribution the lower bound can be assumed to be zero, $a=0$, the mean equal to the nominal (design) value, $\mu_{x}=x_{\text {nom }}$, and the upper bound equal to three times the mean, $b=$ $3 \mu_{x}$, which corresponds to a skewness equal to two times the coefficient of variation, $\alpha_{x}=2 w_{x}$ (which is characteristic for Gamma distribution). 


\subsection{Model uncertainties}

Model uncertainties of action effects and resistances are conventionally expressed through the coefficients defined as ratio of the observed and model values [6]. In some cases little data are available and often professional judgments and experience are taken into consideration. Information given in Table 1 is adopted from the latest documents of JCSS [6]. Model uncertainty coefficients for resistances of steel and concrete members are provided in more detail in Table 2 and 3. Table 4 indicates uncertainty coefficients for load effects. In all cases log-normal distribution having the origin at zero is recommended in [6].

Table 2. Coefficient of model uncertainties for resistance of steel members

\begin{tabular}{lcccc}
\hline Model type & Distribution & Mean & CoV & References \\
\hline Beam bending moment capacity & LN & 1,00 & 0,05 & 6 \\
Column resistance & LN & 1,20 & 0,10 & $*$ \\
Welded connection capacity & LN & 1,15 & 0,20 & 6 \\
Bolted connection capacity & LN & 1,25 & 0,08 & 6 \\
\hline
\end{tabular}

Note. * Data taken from previous materials of JCSS, which are not, however, included in the latest version of JCSS website [6].

Table 3. Coefficient of model uncertainties for resistance of concrete members

\begin{tabular}{lcccc}
\hline Model type & Distribution & Mean & CoV & References \\
\hline Bending moment capacity & LN & 1,20 & 0,15 & 6 \\
Shear capacity of beams & LN & 1,00 & 0,10 & 6 \\
Column resistance & LN & 1,20 & 0,15 & $*$ \\
Capacity of connections & LN & 1,00 & 0,15 & $*$ \\
Punching shear & LN & 1,00 & 0,15 & $*$ \\
\hline
\end{tabular}

Note. * Data taken from previous materials of JCSS, which are not, however, included in the latest version of JCSS website [6].

Table 4. Coefficient of model uncertainties for load effect.

\begin{tabular}{lcccc}
\hline Model type & Distribution & Mean & CoV & References \\
\hline Moment in frames & LN & 1,00 & 0,10 & 6 \\
Axial force in frame & LN & 1,00 & 0,05 & 6 \\
Shear forces in frame & LN & 1,00 & 0,10 & 6 \\
Moments in plates & LN & 1,00 & 0,20 & 6 \\
Forces in plates & LN & 1,00 & 0,10 & 6 \\
Stresses in 2D solids & LN & 1,00 & 0,05 & 6 \\
Stresses in 3D solids & LN & 1,00 & 0,05 & 6 \\
Deflection in steel structures & LN & 1,00 & 0,07 & $*$ \\
Deflection in concrete structures & LN & 1,00 & 0,10 & $*$ \\
Crack width in concrete & LN & 1,00 & 0,30 & $*$ \\
\hline
\end{tabular}

Note. * Data taken from previous materials of JCSS, which are not, however, included in the latest version of JCSS website [6]. 
Data indicated in Tables 2, 3 and 4 should be, however, considered as indicative values only and ought to be verified taking into account structural conditions and available experimental data. It is expected that coefficients of model uncertainties will be further developed by JCSS taking into account newly available experimental data and their statistical evaluation.

\subsection{Time-variant parameters}

Time-variant behaviour of climatic actions (snow and wind) is usually described by jump process with intermittencies. Figure 2 shows a possible approximation used to describe actual time-dependency of an action. Two basic parameters can be considered for each action as indicated schematically in Figure 2 [6]: jump rate $\lambda=1 / \mu_{\tau}$ and the mean duration $\mu_{\eta}$ of the action being "on". Using these two parameters the interarrival duration intensity $\rho$ is defined as

$$
\rho=\mu_{\eta} / \mu_{\tau}=\lambda \mu_{\eta}
$$

As for the snow load, let us assume that it takes its extreme five times a year $\left(\lambda_{\mathrm{s}}=5\right)$. Therefore, the arrival rate of on-times is also equal to 5 . The mean duration (the time during which the structure is loaded by the extreme snow load) is supposed to be about 14 days. The interarrival duration intensity results $\rho_{\mathrm{s}}=5 \times 14 / 365=0,19$.

As for the wind load, windstorms are expected to appear ten times a year $\left(\lambda_{\mathrm{w}}=10\right)$ and the mean duration of the storm is approximately 8 hours. The interarrival duration intensity is $\rho_{\mathrm{w}}=10 / 365 / 3=0,009$.

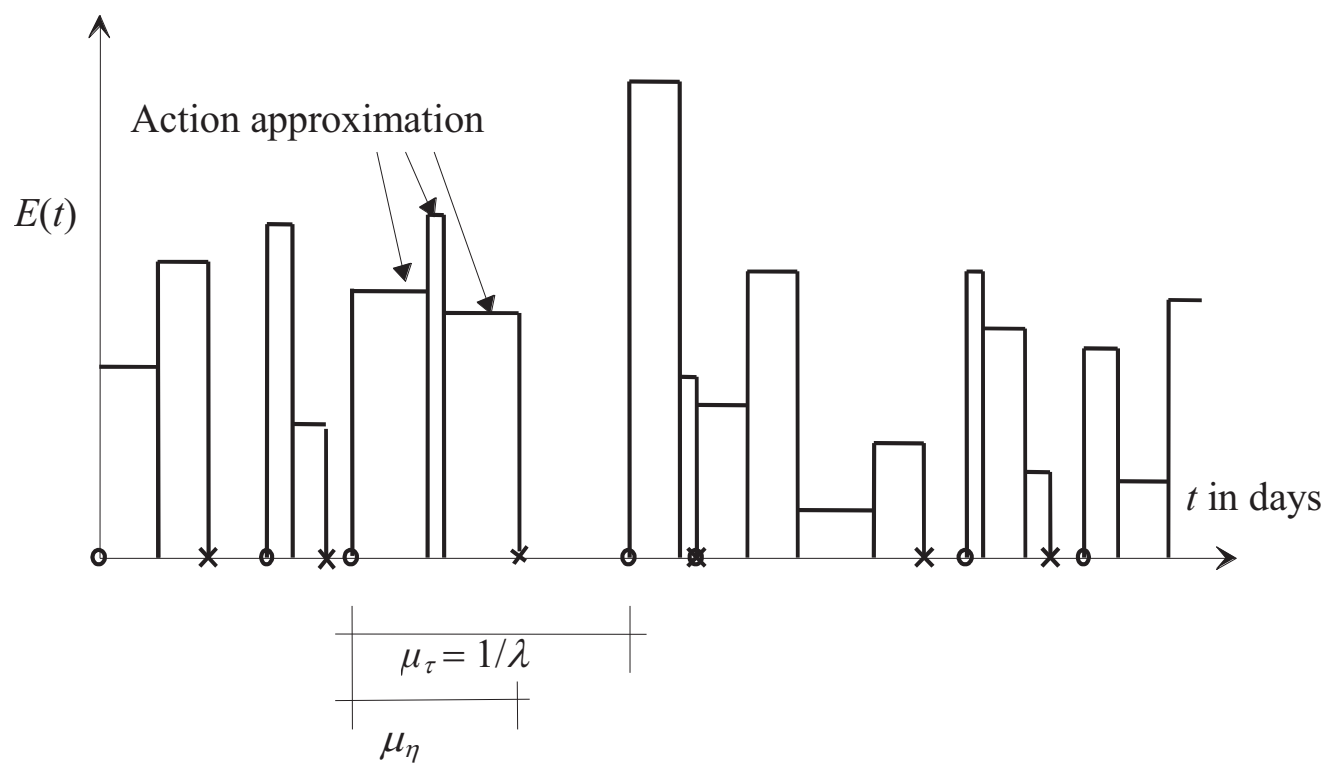

Figure 2. Jump process with intermittencies

The above-mentioned procedure for determination of the interarrival duration intensity ratio $\rho$ for selected values of the jump rate $\lambda$ and the mean duration $\mu_{\eta}$ (corresponding to climatic actions) is illustrated in the following Table 5. 
Table 5. The interarrival duration intensity ratio $\rho$ for selected values of the jump rate $\lambda$ and the mean duration $\mu_{\eta}$ (corresponding to climatic actions).

\begin{tabular}{c|cccccccc}
$\lambda$ & \multicolumn{8}{c}{ The mean duration $\mu_{\eta}[$ hours $]$} \\
[1/years] & 4 & 8 & 12 & 16 & 24 & 48 & 72 & 144 \\
\cline { 2 - 9 } & 0,0005 & 0,0009 & 0,0014 & 0,0018 & 0,0027 & 0,0055 & 0,0082 & 0,0164 \\
1 & 0,0005 & 0,0009 & 0,0014 & 0,0018 & 0,0027 & 0,0055 & 0,0082 & 0,0164 \\
2 & 0,0009 & 0,0018 & 0,0027 & 0,0037 & 0,0055 & 0,0110 & 0,0164 & 0,0329 \\
4 & 0,0018 & 0,0037 & 0,0055 & 0,0073 & 0,0110 & 0,0219 & 0,0329 & 0,0658 \\
6 & 0,0027 & 0,0055 & 0,0082 & 0,0110 & 0,0164 & 0,0329 & 0,0493 & 0,0986 \\
8 & 0,0037 & 0,0073 & 0,0110 & 0,0146 & 0,0219 & 0,0438 & 0,0658 & 0,1315 \\
10 & 0,0046 & 0,0091 & 0,0137 & 0,0183 & 0,0274 & 0,0548 & 0,0822 & 0,1644 \\
12 & 0,0055 & 0,0110 & 0,0164 & 0,0219 & 0,0329 & 0,0658 & 0,0986 & 0,1973 \\
14 & 0,0064 & 0,0128 & 0,0192 & 0,0256 & 0,0384 & 0,0767 & 0,1151 & 0,2301 \\
18 & 0,0082 & 0,0164 & 0,0247 & 0,0329 & 0,0493 & 0,0986 & 0,1479 & 0,2959 \\
24 & 0,0110 & 0,0219 & 0,0329 & 0,0438 & 0,0658 & 0,1315 & 0,1973 & 0,3945
\end{tabular}

Typical jump arrival rates $\lambda$, the mean duration $\mu_{\eta}$ and the interarrival duration intensity $\rho$ are indicated in [6]. Indicative values of the jump rate $\lambda$ and the mean duration $\mu_{\eta}$ based on data provided in [6] and judgement are given in Table 6.

Table 6. Recommended values of the jump rate $\lambda$ and the mean duration $\mu_{\eta}$.

\begin{tabular}{|l|c|c|c|c|}
\hline Location & \multicolumn{2}{|c|}{ Wind } & \multicolumn{2}{c|}{ Snow } \\
\hline Location - climate & $\begin{array}{c}\text { Jump rate } \lambda \\
{[1 / \text { year }]}\end{array}$ & $\begin{array}{c}\text { Duration } \mu_{\eta} \\
\text { [hours] }\end{array}$ & $\begin{array}{c}\text { Jump rate } \lambda \\
{[1 / \text { year }]}\end{array}$ & $\begin{array}{c}\text { Duration } \mu_{\eta} \\
\text { [hours] }\end{array}$ \\
\hline Inland climate & 5 to 15 & 4 to 24 & 2 to 5 & 24 to 144 \\
\hline Oceanic climate & 10 to 20 & 8 to 48 & 1 to 3 & 12 to 72 \\
\hline Mountain climate & 12 to 24 & 12 to 72 & 12 to 24 & 24 to 144 \\
\hline
\end{tabular}

The indicative values given in Table 6 should be considered as informative values only. In particular conditions both parameters, the jump rate $\lambda$ and the mean duration $\mu_{\eta}$, should be specified using available local information. In the case of a lack of representative information the lower and upper bounds of both parameters should be used to assess the possible effect of this uncertainty on the resulting reliability level.

Typical jump rates $\lambda \square \square \square$ the mean duration $\mu_{\eta}$ and the interarrival duration intensity $\rho$ for inland climates are given in Table 7 . 
Table 7. Typical jump arrival rates $\kappa=\lambda$ the mean duration $\mu_{\eta}$ and the interarrival duration intensity $\rho$.

\begin{tabular}{|c|c|c|c|}
\hline \hline Action & $\begin{array}{c}\text { Arrival rate } \kappa=\lambda \\
{[1 / \text { year }]}\end{array}$ & $\begin{array}{c}\text { Duration } \mu_{\eta} \\
{[\text { year }]}\end{array}$ & $\begin{array}{c}\text { Interarrival duration } \\
\text { intensity } \rho=\lambda \mu_{\eta}\end{array}$ \\
\hline \hline Snow & $5(5$ times a year $)$ & $14 / 365(14$ days $)$ & 0,19 \\
\hline Wind & $10(10$ times a year $)$ & $1 / 365 / 3(8$ hours $)$ & 0,009 \\
\hline
\end{tabular}

In particular examples it is necessary to take into account local climatic conditions. If the local conditions are unknown then it is recommended to assess a "lower" and "upper" bound of appropriate parameters and to verify their effect on the resulting reliability level.

\section{$4 \quad$ Concluding remarks}

Proposed conventional models of basic variables indicated above should be considered as indicative values only, valid in normal conditions only, which are intended to be used primarily for comparative studies. In a reliability analysis of a particular structure probabilistic models of basic variables should be specified taking into account actual loading and structural conditions, and relevant experimental data. When considering the proposed models the following remarks should be taken into account in particular.

1. Actual variability of self-weight and other permanent load may vary in a broad interval from 0.03 up to 0,10 ; it appears that the permanent load may be described by a normal distribution having the indicative values of the mean and coefficient of variation given as follows

$$
\mu_{G} / G_{\mathrm{k}}=1 \text { and } \mathrm{w}_{G}=0,1
$$

2. The imposed load may be described by Gumbel distribution having the indicative mean and coefficient of variation

$$
\mu_{p, 50} / Q_{\mathrm{k}}=0,6 \text { and } \mathrm{w}_{Q}=0.35
$$

Probabilistic models for imposed actions indicated in Table 1 correspond to an office area of middle size and middle level of action variability.

3. Wind actions may be described by Gumbel distribution having the indicative mean and coefficient of variation

$$
\mu_{p, 50} / Q_{\mathrm{k}}=0,7 \text { and } w_{Q}=0.35
$$

4. Snow loads may be described by Gumbel distribution having the indicative mean and coefficient of variation

$$
\mu_{p, 50} / Q_{\mathrm{k}}=1,1 \text { and } w_{Q}=0.30
$$

5. Time-variant parameters of variable actions (in particular climatic actions) should be specified, taking into account local conditions.

6. Probabilistic models for material property and geometric data indicated in Table 1 correspond to normal quality control. 


\section{References}

[1] EN 1990: Basis of Structural Design. (Transformation ENV 1991-1, 1994) Brussels, 2002.

[2] EN 1991-1: Action on Structures. (Transformation ENV 1991-2-1, 1994) Brussels, 2002.

[3] prEN 1991-1-4: Eurocode 1: Actions on Structures - Wind Loads, Brussels, CEN 2004.

[4] EN 1991-1-3: Eurocode 1: Actions on Structures - Snow Loads, Brussels, CEN 2004.

[5] ISO 2394: General Principles on Reliability for Structures. Zurich, 1997.

[6] JCSS, Joint Committee for Structural Reliability: Probabilistic model codes. Working materials, 2001 (available on web pages: http://www.jcss.ethz.ch/).

[7] Vrouwenvelder T., JCSS Probabilistic Model Code. Proc.: Safety, Risk, and Reliability. IABSE, Malta, 2001, pp. 65-70.

[8] CIB Report, Publication 115, Action on Structures, Self-Weight loads, CIB 1989.

[9] CIB Report, Publication 116, Action on Structures, Live Loads in Buildings, CIB 1989.

[10] CIB Report, Publication 141, Action on Structures, Snow Load, CIB 1995.

[11] CIB Report, Draft of CIB W81 Publication, Action on Structures, Wind Load, CIB working material, 1995

[12] SAKO, Joint Committee of NKB and INSTA-B: Basis of Design of Structures. Proposal for Modification of Partial Safety Factors in Eurocodes. 1999.

[13] Sorensen J.D., Hansen S.O. and Nielsen T.A., Partial Safety Factors and Target Reliability Level in Danish Codes. Proc.: Safety, Risk, and Reliability. IABSE, Malta, 2001, pp. 179-184.

[14] Holický M. and Marková J., Verification of load factors for concrete components by reliability and optimization analysis: Background documents for implementing Eurocodes. Progress in Structural Engineering and Materials, Vol. 2 No. 4, 2000, pp. 502-507.

[15] Caramelli S., Croce P., Salvatore W. and Sanpaolesi L., Partial safety factors for resistance of steel elements. University of Pisa, 1997.

[16] Miroslav Fajkus, Milan Holický, Lubomír Rozlívka and Miloš Vorlíček, Random Properties of Steel Elements Produced in Czech Republic, Eurosteel '99, Praha 1999, pp. 657-660.

[17] Ministry of the Environment, Housing and Building Department, Finland: Probabilistic Calibration of Partial Safety Factors (Eurocode and Finish proposal). 2000. 


\section{Annex 5: Partial factor method and probabilistic design}

\begin{tabular}{lr}
\multicolumn{1}{c}{ Contents } \\
1 Introduction & 175 \\
2 Fundamental load combinations & 175 \\
3 Partial factor design of a steel member & 177 \\
4 Probabilistic design of a steel member & 178 \\
5 Partial factor method and probabilistic design & 179 \\
6 Concluding remarks & 182
\end{tabular}

\section{Introduction}

Basic European document for structural design EN 1990 [1] allows the application of probabilistic methods as an alternative design procedure to the partial factor method. Basic instructions for probabilistic methods are provided in annexes C and D of [1] and in the International Standard ISO 2394 [2]. Further information can be found in specialised literature (for example [21, 22, 23]) or in JCSS materials [27]. A number of useful software products for the reliability analysis of structures (for example [24] and [25]) are commercially available and can be used effectively in practice. However, application and interpretation of results obtained using these products requires some experience and theoretical knowledge that may be found from simple examples.

The aim of this Annex is to supplement this design guide with basic guidance on the application of probabilistic methods using elementary mathematical tools. In addition the direct comparison of probabilistic design with the partial factor method is provided. For that purpose a system of special-purpose functions (described in an Annex to this contribution) has been developed using the language of technical computing MATLAB. The failure probability is computed using an approximate numerical integration. The system illustrates basic steps and practical procedures of the probabilistic design simultaneously with deterministic design based on the partial factor method.

It is believed that the MATLAB functions developed provide useful insight into probabilistic methods and enable the overcoming of difficulties with the correct interpretation of rather theoretical procedures. The direct comparison of the probabilistic design and partial factor methods may help in the understanding of mutual relations between input data of probabilistic methods and reliability elements (partial and various reduction factors) used by the partial factor method. A simple steel member exposed to a combination of one permanent and two variable actions is considered as an example illustrating general principles.

\section{$2 \quad$ Fundamental load combinations}

In the following, combinations of three actions, permanent action $G$, imposed load $Q$ (leading) and wind $W$ (accompanying), are considered. EN 1990 provides three combination rules in permanent and transient design situations denoted here "A", "B" and "C". Assuming the linear behaviour of structural members, actions $G, Q$ and $W$ and their characteristic values $G_{\mathrm{k}}, Q_{\mathrm{k}}$ and $W_{\mathrm{k}}$ denote appropriate load effects (not the original actions). The design value of 
the action effect $E_{\mathrm{d}}$ is generally obtained using the characteristic values $G_{\mathrm{k}}, Q_{\mathrm{k}}$ and $W_{\mathrm{k}}$ and appropriate partial factors $\gamma_{G}, \gamma_{Q}, \gamma_{W}$ and reduction factors $\xi, \psi_{Q}$ and $\psi_{W}$ as follows.

A. Considering the formula (6.10) in EN 1990, the design value of the action effect $E_{d}$ is given as

$$
E_{\mathrm{d}}=\gamma_{G} G_{\mathrm{k}}+\gamma_{Q} Q_{\mathrm{k}}+\gamma_{W} \psi_{W} W_{\mathrm{k}}
$$

B. An alternative procedure is provided in EN 1990 by twin expressions (6.10a) and (6.10b)

$$
\begin{gathered}
E_{\mathrm{d}}=\gamma_{G} G_{\mathrm{k}}+\gamma_{Q} \psi_{Q} Q_{\mathrm{k}}+\gamma_{W} \psi_{W} W_{\mathrm{k}} \\
E_{\mathrm{d}}=\xi \gamma_{G} G_{\mathrm{k}}+\gamma_{Q} Q_{\mathrm{k}}+\gamma_{W} \psi_{W} W_{\mathrm{k}}
\end{gathered}
$$

The less favourable action effect from equations (2) and (3) should be considered. In equation (3) $\xi$ is the reduction factor for unfavourable permanent actions $G$. Note that in equations (1) to (3) "+" generally implies "to be combined with".

C. In addition EN 1990 allows further modification of the alternative "B", simplifying equation (2) by considering permanent loads only, thus the load effect is then

$$
E_{\mathrm{d}}=\gamma_{G} G_{\mathrm{k}}
$$

The less favourable action effect resulting from (3) and (4) is then considered.

In the following only the first two combinations "A" and "B" are considered (combination "C" is similar to the combination "B"). The reliability elements (partial factors $\gamma_{G}, \gamma_{Q}, \gamma_{W}$ and the reduction factors $\xi, \psi_{Q}$ and $\psi_{W}$ ) recommended in EN 1990 [1] are applied. Factors $\gamma_{G}, \gamma_{Q}$ and $\gamma_{W}$ denote generally the partial factors of actions G, Q and W, though in accordance with EN 1990 [1] the partial factors for variable actions are equal, thus $\gamma_{Q}=\gamma_{W}$ ). Other possible combinations (as indicated in the handbook [16]) may be analysed similarly.

Note that if the leading action is wind W, then in equations (1) and (2) instead of reducing the wind action $W$ by factor $\psi_{W}$, the imposed load $Q$ should be reduced by the appropriate factor $\psi_{Q}$.

To enable easier generalisation of obtained results for a given load effect $E_{\mathrm{d}}$ under various intensities of variable actions, the characteristic values of $G_{\mathrm{k}}, Q_{\mathrm{k}}$ and $W_{\mathrm{k}}$ are related using quantities $\chi$ given as the ratio of variable actions $Q_{\mathrm{k}}+W_{\mathrm{k}}$ to the total load $G_{\mathrm{k}}+Q_{\mathrm{k}}+W_{\mathrm{k}}$, and the ratio k of accompanying action $W_{\mathrm{k}}$ to the main action $Q_{\mathrm{k}}$

$$
\chi=\left(Q_{\mathrm{k}}+W_{\mathrm{k}}\right) /\left(G_{\mathrm{k}}+Q_{\mathrm{k}}+W_{\mathrm{k}}\right), \quad k=W_{\mathrm{k}} / Q_{\mathrm{k}}
$$

Note that a realistic range of $\chi$ is from 0,1 to 0,6 (for a steel structure from 0,3 to 0,5 [16]). However, in some cases the load ratio $\chi$ may be very low if not zero (for example underground garages).

For a given design value of the load effect $E_{d}$ the characteristic values of individual actions $\mathrm{G}_{\mathrm{k}}, \mathrm{Q}_{\mathrm{k}}, \mathrm{W}_{\mathrm{k}}$ can be expressed using variables $\chi$ and $\mathrm{k}$ as follows

$$
G_{\mathrm{k}}=\frac{E_{\mathrm{d}}}{(\xi) \gamma_{G}+\frac{\left(\left(\psi_{Q}\right) \gamma_{Q}+k\left(\psi_{W}\right) \gamma_{W}\right) \chi}{(1+k)(1-\chi)}}, \quad Q_{\mathrm{k}}=\frac{\chi G_{\mathrm{k}}}{(1+k)(1-\chi)}, \quad W_{\mathrm{k}}=k Q_{\mathrm{k}}
$$

The factors $\xi, \psi_{Q}$ and $\psi_{W}$ indicated in the first relationship of (6) in brackets are applied in the same way (either yes or no) as in equations (1) to (4) for the alternative combination rules "A", "B" and "C". 
For the alternative choice "A", equation (1) is valid in the whole range $0 \leq \chi \leq 1$, whereas using alternative choice "B", equation (2) is valid in the interval $0 \leq \chi \leq \chi$ lim,B and equation (3) in the interval $\chi_{\lim , \mathrm{B}} \leq \chi \leq 1$. Correspondingly, for alternative choice "C", equation (4) is valid in the interval $0 \leq \chi \leq \chi_{\text {lim,C }}$ and equation (5) in the interval $\chi_{\text {lim,C }} \leq \chi \leq 1$. The limiting values $\chi_{\mathrm{lim}, \mathrm{B}}$ and $\chi_{\mathrm{lim}, \mathrm{C}}$ can be derived from equations (2) to (5) as follows

$$
\begin{gathered}
\chi_{\lim , \mathrm{B}}=\frac{\gamma_{G}(1-\xi)(1+k)}{\gamma_{G}(1-\xi)(1+k)+\gamma_{Q}\left(a-\psi_{Q}\right)+\gamma_{W} k\left(b-\psi_{W}\right)} \\
\chi_{\lim , \mathrm{C}}=\frac{\gamma_{G}(1-\xi)(1+k)}{\gamma_{G}(1-\xi)(1+k)+\gamma_{Q} a+\gamma_{W} k b}
\end{gathered}
$$

where the auxiliary variable $a=1$ and $b=\psi_{W}$ when $k \leq\left(1-\psi_{Q}\right) /\left(1-\psi_{W}\right)$ (imposed load $Q$ is the leading action) and $a=\psi_{Q}$ and $b=1$ when $k>\left(1-\psi_{Q}\right) /\left(1-\psi_{W}\right)$ (action $W$ is the leading action).

EN 1990 [1] allows through the National Annex, which will be published by the national standardisation institution

- which of the combination expression to use, and

- specification of appropriate safety factors

Thus, the National Annexes should include a recommendation for one of the alternatives indicated in EN 1990 for a fundamental combination of actions in the Ultimate limit states and partial factors $\gamma_{G}$ and $\gamma_{Q}$ for permanent and variable actions. Considering a generic structural member it may be shown that the choice of these nationally determined parameters might significantly affect the resulting reliability level. Partial and reduction factors $\gamma, \psi$ and $\xi$ recommended in EN 1990 [1] and used in this paper are summarised in Table 1 .

Table 1. Partial and reduction factors.

\begin{tabular}{|c|c|c|c|}
\hline Action & Partial factors $\gamma$ & Combination factor $\psi$ & Reduction factor $\xi$ \\
\hline \hline Permanent $G$ & 1,35 & 1,0 & 0.85 \\
\hline Imposed $Q$ & 1,5 & 0,7 & - \\
\hline Climatic $W$ & 1,5 & 0,6 & - \\
\hline
\end{tabular}

In addition to the factors indicated in Table 1 other information (for example characteristic values of actions) may be necessary to design a member using partial factor methods provided in EN 1990.

\section{Partial factor design of a steel member}

Design resistance $\mathrm{R}_{\mathrm{d}}$ of a steel member is described by a simple relationship

$$
R_{\mathrm{d}}=A f_{\mathrm{k}} / \gamma_{M}
$$

where $A$ denotes a design parameter depending on the geometry and boundary conditions of the member, $f_{\mathrm{k}}$ the characteristic strength, and $\gamma_{M}$ the partial factor of material property. Considering so-called economic design, when $E_{\mathrm{d}}=R_{\mathrm{d}}$, the design parameter $A$ is given as 


$$
A=E_{\mathrm{d}} \gamma_{M} / f_{\mathrm{k}}
$$

The partial factor of material property $\gamma_{M}$ is normally within the interval 1,0 to 1,15 (nationally determined parameter). Two alternatives $\gamma_{M}=1,00$ and $\gamma_{M}=1,10$ are considered in the attached numerical example of a steel member.

The load effect $E_{d}$ is given by equations (1) to (4) in terms of the characteristic values $G_{\mathrm{k}}, Q_{\mathrm{k}}, W_{\mathrm{k}}$ and appropriate reliability elements (the partial and reduction factors). The attached system of MATLAB functions considers the characteristic values $G_{\mathrm{k}}, Q_{\mathrm{k}}$ and $W_{\mathrm{k}}$, and further, the characteristic strength $f_{\mathrm{k}}$ and partial factor $\gamma_{M}$ as input data. The reliability elements (the partial and reduction factors) are considered by the default values recommended in EN 1990; nevertheless they may be easily adjusted in the inscription of the appropriate function if needed.

\section{$4 \quad$ Probabilistic design of a steel member}

The most important step in reliability analysis is the formulation of a limit state function (reliability margin) $\mathrm{G}(\boldsymbol{X})$ separating the safe and unsafe domain of basic variables $\boldsymbol{X}$. In this report the limit state function $\mathrm{G}(\boldsymbol{X})$ is considered in a simple form as the difference between the resistance $R(\boldsymbol{X})$ and the load effect $E(\boldsymbol{X})$

$$
\mathrm{G}(\boldsymbol{X})=R(\boldsymbol{X})-E(\boldsymbol{X})=\theta_{R} R_{0}(\boldsymbol{X})-\theta_{E} E_{0}(\boldsymbol{X})
$$

where the factor $\theta_{R}$ represents uncertainties of the resistance model $\mathrm{R}_{0}(\boldsymbol{X})$ and the factor $\theta_{E}$ represents uncertainties in the load effect model $\mathrm{E}_{0}(\boldsymbol{X})$. Note that the resistance $\mathrm{R}_{0}(\boldsymbol{X})$ in equation (11) corresponds to the resistance given by equation (9) and denoted there simply as $\mathrm{R}$, and the load effect $\mathrm{E}_{0}(\boldsymbol{X})$ is a probabilistic description of the load combination given in expressions (1) to (4). The vector $\boldsymbol{X}$ denotes all the basic variables entering the expressions for the resistance $\mathrm{R}_{0}(\boldsymbol{X})$ and the load effect $\mathrm{E}_{0}(\boldsymbol{X})$.

Considering the steel member described above, the following limit state function may be obtained from equations (9) and (11):

$$
\mathrm{G}(\boldsymbol{X})=\theta_{R} A f_{\mathrm{y}}-\theta_{E}\left(\mathrm{G}_{0}+\mathrm{Q}_{0}+\mathrm{W}_{0}\right)
$$

where vector $\boldsymbol{X}$ consists of seven basic variables (six random variables and one deterministic parameter) describing the resistance $\mathrm{R}$ and load effect $\mathrm{E}$.

Probabilistic methods of structural reliability then determine the failure probability $p_{\mathrm{f}}$, generally given by the integral

$$
p_{\mathrm{f}}=\mathrm{P}\{\mathrm{G}(\boldsymbol{X})<0\}=\int_{G(\boldsymbol{X}) \leq 0} \varphi(\boldsymbol{X}) \mathrm{d} \boldsymbol{X}
$$

where $\varphi(\boldsymbol{X})$ denotes the joint probability density function of the vector $\boldsymbol{X}$. As a rule instead of the failure probability $p_{\mathrm{f}}$ the reliability index $\beta$ is used as an equivalent reliability measure.

$$
\beta=-\Phi^{-1}\left(p_{\mathrm{f}}\right)
$$

where $\Phi^{-1}\left(p_{\mathrm{f}}\right)$ is the inverse distribution function of the standardised normal distribution.

As already mentioned in chapter 5 the integral in equation (13) indicates how the probability $p_{\mathrm{f}}$ can be determined provided that the joint probability density function $\varphi(\boldsymbol{X})$ is known (it may be a quite complicated or unknown function). In some special cases integration in equation (13) can be done analytically while, in some other cases, when the number of basic variables is small (up to 5), various types of numerical integration may be effectively applied. An approximate numerical integration is applied in the developed MATLAB functions. 
In the general case of many basic variables the failure probability $p_{\mathrm{f}}$ may be computed using various methods reviewed in chapter 2 . It should be mentioned again that approximate analytical methods including the FORM (First Order Reliability Method) method are very efficient reliability methods, which can be used for time variant as well as for time-invariant reliability analysis.

The FORM method is used as a fundamental procedure by a number of software products for the reliability analysis of structures and systems. The FORM method is also mentioned in EN 1990 as the background procedure applied to determine the design and combination values of basic variables, the partial factors, and various reduction factors applied in the partial factor method.

\section{$5 \quad$ Partial factor method and probabilistic design}

Consider an example of a steel member described by seven basic variables $\boldsymbol{X}$ (see equation (12)) given in Table 2: the random strength $\mathrm{f}_{\mathrm{y}}$, the deterministic design parameter $\mathrm{A}$ (variability of the design parameter $\mathrm{A}$ is taken into account by variability of $f_{\mathrm{y}}$ ), three random variables describing the actions: $G_{0}, Q_{0}$, and $W_{0}$ and two factors of model uncertainties $\theta_{R}$ (the resistance uncertainty) and $\theta_{E}$ (the load effect uncertainty) considered as random variables.

Table 2 indicates probabilistic models of basic variables for a time-invariant reliability analysis using Turkstra's rule (a combination of a 50-year maximum of leading action and an annual maximum of accompanying action).

The normalised strength and load effect are considered in the following numerical analysis assuming the characteristic strength $f_{\mathrm{k}}=1$ (without mentioning dimensions) and the load effect $E_{\mathrm{d}}=1$ for the load combination "A". Obviously, for the load combination "B" the load effect is smaller, thus $E_{\mathrm{d}}<1$. The partial factor methods for the load combination "A" then becomes trivial, from equation (10) the design parameter follows as $A=\gamma_{M}$. This simplification is accepted to enable easier comparison of both load combinations "A" and "B" of the partial factor method with more complicated probabilistic design.

Table 2. Probabilistic models of basic variables

\begin{tabular}{|lllccccc||}
\hline $\mathrm{N}^{\circ}$ & $\begin{array}{c}\text { Category of } \\
\text { variables }\end{array}$ & $\begin{array}{c}\text { Name of basic } \\
\text { variables }\end{array}$ & $\begin{array}{c}\text { Sym. } \\
X\end{array}$ & Dimension & $\begin{array}{c}\text { Distri- } \\
\text { bution }\end{array}$ & $\begin{array}{c}\text { Mean } \\
\mu_{X}\end{array}$ & $\begin{array}{c}\text { St.dev. } \\
\sigma_{X}\end{array}$ \\
\hline \hline 1 & Actions & Permanent & $G_{0}$ & $\mathrm{kN}$ & $\mathrm{N}$ & $G_{\mathrm{k}}$ & $0,1 \mu_{X}$ \\
2 & & Imposed - $50-\mathrm{yr}$ & $Q_{0}$ & $\mathrm{kN} / \mathrm{m}^{2}$ & $\mathrm{GU}$ & $0,6 Q_{\mathrm{k}}$ & $0,35 \mu_{X}$ \\
3 & & Wind - 1-yr & $\mathrm{W}_{0}$ & $\mathrm{kN} / \mathrm{m}^{2}$ & $\mathrm{GU}$ & $0,3 W_{\mathrm{k}}$ & $0,5 \mu_{X}$ \\
\hline 4 & Materials & Structural steel & $f_{\mathrm{y}}$ & $\mathrm{kN} / \mathrm{m}^{2}$ & $\mathrm{LN}$ & $f_{\mathrm{yk}}+2 \sigma_{X}$ & $0,08 \mu_{X}$ \\
\hline 5 & Geometric data & Design parameter & $A$ & $\mathrm{~m}^{2}$ & Det & $A_{\text {nom }}$ & 0 \\
\hline 6 & Model & Action effect fact. & $\theta_{E}$ & - & $\mathrm{LN}$ & 1,00 & $0,05 \mu_{X}$ \\
7 & Uncertainty & Steel bending & $\theta_{R}$ & - & $\mathrm{LN}$ & 1,00 & $0,05 \mu_{X}$ \\
\hline \hline
\end{tabular}

For a given design value of the load effect $E_{d}$ of the load combination "A" the characteristic values of individual actions $G_{\mathrm{k}}, Q_{\mathrm{k}}, W_{\mathrm{k}}$ must be expressed in terms of the load ratios $\chi$ and $\mathrm{k}$ using equation (6). Note that the load ratios $\chi$ and $\mathrm{k}$ are defined by equation (5). In the attached numerical example of a steel member the load ratios $\chi=0.4$ (a value typical for steel structures) and $k=0,0$ (lower reliability level than for $k>0,0$ ) are considered. If the 
load effect $E_{\mathrm{d}}=1$ for the load combination A it follows then from equation (6) that $G_{\mathrm{k}}=$ $0,426, Q_{\mathrm{k}}=0,284$ and $W_{\mathrm{k}}=0$.

A system of control and auxiliary functions for structural design developed using the language of technical computing MATLAB is applied for the partial factor methods and probabilistic design presented in Figure 1. The whole system contains a number of control and auxiliary functions intended for different design aspects and different structural materials. Note that the control function "SteelDes1" is applied for partial factor and probabilistic design of the considered steel member.

Figure 1 shows the design parameter $A$ determined by the partial factor method (assuming the load ratios $\chi=0.4$ and $k=0,0$, two partial factors $\gamma_{\mathrm{M}}=1,0$ and 1,1 and two combination rules "A" and "B") and variation of the reliability index $\beta$ and failure probability $p_{\mathrm{f}}$ with the design parameter $A$ (assuming three coefficients of variation of the steel strength $0,06,0,08,0,10$, which are supposed to also cover variation of geometric data generally affecting the design parameter $\mathrm{A}$ ).

The coefficient of variation of the steel strength seems to have an insignificant effect on the reliability level of the member. It appears that the required reliability level $\left(\beta=3,8, p_{\mathrm{f}}\right.$ $=7,2410^{-5}$ ) is satisfied if the design parameter is about $A=0,97$. If the partial factor method is used then the load combination rule "A" for the partial factor $\gamma_{\mathrm{M}}=1,00$ yields $\mathrm{A}=\gamma_{\mathrm{M}}=$ 1,00 , for the partial factor $\gamma_{\mathrm{M}}=1,10$ yields $A=\gamma_{\mathrm{M}}=1,10$; the load combination rule "B" for the partial factor $\gamma_{\mathrm{M}}=1,00$ yields $A=0,91$, for the partial factor $\gamma_{\mathrm{M}}=1,10$ yields $A=1,01$.

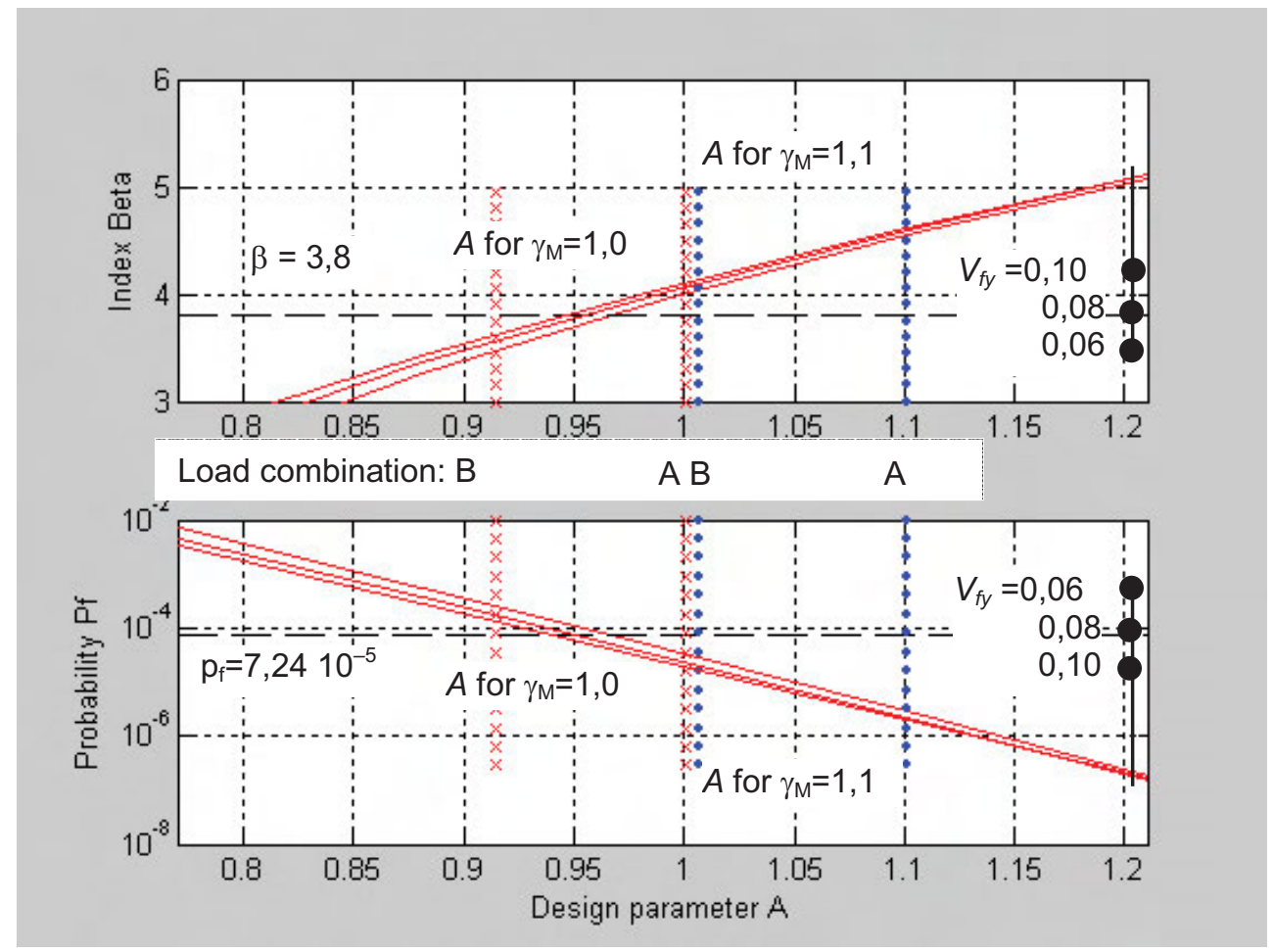

Figure 1. The design parameter $A$ determined by the partial factor method. 
It appears that the developed system of control and auxiliary functions may effectively help to implement the probabilistic design methods into common design practice.

Figure 2 shows the comparison of Turkstra's rule with the upper and lower bound of $p_{\mathrm{f}}$ determined by COMREL [24]. for the load ratios $\chi=0,4$ and $k=0,0$, and the coefficient of variation of steel strength 0,08 ; the lower and upper bounds of variable load $Q$ are determined assuming the mean as $0,2 Q_{\mathrm{k}}, w_{Q}=1,1$ and jump rate 0,2 ( 5 years).

It appears that the time-variant analysis provides reasonable bounds for the reliability index $\beta$ and the design values of the parameter A. Turkstra's rule seems to yield a conservative estimate of the reliability level and design value of the parameter A. Similar results are shown in Figure 3 for the load ratio $k=0,5$ when two variable actions $(Q$ and $W)$ are active.

Figure 3 shows the comparison of Turkstra's rule with the upper and lower bound of $p_{\mathrm{f}}$ determined by COMREL [24] for the load ratios $\chi=0.4$ and $\mathrm{k}=0,5$, and the coefficient of variation of steel strength 0,08 ; the lower and upper bounds are determined assuming for the variable load $Q$ the mean $0,2 Q_{\mathrm{k}}$, the coefficient of variation $w_{Q}=1,1$ and jump rate 0,2 ( 5 years), for the variable load $W$ the mean $0,5 \mathrm{~W}_{\mathrm{k}}$, the coefficient of variation $w_{W}=0.3$ and jump rate 10 (10 times a year) and duration coefficient 0.009 .

Figure 3 also indicates that if the total load effect is the same with an increasing number of variable actions the reliability level increases and the required design value of the parameter A decreases.

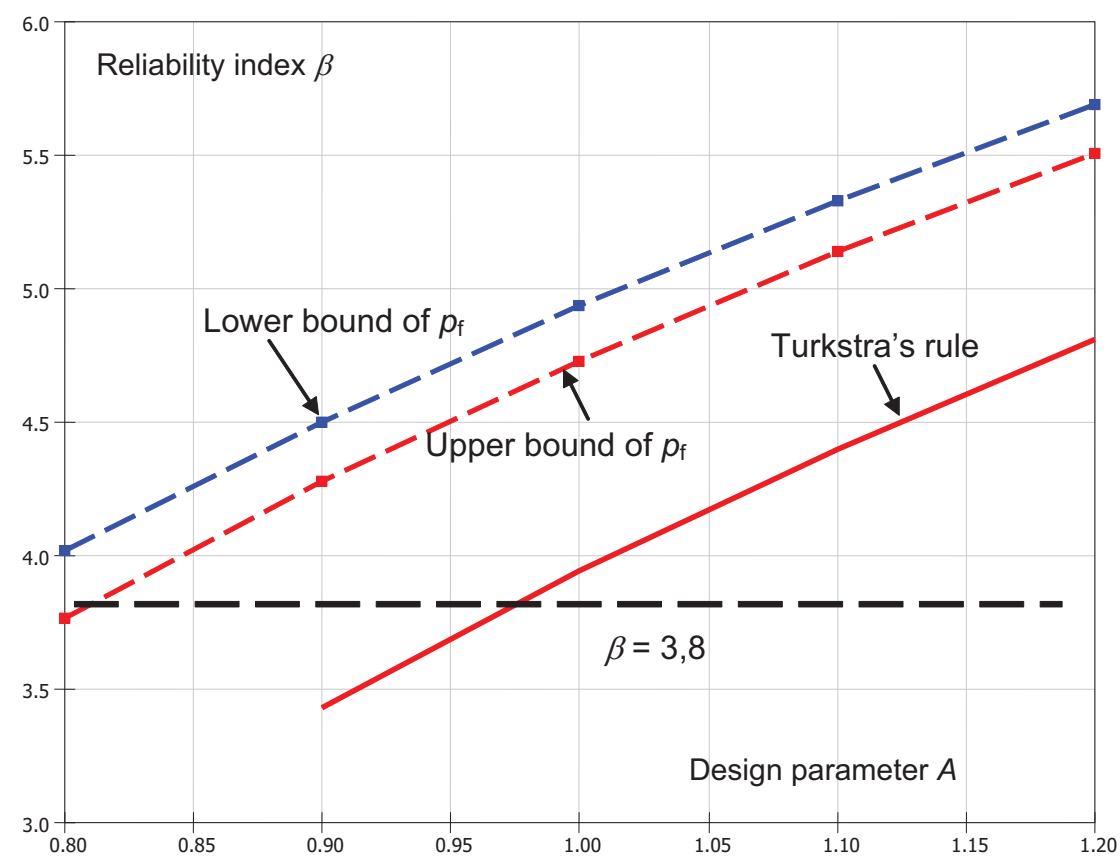

Figure 2. Comparison of Turkstra's rule $\chi=0,4$ and $k=0,0$, and the coefficient of variation of steel strength 0,08 . 


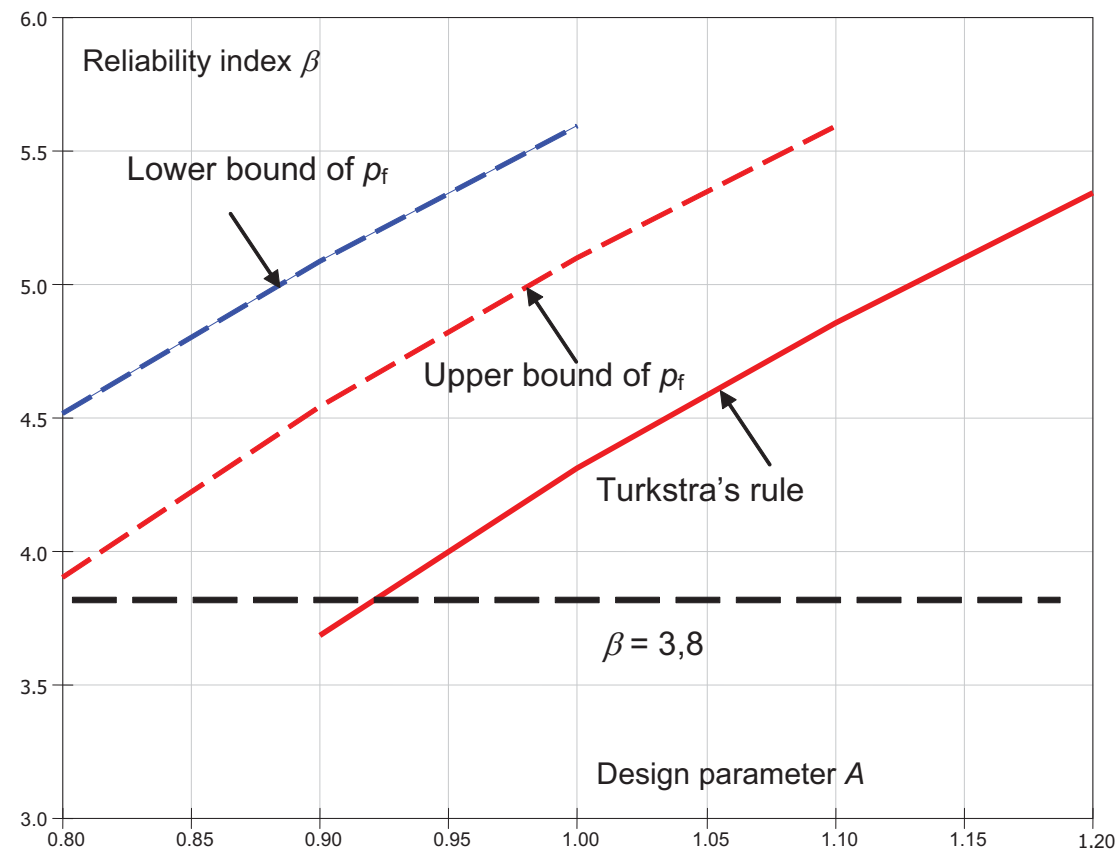

Figure 3. Comparison of Turkstra's rule $\chi=0,4$ and $k=0,5$, and the coefficient of variation of steel strength 0,08 .

The above findings confirm previous experience that the time-variant reliability analysis may lead to considerable economic effects. However, these effects are significantly dependent on particular conditions including the number of time-variant basic variables and their theoretical models. Practical application of time-variant reliability analysis should therefore include critical review of variable actions and theoretical models used for their description.

\section{Concluding remarks}

Figure 1 illustrates the relationship between the probabilistic design and the partial factor method of a steel member; assuming time-invariant analysis based on Turkstra's rule it shows the correspondence between the design parameter and the reliability index. Two main aspects of the partial factor method are considered in particular: alternative load combinations and the partial factor of material property (yield strength). Figure 1 indicates that both aspects have significant effects on the partial factor design and clearly illustrates the relationship between the considered alternatives and corresponding reliability level of the investigated structural member.

Considering a 50-year design life and the required reliability index $\beta=3,8$ it appears that the load combination "A" for the partial factor $\gamma_{M}=1,0$ and the load combination "B" for the partial factor $\gamma_{M}=1,1$ lead to almost the same satisfactory approximation of the probabilistic design for the required reliability index 3,8. The application of the load combination " $\mathrm{B}$ " together with the partial factor $\gamma_{M}=1,0$ seems to yield unsafe values of the design parameter (by about 5\% lower than the probabilistic design) while the application of 
the load combination "A" together with the partial factor $\gamma_{M}=1,1$ leads to slightly uneconomical design (by about $10 \%$ greater than the probabilistic design).

Thus, the following two almost equivalent alternatives leading to a satisfactory design may be recommended from the reliability point of view:

- the load combination "A" with the partial factr $\gamma_{M}=1,0$,

- the load combination "B" with the partial factor $\gamma_{M}=1,1$.

However, this recommendation is based on limited experience comprising only the presented simple example of a steel member. Further studies are needed to specify more definite conclusions; in particular, different load ratios $\chi, \mathrm{k}$ and different theoretical models of basic variables should be considered.

Figures 2 and 3 indicate the effect of more refined probabilistic design considering time-variant properties of variable actions described by the Ferry Borges-Castanheta model. It appears that the time-variant reliability analysis may lead to considerable economic effects in comparison with the time-invariant analysis based on Turstra's rule or with the deterministic design based on the partial factor method. However, these positive effects are significantly dependent on particular conditions including the number of time-variant basic variables (actions) and their theoretical models.

It appears that reliability methods may be effectively used for structural design and may bring considerable economic effects. Available software products including the developed system of MATLAB functions may contribute to an efficient implementation of probabilistic design into common design practice. 


\section{Annex 6: List of selected software tools supplementing the main text}

The software tools are intended for calculation procedures described in the main text. Three different software products are used to develop the tools: Excel, Mathcad and Matlab. The software tools are listed in the order of the relevant chapters of this textbook.

\section{Chapter 1}

\section{Excel sheets}

RCBeam.xls - Excel sheet intended for design of a reinforced concrete beam in accordance with various design methods.

\section{Mathcad sheets}

RCBeam.mcd - Mathcad sheet intended for design of a reinforced concrete beam in accordance with various design methods.

\section{Chapter 2}

\section{Excel sheet}

Update.xls - Excel sheet intended for updating of probabilities using Bayes' theorem.

\section{Mathcad sheets} theorem.

Update.mcd - Mathcad sheet intended for updating of probabilities using Bayes'

Update.prn - Mathcad file supplementing sheet Update.mcd with data.

\section{Chapter 3}

\section{Excel sheet}

DistUp.xls - Excel sheet intended for updating of distributions using Bayes' theorem.

\section{Mathcad sheets}

BetaDist.mcd - Mathcad sheet intended for specification of the parameters of Beta distribution.

GammaDist.med - Mathcad sheet intended for specification of the parameters of Gamma distribution.

LNDist.mcd - Mathcad sheet intended for specification of the parameters of Lognormal distribution.

Gumbelmax.mcd - Mathcad sheet intended for specification of the parameters of Gumbel distribution.

SampComb.mcd - Mathcad sheet intended for specification of the parameters of Beta distribution.

\section{Matlab sheets (see also Annex 7)}

Ndens.m - Matlab sheet for probability density function of a normal distribution.

Ndist.m - Matlab sheet for distribution function of a normal distribution.

Ndinv.m - Matlab sheet for the inverse distribution function of a normal distribution. 
Lndens.m - Matlab sheet for probability density function of a general three-parameter log-normal distribution.

Lndist.m - Matlab sheet for probability distribution function of a general threeparameter log-normal distribution.

Lndistinv.m - Matlab sheet for the inverse probability distribution function of a general three-parameter log-normal distribution.

\section{Chapter 4}

\section{Excel sheet}

Mod_est.xls - Excel sheet intended for estimation of resistance model fractiles using sample data and EN 1990 procedure.

\section{Mathcad sheets}

DisFract.mcd - Mathcad sheet intended for computation of fractiles for theoretical distribtutions.

SamFract.mcd - Mathcad sheet intended for estimation of fractiles using sample data.

Mod est.mcd - Mathcad sheet intended for estimation of resistance model fractiles using sample data and EN 1990 procedure.

BayesFract.mcd - Mathcad sheet intended for estimation of fractiles using sample data and Bayes' approach.

Cdata.prn - Mathcad file supplementing sheet Mod_est.med with data.

rData.prn - Mathcad file supplementing sheet Mod_est.mcd with data.

\section{Chapter 5}

\section{Excel sheets}

FORMRCB.xls - Excel sheet intended for FORM reliability analysis of a reinforced concrete beam.

FORM2.xls - Excel sheet intended for FORM reliability analysis of a fundamental case of two basic variables approximated by general three-parameter log-normal distribution.

FORM5.xls - Excel sheet intended for FORM reliability analysis of a case of five basic variables approximated by general three-parameter log-normal distribution.

FORM7.xls - Excel sheet intended for FORM reliability analysis of a case of seven basic variables approximated by general three-parameter log-normal distribution.

FORM7nonlin.xls - Excel sheet intended for FORM reliability analysis of a non-linear case of seven basic variables approximated by general three-parameter log-normal distribution.

\section{Mathcad sheets}

FORM_RCB.mcd - Mathcad sheet intended for FORM reliability analysis of a reinforced concrete beam.

FORM2.mcd - Mathcad sheet intended for FORM reliability analysis of a fundamental case of two basic variables approximated by general three-parameter log-normal distribution.

FORM5.mcd - Mathcad sheet intended for FORM reliability analysis of a case of five basic variables approximated by general three-parameter log-normal distribution.

FORM7.mcd - Mathcad sheet intended for FORM reliability analysis of a case of seven basic variables approximated by general three-parameter log-normal distribution. 
FORM7nonlin.mcd - Mathcad sheet intended for FORM reliability analysis of a nonlinear case of seven basic variables approximated by general three-parameter log-normal distribution.

Sim2.mcd - Mathcad sheet intended for reliability analysis of a fundamental case of two basic variables approximated by general three-parameter log-normal distribution using crude Monte Carlo method.

\section{Matlab sheet}

FORM2.m - Matlab sheet intended for FORM reliability analysis of a fundamental case of two basic variables approximated by general three-parameter log-normal distribution.

FORM5.m - Matlab sheet intended for FORM reliability analysis of a case of five basic variables approximated by general three-parameter log-normal distribution.

FORM7.m - Matlab sheet intended for FORM reliability analysis of a case of seven basic variables approximated by general three-parameter log-normal distribution.

Sim2.m - Matlab sheet intended for reliability analysis of a fundamental case of two basic variables approximated by general three-parameter log-normal distribution using crude Monte Carlo method.

\section{Chapter 6}

\section{Mathcad sheets}

PSI0.mcd - Mathcad sheet intended for computation of the factor $\psi_{0}$ assuming selected types of theoretical models.

PSI2.mcd - Mathcad sheet intended for computation of the factor $\psi_{2}$ assuming normal and Gumbel distribution.

\section{Chapter 8}

\section{Excel sheets}

GammaQ.xls - Excel sheet intended for computation of gamma Q based on input data including reliability index, sensitivity factor, reference intervals and time-sensitivity factor.

\section{Mathcad sheets}

GammaRGQ.mcd - Mathcad sheet intended for determination of partial factors for resistance variables, permanent and variable loads assuming various types of distributions.

\section{Matlab sheet}

GenerDes0.m - Matlab sheet for design of a generic structural member.

GenerDes1.m - Matlab sheet for design of a generic structural member (an alternative sheet to LoadEffect0.m - see Annex 7).

SteelDes1.m - Matlab sheet for design of a simple steel structural member.

CBeamDes1.m - Matlab sheet for design of a reinforced concrete beam.

CColumnDes1.m - Matlab sheet for design of a reinforced concrete column.

MasonDes 1.m - Matlab sheet for design of a simple masonry wall.

TimberDes1.m - Matlab sheet for design of a simple timber structural member.

LoadEffect0.m - Matlab sheet for specification of a load effect on a simple structural member.

LoadEffect1.m - Matlab sheet for specification of a load effect on a simple structural member (an alternative sheet to LoadEffect0.m - see Annex 7). 


\section{Annex 7: System of Matlab functions for probabilistic structural design}

\section{Introduction}

The developed system of MATLAB functions (small programs and interconnected functions) is primarily intended for deterministic (partial factor method) and probabilistic design of structural members. Load combinations are considered in accordance with the rules provided in EN 1990. Two combination rules are considered: the combination rule A corresponding to the expression (6.10) in EN 1990, and the combination rule B corresponding to twin expressions (6.10a) and (6.10b) in EN 1990. Resistance of selected structural members made of different materials is described taking into account the principles and rules of material oriented Eurocodes 1992 to 1996.

A typical system of MATLAB functions used for deterministic and probabilistic design of structural members is indicated in Figure 1.

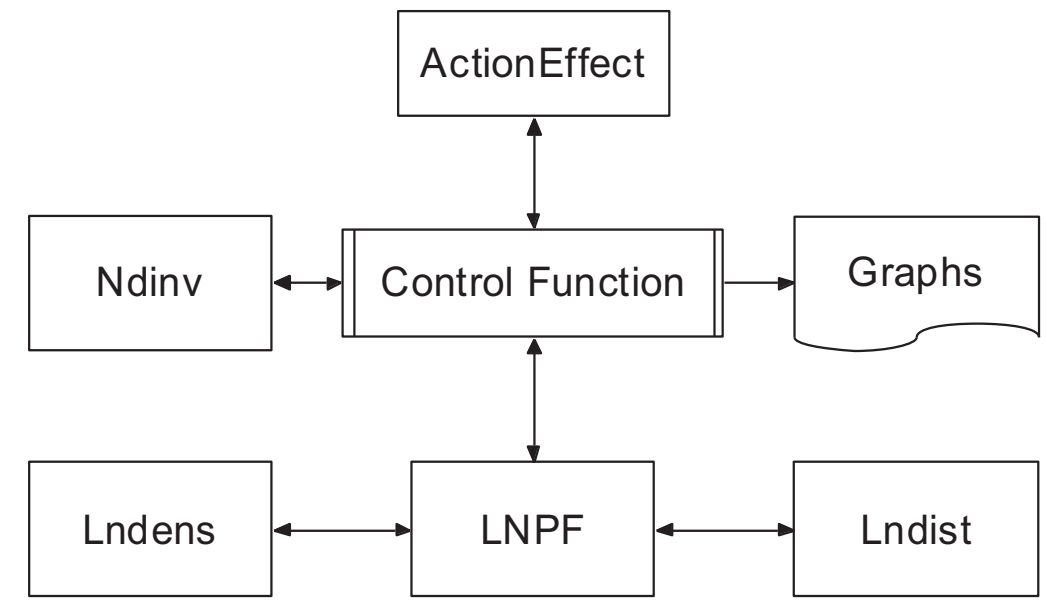

Figure 1. System of MATLAB functions used for design of structural members.

As indicated in Figure 1 the system of MATLAB functions for design of structural members consists of seven components: one control function, which specifies resistance parameters of a structural member and generates figures with the resulting graphs, and other auxiliary functions calculating appropriate load effects and failure probability (for different combination rules). Arrows in Figure 1 indicate interchange of data between individual functions of the system.

Obviously the central role of the system is provided by the control function (some control functions are still under construction), which defines parameters of a structural member and generates resulting graphs. In addition to the graphical results numerical outputs of selected quantities are also provided in the MATLAB command window. Failure probability is calculated by the function LNPF that further calls functions Lndens (calculating probability density function of three-parameter log-normal distribution) and Lndist (calculating distribution function of three-parameter log-normal distribution). 
A more detailed insight to the whole system may be obtained from the following list of control functions (programs) and other functions. The description of each function indicates its purpose and links to other functions. Additional information may also be found in the appropriate MATLAB files that include a number of comments and explanatory notes. Note that all the functions use some default parameters, which may, however, be adjusted taking into account particular design conditions of a given structural member.

\section{List of control functions}

Several alternative control functions (programs) have been developed to design simple structural members made of different materials. The control function generates a series of figures with graphs indicating variation of the reliability index and failure probability with a design parameter. These graphs together with information given in the Matlab command window enable decisions concerning the design parameter.

A concise description of each program is given in the following list of available programs. As indicated in Figure 1 any of these programs gradually calls a series of other MATLAB functions described below the list of the control functions. These functions calculate characteristics of load effects and failure probability using approximations of actual distributions by the three-parameter log-normal distribution for both the load effects and resistance.

\section{GenerDes0}

The control function GenerDes0(Ed,CHI,k,fk,gR) is the fundamental form of a program for design of a structural member. The arguments of this program are three parameters of actions: the total load effect Ed, load ratios CHI and k, the characteristic strength fk and the partial safety factor of the resistance gR. The characteristic action effects $\mathrm{Gk}, \mathrm{Qk}$ and Wk are calculated from Ed, CHI and $\mathrm{k}$. This form of the program is therefore useful to study the effect of various load ratios $\mathrm{CHI}$ and $\mathrm{k}$ on the resulting reliability level assuming a given load effect Ed.

The coefficient of variation wr of the resistance $\mathrm{R}$ is automatically chosen as three values: 0,$1 ; 0,15$ and 0,20 . A general structural member of the resistance $\mathrm{R}$ (without any reference to specific geometry and type of structural material) is described by the characteristic mean and the coefficient of variation wr (including model uncertainty).

The program GenerDes0(Ed,CHI,k,fk,gR) calls the functions

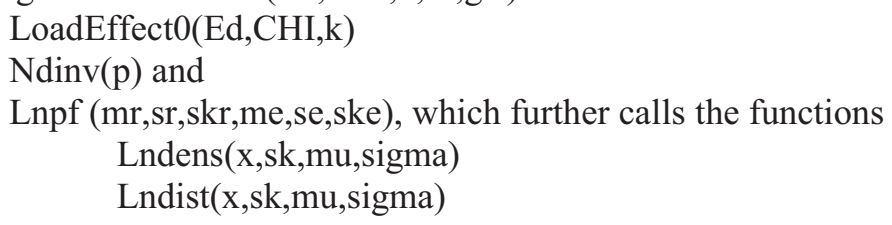

\section{GenerDes1}

The control function GenerDes1(Gk,Qk,Wk,fk,gR) is similar to the fundamental form GenerDes0. The program depends explicitly on three characteristic values of the load effects $\mathrm{Gk}, \mathrm{Qk}$ and $\mathrm{Wk}$, on the characteristic strength $\mathrm{fk}$ and on the partial safety factor of the resistance $\mathrm{gR}$. The design value EdA (for the combination $\mathrm{A}$ ) and $\mathrm{EdB}$ (for the combination B) of the load effect $\mathrm{E}$ is calculated from the characteristic actions Gk, Qk, Wk assuming the partial factors recommended in EN 1990. This control function is therefore useful for practical design procedures when the characteristic values of the load effects Gk, Qk and Wk are given. 
The coefficient of variation $\mathrm{wr}$ of the resistance $\mathrm{R}$ is automatically chosen as three values: $0,1,0,15$ and 0,20 . A general structural member of the resistance $\mathrm{R}$ (without any reference to specific geometry and material properties) is described by the normalised mean and the coefficient of variation wr (including model uncertainty).

The program GenerDes1(Gk,Qk,Wk,fk,gR) calls the functions

LoadEffect1(Gk,Qk,Wk)

$\operatorname{Ndinv}(p)$ and

Lnpf (mr,sr,skr,me,se,ske), which further calls the functions

Lndens(x,sk,mu,sigma)

Lndist(x,sk,mu,sigma)

\section{CBeamDes1 (exactly same as GenerDes1)}

The control function GenerDes1(Gk,Qk,Wk,fk,gR) is similar to the fundamental form GenerDes0. The program depends explicitly on three characteristic values of the load effects $\mathrm{Gk}, \mathrm{Qk}$ and $\mathrm{Wk}$, on the characteristic strength $\mathrm{fk}$ and on the partial safety factor of the resistance $\mathrm{gR}$. The design value EdA (for the combination $\mathrm{A}$ ) and $\mathrm{EdB}$ (for the combination B) of the load effect $\mathrm{E}$ is calculated from the characteristic actions Gk, Gk, Wk assuming the partial factors recommended in EN 1990. This form of the program is therefore useful for practical design procedures when the characteristic values of the load effects Gk, Gk and Wk are given.

The coefficient of variation wr of the resistance $\mathrm{R}$ is automatically chosen by three values: $0,1,0,15$ and 0,20 . A general structural member of the resistance $\mathrm{R}$ (without any reference to specific geometry and material properties) is described by the normalised mean and the coefficient of variation wr (including model uncertainty).

The program GenerDes1(Gk,Qk,Wk,fk,gR) calls the function

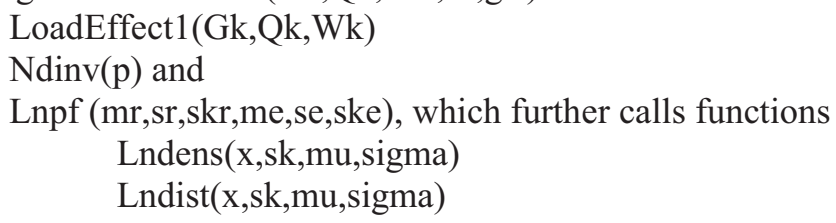

\section{SteelDes1}

The control function SteelDes1 is a modification of the function GenerDes1. The function SteelDes1(Gk,Qk,Wk,fk) depends explicitly on three characteristic values of the load effects $\mathrm{Gk}, \mathrm{Qk}$ and $\mathrm{Wk}$, on the characteristic strength fk of steel, and on the partial safety factor gm for steel. The design value EdA (for the combination A) and EdB (for the combination B) of the load effect $\mathrm{E}$ is calculated from the characteristic actions Gk, Qk, Wk assuming the partial factors recommended in EN 1990.

The function automatically chooses three coefficients of variation of steel strength wf $=0,06,0.08$ and 0.10 , and two different partial safety factors gm $(1,00$ and 1,10$)$ to show the effect of wf and gm on the resulting reliability of the deterministic design. The coefficient of variation of model uncertainty is assumed as $\mathrm{wKr}=0,05$.

The control function SteelDes1(Gk,Qk, Wk,fk) calls the functions

LoadEffect1(Gk,Qk,Wk)

$\operatorname{Ndinv}(\mathrm{p})$ and

Lnpf (mr,sr,skr,me,se,ske), which further calls functions

Lndens(x,sk,mu,sigma)

Lndist(x,sk,mu,sigma) 


\section{List of auxiliary functions}

\section{LoadEffect0}

Function LoadEffect0(EdA,CHI,k) calculates from the input arguments EdA, CHI and $\mathrm{k}$ the characteristic values of the load effects $\mathrm{Gk}, \mathrm{Qk}$ and Wk assuming the partial safety factors recommended in EN 1990. Using Gk, Qk and Wk the load effect EdB corresponding to the combination rule $\mathrm{B}$ (twin expressions (6.10a) and (6.10b)) is determined and the statistical characteristics me, se and ske of the load effect $\mathrm{E}$ are assessed.

The function is called by the command "LoadEffect(EdA,CHI,k)" and returns a vector [me,se,ske,EdB].

\section{LoadEffect1}

Function LoadEffect1(Gk,Qk,Wk) is used when the characteristic values of the load effects Gk, Qk and Wk are specified. From the input arguments Gk, Qk and Wk it calculates the load effects EdA corresponding to the combination rule A (expressions (6.10) in EN 1990 ) and EdB corresponding to the combination rule B (twin expressions (6.10a) and (6.10b) in EN 1990).

The function is called by the command "LoadEffect1(Gk,Qk,Wk)" and returns a vector [me,se,ske,EdA,EdB].

\section{LNPF}

Function LNPF is intended for determination of the failure probability using threeparameter log-normal distribution for both the load effect $\mathrm{E}$ and member resistance R. The function is called by the control function using command "Lnpf ( $\mathrm{mr}, \mathrm{sr}, \mathrm{skr}, \mathrm{me}, \mathrm{se}, \mathrm{ske})$ ". It returns the failure probability $\mathrm{p}$.

The function LNPF calls the functions

Lndens (x,sk,mu,sigma)

Lndist (x,sk,mu,sigma)

\section{Lndens}

Function Lndens is intended for calculation of the probability density function of three-parameter log-normal distribution. The function is called by the function LNPF using command "Lndens(ske,me,se)", and returns the value of probability density function of threeparameter log-normal distribution.

\section{Lndist}

Function Lndist is intended for calculation of the distribution function of threeparameter log-normal distribution. The function is called by the function LNPF using command "Lndist(skr,mr,sr)," and returns the value of the distribution function of threeparameter log-normal distribution.

\section{Ndinv}

Function Ndinv calculates the inverse distribution function of the normal distribution (giving the reliability index beta from failure probability). The function is called by the control function using command "Ndinv(p)" (commands "Ndinv(p,mu,sigma)" or "Ndinv(p,mu)" may be also used), and returns the inverse distribution function of the normal distribution. 


\section{Annex 8: Excel sheet FORM 7 and RORMRCB}

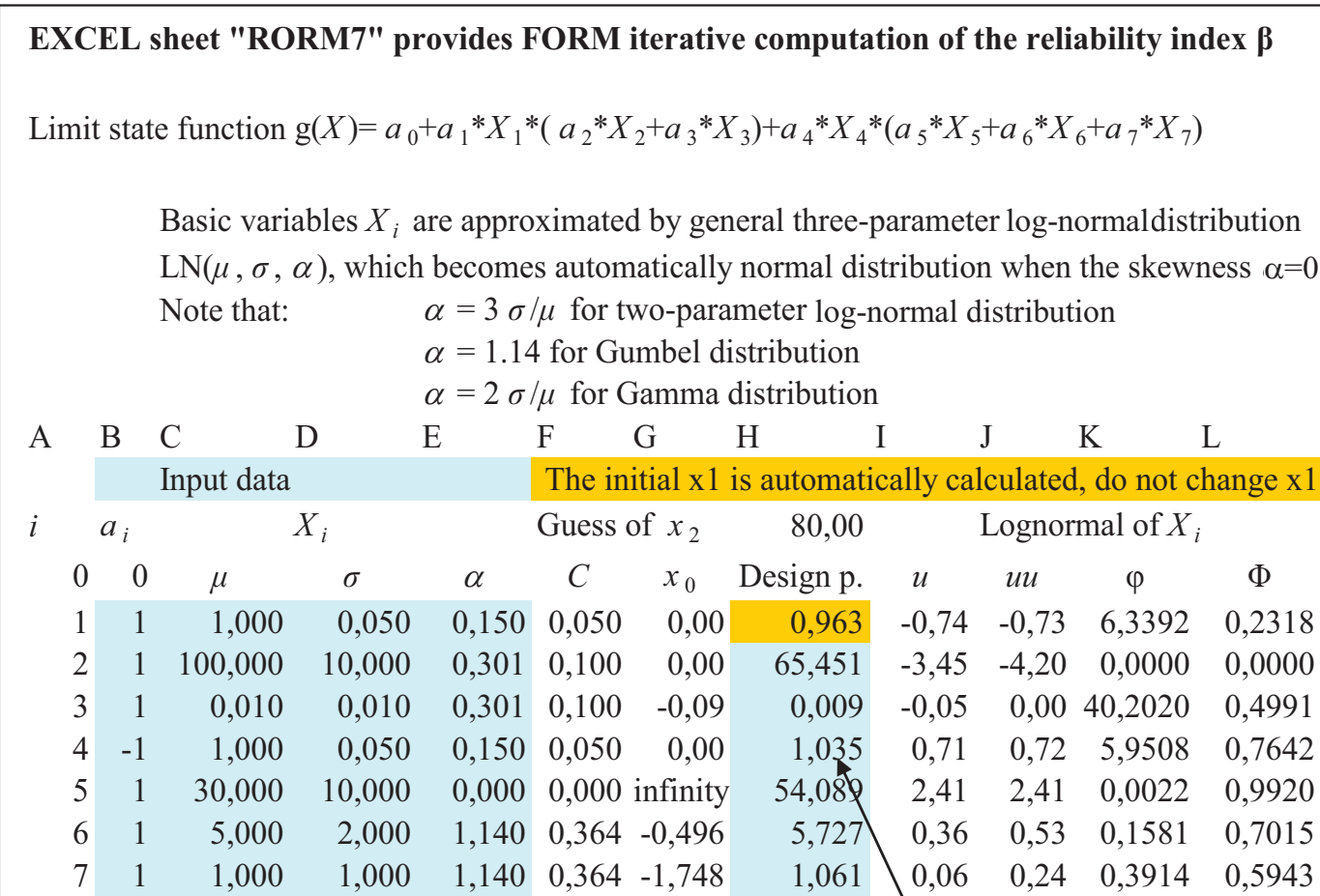

Requirement for the design point, $\alpha_{i}>0$ then $x_{0 i}<x_{i}$, when $\alpha_{i}<0$ then $x_{0 \mathrm{i}}>x_{i}$

Iteration of the FORM method - enter the new $x$ manually instead of the initial $x$ $a_{i} x_{i}$ Equivalent normal Derivatives Sensitivity New point Partial factors

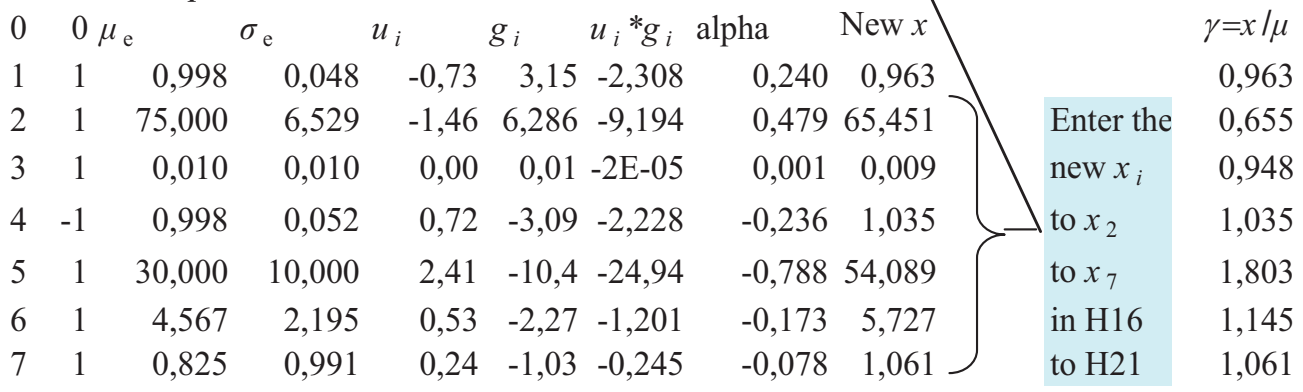
Sum $\quad 13,13-40,12 \quad 1,000$

\begin{tabular}{|c|c|c|}
\hline Number of iter. & $1 \beta$ in iter. $\mathrm{n}$ & 3,0551 Macro procedure \\
\hline Required acc. & \begin{tabular}{l|l}
0,001 & $\beta$ in iter. $\mathrm{n}-1$ \\
\end{tabular} & 5,5E-07 Accuracy reached \\
\hline
\end{tabular}

Probability of failure $p_{\mathrm{f}}=\Phi(-\beta)$ - command $=$ NORMDIST(-G34;0;1;1)

The whole iteration procedure may be performed manually following instruction given in the cells K27 to K32 or using Macro "ITERATION".

Note that the initial guess of variables $X_{2}$ to $X_{7}$ should be entered to the cells $\mathrm{H} 16$ to H21. 


\section{B. FORM method for determination of the reliability index beta}

Simplifier form of indexing is chosen, which is more suitable for counting:

$h=X_{1}, A_{s}=X_{2}, f_{y d}=X_{3}, c=X_{4}, b=X_{5}, f_{c d}=X_{6}, K=X_{7}, G=X_{8}, Q=X_{9}, W=X_{10}$

3. Input parameters for basic variables $\{X\}=\left\{X_{1}, X_{2} \ldots X_{10}\right\}$ and the coefficients

$a_{7}, a_{8}, a_{9}$ and $a_{10}$ may be written in following re-indexed limit state function:

$$
g(X)=X_{1}{ }^{*} X_{2}{ }^{*} X_{3}-X_{4}{ }^{*} X_{2}{ }^{*} X_{3}-X_{2}{ }^{2} X_{3}{ }^{2} /\left(2{ }^{*} X_{5}{ }^{*} X_{6}\right)+a_{7}{ }^{*} X_{7}{ }^{*}\left(a_{8}{ }^{*} X_{8}+a_{9}{ }^{*} X_{9}+a_{10}{ }^{*} X_{10}\right)
$$

\begin{tabular}{|c|c|c|c|c|c|c|}
\hline \multicolumn{2}{|c|}{ Input Variables } & i & a & $\mu$ & $\sigma$ & $\alpha$ \\
\hline \multirow{6}{*}{ 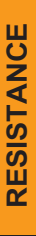 } & Height of cross-section $\mathrm{h}$ & $x_{01}$ & - & 0,3 & 0,01 & 0 \\
\hline & Area of reinforcement $A_{s}$ & $x_{02}$ & - & 0,0004 & 0,00002 & 0,15 \\
\hline & Yield strength of reinforcement $f_{y}$ & $x_{03}$ & - & 560000 & 30000 & 0,15 \\
\hline & Cover c & $x_{04}$ & - & 0,03 & 0,005 & 0,5 \\
\hline & Width of cross-section b & $x_{05}$ & - & 0,2 & 0,005 & 0 \\
\hline & Concrete strength $f_{c}$ & $x_{06}$ & - & 30000 & 5000 & 0,5 \\
\hline \multirow{4}{*}{ 足 } & Model uncertainty $\mathrm{K}$ & $x_{07}$ & -1 & 1 & 0,05 & 0,15 \\
\hline & Permanent load G & $x_{08}$ & 1 & 18 & 1,8 & 0 \\
\hline & Variable load Q & $x_{09}$ & 1 & 10 & 2 & 1,14 \\
\hline & Variable load W & $x_{10}$ & 1 & 2 & 1 & 1,14 \\
\hline
\end{tabular}

The check of the initial guess values

$$
\begin{aligned}
& x_{1}:=\mu_{i} \quad x_{1}:=\frac{x_{4} \cdot x_{2} \cdot x_{3}+\frac{\left(x_{2}\right)^{2} \cdot\left(x_{3}\right)^{2}}{2 \cdot x_{5} \cdot x_{6}}-a_{7} \cdot x_{7} \cdot\left(a_{8} \cdot x_{8}+a_{9} \cdot x_{9}+a_{10} \cdot x_{10}\right)}{x_{2} \cdot x_{3}} \quad \begin{array}{ll}
x_{1}= & 0,1826
\end{array}
\end{aligned}
$$

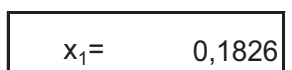

4. FORM iteration procedure:

Symbolic iteration procedure

The initial guess

value of $X_{1}$ is

calculated from $g(X)=0$

Number of iterations $\mathrm{n}$

Equivalent normal

distributions of

the basic variables $X$.

Standardised variables

Partial derivatives of $g(X)$ :

$$
\beta_{n}:=\mid \begin{aligned}
& x \leftarrow \mu \\
& x_{1} \leftarrow \frac{x_{4} \cdot x_{2} \cdot x_{3}+\frac{\left(x_{2}\right)^{2} \cdot\left(x_{3}\right)^{2}}{2 \cdot x_{5} \cdot x_{6}}-a_{7} \cdot x_{7} \cdot\left(a_{8} \cdot x_{8}+a_{9} \cdot x_{9}+a_{10} \cdot x_{10}\right)}{x_{2} \cdot x_{3}} \\
& \text { for } j \in 1 \ldots n
\end{aligned}
$$

$$
\left\{\begin{array}{l}
\int_{i} \leftarrow \frac{\operatorname{dnorm}\left(\operatorname{qnorm}\left(\Phi\left(\mathrm{x}_{\mathrm{i}}, \mu_{\mathrm{i}}, \sigma_{\mathrm{i}}, \alpha_{\mathrm{i}}\right), 0,1\right), 0,1\right)}{\phi\left(\mathrm{x}_{\mathrm{i}}, \mu_{\mathrm{i}}, \sigma_{\mathrm{i}}, \alpha_{\mathrm{i}}\right)} \\
\mu_{\mathrm{i}} \leftarrow \mathrm{x}_{\mathrm{i}}-\sigma_{\mathrm{i}} \cdot \mathrm{qnorm}\left(\Phi\left(\mathrm{x}_{\mathrm{i}}, \mu_{\mathrm{i}}, \sigma_{\mathrm{i}}, \alpha_{\mathrm{i}}\right), 0,1\right) \\
\mathrm{u}_{\mathrm{i}} \leftarrow \frac{\mathrm{x}_{\mathrm{i}}-\mu \mathrm{e}_{\mathrm{i}}}{\sigma \mathrm{e}_{\mathrm{i}}} \\
\mathrm{g}_{1} \leftarrow \mathrm{x}_{2} \cdot \mathrm{x}_{3} \cdot \sigma \mathrm{e}_{1} \\
\mathrm{~g}_{2} \leftarrow\left[\mathrm{x}_{1} \cdot \mathrm{x}_{3}-\mathrm{x}_{4} \cdot \mathrm{x}_{3}-\frac{\mathrm{x}_{2} \cdot\left(\mathrm{x}_{3}\right)^{2}}{\left.2 \cdot \mathrm{x}_{5} \cdot \mathrm{x}_{6}\right] \cdot \sigma \mathrm{e}_{2}}\right. \\
\mathrm{g}_{3} \leftarrow\left[\mathrm{x}_{1} \cdot \mathrm{x}_{2}-\mathrm{x}_{2} \mathrm{x}_{4}-\frac{\left[\left(\mathrm{x}_{2}\right)^{2} \mathrm{x}_{3}\right]}{\mathrm{x}_{5} \mathrm{x}_{6}}\right] \cdot \sigma \mathrm{e}_{3} \\
\mathrm{~g}_{4} \leftarrow-\mathrm{x}_{2} \cdot \mathrm{x}_{3} \cdot \sigma \mathrm{e}_{4} \\
\mathrm{~g}_{5} \leftarrow \frac{\left(\mathrm{x}_{2}\right)^{2} \cdot\left(\mathrm{x}_{3}\right)^{2}}{2 \cdot\left(\mathrm{x}_{5}\right)^{2} \cdot \mathrm{x}_{6}} \cdot \sigma \mathrm{e}_{5}
\end{array}\right.
$$




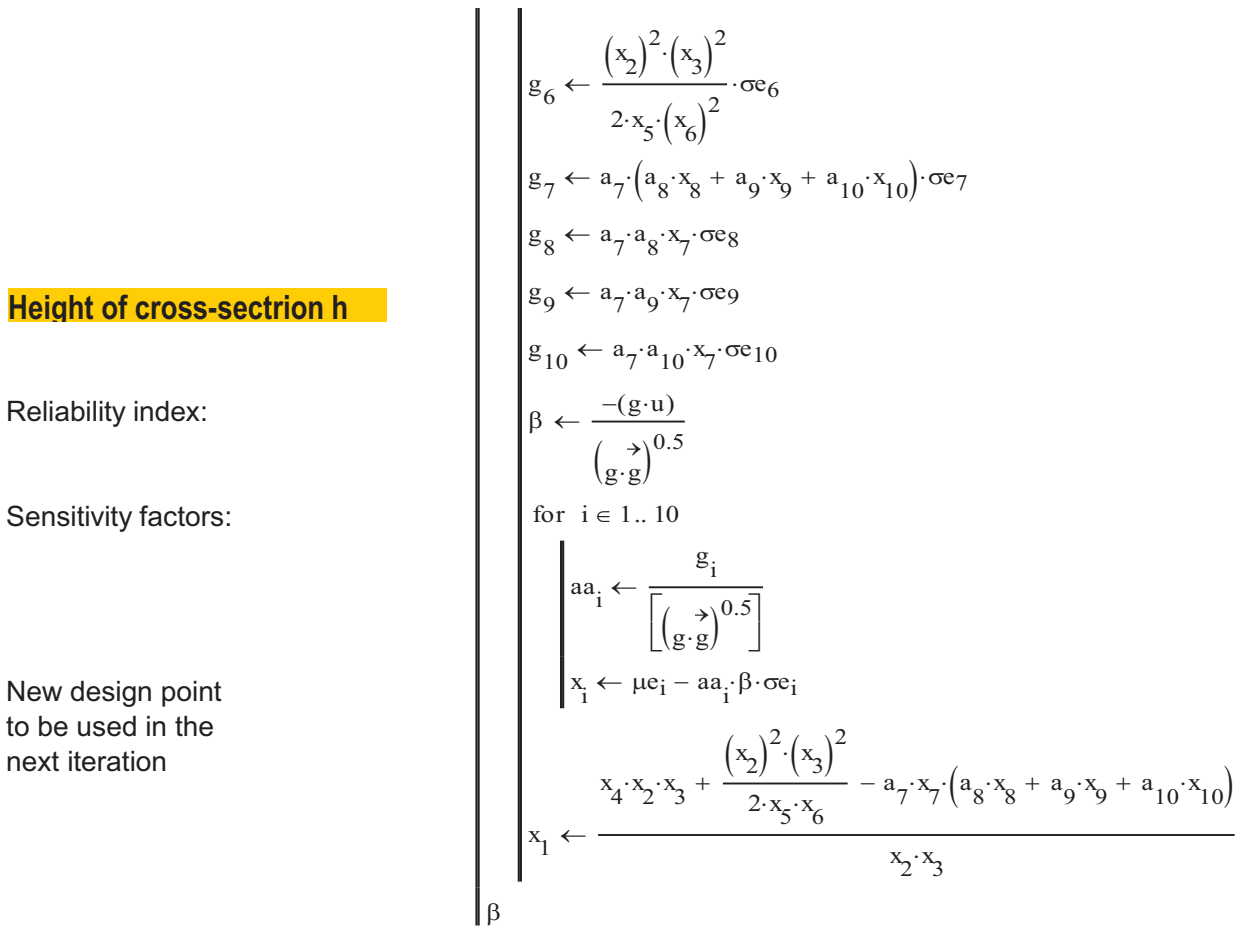

\section{FORM Iteration:}

Number of iteration:

value

\begin{tabular}{|c|c|c|c|c|c|c|c|c|c|c|}
\hline$X i$ & $x_{i}$ & $\begin{array}{c}\text { nominato } \\
r \sigma_{\mathrm{ei}}\end{array}$ & $\begin{array}{c}\text { denominat } \\
\text { or } \sigma_{\mathrm{ei}}\end{array}$ & $\sigma_{\mathrm{ei}}$ & $\mu_{\mathrm{ei}}$ & $\mu_{\mathrm{i}}$ & $g_{i}$ & $a a_{i}$ & $\mathrm{X}_{\mathrm{i}+1}$ & $\gamma=\mathrm{x} / \mu$ \\
\hline $\bar{h}$ & 0,2896 & 0,2331 & 23,3088 & $\overline{0,0100}$ & 0,3000 & $-1,0367$ & 1,9646 & 0,2347 & 0,2896 & 0,9654 \\
\hline As & 0,0004 & 0,1845 & 9833,1 & 0,0000 & 0,0004 & $-1,2419$ & 2,3535 & 0,2811 & 0,0004 & 0,9387 \\
\hline fy & 523262 & 0,1851 & 0,0000 & 28147,1 & 558149,9 & $-1,2395$ & 2,3489 & 0,2806 & 523266 & 0,9344 \\
\hline C & 0,0325 & 0,3417 & 63,6473 & 0,0054 & 0,0295 & 0,5566 & $-1,0547$ & $-0,1260$ & 0,0325 & 1,0817 \\
\hline$b$ & 0,1998 & 0,3985 & 79,7066 & 0,0050 & 0,2000 & $-0,0453$ & 0,0859 & 0,0103 & 0,1998 & 0,9989 \\
\hline fcd & 28161,6 & 0,3814 & 0,0001 & 4664,8 & 29560,4 & $-0,2998$ & 0,5682 & 0,0679 & 28161,8 & 0,9387 \\
\hline $\mathrm{K}$ & 1,0627 & 0,1845 & 3,4751 & 0,0531 & 0,9968 & 1,2418 & $-2,3533$ & $-0,2811$ & 1,0627 & 1,0627 \\
\hline G & 19,8171 & 0,2397 & 0,1332 & 1,8000 & 18,0000 & 1,0095 & $-1,9128$ & $-0,2285$ & 19,8167 & 1,1009 \\
\hline Q & 22,0129 & 0,0010 & 0,0002 & 6,1748 & 0,6361 & 3,4619 & $-6,5619$ & $-0,7838$ & 22,0151 & 2,2015 \\
\hline W & 2,4884 & 0,3250 & 0,2848 & 1,1413 & 1,7577 & 0,6403 & $-1,2129$ & $-0,1449$ & 2,4881 & 1,244 \\
\hline
\end{tabular}

Reliability index $\beta$ :

$\beta_{\mathrm{i}}=\quad 4,42$

Probability of failure $p f: \quad \quad p f:=\operatorname{normdist}\left(-\beta_{\mathrm{i}}, 0,1,1\right)$

$p f=\quad 5,00 E-06$




\section{Annex 9: Mathcad sheet FORM 7}

MATHCAD sheet "FORM7" for calculation of the reliability inde $\beta$ and failure probability assuming a non-linear limit state function $g(X)=a 0+a 1^{*} X 1\left(a 2^{*} X 2+a 3^{*} X 3\right)+a 4^{*} X 4\left(a 5^{*} X 5+a 6 X 6+a 7 X 7\right)$ and general three-parameter log-normal distribution $\operatorname{LN}(\sigma, \alpha)$ of basic variables $X 1, X 2, X 3, X 4, X 5, X 6$ and $X 7$

A General three-parameter log-normal distribution for any $\alpha$

1. Parameter $\mathrm{C}$ and skewness $\alpha$ :

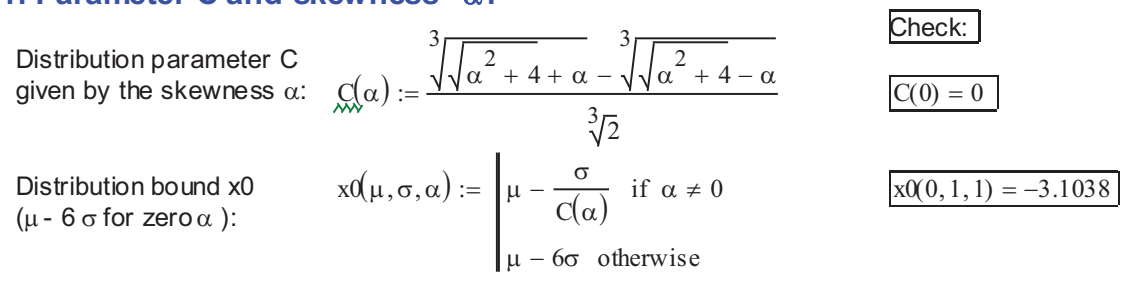

2. Probability density $\phi$ and distribution function $\Phi$ (for any $\alpha$ ):

Standardised variable: $\quad \mathrm{u}(\mathrm{x}, \mu, \sigma):=\frac{\mathrm{x}-\mu}{\sigma} \quad$ Transformed standardised variable:

$$
\mathrm{uu}(\mathrm{x}, \mu, \sigma, \alpha):=\mid \begin{aligned}
& \ln \left(\left|\mathrm{u}(\mathrm{x}, \mu, \sigma)+\frac{1}{\mathrm{C}(\alpha)}\right|\right)+\ln \left(|\mathrm{C}(\alpha)| \cdot \sqrt{1+\mathrm{C}(\alpha)^{2}}\right) \\
& \operatorname{sign}(\alpha) \cdot \sqrt{\ln \left(1+\mathrm{C}(\alpha)^{2}\right)}
\end{aligned} \text { if } \alpha \neq 0
$$

Density probability function:

$$
\begin{array}{|l|l|}
\phi(\mathrm{x}, \mu, \sigma, \alpha):=\mid \frac{\mathrm{dnorm}(\mathrm{uu}(\mathrm{x}, \mu, \sigma, \alpha), 0,1)}{\sigma \cdot\left|\mathrm{u}(\mathrm{x}, \mu, \sigma)+\frac{1}{\mathrm{C}(\alpha)}\right| \cdot \sqrt{\ln \left(1+\mathrm{C}(\alpha)^{2}\right)}} \text { if } \alpha \neq 0 & \phi(50,50,1,0)=0.3989 \\
\frac{\mathrm{dnorm}(\mathrm{uu}(\mathrm{x}, \mu, \sigma, \alpha), 0,1)}{\sigma} \text { otherwise } & \Phi(100,100,10,0)=0.5
\end{array}
$$

B FORM method for determination of the reliability index $\beta$ and probability pf Coefficients a0, a1, a2, a3, a4, a5, a6 and a7 of the limit state functions and Input parameters for basic variables $\{X\}=\{X 1, X 2, X 3, X 4, X 5, X 6$ and $X 7\}$

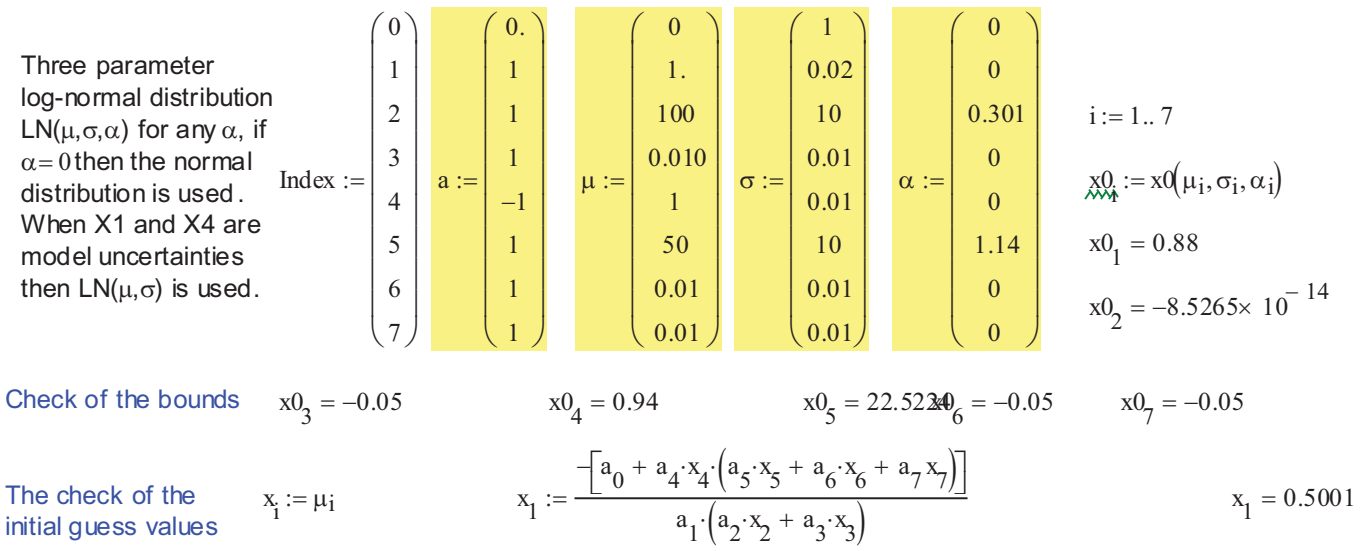


4. FORM iteration procedure: Probability of failure pfis determined from the reliability index $\beta$

Number of iterations $\quad \mathrm{n}:=1 . .5$

The initial guess values of $X$

The value $x 2$

is calculated

from $g(X)=0$

\section{Equivalent \\ normal \\ distributions of \\ the basic \\ variables $X$}

Standardised variables

Derivatives of $g(X)$ :

Reliability index:

Sensitivity factors:

New design point to be used in the next iteration, go back to the section 5 and use this data in a new run

Iteration of the reliability index $\beta$

Probability of failure pf

$$
\begin{aligned}
& \beta_{\mathrm{n}}:=\mid \begin{array}{l}
\mathrm{x} \leftarrow \mu \\
\mathrm{x}_{1} \leftarrow \frac{-\left[\mathrm{a}_{0}+\mathrm{a}_{4} \cdot \mathrm{x}_{4} \cdot\left(\mathrm{a}_{5} \cdot \mathrm{x}_{5}+\mathrm{a}_{6} \cdot \mathrm{x}_{6}+\mathrm{a}_{7} \mathrm{x}_{7}\right)\right]}{\mathrm{a}_{1} \cdot\left(\mathrm{a}_{2} \cdot \mathrm{x}_{2}+\mathrm{a}_{3} \cdot \mathrm{x}_{3}\right)} \\
\text { for } \mathrm{j} \in 1 . . \mathrm{n}
\end{array} \\
& \text { for } i \in 1 . .7 \\
& \sigma_{\mathrm{i}} \leftarrow \frac{\operatorname{dnorm}\left(\operatorname{qnorm}\left(\Phi\left(\mathrm{x}_{\mathrm{i}}, \mu_{\mathrm{i}}, \sigma_{\mathrm{i}}, \alpha_{\mathrm{i}}\right), 0,1\right), 0,1\right)}{\phi\left(\mathrm{x}_{\mathrm{i}}, \mu_{\mathrm{i}}, \sigma_{\mathrm{i}}, \alpha_{\mathrm{i}}\right)} \\
& \mu \mathrm{e}_{\mathrm{i}} \leftarrow \mathrm{x}_{\mathrm{i}}-\sigma \mathrm{e}_{\mathrm{i}} \cdot \operatorname{qnorm}\left(\Phi\left(\mathrm{x}_{\mathrm{i}}, \mu_{\mathrm{i}}, \sigma_{\mathrm{i}}, \alpha_{\mathrm{i}}\right), 0,1\right) \\
& \mathrm{u}_{\mathrm{i}} \leftarrow \frac{\mathrm{x}_{\mathrm{i}}-\mu \mathrm{e}_{\mathrm{i}}}{\sigma \mathrm{e}_{\mathrm{i}}} \\
& \mathrm{g}_{1} \leftarrow \mathrm{a}_{1} \cdot\left(\mathrm{a}_{2} \cdot \mathrm{x}_{2}+\mathrm{a}_{3} \cdot \mathrm{x}_{3}\right) \sigma \mathrm{e}_{1} \\
& \mathrm{~g}_{2} \leftarrow \mathrm{a}_{1} \cdot \mathrm{a}_{2} \cdot \mathrm{x}_{1} \cdot \sigma \mathrm{e}_{2} \\
& \mathrm{~g}_{3} \leftarrow \mathrm{a}_{1} \cdot \mathrm{a}_{3} \cdot \mathrm{x}_{1} \cdot \sigma \mathrm{e}_{3} \\
& \mathrm{~g}_{4} \leftarrow \mathrm{a}_{4} \cdot\left(\mathrm{a}_{5} \cdot \mathrm{x}_{5}+\mathrm{a}_{6} \cdot \mathrm{x}_{6}\right) \cdot \sigma \mathrm{e}_{4} \\
& \mathrm{~g}_{5} \leftarrow \mathrm{a}_{4} \cdot \mathrm{a}_{5} \cdot \mathrm{x}_{4} \cdot \sigma \mathrm{e}_{5} \\
& \mathrm{~g}_{6} \leftarrow \mathrm{a}_{4} \cdot \mathrm{a}_{6} \cdot \mathrm{x}_{4} \cdot \sigma \mathrm{e}_{6} \\
& \mathrm{~g}_{7} \leftarrow \mathrm{a}_{4} \cdot \mathrm{a}_{7} \cdot \mathrm{x}_{4} \cdot \sigma \mathrm{e} 7 \\
& \beta \leftarrow \frac{-(\mathrm{g} \cdot \mathrm{u})}{\left(\begin{array}{r}
\mathrm{g} \cdot \mathrm{g}
\end{array}\right)^{0.5}} \\
& \text { for } i \in 1 . .7 \\
& \mid \begin{array}{l}
\mid \begin{array}{l}
\mathrm{aa}_{\mathrm{i}} \leftarrow \frac{\mathrm{g}_{\mathrm{i}}}{\left[(\mathrm{g} \cdot \mathrm{g})^{0.5}\right]} \\
\mathrm{x}_{1} \leftarrow \mu \mathrm{e}_{\mathrm{i}}-\mathrm{aa}_{\mathrm{i}} \cdot \beta \cdot \sigma \mathrm{e}_{\mathrm{i}}
\end{array} \\
\mathrm{x}_{1} \leftarrow \frac{-\left[\mathrm{a}_{0}+\mathrm{a}_{4} \cdot \mathrm{x}_{4} \cdot\left(\mathrm{a}_{5} \cdot \mathrm{x}_{5}+\mathrm{a}_{6} \cdot \mathrm{x}_{6}+\mathrm{a}_{7} \mathrm{x}_{7}\right)\right]}{\mathrm{a}_{1} \cdot\left(\mathrm{a}_{2} \cdot \mathrm{x}_{2}+\mathrm{a}_{3} \cdot \mathrm{x}_{3}\right)}
\end{array}
\end{aligned}
$$

$$
\beta=\left(\begin{array}{c}
0 \\
2.9022 \\
2.9303 \\
2.9292 \\
2.929 \\
2.9289
\end{array}\right)
$$

$$
\text { pf := pnorm }\left(-\beta_{5}, 0,1\right)
$$$$
\mathrm{pf}=1.7006 \times 10^{-3}
$$ 
Annex 10: Matlab sheet FORM 7

function pf $=$ FORM7

$\%$ DESCRIPTION, 28.02.2005

$\%$ FORM7a evaluates the the probability of failure pf considering the limit state function

$\%$

$\% \quad \mathrm{~g}(\mathrm{X})=\mathrm{A} 0+\mathrm{A} 1 * \mathrm{X} 1 *(\mathrm{~A} 2 * \mathrm{X} 2+\mathrm{A} 3 * \mathrm{X} 3)+\mathrm{A} 4 * \mathrm{X} 4 *(\mathrm{~A} 5 * \mathrm{X} 5+\mathrm{A} 6 * \mathrm{X} 6+\mathrm{A} 7+\mathrm{A} 7)$

$\%$

$\%$ FUNCTIONS USED

$\% \quad$ LNDENS(x,ske,me,se), LNDIST(x,skr,mr,sr), NDENS(x), NDIST(x) and NDINV(p)

$\%$ INPUT

$\% \quad$ Input data (except A0) are loaded from the files A.dat (coefficients A), and X.dat (parameters

$\%$ of the basic variables $\mathrm{X}$ ). All the basic variables are characterised by the mean $\mathrm{m}$, standard deviation $s$

$\%$ and skewness sk (arbitrary). The FORM procedure approximates the basic variables by general

$\% \quad \log$-normal distribution $\mathrm{LN}(\mathrm{m}, \mathrm{s}, \mathrm{sk})$, including normal distribution (for sk=0).

$\%$

$\%$ OUPUT

$\%$ val : failure probability pf

$\%$ VERSION

$\% \quad$ MH, Czech Technical University in Prague, Klokner Institute, 28.2.2005

$\%$ Initialization

$\%$ \%loading external data files

load A.dat, load X.dat, \% The matrix $X$ can also be defined in the command window $\mathrm{A} 0=0 ; \%$ additive constant (not included in the data file A.dot), default value $\mathrm{A} 0=0$.

When $\mathrm{A0}$ is different from $\mathbf{0}$,

$\%$ numerical problems may arise. Then a new alternative initial point (for example modifying resistance) may be chosen.

for $i=1: 1: 7$;

$\mathbf{x}(i)=X(i, 1) ; \%$ Initial guess value of basic variables end

$x(1)=-(A 0+A(4) * x(4) *(A(5) * x(5)+A(6) * x(6)+A(7) * x(7))) /(A(1) *(A(2) * x(2)+A(3) * x(3)))$ $\%$ Initial guess value of $x 1$

$\%$ FORM iterations

acc $=0.001 ;$ delta $=1 ;$ betap $=0 ; j=0 ; \%$ required accuracy (may be modified if required)initial iteration parameters

while delta $>$ acc $\%$ for $j=1: 1: 5 \%$ Iteration loop for a given accuracy (5 cycles are usually sufficient for acc $=\mathbf{0 . 0 0 1}$ )

$\mathbf{j}=\mathbf{j}+\mathbf{1} ; \%$ The indicator of the number of cycles

for $i=1: 1: 7$; \% Loop for transformation of original distributions to equivalent normal ditributions

se(i)=NDENS(norminv $(\operatorname{LNDIST}(x(\mathbf{i}), X(i, 1), X(i, 2), X(i, 3)))) / \operatorname{LNDENS}(x(\mathbf{i}), X(i, 1), X(i, 2), X(i$ ,3));

$\operatorname{me}(i)=x(i)-\operatorname{se}(i) * n o r m i n v(\operatorname{LNDIST}(x(i), X(i, 1), X(i, 2), X(i, 3)))$;

$u(i)=(x(i)-m e(i)) / s e(i) ; \%$ Standardized variables 


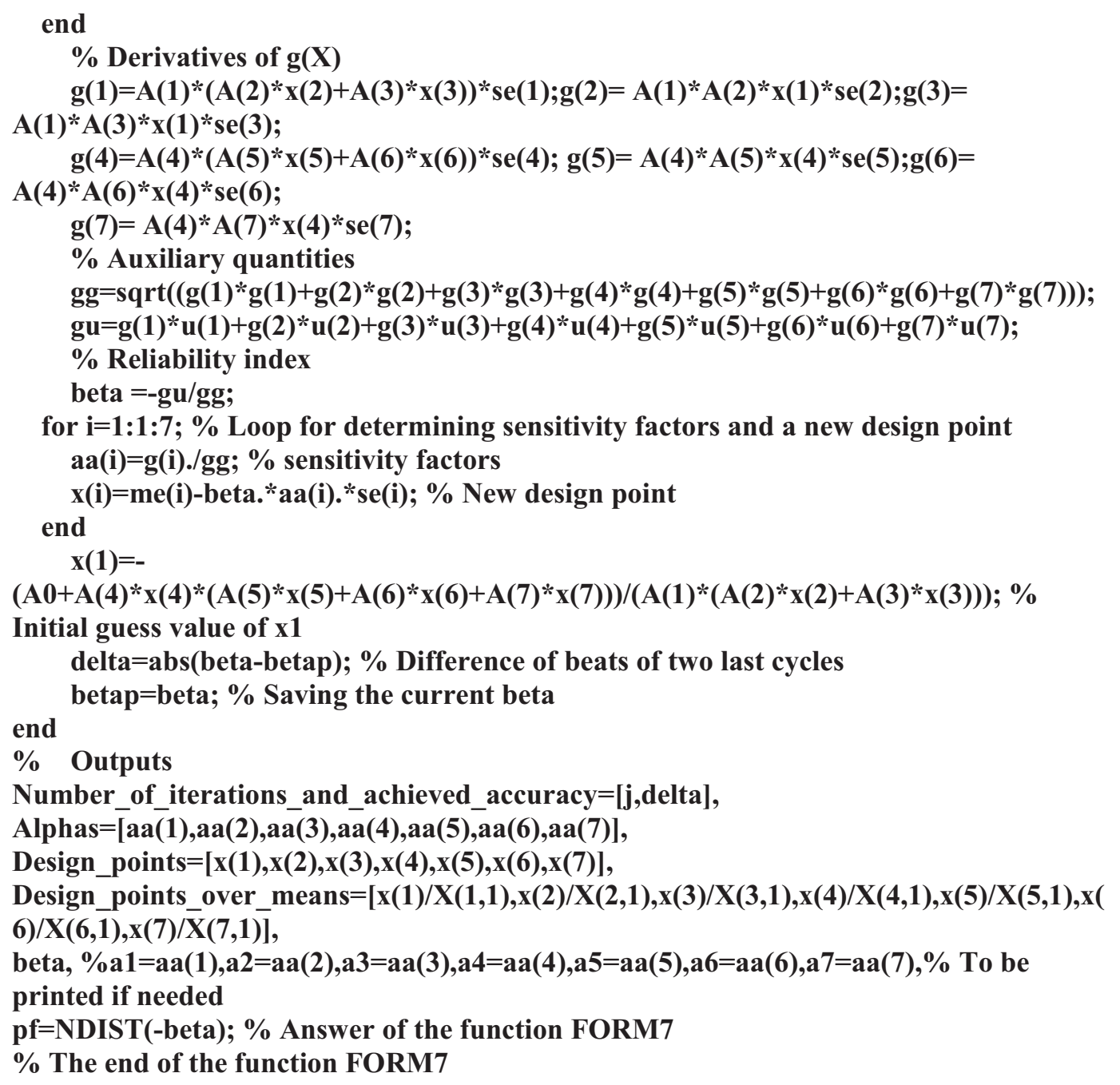




\section{ABOUT THE AUTHOR}

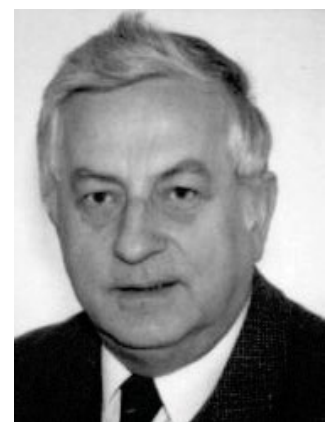

Prof. Ing. Milan Holický, Ph.D., DrSc., Klokner Institute, CTU, Prague

Professor Doctor Milan Holický obtained his civil engineering degree at the Czech Technical University in Prague, and his doctoral degree at the University of Waterloo in Canada. He is author and co-author of more than 300 scientific publications which include various textbooks and five monographs (three of which are in English, published by Elsevier and Thomas Telford Publications), and is at present actively involved in the research of structural reliability and risk assessment. He is currently taking part in international research within the Conseil International du Batiment (CIB), the Joint Committee for Structural Safety (JCSS), (particularly in WP 2 Risk Assessment), and in international standardisation within the International Organisation for Standardisation (ISO). Since 1991 he has represented the Czech Republic in the European Committee for Standardisation (Comité Européen de Normalisation or CEN) in the Technical Committee 250 (TC 250)'s "Structural Eurocodes". Since 1990 Professor Holický has cooperated closely with BRE Watford in the United Kingdom, of which he has been a BRE associate since 1997. In 1999 he assumed responsibility for the long-term research project "Reliability and Risk Assessment of Technical Systems" which receives the support of the Czech Republic's Ministry of Education. More recently, Professor Holický was a coordinator of the European project "Development of Skills Facilitating Implementation of Structural Eurocodes", which was completed over a period of three years, from 2003 to 2005, by institutions from seven partner countries. He has been employed since 1965 at the Klokner Institute of the CTU, Prague and is currently a lecturer in the Faculties of Civil Engineering and Architecture. 